\title{
Time-resolved X-ray phase-contrast tomography
}

\author{
Dissertation \\ zur Erlangung des mathematisch-naturwissenschaftlichen Doktorgrades \\ "Doctor rerum naturalium" \\ der Georg-August-Universität Göttingen \\ im Promotionsprogramm ProPhys \\ der Georg-August University School of Science (GAUSS)
}

vorgelegt von

Aike Ruhlandt

aus Eschwege

Göttingen, 2017 


\section{Betreuungsausschuss:}

Prof. Dr. Tim Salditt

Institut für Röntgenphysik,

Georg-August-Universität Göttingen

Prof. Dr. Jörg Enderlein

Drittes Physikalisches Institut,

Georg-August-Universität Göttingen

\section{Mitglieder der Prüfungskommission:}

Referent:

Prof. Dr. Tim Salditt

Institut für Röntgenphysik,

Georg-August-Universität Göttingen

1. Korreferent: Prof. Dr. Jörg Enderlein

Drittes Physikalisches Institut,

Georg-August-Universität Göttingen

Weitere Mitglieder der Prüfungskommission:

Prof. Dr. Claus Ropers

Viertes Physikalisches Institut,

Georg-August-Universität Göttingen

Dr. Alexander Egner

Laser-Laboratorium Göttingen e.V.

Prof. Dr. Sarah Köster

Institut für Röntgenphysik,

Georg-August-Universität Göttingen

Prof. Dr. Rainer Kree

Institut für Theoretische Physik,

Georg-August-Universität Göttingen

\section{Tag der mündlichen Prüfung:}

27. November 2017 




\section{Contents}

1 Introduction $\quad 9$

2 Mathematical foundations of signal processing 13

2.1 Important properties of the Fourier transform . . . . . . . . . . . . . . 13

2.1.1 Fourier filter and the Convolution Theorem . . . . . . . . . . 15

2.2 The discrete Fourier transform . . . . . . . . . . . . . . . . . . 17

2.2.1 The Sampling Theorem . . . . . . . . . . . . . . . . . . 18

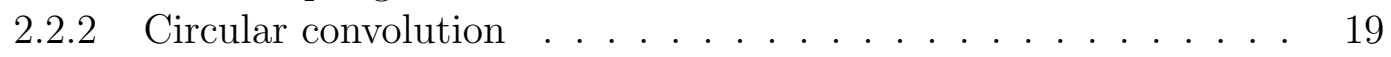

2.2.3 Interpolation in Fourier space . . . . . . . . . . . . . . . 20

3 Looking into things: Fundamental aspects of X-ray tomography 23

3.1 From the wave equation to the projection approximation . . . . . . . . 24

3.1.1 Electromagnetic waves . . . . . . . . . . . . . . . 24

3.1.2 The projection approximation . . . . . . . . . . 26

3.2 Projections from different perspectives . . . . . . . . . . . . . . . 29

3.2.1 The Fourier Slice Theorem . . . . . . . . . . . . . . . . . 29

3.2 .2 The Radon transform . . . . . . . . . . . . . . . . . . . . 30

3.3 Direct tomographic reconstruction . . . . . . . . . . . . . . . . . 32

3.3.1 Discrete sampling . . . . . . . . . . . . . . . . . . 33

3.3 .2 The backprojection . . . . . . . . . . . . . . 33

3.3.3 Filtered layergram and filtered backprojection . . . . . . . . . 35

3.4 Algebraic reconstruction techniques . . . . . . . . . . . . . . . . 39

3.4.1 Consistency conditions . . . . . . . . . . . . . . . . . 40

3.4.2 Iterative reconstruction techniques . . . . . . . . . . . . . 42

4 Wave field propagation $\quad 47$

4.1 The diffraction formula . . . . . . . . . . . . . . . . . . . . . 47

4.1.1 Fresnel-Kirchhoff theory . . . . . . . . . . . . . . . 47

4.1.2 Rayleigh-Sommerfeld theory . . . . . . . . . . . . . 50

4.1.3 Comparison of theories . . . . . . . . . . . . . . 51

4.2 Fresnel propagation . . . . . . . . . . . . . . . . . 52

4.3 Numerical propagation . . . . . . . . . . . . . . . . . 55

4.3.1 Discretising for different geometries . . . . . . . . . . . . . 55

4.3.2 Considering band limits . . . . . . . . . . . . . . . 58

4.4 3D propagation . . . . . . . . . . . . . . . . 64

4.5 The Fresnel-Scaling Theorem . . . . . . . . . . . . . 66

5 Propagation-based phase-contrast imaging $\quad 69$

5.1 Information content of a wave field . . . . . . . . . . . . . . . . 70

5.1.1 The diffraction limit . . . . . . . . . . . . . . . 71 
5.1 .2 Imaging regimes . . . . . . . . . . . . . . . . . . . . 71

5.1 .3 Contrast transfer . . . . . . . . . . . . . . . 75

5.1.4 Numerical imaging constraints . . . . . . . . . . . . . . . 78

5.2 Direct phase retrieval: Dealing with lost information . . . . . . . . 79

5.2.1 Holographic phase retrieval . . . . . . . . . . . . . 80

5.2.2 CTF-based reconstruction .............. . . 81

5.3 Iterative phase retrieval: Replacing lost information . . . . . . . . . . 83

5.4 Phase retrieval in $3 \mathrm{~d} \ldots \ldots \ldots$. . . . . . . . . . . . 86

5.4.1 Consistency as a constraint: Iterative reprojection phase-retrieval 87

5.4.2 3d propagation-based phase retrieval . . . . . . . . . . . 91

5.4.3 Performance on experimental data . . . . . . . . . . . . . 95

5.4 .4 Summary and outlook . . . . . . . . . . . . . . 96

6 Dynamic tomography of sedimenting micro-spheres $\quad 99$

6.1 Motion artefacts and positioning error . . . . . . . . . . . . . 102

6.2 The sedimenting spheres experiment . . . . . . . . . . . . . 108

$6.2 .1 \quad \mathrm{P} 10$ beamline setup . . . . . . . . . . . . . . . . . . . 108

6.2.2 X-ray waveguide illumination . . . . . . . . . . . . . . 110

6.2.3 Choice of sample . . . . . . . . . . . . . . . . . 112

6.2.4 Sample preparation and measurement . . . . . . . . . . . 114

6.3 Data analysis . . . . . . . . . . . . . . . . . 116

6.3.1 Data conditioning and alignment . . . . . . . . . 116

6.3.2 3d particle tracking . . . . . . . . . . . . . . . . . 121

6.3 .3 Collective behaviour . . . . . . . . . . . . . . 126

6.4 Summary and conclusion . . . . . . . . . . . . . . . . . 129

7 Dynamic tomography of a burning match 131

7.1 Capturing deformation and dissolving processes . . . . . . . . . . . 131

7.2 Backprojection along dynamically curved paths . . . . . . . . . . 133

7.3 The burning match experiment . . . . . . . . . . . . . . . 136

7.4 Data analysis . . . . . . . . . . . . . . . . . 137

7.4.1 Projection retrieval and alignment . . . . . . . . . . . . 137

7.4.2 Optical flow estimation . . . . . . . . . . . . . . . 139

7.4.3 3d reconstruction . . . . . . . . . . . . . . . . . . 141

7.5 Summary and outlook . . . . . . . . . . . . . . . . . . . . 142

8 Summary and outlook $\quad 145$

9 Appendix $\quad 147$

9.1 Numerical backprojection of 2 d images . . . . . . . . . . . . . . . . 147

9.2 Convolution-based numerical propagation . . . . . . . . . . . . . . 149

9.3 Properties of binary zone plates . . . . . . . . . . . . . . . 152

$\begin{array}{ll}10 \text { List of Figures } & 155\end{array}$

11 Bibliography 159

11.1 Own publications . . . . . . . . . . . . . . . . . . 170 


\section{List of Abbreviations}

\begin{tabular}{ll}
\hline $1 \mathrm{~d} / 2 \mathrm{~d} / 3 \mathrm{~d} / 4 \mathrm{~d}$ & one / two / three / four-dimensional \\
ART & Algebraic reconstruction technique \\
B & Backprojection \\
CCD & Charge-coupled device (image sensor) \\
CDI & Coherent diffraction imaging \\
CT & Computed tomography \\
CTF (aCTF / pCTF) & (amplitude / phase) Contrast transfer function \\
DFT & Discrete Fourier transform \\
ER & Error reduction algorithm \\
FBP & Filtered backprojection \\
FFT & Fast Fourier Transform \\
FOV & Field of view \\
FPS & Frames per second \\
FST & Fourier slice theorem \\
FT & Fourier transform \\
HE & Helmholtz equation \\
HIO (mHIO) & (modified)Hybrid input output algorithm \\
HLCC & Helgason-Ludwig consistency condition \\
IRP & Iterative reprojection phase-retrieval \\
IRR & Iterative reconstruction-reprojection \\
KB & Kirkpatrick-Baez mirror \\
MRT & Magnetic Resonance Tomography \\
MSD & Mean squared displacement \\
NA & Numerical Aperture \\
OAV & On axis view (microscope) \\
OF & Obliquity factor \\
PIV & Particle image velocimetry \\
PSD & Power spectral density \\
PSF & Point spread function \\
RAAR & relaxed averaged alternating reflections \\
ROI & Region of interest \\
SSD & Solid-state drive (data storage device) \\
SIRT & Simultaneous iterative reconstruction technique \\
TIE & Transport of intensity equation \\
WG & Waveguide \\
&
\end{tabular}





\section{Introduction}

Wilhelm ConRad Röntgen's discovery of X-rays, published in 1895 [1], became instantly famous around the world. For the first time, it became possible to obtain a non-destructive view of a solid object's interior, demonstrated by a radiogram of a human hand and its bone structure. This application immediately aroused the interest of the medical community and in a short time the new field of radiography developed into an indispensable tool of medical diagnosis, already playing a prominent role in the presentation speech of the first Nobel Prize in history, which was awarded to Röntgen in 1901.

However, for many applications, the shade-like X-ray projections are insufficient. Instead, knowledge of the entire three-dimensional (3d) structure is necessary. In the 1960s, scientists and engineers such as William H. Oldendorf, Allan M. CorMACK and Godfrey Hounsfield were responsible for the development of computerised tomography (CT): Advances in computer technology in particular made it possible to determine the $3 \mathrm{~d}$ structure of an object from a large number of X-ray images taken from different directions. Once again, this development was honoured with the Nobel Prize in Medicine in 1979.

Owing to the penetration power of hard X-rays, the method is uniquely suitable for the investigation of bulk structures of unsliced, unstained, and optically opaque specimen. Due to this weak interaction with matter, however, thin samples, in particular of low density and light element tissues hardly influence the X-ray beam - at least in the context of traditional radiography which is sensitive only to the amplitude (transmission) of the X-ray wave. This long-standing limitation has been overcome in revolutionary ways by the development of phase contrast methods in the last two decades [2-5]. These techniques also take the considerably stronger phase shifting properties of the objects into account, enabling the visualisation of weakly or non-absorbing samples in biomedical imaging, as well as nanoscale structures in material science. These types of samples profit from another important property of X-rays, too: Their wavelength is much smaller than that of visible light used in a conventional microscope, pushing the theoretical resolution limit to the sub-nanometre range.

As an alternative to various analyser-based methods $[3,6]$, phase contrast can be easily achieved without additional optical elements by free space propagation of (partially) coherent beams behind the object [7-9]. The development of interference patterns over the distance between sample and detector encodes the phase information into measurable brightness variations - a method called (in-line) holography, invented by Dennis Gabor and awarded with the 1971 Nobel Prize in Physics. Such scanningfree methods in combination with highly brilliant ${ }^{1}$ synchrotron sources developed over the last decades now allow for rapid image acquisition. This opens up the possibility to measure $3 \mathrm{~d}$ dynamics in addition to the $3 \mathrm{~d}$ structure, enabling a new insight into

${ }^{1}$ I.e. providing very intense, nearly monochromatic and collimated X-rays. 
a large variety of processes not only on the micro-scale. This combination of fastest phase contrast imaging with tomography is the main topic of this thesis.

A wide range of relevant applications comes to mind: In order to track down turnover rate, reaction products, or the evolving material structure in chemical reactions like heterogeneous catalysis, dynamical changes have to be determined within the entire volume, not only from projections or surface observations. In biology, too, numerous kinds of processes can be fully understood only in all four dimensions, including the complex formation of filament networks during cell division, the interplay of muscles at certain motions, or even the interaction of individuals, from reproduction to flocking behaviour. Moreover, fundamental technical applications depend on the knowledge of 3d dynamics, for example the flow of granular matter like sand suddenly stalling in a funnel.

However, the challenges involved in $4 \mathrm{~d}$ tomography are as numerous as its benefits. They include practical considerations, e.g. fast sample rotation for rapid acquisition of projections along different directions, or questions concerning fast data acquisition and processing as well as handling of large datasets. But there are also many fundamental questions such as the proper application of a priori knowledge for phase-contrast imaging or considerations regarding the best compensation of motion-related inconsistencies in tomographic reconstruction, to mention only a few. In this work, several approaches are presented to overcome these issues.

The standard procedure in evaluating phase contrast tomography data is a two-step reconstruction procedure. First, the phase retrieval is carried out, i.e. decoding the complex-valued projections from the detector's intensity measurements, separately for each projection angle. Second, all projections are combined to a three dimensional volume using tomographic reconstruction techniques. Both steps are in general ill-posed inverse problems with numerous solutions explaining the measured data. Hence, additional assumptions are required to select an appropriate result. In this context, the step of phase retrieval is considered the most challenging. As demonstrated in previous works $[10,11]$, a combination of both steps, i.e. utilising the concept of tomographic consistency as a constraint for phase retrieval, considerably improves the reconstruction quality. Here, this idea is developed further into a fast and practical iterative method based on the novel concept of virtual free-space propagation of entire 3d objects: Under certain conditions, both reconstruction steps can be inverted, allowing for the phase retrieval to be carried out entirely in $3 \mathrm{~d}$ rather than in $2 \mathrm{~d}$. This yields high-quality results without the need of additional restrictive constraints by preserving the advantages of tomographic consistency and the execution speed of the conventional scheme. Moreover, a new class of $3 \mathrm{~d}$ constraints can be utilised for phase retrieval. In this context, an accurate method for the numerical propagation of wave fields is presented, which is free of the artefacts usually affecting this operation and is easily scalable to higher dimensions.

Addressing dynamical phase-contrast tomography, an experimental examination of the $3 \mathrm{~d}$ sedimentation dynamics of $\mathrm{SiO}_{2}$ microspheres in a small capillary is presented. Sedimentation is one of the oldest methods to separate particles of different densities and shapes from each other and from fluids. However, it is still far from being completely understood even in the simplest case of monodisperse spherical particles: Long-range hydrodynamic interactions cause a complex behaviour of the spheres, including fluc- 
tuations in the sedimentation speeds up to occasional rising of particles against the direction of sedimentation. Such processes can only be fully investigated with access to the $3 \mathrm{~d}$ dynamics which are provided in great detail by the dynamical phase-contrast tomography introduced here.

Revealing the evolution of an entire $3 \mathrm{~d}$ structure in time to the fullest potential of this technique, however, can only be accomplished if recording schemes and reconstruction algorithms are generalised from the conventional static case to meet the challenges of dynamic CT. To this end, the method of "backprojection along dynamically curved paths" is presented. It is based on deriving motion approximated from optical flow analysis of direct reconstructions and feeding this information into the tomographic reconstruction geometry. Structure and dynamics are recovered from the continuous stream of projection data acquired while rapidly rotating the sample, with a considerable reduction of artefacts which typically compromise conventional reconstruction. As a proof of concept, this approach is demonstrated on a burning wooden match with a spatial and temporal resolution allowing to observe the entire structure but also small wooden segments breaking down.

This thesis is organised as follows: First, the Fourier transform as the mathematical basis of many concepts in this work is introduced, along with its most important properties, the notation and conventions for the subsequent chapters. Second, the theoretical foundations of X-ray imaging are briefly summarised, yielding a mathematical description of the so-called projection approximation, i.e. the radiograms obtained from $\mathrm{X}$-rays passing through a sample. Subsequently, the main principles of tomography are introduced, illustrating how the sophisticated combination of projections from different perspectives can reveal a $3 \mathrm{~d}$ representation of a sample. Special attention is drawn to a detailed description of the numerical implementation of these concepts.

The fourth chapter recapitulates the evolution of the projection's wave field from the sample to a distant detector with the goal of obtaining phase contrast. As before, special attention is given to the numerical issues of propagation. A method for accurate propagation of wave fields is presented, considering the discretised sampling. The section concludes with an extension of the concept of propagation to $3 \mathrm{~d}$ objects, combining propagation and tomography, which will prove to be a useful numerical tool in the following.

Based on the question to which extent information about an object can be measured at different regions of the downstream wave field, the fifth chapter is dedicated to phase contrast and phase retrieval, i.e. the reconstruction of the projection from the intensity variations on the detector. In this context, approaches for utilising tomographic consistency as a constraint are detailed and demonstrated on the first experimental datasets.

The sixth chapter presents the challenges of dynamical tomography using the example of sedimenting $\mathrm{SiO}_{2}$ micro-spheres, revealing the $3 \mathrm{~d}$ dynamics of this process in great detail. The concept of backprojection along dynamically curved paths, demonstrated on a match during combustion, is introduced and discussed in the seventh chapter.

Details about the numerical implementation of the methods can be looked up in the appendix, including extracts from the source code, among other things. 



\section{Mathematical foundations of signal processing}

This thesis touches upon topics like wave optics, image processing and analysis as well as tomographic reconstruction. Common to all of them is the Fourier transform as an indispensable mathematical tool. In this chapter, the most important concepts and properties of Fourier theory are briefly introduced, along with the mathematical notation and the conventions used throughout this work.

\subsection{Important properties of the Fourier transform}

If a function $f: \mathbb{C}^{n_{d}} \rightarrow \mathbb{C}^{n_{d}}$ satisfies the DIRICHLET conditions in an arbitrary interval, i.e. only a finite number of jump discontinuities exist (all one-sided limits exist and are finite), while $f$ is continuous and piecewise monotonic otherwise, and moreover the integral $\int_{-\infty}^{\infty}|f(\vec{r})| \mathrm{d} \vec{r}$ converges, $f$ can be uniquely expessed in the interval as a superposition of oscillations [12]

$$
f(\vec{r})=\frac{1}{(2 \pi)^{n_{d}}} \int_{-\infty}^{\infty} \hat{f}(\vec{\nu}) \exp (i \vec{\nu} \cdot \vec{r}) \mathrm{d} \vec{\nu}
$$

with $n_{d}$ the number of dimensions of $\vec{r}$. A single oscillation $\exp (i \vec{\nu} \cdot \vec{r})$ is denoted as a (frequency) mode in the following and the complex amplitude distribution function $\hat{f}$ of the modes is called the spectrum or the Fourier transform of $f$. Alternatively, $\hat{f}$ is denoted as the frequency domain, Fourier space or reciprocal space representation of the original signal. The latter term describes the famous property that the width of a function is inverted by the transformation between real space and Fourier space defined by the coordinate sets $\vec{r}$ and $\vec{\nu}$, respectively. The most drastic example is the transformation of a single oscillation infinitely extended in the frequency domain

$$
\frac{1}{(2 \pi)^{n_{d}}} \int_{-\infty}^{\infty} \exp \left(-i \vec{\nu} \cdot \vec{r}_{0}\right) \exp (i \vec{\nu} \cdot \vec{r}) \mathrm{d} \vec{\nu}=\delta_{D}\left(\vec{r}-\vec{r}_{0}\right)
$$

resulting in an infinitely sharp DIRAC $\delta$-peak, precisely localised at $\vec{r}_{0}$. This equation can be considered as the definition of the $\delta$-distribution as introduced by FouRIER and CAUCHY. If $\vec{r}$ does not exactly match $\vec{r}_{0}$, the multiplication in the integral yields a beat oscillation that is symmetrically positive and negative, cancelling in the integration. 
Only an exact match contributes to the integral with an infinite value. As can be verified easily, the inverse case

$$
\frac{1}{(2 \pi)^{n_{d}}} \int_{-\infty}^{\infty} \delta_{d}\left(\vec{\nu}-\vec{\nu}_{0}\right) \exp (i \vec{\nu} \cdot \vec{r}) \mathrm{d} \vec{\nu}=\frac{1}{(2 \pi)^{n_{d}}} \exp \left(\overrightarrow{\nu_{0}} \cdot \vec{r}\right)
$$

transforms the localised $\delta$-peak to an infinitely extended mode. This duality motivates the definition of the Fourier transform ${ }^{1} \mathcal{F}: f(\vec{r}) \in \mathbb{C}^{n_{d}} \rightarrow \hat{f}(\vec{\nu}) \in \mathbb{C}^{n_{d}}(\mathrm{FT})$ as

$$
\mathcal{F}: \hat{f}(\vec{\nu})=\int_{-\infty}^{\infty} f(\vec{r}) \exp (-i \vec{\nu} \cdot \vec{r}) \mathrm{d} \vec{r}
$$

as well as the scaling factor $1 /\left(2 \pi^{n_{d}}\right)$ in the inverse Fourier transform $\mathcal{F}^{-1}: \hat{f}(\vec{\nu}) \in$ $\mathbb{C}^{n_{d}} \rightarrow f(\vec{r}) \in \mathbb{C}^{n_{d}}$ already introduced in Eq. (2.1). This factor, ensuring that $f=\mathcal{F}^{-1}[\mathcal{F}(f)]$, is often equally distributed between the forward and backward transformations. Here, however, in order to improve the legibility of most subsequent equations, only the backtransform is normalised, as is customary in discrete FT and signal processing. We therefore have to consider that according to PLANCHEREL's formula, the signal power of the FT changes during the transformation:

$$
\int_{-\infty}^{\infty}|f(\vec{r})|^{2} \mathrm{~d} \vec{r}=\frac{1}{(2 \pi)^{n}} \int_{-\infty}^{\infty}|\hat{f}(\vec{\nu})|^{2} \mathrm{~d} \vec{\nu}
$$

Next to linearity $\mathcal{F}[a \cdot f(\vec{r})+b \cdot g(\vec{r})]=a \mathcal{F}[f(\vec{r})]+b \mathcal{F}[g(\vec{r})]$, other important properties of the FT for this work are summarised in the following table.

\begin{tabular}{rccl}
\hline Operation / property & Signal & Fourier transform & Comment \\
\hline scaling with $a \in \mathbb{R}$ & $f(a \vec{r})$ & $\frac{1}{|a|} \hat{f}(\vec{\nu} / a)$ & inv. frequency scaling \\
shifting by $\vec{a} \in \mathbb{R}^{n_{d}}$ & $f(\vec{r}-\vec{a})$ & $\exp (i \vec{a} \cdot \vec{\nu}) \hat{f}(\vec{\nu})$ & mult. with oscillation \\
$n$-th derivative along $x$ & $\frac{\partial^{n}}{\partial x^{n}} f(\vec{r})$ & $\left(i \nu_{x}\right)^{n} \hat{f}(\vec{\nu})$ & multiplication with $\nu_{x}^{n}$ \\
complex conjugation & $f^{*}(\vec{r})$ & $\hat{f}^{*}(-\vec{\nu})$ & conj. and point reflection \\
real function & $f(\vec{r}) \in \mathbb{R}$ & $\hat{f}(\vec{\nu})=\hat{f}^{*}(-\vec{\nu})$ & Hermitian function \\
\hline
\end{tabular}

The Fourier transforms for $n_{d}=1$ of special functions and distributions used in this work are summarised in the next table:

\footnotetext{
${ }^{1}$ Note that both the mathematical transformation $\mathcal{F}$ as well as the result $\hat{f}$ are denoted as a Fourier transform.
} 


\begin{tabular}{rrr}
\hline Description & Signal & Fourier transform \\
\hline Gaussian function & $\exp \left(-a x^{2}\right)$ & $\sqrt{\pi / a} \exp \left(-\nu_{x}^{2} / 4 a\right)$ \\
Heaviside step function $\Theta(x)$ & $\Theta(x):=(1+\operatorname{sgn}(x)) / 2$ & $\pi \delta_{D}\left(\nu_{x}\right)+i / \nu_{x}$ \\
window function $\Pi(x)$ & $\Pi(x):=\Theta(x+1 / 2)-\Theta(x-1 / 2)$ & $\operatorname{sinc}\left(\nu_{x} / 2\right)$ \\
modulus & $|x|$ & $-2 / \nu_{x}^{2}$ \\
\hline
\end{tabular}

\subsubsection{Fourier filter and the Convolution Theorem}

Revealing the spectrum of a signal, the FT offers the possibility to filter a function by manipulating specific frequency components. Well-known applications are e.g. a lowpass filter damping high-frequency noise from audio signals, a high-pass filter removing low spatial-frequency background variations in images or a bandpass filter manipulating the values in a specific frequency interval. Such filters can simply be applied by multiplying the signal's Fourier transform point-wise with an appropriate weighting function, followed by a backtransform. In this way many other operations can be carried out, notably a shift or a derivation as shown in the above table. As a convention, we denote all such operations carried out by a multiplication of two functions in Fourier space as filter operations.

According to the convolution theorem, all filter operations with the weighting function being Fourier transformable can be equivalently expressed in real space by

$$
\mathcal{F}^{-1}[\hat{f}(\vec{\nu}) \cdot \hat{g}(\vec{\nu})]=(f * g)(\vec{r}),
$$

where the convolution of both functions $f, g$ is defined as

$$
(f * g)(\vec{r}):=\int_{-\infty}^{\infty} f(\vec{\tau}) g(\vec{r}-\vec{\tau}) \mathrm{d} \vec{\tau}=\int_{-\infty}^{\infty} f(\vec{r}-\vec{\tau}) g(\vec{\tau}) \mathrm{d} \vec{\tau} .
$$

The functions are shifted relative to each other and the overlapping volume is calculated for each shift. This is similar to the idea of a filter, i.e. one of the functions $(\simeq$ the signal) gets changed by the so-called convolution kernel ( $\simeq$ filter function). A good example is smoothing a function by convolution with a Gaussian kernel. In many cases, however, convolution is considered from another point of view: Consider e.g. the convolution of a distribution of $\delta$-peaks with an image, as exemplified in Fig. 2.1. The image gets reproduced at each peak's position, weighted with the pre-factor of the $\delta$-peak. In this case, the convolution produces a repetition and shift - usually not associated with a filter. This can be used as a mathematical description of objects with many identical features, e.g. a distribution of similar spheres in solution or a crystal where the unit cell is convolved with the crystal lattice. Of course, like the equivalent filter, a convolution finds numerous applications, including the description of entire optical systems by convolving the ideal image of a sample with the so-called pointspread-function (PSF) of the optical system, i.e. the image obtained by excitation with 
a $\delta$-peak-like signal. Note that the inverse operation, a deconvolution which obtains the original signal from the given convolution and kernel is often not possible. It requires a division by the corresponding filter in Fourier space, often impossible due to zeros in the filter or extreme enhancement of noise in practical applications.
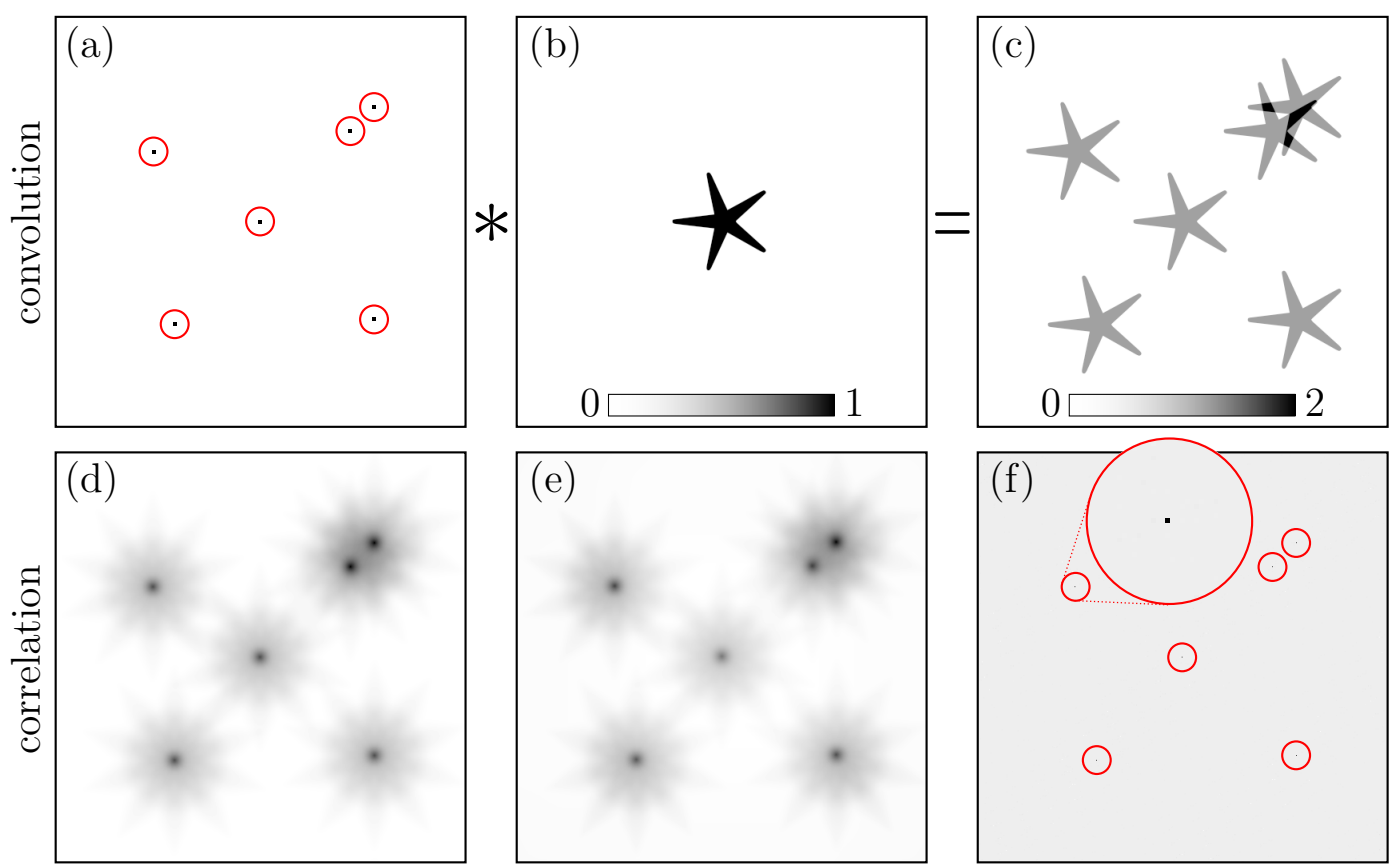

Figure 2.1: Convolution and correlation. The convolution of the "signal" (a) with the "kernel" (b) yields a superposition of shifted kernels (c). For better visibility, the delta peaks have been widened and surrounded by a circle in the picture. The original positions (a) can be retrieved approximately by a correlation of (b) and (c). The result of a cross-correlation is shown in (d), a normalised cross-correlation, very similar in this case, is depicted in (e) while (f) shows the result obtained by a phase correlation. Since the peaks in (f) are very small, they are highlighted by circles, too.

Similar to the convolution, but often used for the inverse purpose is the (cross) correlation of two functions $f, g$, defined as

$$
(f \star g)(\vec{r}):=\left[f^{*}(-\vec{\tau}) * g(\vec{\tau})\right](\vec{r})=\int_{-\infty}^{\infty} f^{*}(\vec{\tau}) g(\vec{\tau}+\vec{r}) \mathrm{d} \vec{\tau} .
$$

Notice the star $\star$ instead of the asterisk $*$ for the convolution, while the superscript asterisk $f^{*}$ denotes the complex conjugate. When shifting the two functions relative to each other, the result of the correlation will be maximum when the functions are most similar, i.e. the maxima meet for best enhancement and the minima match for minimum damping of each other. With this property, the correlation is commonly used for image registration but also to retrieve the positions from similar superposed features, as demonstrated in Fig. 2.1 for the data initially obtained by a convolution. 
Practical formulations robust against noise are e.g. the normalised cross-correlation as defined in [13] and the so-called phase correlation $P C$, given by

$$
P C:=\mathcal{F}^{-1}\left[\frac{\mathcal{F}(f) \cdot \mathcal{F}(g)^{*}}{\left|\mathcal{F}(f) \cdot \mathcal{F}(g)^{*}\right|}\right]
$$

Here, the nature of convolution and correlation can be well observed: Consider $g$ as a shifted version of $f$. In that case, the only difference in Fourier space is that $g$ is multiplied by a 'shifting' oscillation. By normalising, only this wave remains and the backtransfom results in a $\delta$-peak shifted about the same distance from the origin as both functions are shifted with respect to each other.

\subsection{The discrete Fourier transform}

Data recording and processing is mostly carried out digitally. Here, a function $f$ is represented by discretised values $f_{j}$ at a finite number of sampling points $x_{j}, j \in \mathbb{Z}$, usually arranged on a Cartesian grid. In two dimensions (2d), the quadratic arrangement of sample points is commonly referred to as picture elements (pixels) and as cubic volume elements (voxels) in $3 \mathrm{~d}$. For convenience, let us assume an $n_{d}=1$ dimensional (1d) interval divided by an even number $N$ of equidistant sampling points separated by $\Delta$. The inverse $1 / \Delta$ is usually referred to as the sampling rate. From the $N$ sampled points, only $N$ independent output values of a discretised Fourier transform (DFT) can be expected. Hence, the DFT represents the Fourier transform $\hat{f}$ as a superposition of $N$ harmonic modes $\exp (-i j k 2 \pi / N)$ with $k=-N / 2 \ldots N / 2 \in \mathbb{Z}[14]$ :

$$
\hat{f}_{k}=\sum_{j=0}^{N-1} f_{j} \exp (-i j k 2 \pi / N)
$$

with the inverse transform

$$
f_{j}=\frac{1}{N} \sum_{k=0}^{N-1} \hat{f}_{k} \exp (i j k 2 \pi / N) .
$$

Note that in the latter case the summation could be changed from 0 to $N-1$ because the function is periodic in both $j$ and $k$ with period $N$. The multidimensional DFT can be expressed by an arbitrary consecutive execution of $1 \mathrm{~d}$ DFTs along each dimension. The runtime of the DFT, as defined in Eq. (2.10), is proportional to $\mathcal{O}\left(N^{2}\right)$. However, it can be rearranged to the extremely efficient Fast Fourier Transform (FFT) with a runtime

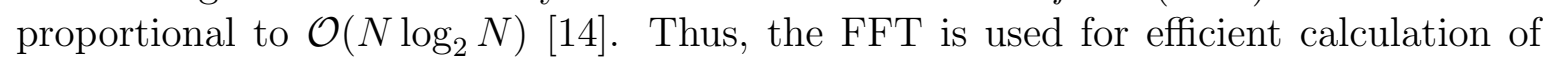
many operations such as discrete convolutions. 


\subsubsection{The Sampling Theorem}

The most important aspect of discretising a function is to which extent it is captured by the discrete sampling points and which information is lost. An interesting observation was made by SHANNON [15]: If the spectrum $\hat{f}$ of the original function $f$ is (band)limited, i.e. no frequencies $\nu$ larger than $|\nu| \leq 2 \pi L$ occur,

$$
\begin{aligned}
f(x) & =\frac{1}{2 \pi} \int_{-\infty}^{\infty} \hat{f}(\nu) \exp (i \nu x) \mathrm{d} \nu \\
& =\frac{1}{2 \pi} \int_{-2 \pi L}^{2 \pi L} \hat{f}(\nu) \exp (i \nu x) \mathrm{d} \nu
\end{aligned}
$$

the function can be reconstructed completely from discrete sampling points, provided their distance is equal to or smaller than $1 / 2 L$ :

$$
f_{j}:=f\left(\frac{j}{2 L}\right)=\frac{1}{2 \pi} \int_{-2 \pi L}^{2 \pi L} \hat{f}(\nu) \exp \left(i \nu \frac{j}{2 L}\right) \mathrm{d} \nu .
$$

At this so-called NyQUIST sampling rate, the sampling points $f_{j}$ on the left side correspond to the coefficients of the Fourier-series expansion of $\hat{f}(\nu)$ as given by the right side integral with the interval $[-L: L]$ as fundamental period. Since this expansion uniquely determines $\hat{f}$ in this interval, and we demanded $\hat{f}$ to be zero outside of the interval, the sampling points $f_{j}$ completely determine the spectrum of $f$ - which in turn means that they allow to exactly reconstruct $f(x)$. Due to the duality of the FT, this also applies to the inverse case: a spatially limited function can be completely reconstructed from a sufficiently sampled discrete set of frequencies $\nu_{j}$. In [15], SHAnnon also specifies a reconstruction formula which is known as the WHITTAKER-SHANNON interpolation formula:

$$
f(x)=\sum_{j=-\infty}^{\infty} f_{j} \cdot \operatorname{sinc}[\pi(2 L x-j)] .
$$

Each sampling point is replaced by a sinc-function defined as $\operatorname{sinc}(x):=\sin (x) / x$ with the maximum at the point's position and with zeros at the positions of all other sampling points. Clearly, in practice the sum cannot be infinite but can still represent the function within an interval in good approximation. We will look at this interpolation in more detail below. Note that these results have been generalised to multidimensional sampling on a discrete lattice of points in the PETERSEN-MiddLeton theorem [16]. Notably, many important functions are not band-limited and thus cannot be represented even by an infinite number of discrete sampling points. A famous example is the Heaviside step function $\Theta(x)$. A reconstruction from discrete sampling points will show so-called ringing artefacts, i.e. an overshooting particularly at the discontinuity that is typical for non-adequate sampling. 


\subsubsection{Circular convolution}

According to the inverse DFT formula in Eq. (2.11), the reconstruction of a signal from a set of Fourier coefficients $\hat{f}_{k}$ is not limited to the interval of the fundamental period, but periodically repeated with period $N$. This is not visible in a direct inverse DFT evaluated only within one of those periods, but is becomes significant during several operations. An important example is a discrete convolution carried out according to Eq. (2.6) by a multiplication in Fourier space. In real space, a convolution can be interpreted as the integral over the product of two functions with respect to their relative shift. If these functions are only known on a certain interval, they are usually interpreted as being compactly supported, i.e. zero everywhere outside the respective interval. Thus, with increasing relative shift, more and more of the integrand turns zero. However, when the DFT is used to calculate the convolution, the function values that leave the interval on the right side when increasing the relative shift re-enter the interval from the left, as sketched in Fig. 2.2. Because of this behaviour, this type of discrete convolution is called a circular convolution. In order to obtain the same results as for the compactly supported functions in real space, both functions have to be padded with zeros to an interval twice the size, and the resulting convolution has to be cut to the original interval size. The performance gain from using the FFT is still tremendous despite these additional steps.

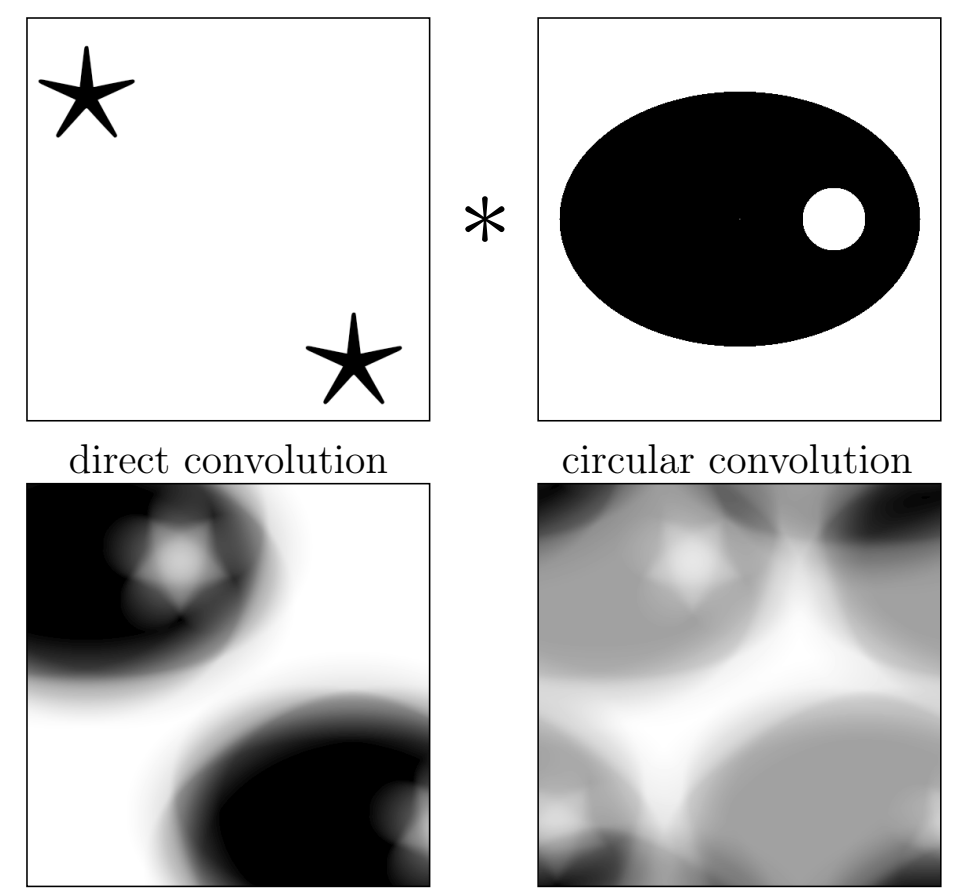

Figure 2.2: Usually, images are assumed to be zero-padded in a discrete convolution, leading to the result on the left side. However, when the convolution is implemented via the FFT according to the convolution theorem, a so-called circular convolution is performed, periodically repeating the input images. Explicit zero padding to twice the original size is required to obtain the same results as in a direct convolution. 


\subsubsection{Interpolation in Fourier space}

Numerous operations on the discretised grid of sampling points require an interpolation, i.e. the estimation or calculation of function values between the sampling points from the surrounding known values. In this work, the term mostly refers to $2 \mathrm{~d}$ resampling, meaning the challenge of how to distribute the values of a pixel grid $A$ to the pixels of grid $B$, which may be shifted, rotated, scaled or even arbitrarily deformed with respect to $A$. While the exact position of each transformed point can be calculated easily, the distribution of the sample values is less straightforward and many methods exist for this purpose, from

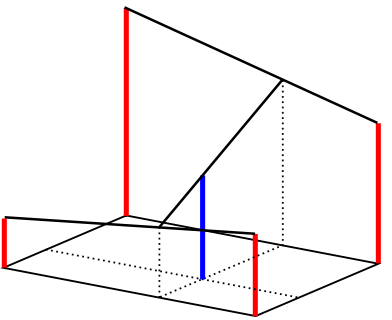

Figure 2.3: Principle of bilinear interpolation. See text for details. simple nearest-neighbour attribution to high-order polynominal fitting. Many implementations rely on the well-known bilinear interpolation because it is free from overshooting function values as known from higher order interpolation, which can yield negative values from strictly positive input causing problems in further steps of data processing, for example. A new pixel value is obtained from the four adjacent source pixels as sketched in Fig. 2.3: Each of two straight lines connects two points along the first coordinate. These two lines are then connected by a third straight line that is parallel to the second coordinate axis and runs through the position of the pixel. The value of this third line at the position of the new pixel gives the interpolated value of the new pixel. With same-sized pixels in $A$ and $B$, this corresponds to a weighting with the covered common pixel area.

Although they deliver good results in real space, the interpolation methods mentioned above are not suitable for an application in the Fourier space. This is illustrated in Fig. 2.4 on the example of a rotation with respect to the origin, an operation which is in theory equivalent in real and Fourier space. The interpolation step on the pixel grid results in clear differences: While the bilinear interpolation in real space yields a result as expected, the same interpolation carried out in $2 \mathrm{~d}$ Fourier space results in severe artefacts which manifest as a kind of repetition of the object in the backtransform to real space.

To better understand this effect, we consider the most basic transformation of a single $2 \mathrm{~d}$ mode $\exp \left(i\left(\nu_{x} n_{x} 2 \pi / N_{x}+\nu_{y} n_{y} 2 \pi / N_{y}\right)\right)$ for $\nu_{x} \in\left[-N_{x} / 2, N_{x} / 2\right], \nu_{y} \in\left[-M_{y} / 2, M_{y} / 2\right]$. As long as the oscillation is harmonic in the interval $\left(n_{x}, n_{y} \in \mathbb{Z}\right)$, it can be represented by a single complex pixel value in Fourier space. However, after a rotation (or one of many other operations), almost all modes are non-harmonic, i.e. their theoretical peak lies between the grid points. How can such a mode be represented in discrete Fourier space? The common approach of (bilinear) interpolation depicted in Fig. 2.5 (a), which distributes the peak over four pixels, yields a clearly wrong result in real space that vanishes at the edges of the interval (see Fig. 2.5 (b)). On the other hand, the desired mode can easily be drawn in real space (c) and its FT in Fig. 2.5 (d), with decreasing streaks that spread along the coordinate axes, hints at the solution: The 

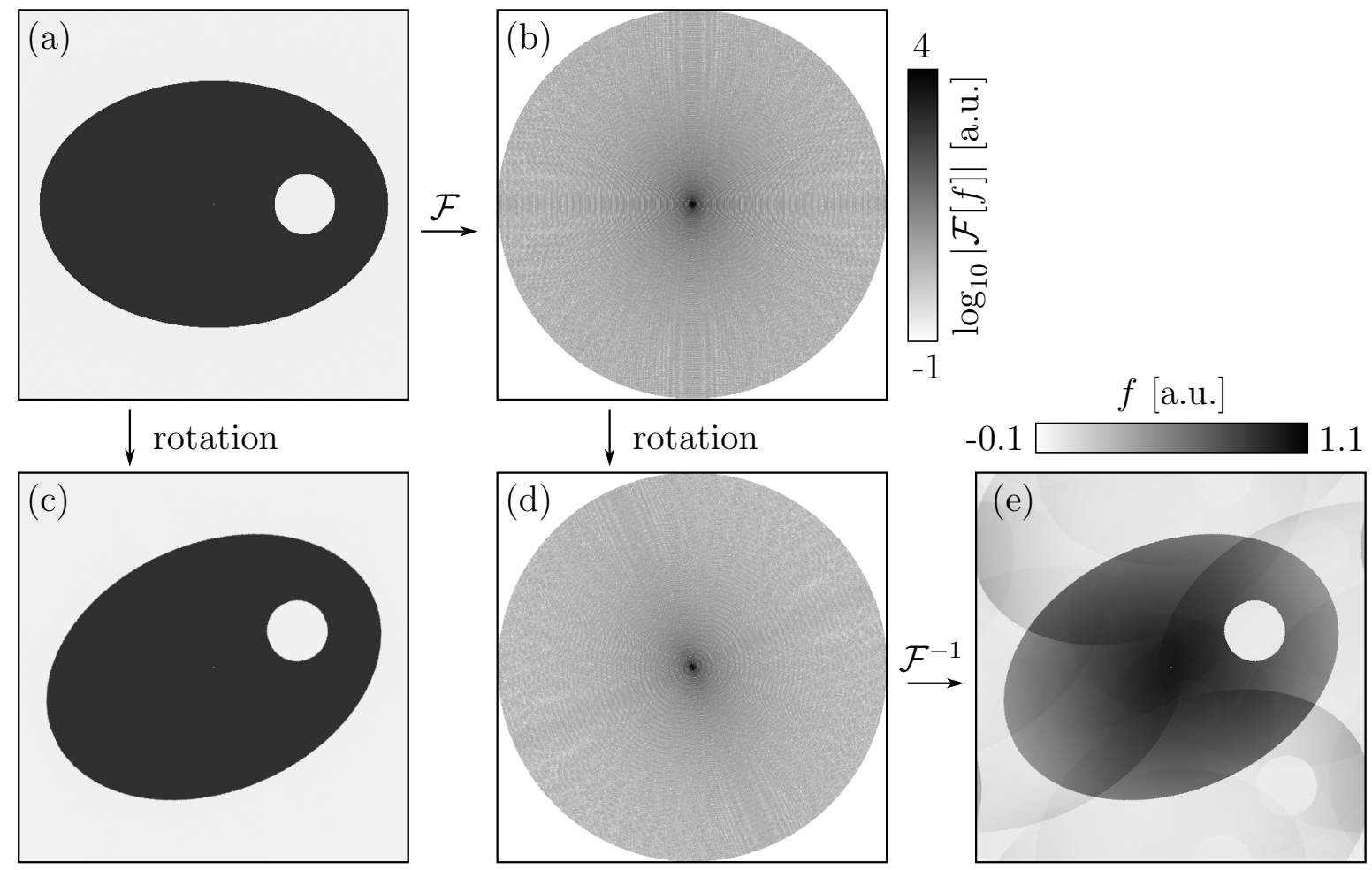

Figure 2.4: Interpolation in real- and Fourier space. The real image (a) has a radially limited spectrum (b). A rotation of (a) with bilinear interpolation in real space yields (c). The same operation carried out in Fourier space (d) results in the clearly distorted image depicted in (e). The pixel size of all images is $513 \times 513$, with the centre of the FT on a single pixel. The vertical colour bar applies to both Fourier transforms, the horizontal colour bar to all real-space images.

limited interval - resulting in a discontinuous periodic repetition of the mode - has to be taken into account. The analytical FT of such a limited mode is given by

$$
\begin{aligned}
& \int_{-N / 2}^{N / 2} \int_{-M / 2}^{M / 2} \exp \left(i\left(p_{x} x+p_{y} y\right)\right) \exp (-i \vec{\nu} \cdot \vec{r}) \mathrm{d} y \mathrm{~d} x \\
= & \mathcal{F}\left[\exp \left(i\left(p_{x} x+p_{y} y\right)\right) \cdot \Pi(x / N) \Pi(y / M)\right] \\
= & \delta_{D}\left(\nu_{x}-p_{x}, \nu_{y}-p_{y}\right) *\left[N \operatorname{sinc}\left(\nu_{x} \cdot N / 2\right) * M \operatorname{sinc}\left(\nu_{y} \cdot M / 2\right)\right] .
\end{aligned}
$$

Instead of a single $\delta$-peak, the FT is represented by the original $\delta$-pulse, convolved with a sinc-function for each dimension, which is one at the origin and zero at $\nu_{x}=j \cdot 2 \pi / N$ as well as $\nu_{y}=m \cdot 2 \pi / M$ for $j, m \in \mathbb{Z} \backslash 0$, the positions of all other points of the Cartesian grid. Thus, for a harmonic mode with the $\delta$-peak centred on a pixel, the sinc is invisible, but as soon as the spatial frequency of the mode is off-grid, the sinc becomes visible as a $1 / r$ decline in discrete sampling. Clearly, this is the Whittaker-Shannon kernel introduced above as the ideal interpolation kernel in discretised real space for a band-limited Fourier space. Here, it is applied in Fourier space for a spatially limited real space and indeed leads to the expected results [17]. However, the convolution with 
the sinc in Fourier space is a slow operation. Therefore, it is advantageous to avoid interpolations in Fourier space and to carry out the corresponding operation in real space, if possible.
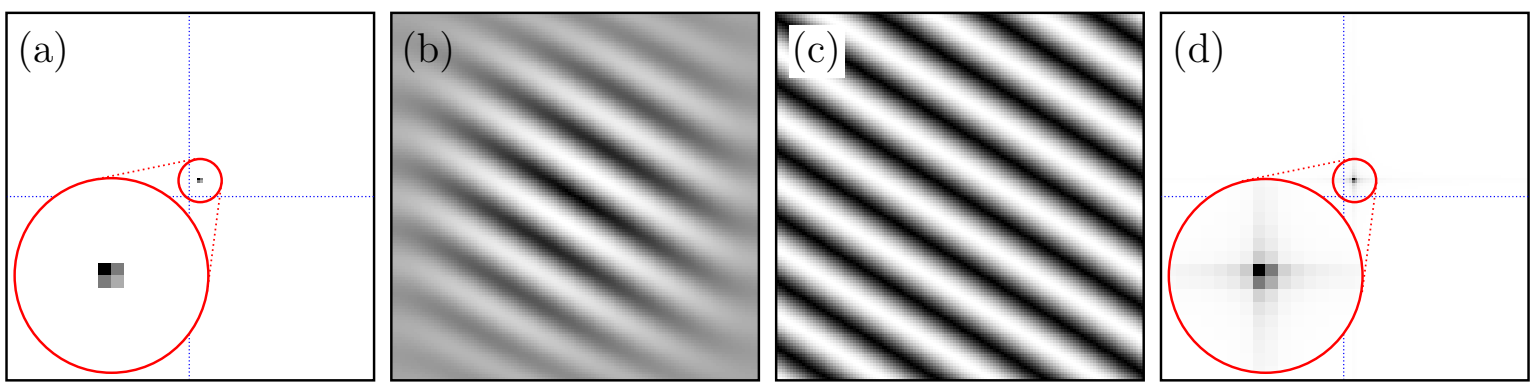

Figure 2.5: The attempt to create a non-harmonic mode in real space by a bilinear interpolation of a peak in Fourier space (a) yields a clearly wrong result (b). Contrarily, the mode defined in real space (c) Fourier transformed shows additional streaks along the coordinate axes (d), revealing that each limited mode is represented by a sinc function in the complementary space. 


\section{Looking into things: Fundamental aspects of X-ray tomography}

Despite the title of RÖNTGEN's first ground-breaking article about X-rays, they are not "A new kind of radiation" [1], but simply highly energetic electromagnetic waves. Their photon energy ranges from $100 \mathrm{eV}$ to several hundred $\mathrm{keV}$, corresponding to wavelengths between $10 \mathrm{~nm}$ and a few pm, which is the scale of molecular structures and atoms. However, the short wavelength results in a different behaviour compared to "classical" radiation such as light or radio waves. RÖNTGEN found X-rays to be only weakly absorbed by matter, possessing the ability to transmit even through large objects and "look" inside them. This immediately caught the attention of the medical community, promising revolutionary non-destructive diagnostic methods [18]. But it was precisely this advantageous weak interaction between matter and X-rays that made it impossible for RÖNTGEN to establish and characterise the radiation in terms of the properties of electromagnetic waves: Effects such as diffraction, refraction, or reflection of X-rays were simply too small. As a consequence, the manipulation of this radiation by optical elements has proven to be a tremendous challenge for experimenters to this day. Notwithstanding these special properties, many of the characteristics of X-rays and their interaction with matter can be described by classical electrodynamics.

In the next two sections we will follow this theory to derive the so-called "projection approximation" describing the images obtained when transmitting X-rays through objects. These projections then serve as the basis of X-ray tomography, the main topic of this chapter. Derived from the Ancient Greek words $\tau o ́ \mu \sigma \sigma$ (tomos) for "slice" and $\gamma \rho \alpha ́ \varphi \epsilon \iota \nu$ (graphein) meaning "to write", this method allows to obtain sections or even the entire three dimensional structure of a sample from projections along different directions. Since in practice this task can only be performed by a computer, special attention is drawn to discretisation and its numerical implementation throughout this chapter.

Historically, tomography is connected with the name of JoHAnn RADOn, who developed its mathematical foundations at the beginning of the 20th century [19]. Unaware of these findings, Allan M. Cormack developed the mathematics for X-ray based computerized tomography $(\mathrm{CT})$ and published early tomographic reconstructions from real data in 1963 [20, 21]. The first commercial CT brain scanner was developed by Godfrey Hounsfield, first demonstrated on a patient on 1st October 1971. Only eight years later, about one thousand CT scanners had been installed in hospitals, proving the large impact of this technique in clinical diagnosis. In the same year, Hounsfield and Cormack received the Nobel Prize in Medicine ${ }^{1}$ [24]. The field

\footnotetext{
${ }^{1}$ As often, not all contributions could be honoured this way. Already in 1961, the neurologist W. H. OLDENDORF published the first paper about X-ray tomography and received the earliest patent in 1963. In 1966, he published the first image of a slice through a body obtained by a self-constructed device. His invention that "would do nothing but make a radiographic cross section of a head"
} 
has been growing ever since. Today tomographic methods are used for a wide range of probes in numerous applications, ranging from magnetic resonance tomography (MRT) over seismic- and muon- to electrical impedance tomography. The range of literature is correspondingly comprehensive. For the brief introduction given in this chapter, the textbooks [25-27] have been used predominantly. The wave-optical part follows the derivations in $[3,28,29]$.

\subsection{From the wave equation to the projection approximation}

\subsubsection{Electromagnetic waves}

Classical electrodynamics can be completely described in terms of the LoRENTz force and the four MAXWELL equations. In SI units, which are used throughout this work, the latter are given by the differential equations

$$
\begin{array}{ll}
\vec{\nabla} \cdot \vec{E}=\frac{\varrho}{\varepsilon_{0}}, & \vec{\nabla} \times \vec{E}=-\frac{\partial \vec{B}}{\partial t}, \\
\vec{\nabla} \cdot \vec{B}=0, & \vec{\nabla} \times \vec{B}=\mu_{0}\left(\vec{j}+\varepsilon_{0} \frac{\partial \vec{E}}{\partial t}\right) .
\end{array}
$$

Here, $\vec{E}(\vec{r}, t)$ represents the electric field at the point $\vec{r}=(x, y, z) \in \mathbb{R}^{3}$ in threedimensional Cartesian space and at time $t \in \mathbb{R} ; \vec{B}(\vec{r}, t)$ symbolizes the magnetic field. $\vec{\nabla}=\vec{e}_{x} \partial / \partial x+\vec{e}_{y} \partial / \partial y+\vec{e}_{z} \partial / \partial z$ is the del operator, $\varrho(\vec{r}, t)$ describes the charge density and $\vec{j}(\vec{r}, t)$ the current density. The electric and magnetic field constants are given by $\varepsilon_{0}$ and $\mu_{0}$, respectively. The first equation is GAUss' law, connecting the electric field to the distribution of charges, while the non-existence of magnetic monopoles is expressed by the second equation. The observation that a varying magnetic field induces an electric field is formulated in FARADAY's law of induction and the last equation is AMPÈRE's circuital law with Maxwell's modification [3].

When applying the curl operator on both sides of the law of induction in the absence of charges $(\vec{j}=0$ and $\varrho=0)$, the linearity of the equations allows to derive the free-space wave equation

$$
\left(\frac{\partial^{2}}{\partial t^{2}}-c_{0}^{2} \vec{\nabla}^{2}\right) \vec{E}(\vec{r}, t)=0 .
$$

The field constants have been summarized as $c_{0}:=1 / \sqrt{\mu_{0} \varepsilon_{0}}$, the speed of light in vacuum. Similarly, the same partial differential equation can be obtained for the magnetic field $\vec{B}(\vec{r}, t)$. Hence, all three components of each vector field have to obey the scalar wave equation

$$
\left(\frac{\partial^{2}}{\partial t^{2}}-c_{0}^{2} \vec{\nabla}^{2}\right) \Psi(\vec{r}, t)=0
$$

(from a declining letter from a company [22]) was ignored by the industry. Even earlier, the Russian scientist TETAL'BAUM had published a work about the mathematical foundations of CT, wich "remained unknown to the English-speaking world until the early 1980s" [23]. 
Apart from vector characteristics such as polarisation, this single equation is sufficient to describe electromagnetic waves in free space if we allow $\Psi(\vec{r}, t)$ to be complex [3]. However, for the vector fields $\vec{E}$ and $\vec{B}$, only the real part of $\Psi$ has a physical meaning. Important elementary solutions of equation (3.2) are harmonic plane waves

$$
\Psi(\vec{r}, t)=A_{0} \exp \left(i \varphi_{0}\right) \exp (i[\vec{k} \cdot \vec{r}-\omega t]) .
$$

Amplitude $A_{0} \in \mathbb{R}$ and phase shift $\varphi_{0} \in \mathbb{R}$ are often combined in a complex-valued amplitude. The wave vector $\vec{k}$ is oriented in the direction of propagation, perpendicular to the wave-fronts with constant phase. Its length $k:=|\vec{k}|=2 \pi / \lambda$ is inversely proportional to the wavelength $\lambda$ and linked to the angular frequency $\omega$ by $k=\omega / c_{0}$. Another important solution is the spherical wave

$$
\Psi(\vec{r}, t)=A_{0} \exp \left(i \varphi_{0}\right) \frac{\exp (i|\vec{k}||\vec{r}|-\omega t)}{|\vec{r}|} \hat{e}_{r}
$$

Here, $\hat{e}_{r}$ is a unit vector pointing radially away from the origin of the wave at $\vec{r}=0$. Both solutions cannot exist in the real world - their infinite extent would require an infinite amount of energy - but the linearity of the wave equation allows to express more general solutions in terms of such simple functions. Following [3], this motivates a more general ansatz for equation (3.2), given by the half Fourier integral

$$
\Psi(\vec{r}, t)=\frac{1}{\sqrt{2 \pi}} \int_{0}^{\infty} \Psi_{\omega}(\vec{r}) \exp (-i \omega t) \mathrm{d} \omega,
$$

expressing a general wave field as a temporal superposition of monochromatic harmonic plane waves. Inserted into the wave equation (3.2), one obtains

$$
\frac{1}{\sqrt{2 \pi}} \int_{0}^{\infty} \underbrace{\left(\omega^{2}+c_{0}^{2} \vec{\nabla}^{2}\right) \Psi_{\omega}(\vec{r})}_{!=} \underbrace{\exp (-i \omega t)}_{\neq 0} \mathrm{~d} \omega=0 .
$$

Since the exponential function cannot be zero but the equation has to hold for all $t \in \mathbb{R}$, the first part

$$
\left[k^{2}+\vec{\nabla}^{2}\right] \Psi_{\omega}(\vec{r})=0
$$

of the integrand has to vanish everywhere. This result is known as the Helmholtz equation, describing the shape of static monochromatic waves in free space. Using equation (3.5), solutions to this partial differential equation can be superposed to general polychromatic wave fields. 


\subsubsection{The projection approximation}

In the presence of matter, highly complex interactions between X-rays and (mainly) the electrons occur. Microscopic processes like absorption, inelastic (COMPTON) and elastic (THOMSON) scattering lead to macroscopic phenomena such as refraction and (total) reflection [30]. In classical wave-optics, these essentially quantum mechanical interactions are summarised within a few macroscopic observables. Even though Xrays are able to 'sense' the discrete atomic nature of matter in many cases, the classical theorems can be applied with remarkable accuracy.

In a linear material, GAUSS' and AMPÈRE's law have to be modified such that $\vec{E}$ is complemented by the relative electric permittivity $\varepsilon_{r}$, which expresses the electric polarisability of the material. In AMPÈRE's law, $\vec{B}$ is supplemented by the inverse relative magnetic permeability $\mu_{r}$, describing the degree of magnetisation. Both dimensionless parametres $\varepsilon_{r}$ and $\mu_{r}$ are generally tensors, however, in an isotropic material they reduce to scalars.

$$
\begin{aligned}
\vec{\nabla} \cdot \varepsilon_{0} \varepsilon_{r} \vec{E} & =\varrho_{f} \\
\vec{\nabla} \times \frac{\vec{B}}{\mu_{0} \mu_{r}} & =\left(\vec{j}_{f}+\frac{\partial}{\partial t} \varepsilon_{0} \varepsilon_{r} \vec{E}\right)
\end{aligned}
$$

In a dielectric material, $\varrho_{f}=0$ and $\vec{j}_{f}=0$ vanish and the assumption of a static material allows for $\varepsilon_{r}$ and $\mu_{r}$ to be independent of time. If the magnetisation can be neglected $\left(\mu_{r}=1\right)$ and the properties of the bulk atoms vary slowly ${ }^{2}$ over length scales comparable to the wavelength $\lambda$, the MAXWELL equations can be considered as uncoupled. Then, a similar derivation as above leads to the Helmholz equation in matter [3]

$$
\left[\vec{\nabla}^{2}+k^{2} n_{\omega}^{2}(\vec{r})\right] \Psi_{\omega}(\vec{r})=0 .
$$

Here, the frequency-dependent index of refraction $n_{\omega}=\left(\sqrt{\varepsilon_{r} \mu_{r}}\right)_{\omega}$ summarizes all remaining influence of the matter on the wave. For X-rays, $n_{\omega}$ is slightly smaller than unity and often expressed in the complex form

$$
n_{\omega}=1-\delta_{\omega}+i \beta_{\omega}
$$

The positive value $\delta_{\omega} \in \mathbb{R}^{+}$describes the phase shift, $\beta_{\omega} \in \mathbb{R}^{+}$is the positive absorption decrement. As a consequence, the wave's phase velocity in matter gets larger than in empty space. However, the group velocity determining the signal propagation is still limited by the speed of light. As an example, Fig. 3.1 shows the functional variation of $\delta_{\omega}$ and $\beta_{\omega}$ for a model protein over a wide energy range from $30 \mathrm{eV}$ to $30 \mathrm{keV}$. Clearly, both $\delta_{\omega}$ and $\beta_{\omega}$ are very small but especially for hard X-rays above $10 \mathrm{keV}$, the absorption decrement $\beta_{\omega}$ is about three orders of magnitude smaller than $\delta_{\omega}$. This small deviation of $n_{\omega}$ from unity motivates the ansatz

$$
\Psi_{\omega}(\vec{r})=\exp (i k z) \cdot \Psi_{s}(\vec{r})
$$

\footnotetext{
${ }^{2}$ Since the wavelength is smaller than the diameter of the atoms, tremendous variations occur on length scales given by $\lambda$. Nevertheless, the properties describing the effects of a large number of atoms usually vary much more slowly.
} 


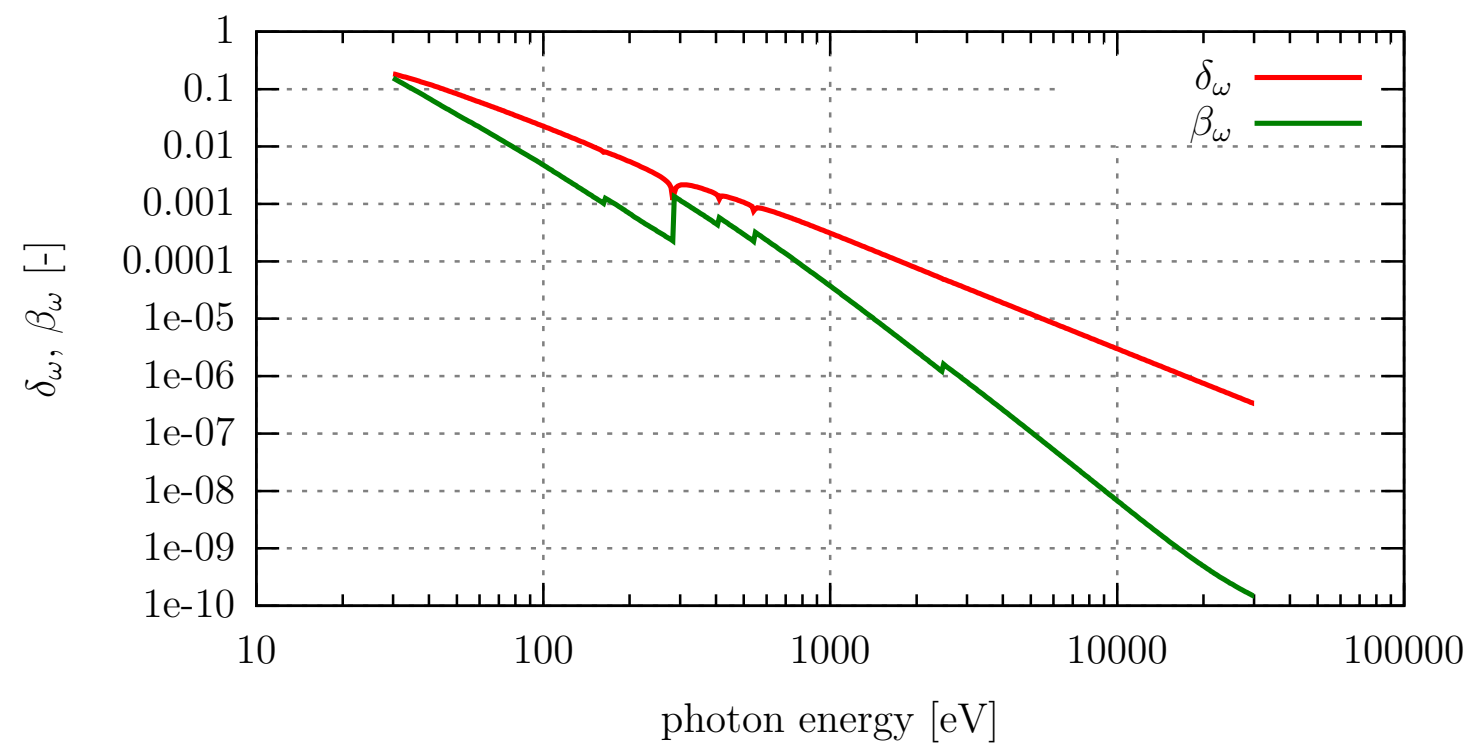

Figure 3.1: Phase-shift $\delta_{\omega}$ and absorption decrement $\beta_{\omega}$ for the model protein [31] $\mathrm{H}_{50} \mathrm{C}_{30} \mathrm{~N}_{9} \mathrm{O}_{10} \mathrm{~S}_{1}$ with a density of $1.35 \mathrm{~g} / \mathrm{cm}^{3}$. In the regime of X-rays above $5 \mathrm{keV}, \beta_{\omega}$ is more than two orders of magnitude smaller than $\delta_{\omega}$. Sudden changes in the lines originate from material-specific quantum mechanical absorption edges. Data from [32].

for the Helmholz equation in matter. The incident illumination $\exp (i k z)$ is given by a plane wave, which becomes only slightly modified by the well-behaving envelope $\Psi_{s}$ originating from the weak signal of all 'scattering' atoms. Inserted in equation (3.10), one obtains

$$
\left[2 i k \frac{\partial}{\partial_{z}}+k^{2}\left(n_{\omega}^{2}(\vec{r})-1\right)\right] \Psi_{s} \approx 0 .
$$

Since $\Psi_{s}$ varies only slowly in space, the remaining second derivative $\nabla^{2} \Psi_{s}$ has been neglected. Then, the so-called exit wave directly behind an object that is situated between $z=-z_{0}$ and $z=0$ is given by

$$
\Psi_{s}(x, y, z=0) \approx \Psi_{s}\left(x, y, z=-z_{0}\right) \exp \left(\frac{k}{2 i} \int_{-z_{0}}^{0} 1-n_{\omega}^{2}(\vec{r}) \mathrm{d} z\right) .
$$

The exit wave only depends on the values along undisturbed straight ray-paths parallel to the $z$-direction through the volume, as sketched in Fig. 3.2. With $n_{\omega}^{2} \approx 1-2\left(\delta_{\omega}-i \beta_{\omega}\right)$ one arrives at the projection approximation

$$
\begin{aligned}
\Psi_{s}(x, y, z=0) & \approx \Psi_{s}\left(x, y, z=-z_{0}\right) \exp \left(-i k \int_{-z_{0}}^{0} \delta_{\omega}(\vec{r})-i \beta_{\omega}(\vec{r}) \mathrm{d} z\right) \\
& =\Psi_{s}\left(x, y, z=-z_{0}\right) \exp \left(-i k \mathcal{P}\left[1-n_{\omega}(\vec{r})\right]\right) .
\end{aligned}
$$




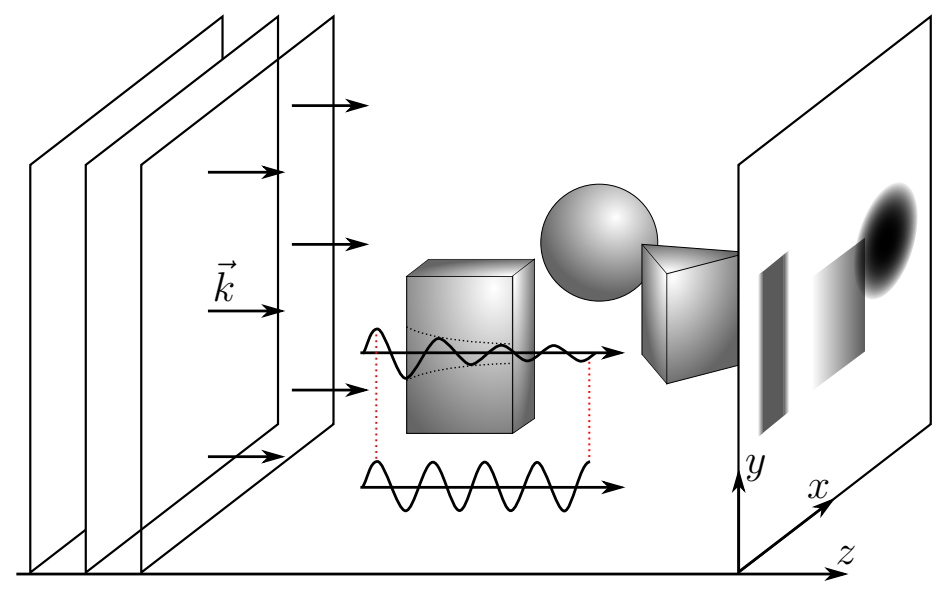

Figure 3.2: In the projection approximation, the incoming wave is represented by normal rays $(\| \vec{k})$, traversing the object unscattered. The 'exit wave' directly behind the object depends only on the absorption and phase shift along each of the rays.

For convenience, the projection operator

$$
\mathcal{P}[f(\vec{r})]:=\int_{\Omega} f(\vec{r}) \mathrm{d} z
$$

has been introduced, where the integration path is restricted to the support $\Omega(\vec{r}):=$ $\left\{\vec{r} \in \mathbb{R}^{3} \mid f(\vec{r}) \neq 0\right\}$ of the sample. With this result, the intensity $I \propto\left|\Psi_{s}\right|^{2}$ behind a homogeneous material of thickness $d$ is given by

$$
I(x, y, 0)=I_{0} \exp (-\underbrace{2 k \beta_{\omega}}_{=: \mu_{\omega}} \cdot d)
$$

The exponential decay with the object's thickness is the well-known Lambert-Beer-law with the absorption coefficient $\mu_{\omega}$.

For negligible non-linear effects in a static object, where the wave-matter interaction is assumed to be weak and non-magnetic, we finally obtain a description of the process similar to classical ray-optics, i.e. straight lines traversing the object. This is the foundation of medical X-ray imaging as well as of many X-ray microscopy techniques $[3,27]$ and will be used throughout this work. However, the presented approximations also point to the limitations of those techniques. As will be shown later, the requirement for small phase-shifts and weak absorption within the material is crucial for many applications. 


\subsection{Projections from different perspectives}

The projection approximation provides a direct "look" through objects but is clearly not sufficient to reveal their three dimensional structure. In a projection with a parallel beam (plane wave front, all $k$-vectors parallel) as defined in Eq. (3.14), all information about the $z$-distribution of the sample's features is lost. Tomography offers a method to overcome this limitation: The basic idea is to use a set of projections from different perspectives to reconstruct the three dimensional values of $n(\vec{r})$. As we will see, the rotation of the object - or the optical path - around one axis is sufficient to obtain a complete set and is the common strategy in practical applications. Generally, the principle of tomography is not limited to the case of parallel projections. In fact, most setups use diverging fan- or cone-beam geometries as produced by conventional X-ray tubes [27]. In contrast to the parallel beam geometry, this results in a slightly different magnification of object features close to and far from the source. However, this effect is usually too small to be exploited for $3 \mathrm{~d}$ positioning of features. Instead, the reconstruction relies on the same basic principles as summarised in the following sections for the most basic case of parallel projecion [25, 27].

\subsubsection{The Fourier Slice Theorem}

(a)

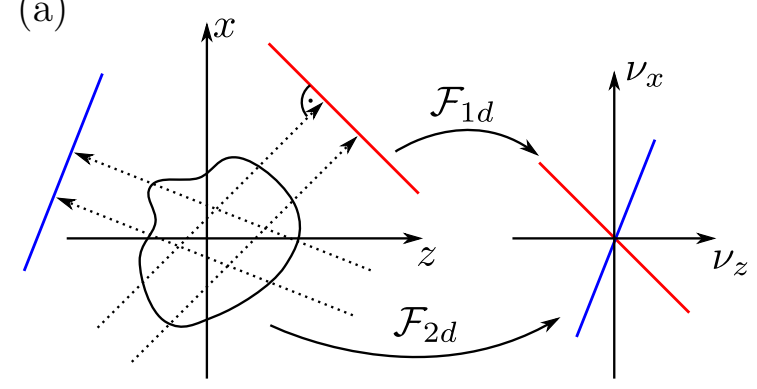

real space

Fourier space (b)

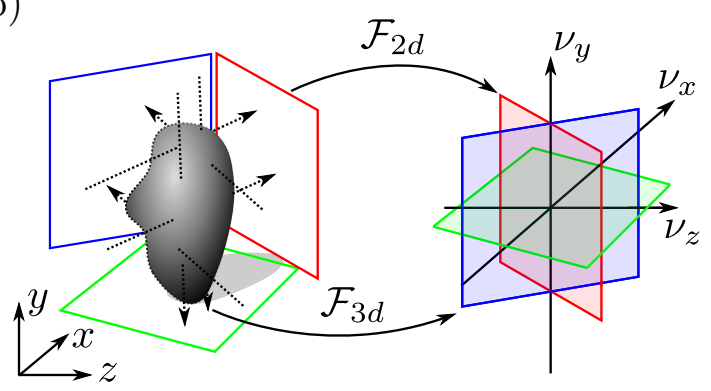

real space

Fourier space

Figure 3.3: The Fourier Slice Theorem in 2d (a) and 3d (b). The FT of a projection can be identified with a central slice through the FT of the sample with the same orientation.

The principle of tomography can be well understood by considering the projection operation in Fourier space. Due to the reciprocal character of the FT the global realspace integration can be expected to be local in Fourier space. Indeed, the (2d) FT of the projection

$$
\mathcal{F}_{2 d}(\mathcal{P}[f(\vec{r})])\left(\nu_{x}, \nu_{y}\right):=\int_{-\infty}^{\infty}\left[\int_{-\infty}^{\infty} f(\vec{r}) \mathrm{d} z\right] \exp \left(-i\left(\nu_{x} x+\nu_{y} y\right)\right) \mathrm{d} x \mathrm{~d} y
$$


can be identified as the central slice $\left(\nu_{z}=0\right)$ of the object's (3d) FT

$$
\mathcal{F}_{3 d}[f(\vec{r})]\left(\nu_{x}, \nu_{y}, \nu_{z}=0\right):=\left.\iint_{-\infty}^{\infty} \int_{\infty} f(\vec{r}) \exp \left(-i\left(\nu_{x} x+\nu_{y} y+\nu_{z} z\right)\right) \mathrm{d} x \mathrm{~d} y \mathrm{~d} z\right|_{\nu_{z}=0}
$$

This observation clearly holds for all orientations of the sample and is known as the Projection-Slice-Theorem or Fourier-Slice-Theorem (FST) [25-27]:

The ( $n$-1-dimensional) FT of a projection can be identified with a central slice through the (n-dimensional) FT of the object, orthogonal to the direction of projection.

In this context, "orthogonal" means that for a projection along an axis (z-axis here), the central slice is perpendicular to the corresponding Fourier space direction $\left(\nu_{z}\right.$ in this example). The FT defines the object as a superposition of infinitely extended frequency modes $\exp (i \vec{\nu} \cdot \vec{r})$. Every mode whose wave vector $\vec{\nu}$ is not exactly perpendicular to the axis of projection oscillates in this direction and therefore vanishes in the integration. Some consequences of this finding can be seen in the sketch in Fig. 3.3 for the $n=2$ and $n=3$-dimensional case. Firstly, all slices share the origin in Fourier space as their central point, which represents the mean value of the function $f$. This expresses the physical principle that the overall absorption and phase shift of a sample is independent of its orientation. More importantly, the FST provides a scheme for the full reconstruction of the object. The Fourier space can be sampled by central slices derived from projections along different directions. Then, $f$ can be obtained easily by an inverse FT. For that purpose, rotation around only one axis orthogonal to the optical path over a range of $180^{\circ}$ is sufficient and convenient, but not essential.

\subsubsection{The Radon transform}

In the following, we choose the $y$-coordinate as the axis of rotation. A generalised projection for the projection angle $\alpha$, i.e. along the path $x \cos \alpha-z \sin \alpha=s, s \in \mathbb{R}$ can be defined as

$$
\mathcal{P}_{\alpha}[f(\vec{r})](s, y):=\int_{-\infty}^{\infty} \int_{-\infty}^{\infty} f(\vec{r}) \delta_{D}(x \cos \alpha-z \sin \alpha-s) \mathrm{d} x \mathrm{~d} z .
$$

$\delta_{D}$ denotes the DiRAC delta distribution, not to be confused with the phase decrement $\delta_{\omega}$. As illustrated in Fig. 3.4, the discussed case having the axis of rotation perpendicular to the projection axis $z$ reduces to a $2 \mathrm{~d}$ problem. For convenience, we drop the $y$ coordinate but keep in mind that the object is represented by a stack of slices $y=$ const. which can be treated independently. Eq. 3.20 is called the Radon transform of $f(\vec{r})$ in honour of JOHANN RADON who introduced it in a 1917 article [19].

There are different ways to represent the set of projections, the so-called Radon space or projection space. Fig. 3.4 depicts representations in polar coordinates and the more commonly used Cartesian $(\alpha, s)$ coordinates. Since the Radon transform of a feature 


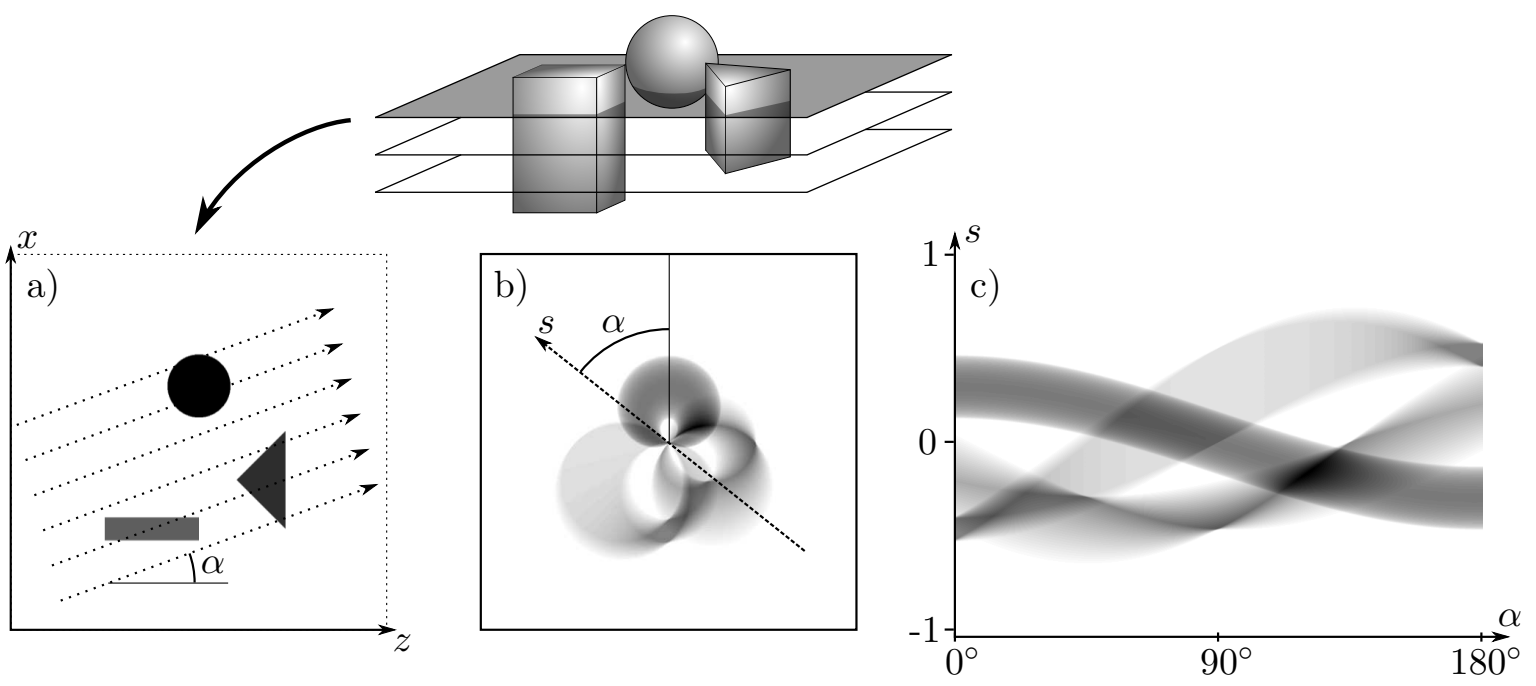

Figure 3.4: The projections $\mathcal{P}_{\alpha}[f(\vec{r})]$ of the phantom (a) form the Radon space. It can be depicted in polar coordinates (b) but is commonly presented in Cartesian coordinates as shown in (c). Since a feature of the phantom describes a sine curve in this representation, it is called a sinogram.

at $\vec{r}=(x, z)$ results in the sine curve $x \cos \alpha-z \sin \alpha$ in the Cartesian representation, the latter is called a sinogram [27].

The analytical radon transformation can be obtained for more complex bodies as well. In case of an ellipse it is given by [25]

$$
\begin{aligned}
\mathcal{P}[\text { ellipse }](\alpha, s) & =\frac{2 \varrho A B}{a(\alpha)^{2}} \sqrt{a(\alpha)^{2}-s^{2}} \\
\text { with } a(\alpha)^{2} & =A^{2} \sin ^{2} \alpha+B^{2} \cos ^{2} \alpha .
\end{aligned}
$$
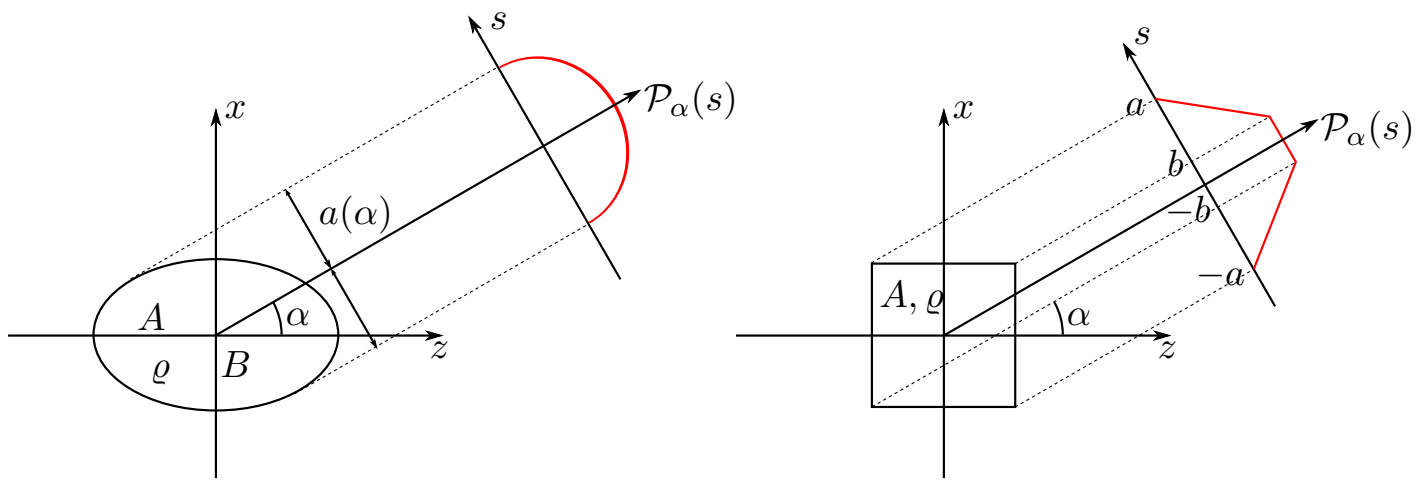

Figure 3.5: Simple shapes like ellipses or squares can be projected analytically. 


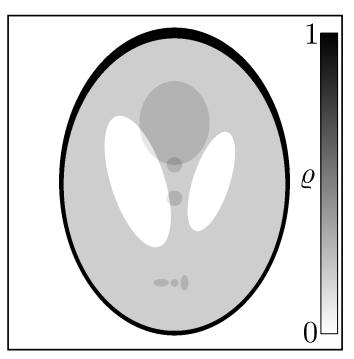

Figure 3.6

Here, $\varrho$ denotes the constant function value inside the elliptic region, as illustrated in Fig. $3.5 ; A$ is the semi-major axis while $B$ denotes the semi-minor axis. If the body is shifted with respect to origin, the projections in the sinogram are shifted according to the corresponding sine function. Due to the linearity of the projection, more complex objects can be composed as a sum of such primitives and the Radon transform is then given analytically by the sum of all individual projections. This is used for example in the Shepp and Logan "head phantom" depicted in Fig. 3.6. Exclusively consisting of ellipses, it serves as a ground-truth in simulations. A detailed description can be found in [25].

Even more important for later discretisation in a basis of pixels is the projection of a single square. As sketched in Fig. 3.5, in general it is given by the symmetric trapezoid

$$
\mathcal{P} \text { [square] }(\alpha, s)=\varrho A \cdot\left\{\begin{array}{cl}
(a+s) /\left(a^{2}-b^{2}\right), & \text { if }-a \leq s<-b \\
1 /(a+b), & \text { if }-b \leq s \leq b \\
(a-s) /\left(a^{2}-b^{2}\right), & \text { if } \quad b<s \leq a \\
0 & \text { else. }
\end{array}\right.
$$

with the area $A$, the function value $\varrho$ within the square, $a=\sqrt{A / 2} \sin \left(\alpha^{\prime}\right), b=$ $\sqrt{A / 2} \cos \left(\alpha^{\prime}\right)$ and $\alpha^{\prime}=(\alpha \bmod \pi / 4)+\pi / 4$.

\subsection{Direct tomographic reconstruction}

RADON showed in [19] that a function can be uniquely reconstructed from its representation in projection space. For this inversion, the FST already provides a practical scheme: First, the projection space is transformed to the object's Fourier space representation. Second, an inverse FT provides the reconstruction in real space. In the specific case of a sinogram, a Fourier transform along the $s$-direction has to be followed by a conversion to polar coordinates. A detailed mathematical description of this scheme can be found in all common textbooks on tomography [25-27].

In this section, we will approach the topic guided by the challenges of discretisation. In real experiments, only a finite number of projections with discrete (band) limited sampling are available. Moreover, an interpolation from polar coordinates of the central slices to discrete Cartesian coordinates is required. These issues will then lead to a sophisticated numerical reconstruction method to invert the radon transform - the famous filtered backprojection (FBP). 


\subsubsection{Discrete sampling}

In the most basic case, discrete projections consist of identically sized quadratic pixels arranged on a Cartesian grid and the reconstruction grid is chosen of similarly sized pixels, too [25]. By requiring the Fourier space to be sampled in full, a criterion for the minimum number of projections can be derived. To guarantee that every pixel in reciprocal space is measured, the distance of neighbouring central slices must not be larger than one pixel. Thus, if projections with a radial size of $n_{y}$ pixels are acquired for equidistant angular steps $\Delta \alpha$ covering a range of $180^{\circ}$ with the axis of rotation in the centre of each image,

$$
N_{s}=\left\lceil n_{y} \frac{\pi}{2}\right\rceil
$$

projections are necessary for full sampling with $\lceil x\rceil=\min (n \in \mathbb{Z} \mid m \leq x)$. In practice, much fewer angles are tolerable but lead to specific undersampling artefacts. Numerically, projections can be obtained from an object representation as sketched in Fig. 3.7. The value of each object pixel is distributed to a maximum of three projection pixels. The pixel is projected according to Eq. (3.22) and its value is weighted by the integral of the resulting trapezoid in the projection pixel interval.

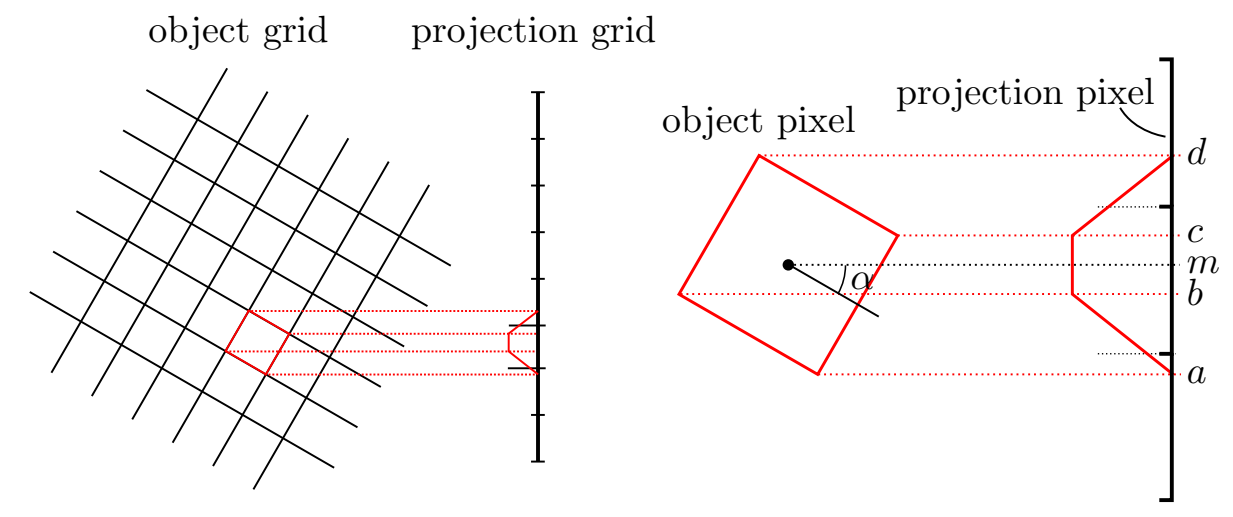

Figure 3.7: In a discrete projection, the value of each object pixel is weighted by the integral of its trapezoidal projection (Eq. (3.22)) in the projection pixel interval.

\subsubsection{The backprojection}

Computationally, graphics are represented in Cartesian coordinates. In addition, software and algorithms like the FFT are implemented and optimised for this basis. Therefore, the central slices have to be transformed from polar coordinates to a Cartesian basis. As shown in chapter 2.2.3, the required interpolation in discrete Fourier space is a non-trivial task, involving a convolution with a $2 \mathrm{~d}$ sinc function considering the limited real space. Hence, it can be numerically more efficient to implement this step directly in real space. There, a wide range of interpolation methods are available which do not introduce too many artefacts. Subsequently, we will rely on bilinear interpolation which shows a higher accuracy compared to the simple nearest-neighbour method but does not introduce negative values from a strictly positive input as can occur in bicubic and higher-order interpolation. 


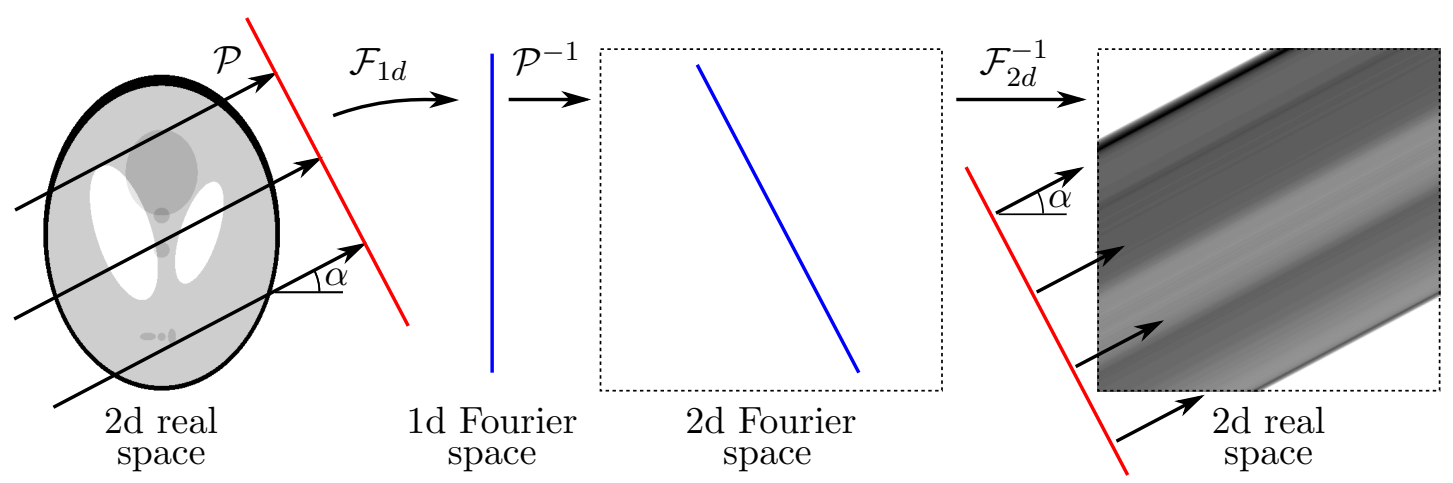

Figure 3.8: The analytical inverse FT of a properly oriented central slice in $2 \mathrm{~d}$ Fourier space is the original projection "smeared back" to the reconstruction volume along the same direction it was projected previously.

Since a central slice is infinitely thin in analytical reciprocal space, its equivalent in real space gets extended infinitely in the appropriate direction. Thus, the inverse FT of a single central slice properly oriented in $2 \mathrm{~d}$ Fourier space shows constant values along the original direction of projection as illustrated in Fig. 3.8. In the orthogonal direction, by definition, it shows the same course as the projection. Therefore, instead of adding the central slices to Fourier space, the reconstruction can be carried out by "smearing back" the projections into real space along the same direction as they where projected. This procedure is termed "backprojection" (BP), abbreviated by the operator $\mathcal{P}_{\alpha}^{-1}$ and illustrated in Fig. 3.9 for different numbers of projections. Details about the implementation can be found in appendix 9.1.

The numerical BP inherently takes into account the limitations of a discretised version of real space. This becomes obvious when considering the BP of a single projection: Except for special cases (e.g. $\alpha=0^{\circ}$ ), the periodic repetition of the numerical BP is discontinuous. Thus, in addition to the central slice expected from the analytical theory, a DFT of the numerical BP reveals further features in Fourier space as detailed in Chap. 2.2.3. These patterns are equivalent to the signals that are obtained by a convolution with the interpolation sinc-kernel in Fourier space, as explained in Chap. 2.2.3.

It should be mentioned that methods are available to circumvent the problem of Fourier space interpolation, such as the linogram algorithm based on the chirp-z-transformation [27] or equally sloped tomography, which relies on the pseudo-polar FFT [33]. These schemes require specific sampling of the projections at non-equidistant sampling points or angles, respectively. Since the problem of interpolation can be accurately solved by $\mathrm{BP}$ in real space, no significant increase in quality can be expected from these techniques. However, efficient implementations of these methods may have an advantage in reconstruction speed. 


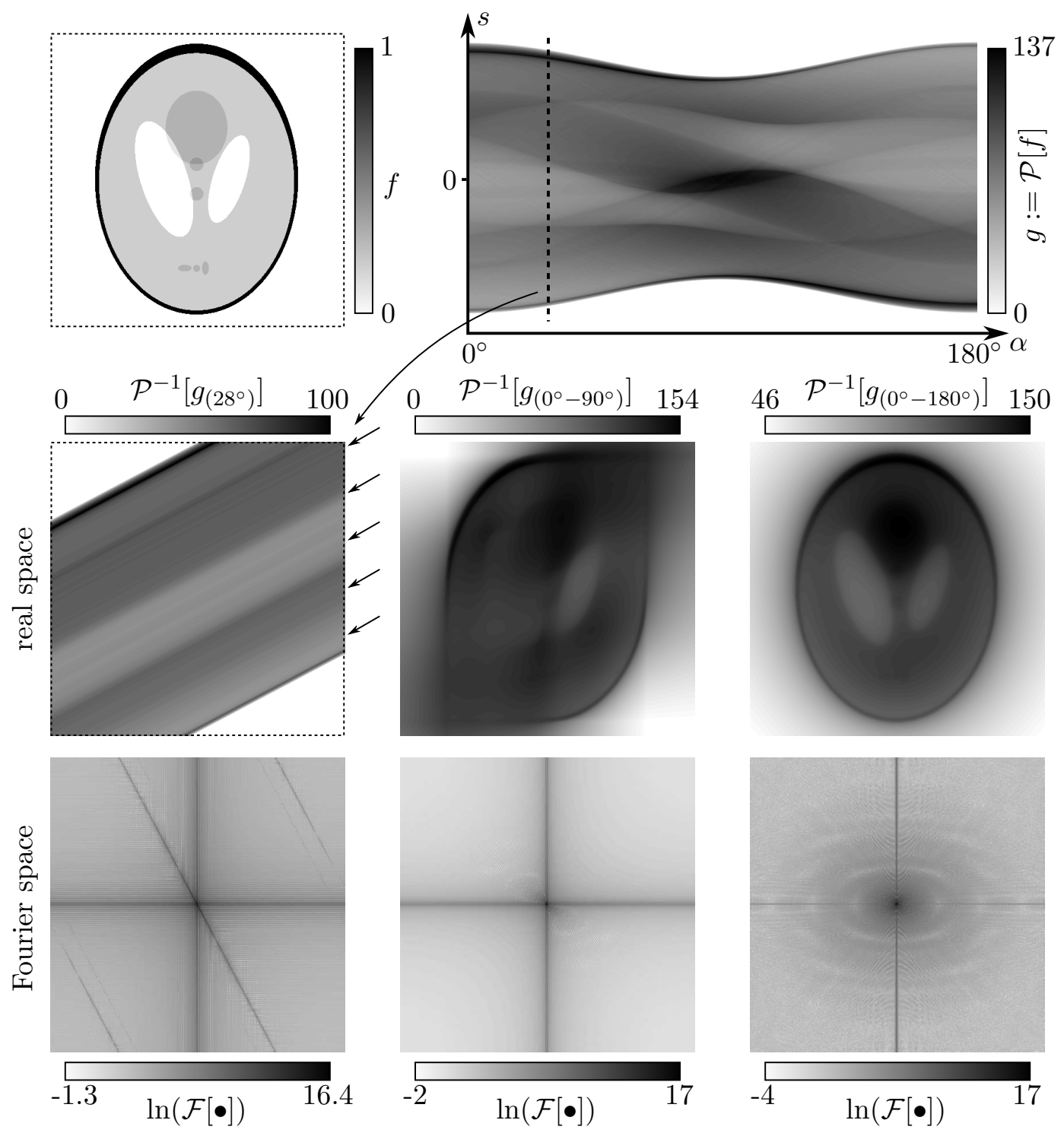

Figure 3.9: Backprojection (BP) for different numbers of projections. A phantom $(512 \times 512 \mathrm{px})$ has been projected to $N=805$ equidistant angles for full sampling. The FT of the numerical BP of a single projection (bottom left) shows not only a central slice but also patterns arising from the limited rectangular extent of the real-space pixel grid. In the central column, the BP of the first half of the sinogram is depicted while a complete $\mathrm{BP}$ is shown on the right.

\subsubsection{Filtered layergram and filtered backprojection}

The backprojections shown in Fig. 3.9 are still far from being correct reconstructions. The reason for this can be studied ideally in terms of the backprojection of a single point as sketched in Fig. 3.10. The backprojection lines cross at the actual position of the point, creating the highest signal there. But they are not restricted to this position. Instead, the signal is spread over the whole reconstruction space, decaying with $1 /(2 \pi r)$ as the density of lines decreases with distance $r$ from the point's position. What can be seen here directly as the Point-spread-function (PSF) of the backprojection is a 

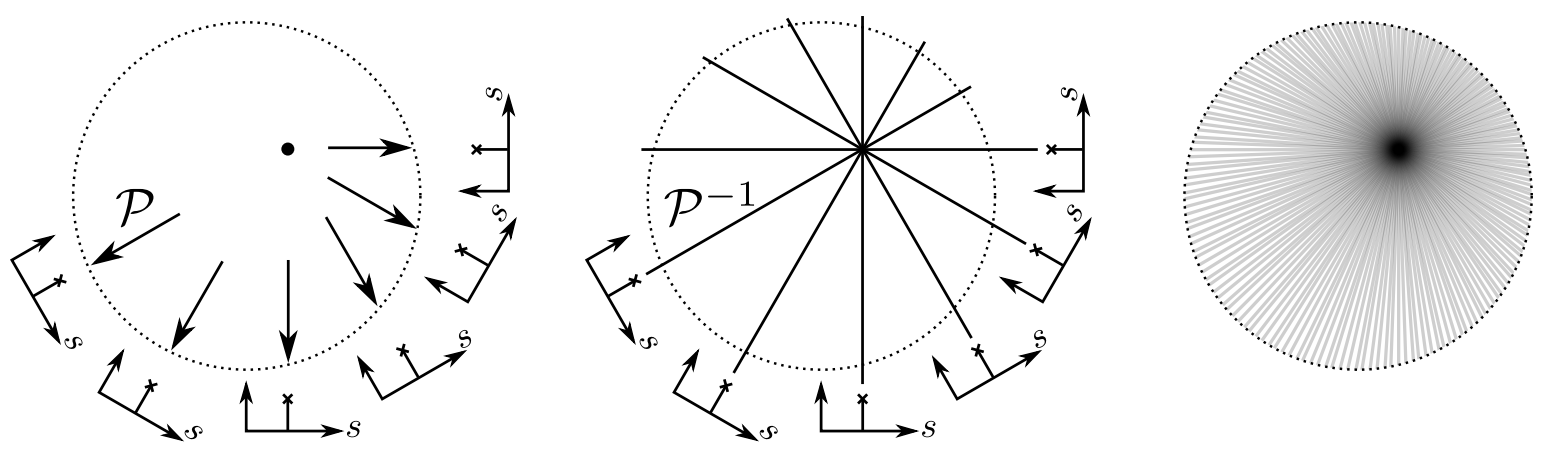

Figure 3.10: Projections of a single point are a single peak (left). The direct backprojection of such signals results in lines, crossing at the position of the original point (centre). For a large number of projections, the reconstructed points shows a decay $\propto 1 / r$ (right) which can be interpreted as the point-spread function of the process.

convolution kernel limiting the reconstruction quality of all samples. In Fourier space, this convolution can be inverted by a division with the transformed kernel.

In the present case, the Fourier transform of the PSF and the deconvolution can be obtained easily without explicit calculations, since in Fourier space the problem becomes immediately apparent. The sample density of central slices is not uniform but rises towards the centre, leading to an overweighting of this region. Thus, a weighting by the inverse sample point density $(2 \pi|\nu| / N)$ for $N$ central slices compensates for the artefacts. This high-pass Fourier filter $\propto|\nu|$ is usually termed ramp filter. Mathematically, it appears as the Jacobian determinant stemming from the change of basis between polar and Cartesian coordinates [25-27].

If the filter $\propto|\vec{\nu}|$ is applied directly in 2d Fourier space after the backprojection step, the result is called a filtered layergram. Alternatively, according to the FST, each projection can be filtered by $|\nu|$ before the backprojection step, a procedure termed "filtered backprojection" (FBP) [27]. Both approaches are identical in the analytical formulation ${ }^{3}$. However, depending on the order of filtering and interpolation in a numerical implementation discrepancies occur in the additional signals appearing next to the central slices during discrete BP. Historically, the filtered backprojection has been the preferred scheme. Advantages are, for example, the smaller memory usage and the availability of a coarse reconstruction already during the measurement (in particular when projections are acquired first in coarse angular steps, which are subsequently refined).

Fig. 3.11 recapitulates the FBP reconstruction strategy. First, the projection (or the entire sinogram, if already available) is filtered in Fourier space and transformed back to real space. Second, the filtered projections are backprojected into the reconstruction volume along the directions they where recorded originally. The line-profile in Fig. 3.11 still shows deviations from the reference known as "cupping" or "dishing" [25]. This error can be reduced by zero-padding before filtering, i.e. embedding the projections in a larger grid filled with zero-valued pixels outside the original grid. As we will see, this only alleviates the negative effects, but does not remove their cause.

By using the analytical ramp filter, the band-limited nature of the projections has

$\overline{3}$ The identity is clearly visible e.g. in the Riess-potential introduced on the first pages of [26]. 


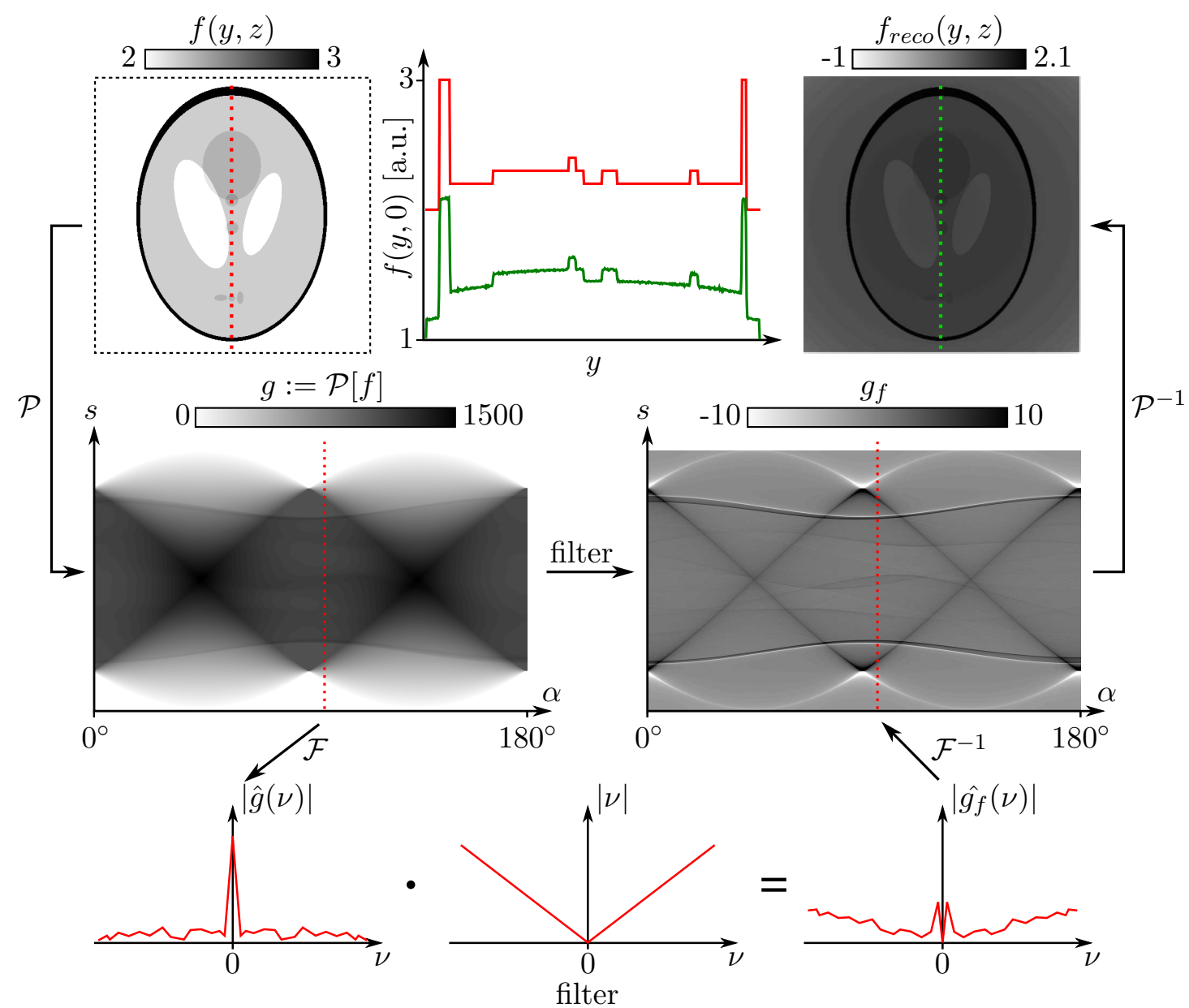

Figure 3.11: The principle of filtered backprojection (FBP). The projections $g(\alpha, s):=$ $\mathcal{P}[f(y, z)]$ are transformed to Fourier space, multiplied with a filter function $\propto|\nu|$ and transformed back to real space (performing a deconvolution). Then, the filtered projections are backprojected to the reconstruction volume. With the ramp filter derived analytically (see text) so-called "dishing" artefacts can be observed, leading to a convex curvature of the reconstructed function. The profiles along the dashed lines in the upper row allow for a quantitative comparison. Notice that a constant offset of magnitude 2 has been added to the phantom for better visibility of this effect.

been ignored again. The DFT of the discrete projections is limited to a maximum frequency, which implicitly also limits the filter to the sampled region. However, in discrete Fourier space this limit cannot be expressed easily, since the signal is considered to be repeated periodically. Nevertheless, the band-limit can be incorporated in a convolution kernel by analytically transforming a windowed Fourier filter to real space. Let the ramp filter in analytical Fourier space be limited by the unit box $\Pi(\nu)=1$ for $|\nu| \leq 1 / 2$; 0 otherwise. The corresponding real space convolution kernel is then given by

$$
f_{\text {ana. }}(s)=\mathcal{F}^{-1}[\Pi(\nu) \cdot 2 \pi|\nu| / N](s)
$$




$$
\begin{aligned}
& =\frac{1}{N} \int_{-1 / 2}^{1 / 2}|\nu| \exp (i \nu s) \mathrm{d} \nu \\
& =\frac{1}{N} \cdot \frac{2 \cos \left(\frac{s}{2}\right)+s \sin \left(\frac{s}{2}\right)-2}{s^{2}} .
\end{aligned}
$$

With this, the band-limitation is being considered, and as a second step, the kernel has to be discretised. According to the DFT, we have to select sampling points corresponding to harmonic modes in Fourier space of the form $M_{j}=m_{j} \exp (i \cdot 2 \pi j \nu)$ with $j \in \mathbb{N}$ full oscillations in the interval $|\nu| \leq 1 / 2$. Hence, we find the discrete sample points at the positions $s_{j}=j \cdot 2 \pi$ for $-N / 2 \leq j \leq N / 2$. Fig. 3.12 shows the analytical convolution kernel $f_{\text {ana. }}$ from Eq. (3.24) next to the discretized version $f_{\text {dis. }}$. At the sample points $s_{j}$ one obtains the simple scheme [25]

$$
f_{\text {dis. }}(j)=\frac{1}{N} \begin{cases}\frac{1}{4} & \text { if } j=0 \\ 0 & \text { if } j \text { even } \\ -\frac{1}{\pi^{2} j^{2}} & \text { if } j \text { odd. }\end{cases}
$$

For an efficient implementation of the discrete convolution, the band-limited and discretised kernel is transformed to Fourier space again to be applied there as a multiplicative filter (notice that it has to be padded to a power of 2 for a proper discrete convolution). In Fig. 3.12 the result is compared to the analytical ramp function, clearly showing a deviation at the origin. Importantly, the new filter does not force the origin value to zero, removing the dishing artefacts and preserving the mean value of the reconstruction. In contrast, zero-padding before filtering only limits the incorrect "analytical" filter values to a smaller fraction of pixels.
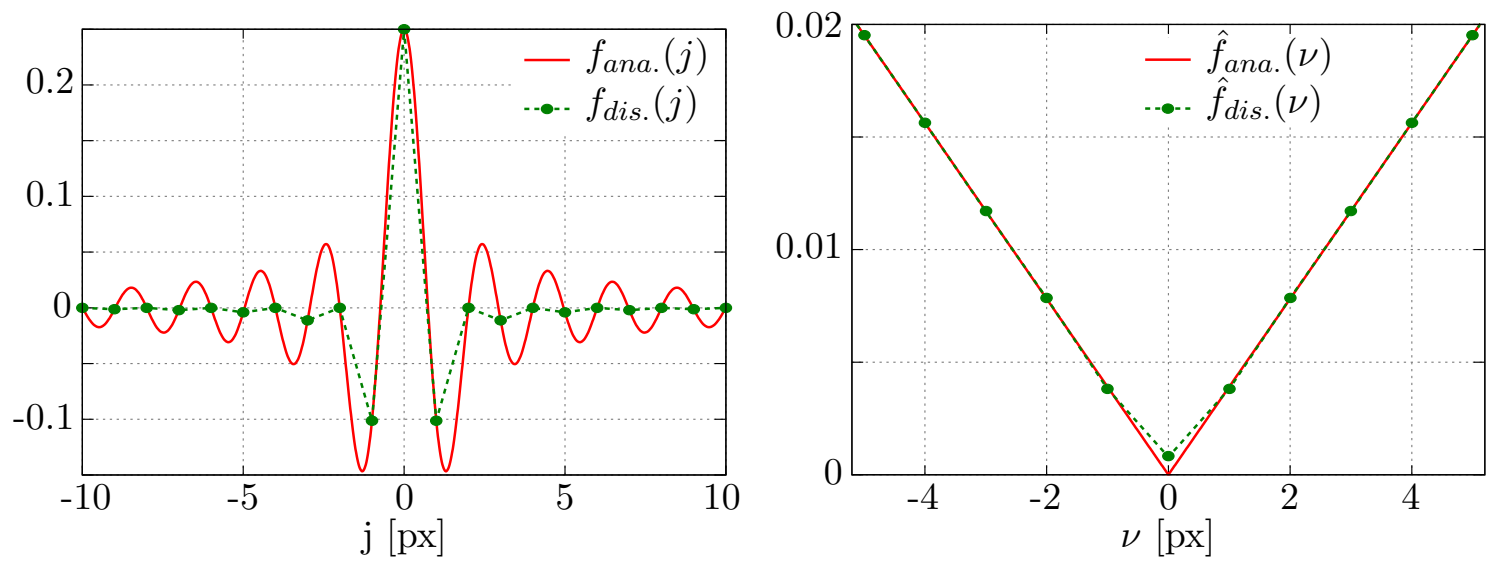

Figure 3.12: The Ram-Lak filter. Both the analytical and discretised versions of the convolution kernel that is equivalent to a band-limited ramp filter are shown on the left. If the discrete kernel is transformed to Fourier space und compared with the ramp-function (right), a deviation at the origin can be noticed.

The discretised filter is called Ram-Lak after G. N. RAmachandran and A. V. LAKSHMINARAYANAN [27]. It is often combined with additional Fourier-filters like a VON-HANN, HAMMING or sinc window to reduce the influence of high frequency noise 
that would otherwise be amplified by the ramp. The most common filter today is the so-called Shepp-Logan-Filter, combining the ramp-function with a windowed sinc [27]. An overview of discretised filters is given in [27]. A recent development is the attempt to adapt the filter for non-equidistant or sparse sampling as well as including certain forms of a priori knowledge [34].

In summary, problems associated with discretisation not only favour a real-space implementation of the backprojection but also require a filter that is adapted specifically to the sampling. To underline the importance of this concept, the FBP is sometimes termed convolution backprojection. As we will see again in the next chapters, operations in Fourier space always have to be carried out with care.

\subsection{Algebraic reconstruction techniques}

The Fourier-slice theorem suggests that the central slices of an object - and thereby also the projections - are largely independent of each other. The only exception is the common central point in Fourier space, representing the mean value of object and projections. For any other point, an adjustment in Fourier space would only affect the containing slice and thus only become visible in the corresponding projection. But this suggestion is deceptive, as already indicated by the interpolation in section 3.3.2. There, the limited nature of real space leads to additional signals in Fourier space, coupling a central slice to almost all others. Indeed, this is not just an artefact from discretisation. If the object $f$ is compactly supported by $\Omega$, the multiplication with any well behaving function $g$ that equals one everywhere inside $\Omega$ and has arbitrary values outside yields

$$
f(\vec{r}) \cdot g(\vec{r})=f(\vec{r})
$$

since all arbitrary values from $g$ are multiplied with zeros. In Fourier space, this translates to the convolution

$$
\hat{f}(\vec{\nu}) \star \hat{g}(\vec{\nu})=\hat{f}(\vec{\nu})
$$

For every compact object, an infinite number of such convolution kernels $\hat{g}$, whose application must not change the values of $\hat{f}$, can be found. This introduces a strong dependency between the slices. In order not to violate the support condition, values of a single central slice cannot be changed arbitrarily but the changes have to be compensated in virtually all other slices according to the convolution kernel.

In real space, such a strong dependency is expected intuitively. If a common object is rotated only a little, the projections are expected to be nearly identical as well. Distinct features can be seen in every projection and form sine curves in the sinograms. Mathematically, all these dependencies are summarised in the so-called Helgason-Ludwig consistency condition (HLCC) [35-37] opening extensive possibilities for data alignment, error compensation and retrieval of lost projections. Moreover, it is this dependency between projections that enables the use of iterative algebraic reconstruction techniques as a flexible and highly extendable approach for the inversion of the RADON transform, as we will see in this chapter. 


\subsubsection{Consistency conditions}

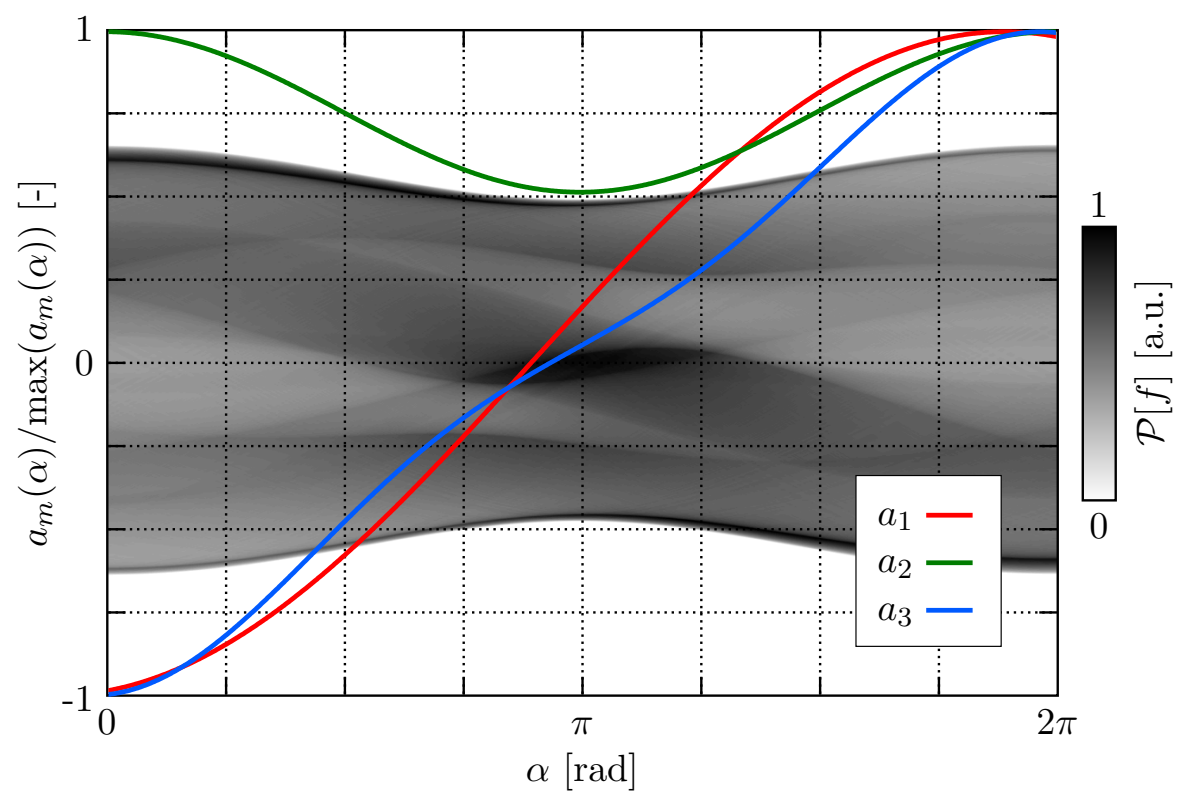

Figure 3.13: First modes of a sinogram obtained from the ShePp and Logan headphantom.

The Helgason-Ludwig consistency condition imposes three rules on a sinogram derived from a physical object by an undisturbed Radon-transformation. Firstly, Picard's condition has to be fulfilled, which implies that the norm of the reconstruction from a consistent sinogram is finite. Next, the projection-moment theorem has to be obeyed. Let $g(s, \alpha)$ be the sinogram of a function $f(x, z)$ compactly supported within the unit circle. Then

$$
a_{m}(\alpha)=\int_{-1}^{1} g(s, \alpha) s^{m} \mathrm{~d} s
$$

defines the $m$-th moment of $g(s, \alpha)$ with respect to $s$ for $m \in \mathbb{N}_{0}$. Expressed as a Fourier series, one obtains

$$
a_{m}(\alpha)=\frac{1}{2 \pi} \sum_{l=-\infty}^{\infty} a_{m l} \exp (i l \alpha)
$$

with the coefficients

$$
a_{m l}=\int_{0}^{2 \pi} a_{m}(\alpha) \exp (-i l \alpha) \mathrm{d} \alpha .
$$

For a consistent sinogram, there must be

$$
a_{m l}=0 \text { for }|l|>m \text {. }
$$




\subsection{Algebraic reconstruction techniques}

Finally, from the symmetry of the sinogram, i.e. $g(s, \alpha+\pi)=g(-s, \alpha)$, it also follows that

$$
a_{m l}=0 \text { for }|l|+m \text { odd. }
$$

Fig. 3.13 visualises the meaning of the last two conditions. The zeroth moment is the mean value of all projections and has to be constant in $\alpha$. This is equal to the observation that the sum of all voxel values is always the same, independent of the order of summation as carried out by different projections. The first moment is proportional to the centre of mass of the projections. Since by definition, this point is the projected centre of mass of the object, it is expected to show a sine function with exactly one period in the $360^{\circ}$ of the sinogram. Indeed, for $m=1$ the HLCC allows only non-zero Fourier coefficients for $l= \pm 1$. This observation is useful for alignment of real data, since the centre of mass is often more robust to track than object-specific features varying from sample to sample. The second moment is required to show exactly two full oscillations in the sinogram while the third moment is the first represented by more than two non-zero Fourier coefficients.
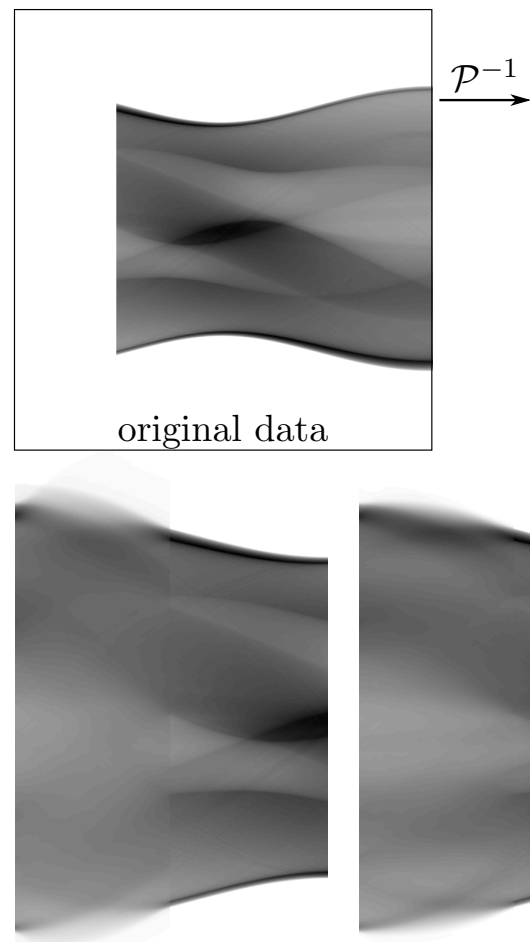

10 iterations

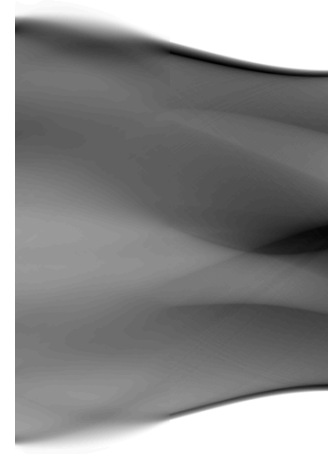

100 iterations
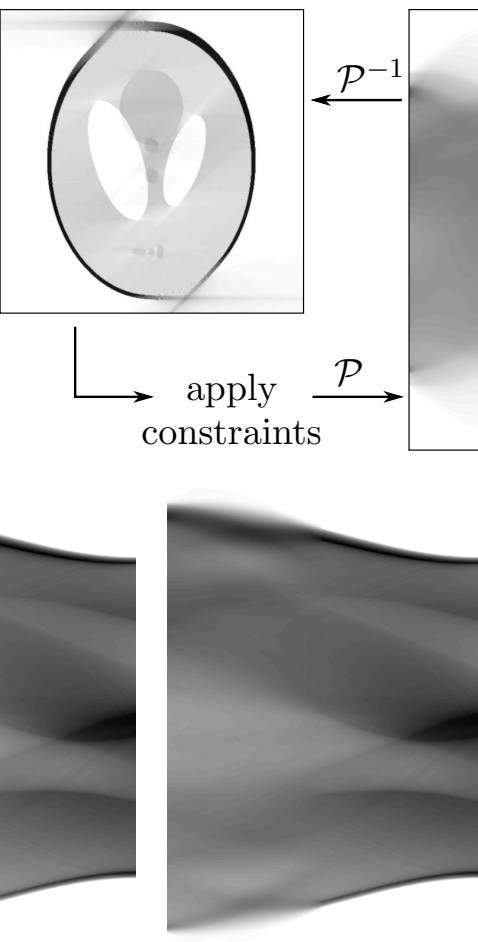

1000 iterations

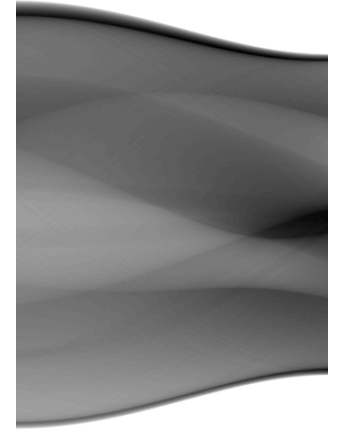

ground truth

Figure 3.14: Sinogram retrieval using an iterative reprojection scheme. The missing $25 \%$ of the initial sinogram can be approximated by iteratively reconstructing the object from the data (FBP) followed by applying some constraints (in this case, positivity) and reprojecting the missing angles.

Unfortunately, the moment problem, i.e. reconstructing $g$ from the series of moments $a_{m}$ is a non-trivial task. Nevertheless, enforcing the consistency can be exploited to retrieve non-measured parts of the sinogram. Fig. 3.14 shows results of an iterative strategy called Iterative Reconstruction-Reprojection (IRR) [38]. The first $25 \%$ of the 
sinogram have been deleted and the reconstruction using FBP is appropriately disturbed. However, if the reconstruction is numerically projected to the missing angles ("reprojection"), the coarse shape of the sinogram can be retrieved because of the strong dependency between all projections. With this improved sinogram, a better FBP reconstruction can be obtained and reprojected again. Iteratively, the reconstructed sinograms approach a true reproduction of the original values.

For faster convergence, a positivity constraint has been applied to the reconstructed objects in the shown example. Since a negative absorption cannot occur, all nonphysical negative values have been set to zero in each iteration. Additional knowledge like the support of the object or the used materials can be considered to improve the reconstruction. This scheme shows that undersampling with respect to the number of projections as well as non-equal angular steps can be compensated to a certain degree. In the end, even projections of an object in an experimentally inaccessible direction can be approached. Importantly, iterative reprojection procedures of this kind make it possible to not only retrieve missing projections but also reconstruct the object itself.

\subsubsection{Iterative reconstruction techniques}

While developing the first CT brain scanner, HounsfiELD was unaware of previous direct reconstruction solutions from projection data. He developed an iterative reconstruction scheme considering the problem as a system of linear equations [39]

$$
\mathcal{P}: \quad \vec{b}=\mathbf{A} \vec{v} .
$$

Here, $\vec{v}$ contains the $M_{x} \cdot M_{z}$ pixel values of the object voxels, projected by the matrix A to obtain the $N \cdot M_{x}$ sinogram values $\vec{b}$. The matrix $\mathbf{A}$ is usually too large for a direct inversion. Additionally, if the projections are distorted by noise or the system of equations is underdetermined (e.g. $N<M_{z}$ ), only an approximate solution can be obtained, e.g. by minimizing the difference $\chi^{2}:=|\mathbf{A} \vec{v}-\vec{b}|^{2}$ between reprojections and measured data. For this purpose, the method of Kaczmarz has been established $[25,27,40]$ :

$$
\vec{v}_{l+1}=\vec{v}_{l}+\frac{b_{i}-\left(\vec{a}_{i}^{T} \cdot \vec{v}_{l}\right)}{\left(\vec{a}_{i} \cdot \vec{a}_{i}^{T}\right)} \vec{a}_{i}^{T}
$$

Here, $a_{i}^{T}$ is the transposed $i$-th row of the matrix $\mathbf{A}$ with $i=l \bmod \left(M_{x} \cdot N\right)+1$ or chosen randomly from the $M_{x} \cdot N$ rows. An initial estimation $\vec{v}_{0}$ can be chosen arbitrarily but a good approximation of the solution is obviously preferred. The robustness of this method is often superior to competing algorithms like the method of conjugated gradients [41]. Simultaneously with Hounsfield's solution, Gordon, Bender and HERMAN proposed implementations of KACZMARZ' method for tomography called Algebraic Reconstruction Techniques (ART). Expressed by the operators $\mathcal{P}$ and $\mathcal{P}^{-1}$, the recorded projections $\Phi_{\alpha}$ and the reconstruction $f$ are defined as before, their "direct multiplicative method" is given by

$$
f_{l+1}=f_{l} \cdot \mathcal{P}_{\alpha}^{-1}\left[\frac{\Phi_{\alpha}}{\mathcal{P}_{\alpha}\left[f_{l}\right]}\right]
$$



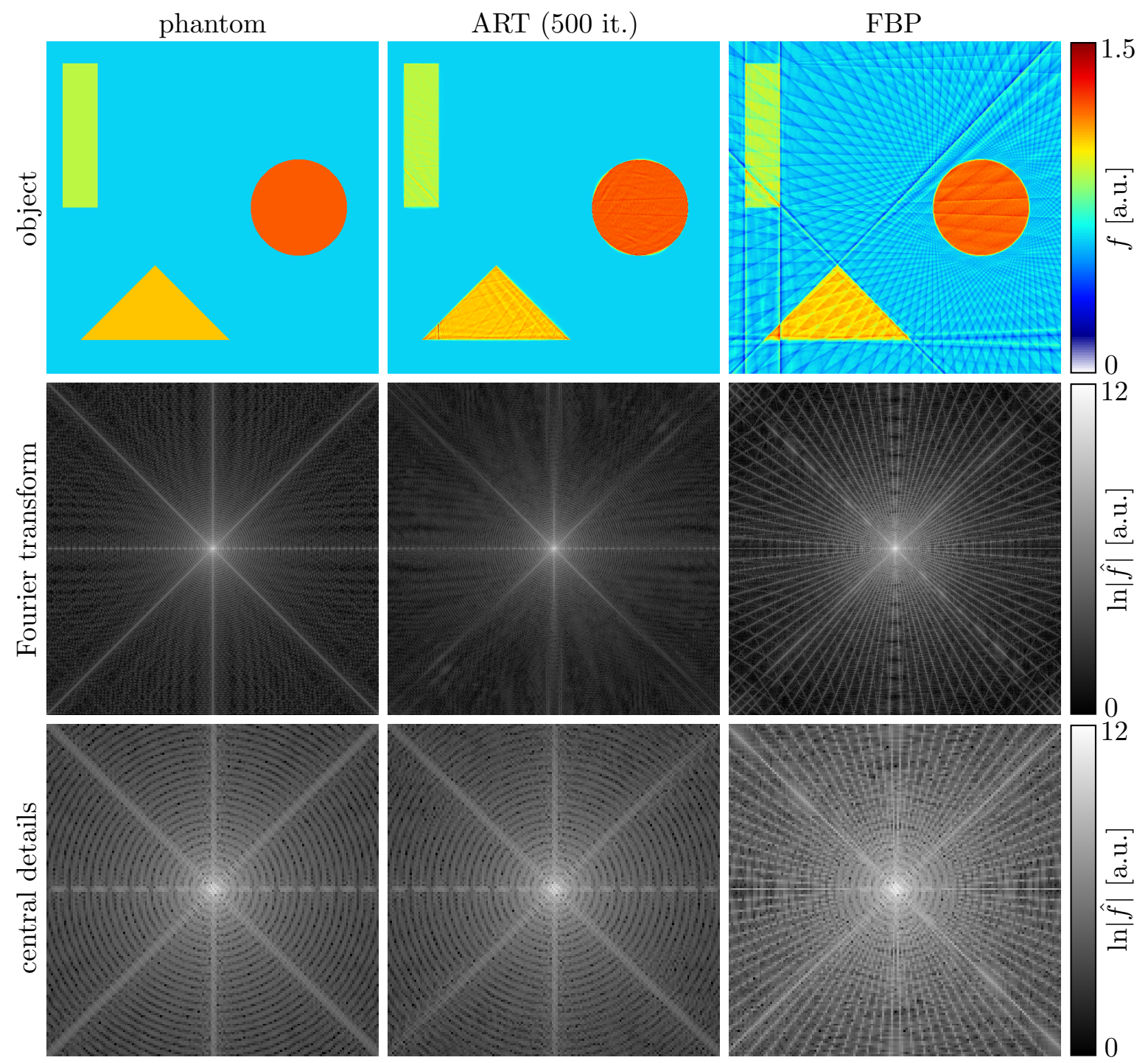

Figure 3.15: Comparison of $\mathrm{FBP}$ and ART reconstruction from $N=45$ equidistant projections. ART clearly creates superior results with fewer artefacts. The price is a much longer run-time of the iterative algorithm. In the Fourier transform of the FBP result, the central slices are well recognisable. Exploiting consistency, ART is capable to reconstruct the missing information and fill the gaps to a certain degree.

The "direct additive method" is defined as

$$
f_{l+1}=f_{l}+\max \left(\mathcal{P}^{-1}\left[\Phi_{\alpha}-\mathcal{P}_{\alpha}\left(f_{l}\right)\right] / \eta_{\alpha}, 0\right)
$$

Here, correct scaling is guaranteed by the function $\eta_{\alpha}=\mathcal{P}_{\alpha}\left(f_{l} / f_{l}\right)$ providing the number of projected voxels for each projection pixel. The divisions are point-wise. Numerous refinements of these schemes are available. They all have in common the basic principle that the guess $f_{l}$ is projected and compared to the desired values $\Phi_{\alpha}$. Subsequently, a correction factor is derived from the deviations and applied to the reconstruction volume to obtain an improved approximation $f_{l+1}$. In Eq. (3.35) and (3.36), the correction 
factors are applied with the help of the backprojection. Instead of the projections, the correction values are "smeared back" into the volume, adjusting the values of all voxels "touched" by a backprojected pixel equally. The steps are repeated for all projections. The choice of order has a large influence on the speed of convergence. Nearby projections only demand nearly the same correction factors and thus convergence gets improved for a sequence with large angular steps between successive projections [25].

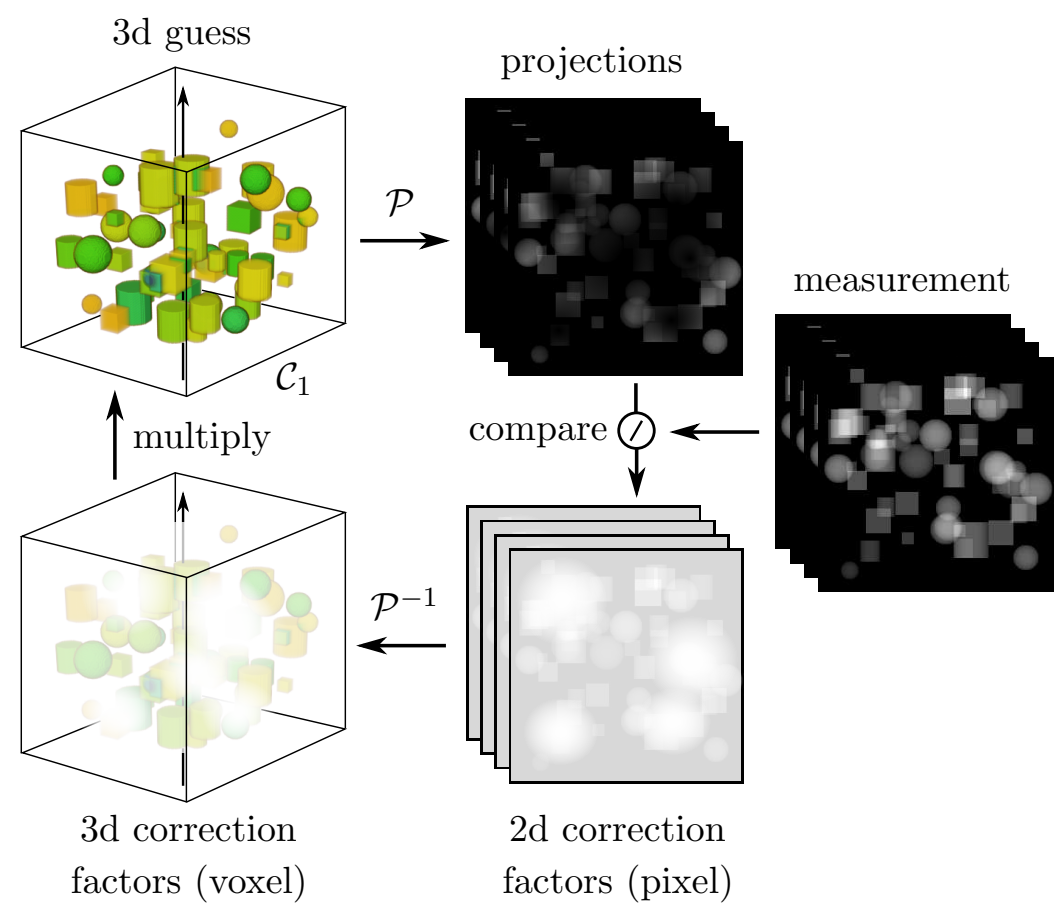

Figure 3.16: Principle of ART. A guess of the object is projected numerically to the experimental angles. The projections are compared to the measurement to obtain correction factors which are subsequently backprojected and applied to the guess. See text for details.

Due to the dependency of all projections, the volume satisfies the condition $\mathcal{P}_{\alpha}\left[f_{l}\right]=\Phi_{\alpha}$ only along the latest corrected direction $\alpha$. Along other directions discrepancies to the desired values appear even if they have been corrected only a few steps earlier, requiring the procedure to be applied iteratively. As in IRR, this leads to a reconstruction of the gaps in an undersampled Fourier space as illustrated in Fig. 3.15 which compares the result of eq. (3.35) with a FBP of the same data.

Since even a single wrong projection can heavily disturb the reconstruction process in the above schemes, more robust methods have been developed. A popular example among these is the Simultaneous Reconstruction Technique (SIRT). It first averages the correction values from all projections before the object values get adjusted by one of the above rules. However, the quality improvement requires a considerably longer reconstruction time compared to the projection-by-projection updates in common ART [25]. Importantly, all iterative methods allow imposing constraints on the reconstruction. While positivity is implicitly included in ART, the support, a maximum absorption, sparsity in the material composition, and so on can be enforced in each iteration as well. This is not only beneficial for the speed of convergence but may also 
3.4 Algebraic reconstruction techniques

allow reconstructing reasonable objects from poor data like noisy and undersampled sinograms. 



\section{Wave field propagation}

While the projection approximation allows describing wave-matter interaction in terms of geometrical optics, the subsequent propagation is determined by the wave character of the radiation. For increasing distances between object and detector, the monochromatic - and thus coherent - wave field will display interference effects which ultimately dominate its shape. The origin of their appearance is of great interest. The weak absorption of hard X-rays by soft matter such as biological tissue only yields small brightness variations in the exit waves with a weak signal-to-noise ratio. Due to freespace propagation, not only these small absorption signals develop to well measurable effects. The intensity pattern at a distant detection plane will instead be dominated by the much stronger phase-shifting properties of the sample. Free-space propagation can thus be used as an instrument to gain phase contrast, i.e. encoding previously invisible phase shifts into well measurable brightness variations. Optimally, large distances between sample and detector are required.

This chapter presents a summary of the theoretical framework of coherent wave propagation as detailed in [29] and [3]. For a precise description of the development of a wave field, we first obtain the derivation of the so-called diffraction integral and an equivalent formulation in Fourier space. Second, a large part of this chapter is dedicated to the numerical implementation of propagation and its associated problems. In this context, an instructive method to overcome the most dominant numerical artefacts is presented. The chapter closes with a description of how to combine tomography and propagation, opening up further possibilities of data treatment and simulation by introducing a $3 \mathrm{~d}$ propagated object.

\subsection{The diffraction formula}

\subsubsection{Fresnel-Kirchhoff theory}

The behaviour of monochromatic electromagnetic waves in a volume of free space is governed by the HeLMHOLTz equation (HE)

$$
\left[k^{2}+\nabla^{2}\right] \Psi(\vec{r})=0
$$

introduced in Eq. (3.7). In physics, it is often possible to draw conclusions about the occurrences in a given volume from observations of its closed border. In this respect, we can solve the problem by rewriting it as an integral over the surface of the volume 
of interest. For this purpose, we introduce an additional Green's function $G(\vec{r})$ next to the wave field $\Psi(\vec{r})$, both solutions to the same HE. Then,

$$
\Psi \cdot G-G \cdot \Psi=0 .
$$

By applying the HE $k^{2} G=-\nabla^{2} G$ and $k^{2} \Psi=-\nabla^{2} \Psi$ one obtains

$$
\Psi \nabla^{2} G-G \nabla^{2} \Psi=0 .
$$

The integral of this equation over the volume $V$ must equal zero, too, and allows to apply Green's Theorem

$$
\iiint_{V} \Psi \nabla^{2} G-G \nabla^{2} \Psi \mathrm{d} V=\iint_{S} \Psi \frac{\partial G}{\partial n}-G \frac{\partial \Psi}{\partial n} \mathrm{~d} s=0,
$$

transforming the expression to an integral over the volume's surface $S$. The partial derivatives $\partial / \partial n$ have to be carried out along the direction $\vec{n}$ normal to the surface. To proceed, the auxiliary function $G$ has to be chosen in a way that the equation can be solved for $\Psi$. KIRCHHOFF proposed a spherical wave

$$
G(\vec{r})=\frac{\exp \left(i k\left|\vec{r}-\vec{r}_{0}\right|\right)}{\left|\vec{r}-\vec{r}_{0}\right|}
$$

with amplitude 1 , originating from $\overrightarrow{r_{0}}$ within the volume $V$. Due to the singularity at $\vec{r}_{0}$, we omit this point in $V$ and modify the surface $S=S_{V}+S_{\varepsilon}$ such that $S_{\varepsilon}$ is a concentric sphere around $\vec{r}_{0}$ with the radius $\varepsilon$, as sketched in Fig. 4.1. Then, for all $\vec{r}$ on $S_{\varepsilon}$ we have

$$
G\left(\vec{r}_{@ S_{\varepsilon}}\right)=\frac{\exp (i k \varepsilon)}{\varepsilon} \quad \text { and } \quad \frac{\partial G\left(\vec{r}_{\in S_{\varepsilon}}\right)}{\partial n}=\frac{\exp (i k \varepsilon)}{\varepsilon}\left(\frac{1}{\varepsilon}-i k\right)
$$

and in the limit $\varepsilon \rightarrow 0$ the surface $S_{\varepsilon}$ contributes [29]

$$
\lim _{\varepsilon \rightarrow 0}\left[\iint_{S_{\varepsilon}} \Psi \frac{\partial G}{\partial n}-G \frac{\partial \Psi}{\partial n} \mathrm{~d} s\right]=4 \pi \Psi\left(\vec{r}_{0}\right)
$$

With this result, the remaining integration over the volume's outer surface $S_{V}$ can be written as

$$
\Psi\left(\vec{r}_{0}\right)=\frac{1}{4 \pi} \iint_{S_{V}} \frac{\partial \Psi}{\partial n} \cdot\left(\frac{\exp \left(i k\left|\vec{r}-\overrightarrow{r_{0}}\right|\right)}{\left|\vec{r}-\overrightarrow{r_{0}}\right|}\right)-\Psi \cdot \frac{\partial}{\partial n}\left(\frac{\exp \left(i k\left|\vec{r}-\overrightarrow{r_{0}}\right|\right)}{\left|\vec{r}-\overrightarrow{r_{0}}\right|}\right) \mathrm{d} s .
$$

This equation is known as the integral theorem of Helmholtz and Kirchhoff [29] and finally allows to derive $\Psi$ at any point in the volume from its values and normal derivative at the surface. As shown in chapter 3.1.2, a projection only provides a so- 


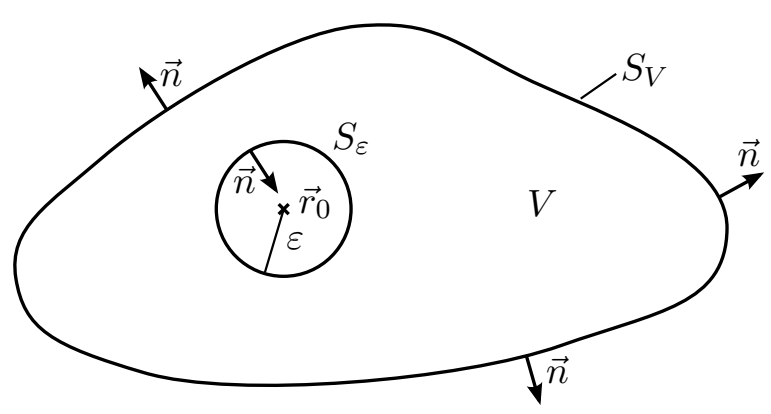

Figure 4.1: Integration surface $S=S_{V}+S_{\varepsilon}$ for a GREEN's function $G$ given by an outgoing spherical wave originating from $\vec{r}_{0}$.

called exit wave $\Psi$ on a plane $S_{1}$ but not on a closed surface. Again, it is useful to divide the integration surface $S_{V}=S_{1}+S_{2}$ into the known plane $S_{1}$ and a spherical dome $S_{2}$ closing the surface as depicted in Fig. 4.2. It can be shown [29] that the contribution of $S_{2}$ vanishes if its radius $R$ increases to infinity, provided that $\Psi$ satisfies the Sommerfeld radiation condition

$$
\lim _{R \rightarrow \infty} R\left(\frac{\partial \Psi}{\partial n}-i k \Psi\right)=0
$$

uniformly with respect to all directions. For this purpose, $\Psi$ has to vanish at least as fast as an outgoing spherical wave. The radiation condition also prohibits incoming spherical waves originating at infinity and moving towards $S_{1}$, ensuring a unique solution [42]. With

$$
\frac{\partial}{\partial n}\left(\frac{\exp \left(i k\left|\vec{r}-\overrightarrow{r_{0}}\right|\right)}{\left|\vec{r}-\overrightarrow{r_{0}}\right|}\right)=\frac{\exp \left(i k\left|\vec{r}-\vec{r}_{0}\right|\right)}{\left|\vec{r}-\overrightarrow{r_{0}}\right|}\left(i k-\frac{1}{\left|\vec{r}-\overrightarrow{r_{0}}\right|}\right) \cos \left(\vec{n},\left(\vec{r}-\vec{r}_{0}\right)\right)
$$

we find the general equation

$$
\Psi\left(\vec{r}_{0}\right)=\frac{1}{4 \pi} \iint_{S_{1}} \frac{\exp \left(i k\left|\vec{r}-\vec{r}_{0}\right|\right)}{\left|\vec{r}-\vec{r}_{0}\right|}\left[\frac{\partial \Psi}{\partial n}-\Psi\left(i k-\frac{1}{\left|\vec{r}-\vec{r}_{0}\right|}\right) \cos \left(\vec{n},\left(\vec{r}-\vec{r}_{0}\right)\right)\right] \mathrm{d} s
$$

for arbitrarily curved surfaces $S_{1}$.

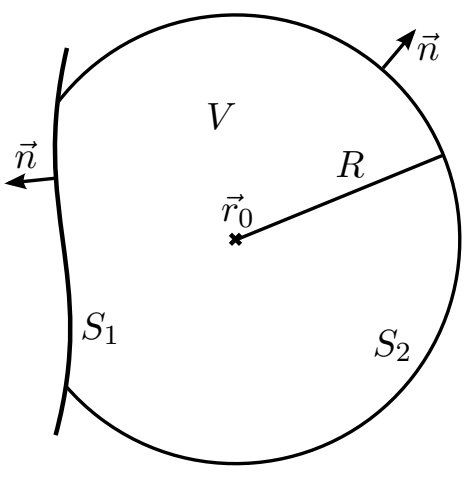

Figure 4.2: The outer surface $S_{V}$ gets split into the (potentially curved) plane $S_{1}$ and a spherical dome around $\vec{r}_{0}$ closing the surface at $R \rightarrow \infty$. 
Eq. (4.11) provides a rigorous solution of the wave field if $\Psi$ and $\partial \Psi / \partial n$ are known everywhere on a single infinite plane. Unfortunately, in most cases, only parts of these values are available, e.g. a projection only provides $\Psi$ and not $\partial \Psi / \partial n$. Thus, often un-physical and mathematically deceptive boundary conditions are required to be satisfied, leading to inconsistent solutions. Famously, the theorem is used to calculate the field behind holes in a hypothetic perfectly absorbing screen. Within the holes, the illumination is considered undisturbed as if the screen would not be present. Directly behind the absorbing areas of the screen $\Psi$ and $\partial \Psi / \partial n$ are required to equal zero. These are KICHHOFF's boundary conditions for diffraction on a screen, ensuring convergence of the integrals. However, apertures modelled by step functions or even delta distributions for small holes do not satisfy the HE. Moreover, the vanishing of both $\Psi$ and its normal derivative on a finite interval behind the screen imply that the function is zero everywhere in space [29]. Hence, the field calculated by Eq. (4.11) with the given boundary conditions has to violate the same conditions on the screen (see e.g. [43] for a discussion of this problem).

\subsubsection{Rayleigh-Sommerfeld theory}

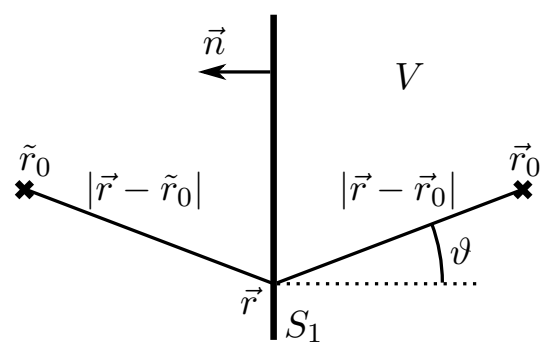

Figure 4.3: Method of images: The source point $\overrightarrow{r_{0}}$ of the spherical wave $G$ is mirrored at the flat screen $S_{1}$, yielding the origin $\tilde{r}_{0}$ of a second spherical wave. The difference of both waves is zero on the entire plane while for the sum of both waves the normal derivative equals zero.

Inconsistencies related to boundary conditions in Eq. (4.4) mainly arise because both $\Psi$ and the normal derivative $\partial \Psi / \partial n$ have to be provided. As introduced by SoMMERFELD in [44], for the special case of a flat plane $S_{1}$ this requirement can be relaxed by the choosing an alternative Greens' function

$$
G_{-}\left(\overrightarrow{r_{0}}\right)=\frac{\exp \left(i k\left|\vec{r}-\overrightarrow{r_{0}}\right|\right)}{\left|\vec{r}-\overrightarrow{r_{0}}\right|}-\frac{\exp \left(i k\left|\vec{r}-\tilde{r}_{0}\right|\right)}{\left|\vec{r}-\tilde{r}_{0}\right|}
$$

with $\tilde{r}_{0}$ being the point $\vec{r}_{0}$ mirrored at the screen $S_{1}{ }^{1}$. Again, we omit the point $\vec{r}_{0}$ in the volume $V$ and the limit $\varepsilon \rightarrow 0$ results in a contribution of $4 \pi \Psi\left(\overrightarrow{r_{0}}\right)$ from the surface $S_{\varepsilon}$. The contribution of $S_{2}$ vanishes for $R \rightarrow \infty$ if the Sommerfeld radiation condition

\footnotetext{
${ }^{1}$ Strictly speaking, $\tilde{r}$ "is located on the second sheet of a two-sheeted RIEMAnN surface so it does not interfere with the illuminated part of the field" [42].
} 
is satisfied. The crucial property of this choice for $G_{-}$is that it equals zero everywhere on the planar screen $S_{1}$. Thus, the final integration over this plane yields

$$
\Psi\left(\vec{r}_{0}\right)=-\frac{1}{4 \pi} \iint_{S_{1}} \Psi \frac{\partial G_{-}}{\partial n} \mathrm{~d} s
$$

The normal derivative of $G_{-}$on $S_{1}$ is given by

$$
\frac{\partial G_{-}}{\partial n}=2 \frac{\exp \left(i k\left|\vec{r}-\vec{r}_{0}\right|\right)}{\left|\vec{r}-\vec{r}_{0}\right|}\left(i k-\frac{1}{\left|\vec{r}-\vec{r}_{0}\right|}\right) \cos \left(\vec{n},\left(\vec{r}-\vec{r}_{0}\right)\right),
$$

leading to

$$
\Psi\left(\vec{r}_{0}\right)=-\frac{1}{2 \pi} \iint_{S_{1}} \Psi \frac{\exp \left(i k\left|\vec{r}-\vec{r}_{0}\right|\right)}{\left|\vec{r}-\vec{r}_{0}\right|}\left(i k-\frac{1}{\left|\vec{r}-\vec{r}_{0}\right|}\right) \cos \left(\vec{n},\left(\vec{r}-\vec{r}_{0}\right)\right) \mathrm{d} s .
$$

Eq. (4.15) is the first Sommerfeld solution to the diffraction problem. It yields the wave field for the situation that only $\Psi$ is known everywhere on a single, flat and infinite plane. A second SOMMERFELD solution can be obtained by the function

$$
G_{+}\left(\overrightarrow{r_{0}}\right)=\frac{\exp \left(i k\left|\vec{r}-\overrightarrow{r_{0}}\right|\right)}{\left|\vec{r}-\overrightarrow{r_{0}}\right|}+\frac{\exp \left(i k\left|\vec{r}-\tilde{r}_{0}\right|\right)}{\left|\vec{r}-\tilde{r}_{0}\right|}
$$

with the normal derivative $\partial G_{+} / \partial n$ equal to zero on the screen. An analogue derivation as above results in

$$
\Psi\left(\vec{r}_{0}\right)=\frac{1}{2 \pi} \iint_{S_{1}} \frac{\partial \Psi}{\partial n} \frac{\exp \left(i k\left|\vec{r}-\vec{r}_{0}\right|\right)}{\left|\vec{r}-\vec{r}_{0}\right|} \mathrm{d} s
$$

only depending on the normal derivative of $\partial \Psi / \partial n$ on $S_{1}$.

\subsubsection{Comparison of theories}

We now have a choice between three expressions for the propagated field. Curved surfaces can be treated by the KIRCHHOFF diffraction formula $\Psi_{K}$ in Eq. (4.11). In case of a flat screen the first and second SommERFELD solutions $\Psi_{S 1}$ in Eq. (4.15) and $\Psi_{S 2}$ in Eq. (4.17), respectively, provide the same correct result ${ }^{2}$ from less boundary conditions. A comparison reveals that the KIRCHHOFF formula is the arithmetic mean of the two Sommerfeld solutions,

$$
\Psi_{K}=\frac{1}{2}\left(\Psi_{S 1}+\Psi_{S 2}\right)
$$

Further similarities can be readily identified: All expressions formulate the propagated field as a convolution. Each point on the plane $S_{1}$ can be considered as the source of

\footnotetext{
$\overline{2}$ This can be easily verified e.g. by a numerical comparison of the wave fields obtained from the values on a plane illuminated by a single spherical wave.
} 
an outbound spherical wave with amplitude equal to the boundary value at its origin. This is close to the Huygens-Fresnel principle. However, a pure spherical wave only appears in the convolution with the normal derivative in Eq. (4.17). Without loss of generality we can chose the plane $S_{1}$ equal to $z=0$ and defined $r=\sqrt{x^{2}+y^{2}+d^{2}}$ to obtain the wave field in a parallel plane $z=d$ with a single operation

$$
\Psi_{\text {Som } 2}(x, y, z=d)=\left[\frac{\partial}{\partial z} \Psi(x, y, z=0)\right] *\left[\frac{1}{2 \pi} \frac{\exp (i k r)}{r}\right] .
$$

In $\Psi_{\text {Som } 1}$ the spherical wave is shaped by additional terms, including the so-called inclination or obliquity factor OF [29]

$$
\Psi_{\text {Som } 1}(x, y, z=d)=\Psi(x, y, z=0) *[\frac{1}{2 \pi} \frac{\exp (i k r)}{r}\left(\frac{1}{r}-i k\right) \underbrace{\frac{d}{r}}_{O F}] .
$$

The cosine has been rewritten as $\cos \vartheta=d / r$. Physically, the $O F$ prevents backpropagation of the wave field in the direction of its origin.

\subsection{Fresnel propagation}

As indicated by the convolution formula in Eq. (4.20), the problem of propagation can be formulated equivalently in Fourier space. In this section, a descriptive introduction is preferred over the rigorous mathematical derivation provided by textbooks, see e.g. $[3,29]$.

The inverse Fourier transform expresses the wave field $\Psi$ in a flat plane uniquely as a sum of infinitely extended oscillations

$$
\Psi(x, y)_{z=0}=\frac{1}{4 \pi^{2}} \int_{-\infty}^{\infty} \hat{\Psi}\left(\nu_{x}, \nu_{y}\right) \exp \left(i\left(\nu_{x} x+\nu_{y} y\right)\right) \mathrm{d} \nu_{x} \mathrm{~d} \nu_{y}
$$

of the electromagnetic field. Here, the plane $z=0$ has been chosen without loss of generality. If we assume monochromatic radiation with $|\vec{k}|=2 \pi / \lambda=$ const. propagating from the negative to the positive half-space $z>0$, a physical meaning can be assigned to these oscillations. The different wavelengths $\lambda_{\perp}=2 \pi /|\vec{\nu}|$ in the (2d) plane can only be explained if they are considered as slices through (3d) plane waves with the same wavelength $\lambda$ but different orientations. Vice versa, each oscillation in the (2d) plane can be extended to a (3d) plane wave with complex amplitude $\hat{\Psi}$ leaving in a unique direction $\vec{k}_{\vec{\nu}}$, as sketched in Fig. 4.4. The sum of these plane waves can be evaluated at any point in space. This is the basic idea of the Fresnel or angular-spectrum approach for propagation. 


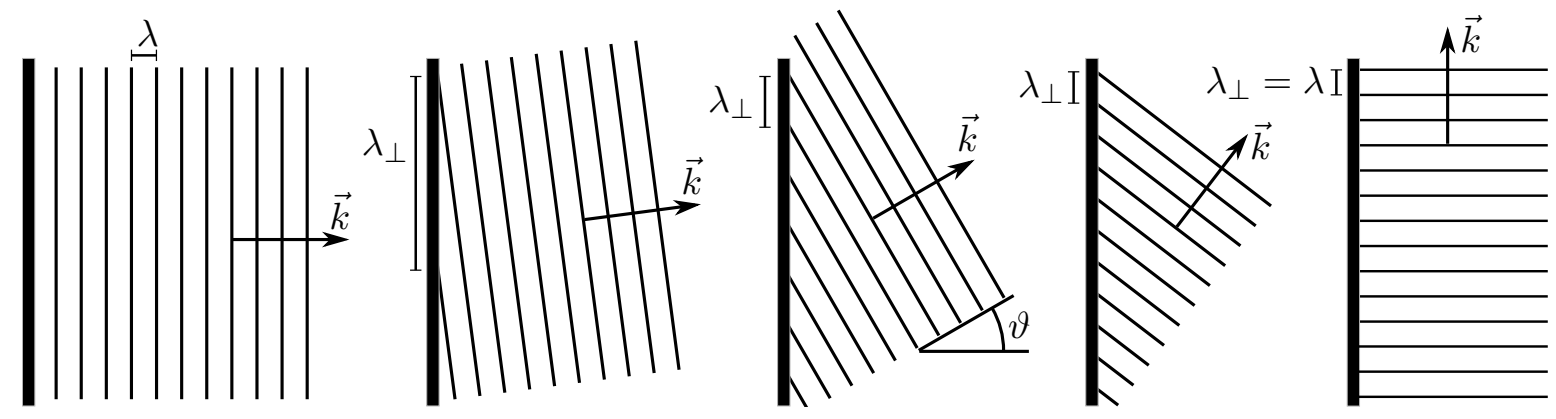

Figure 4.4: The angular spectrum of a wave field, sketched for a $1 \mathrm{~d}$ screen. The wave field on the screen can be expressed as a sum of oscillations with different wavelength $\lambda_{\perp}$, given by a Fourier transform. For monochromatic illumination, these oscillations can be uniquely identified as slices through plane waves with different orientations, defining the wave field everywhere in free space behind the screen.

Since we can identify the Fourier space coordinates $\nu_{x} \equiv k_{x}$ and $\nu_{y} \equiv k_{y}$ as components of the wave vector $\vec{k}$ with $k^{2}=k_{x}^{2}+k_{y}^{2}+k_{z}^{2}$, the corresponding plane wave is given by

$$
\Psi_{\vec{\nu}}(\vec{r})=\frac{\hat{\Psi}\left(\nu_{x}, \nu_{y}\right)}{4 \pi^{2}} \exp \left(i\left[\nu_{x} x+\nu_{y} y+z \sqrt{k^{2}-\nu_{x}^{2}-\nu_{y}^{2}}\right]\right)
$$

It crosses the plane at an angle

$$
\sin \vartheta=\frac{k_{\perp}}{k}=\frac{|\vec{\nu}|}{k}=\frac{\lambda}{\lambda_{\perp}} \quad \Rightarrow \quad \vartheta=\operatorname{asin}\left(\frac{\lambda}{\lambda_{\perp}}\right)
$$

so that waves corresponding to small wavelengths $\lambda_{\perp}$ in the plane get "diffracted" to large angles. The wave field $\Psi$ behind every plane is an angular spectral decomposition of $\Psi$ in the plane. The complete propagated wave field is given by

$$
\Psi(x, y, z)=\frac{1}{4 \pi^{2}} \int_{-\infty}^{\infty} \int_{\Psi}\left(\nu_{x}, \nu_{y}\right) \exp \left(i\left[\nu_{x} x+\nu_{y} y+z \sqrt{k^{2}-\nu_{x}^{2}-\nu_{y}^{2}}\right]\right) \mathrm{d} \nu_{x} \mathrm{~d} \nu_{y} .
$$

For the special case of propagation to a parallel plane at distance $z=d$, one obtains

$$
\begin{aligned}
\Psi(x, y)_{z=d} & =\frac{1}{4 \pi^{2}} \int_{-\infty}^{\infty} \int_{\mathcal{F}(\Psi)_{z=0}}^{\hat{\Psi}\left(\nu_{x}, \nu_{y}\right)_{z=0}} \underbrace{\exp \left(i d \sqrt{k^{2}-\nu_{x}^{2}-\nu_{y}^{2}}\right)}_{\chi^{d}\left(\nu_{x}, \nu_{y}\right)} \exp \left(i\left(\nu_{x} x+\nu_{y} y\right)\right) \mathrm{d} \nu_{x} \mathrm{~d} \nu_{y} \\
& =\mathcal{F}^{-1}\left[\mathcal{F}(\Psi)_{z=0} \cdot \chi^{d}\right] .
\end{aligned}
$$


Hence, in Fourier space the propagation can be expressed by a multiplication with the so-called chirp function ${ }^{3}$

$$
\chi^{d}=\exp \left(i d \sqrt{k^{2}-\nu_{x}^{2}-\nu_{y}^{2}}\right)
$$

as sketched in Fig. 4.5. It can be shown [46] that the inverse Fourier transform of this function

$$
\begin{aligned}
\mathcal{F}^{-1}\left[\chi^{d}\right] & =\frac{1}{4 \pi^{2}} \iint_{-\infty}^{\infty} \chi^{d}\left(\nu_{x}, \nu_{y}\right) \exp \left(i\left(\nu_{x} x+\nu_{y} y\right)\right) \mathrm{d} \nu_{x} \mathrm{~d} \nu_{y} \\
& =\frac{1}{2 \pi} \frac{\exp (i k r)}{r} \cdot \underbrace{\frac{d}{r}}_{\cos (\vartheta)}\left(\frac{1}{r}-i k\right)
\end{aligned}
$$

with $r=\sqrt{x^{2}+y^{2}+d^{2}}$ equals the first SOMMERFELD convolution kernel as derived in Eq. (4.15) and (4.20) for the same boundary conditions. In [3] equivalence is shown for the paraxial approximations. The angular spectrum approach hence considers all features of the wave field, including the obliquity effect and evanescent waves. The latter can be seen even more directly compared to the real-space formulation: Evanescent waves appear for $k_{\perp}>k$, requiring a forbidden ${ }^{4}$ angle of radiation of $\vartheta>90^{\circ}$. In this case, the exponent of the chirp function becomes real, leading to an exponential decay of the corresponding signal in $z$.

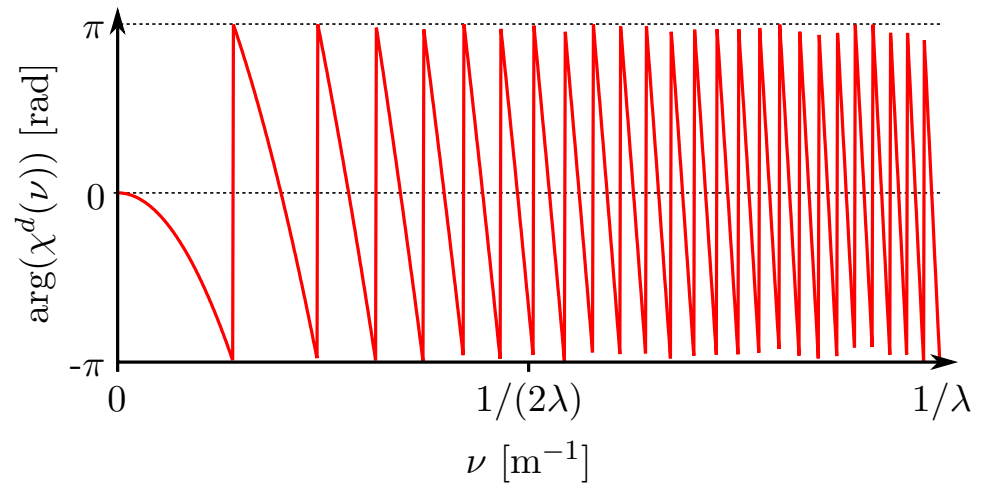

Figure 4.5: Argument of the chirp-function $\chi^{d}(\nu)$ at a distance of $d=2000 \lambda$.

Independently of the precise implementation, in the following we abbreviate the propagation by the operator

$$
\mathcal{D}^{d}[f]: \mathcal{F}^{-1}\left[\chi^{d} \mathcal{F} f\right] .
$$

In this formulation it is easy to see that propagation is associative and commutative, e.g.

$$
\mathcal{D}^{d_{1}+d_{2}} \Psi=\mathcal{D}^{d_{1}} \mathcal{D}^{d_{2}} \Psi=\mathcal{D}^{d_{2}} \mathcal{D}^{d_{1}} \Psi
$$

\footnotetext{
${ }^{3}$ Usually, also the real-space convolution kernel is termed a chirp-function [45]. In this work, to avoid confusion and according to [3] only the Fourier space expression is named like this.

${ }^{4}$ We have excluded "backscattering" by allowing propagation only from the negative to the positive half-space.
} 
and importantly the inverse (as well as the complex conjugated) operator $\mathcal{D}^{-d}$ is given by a propagation about the negative distance, since

$$
\mathcal{D}^{-d} \mathcal{D}^{d}[f]=\mathcal{F}^{-1}[\underbrace{\chi^{-d} \chi^{d}}_{1} \mathcal{F} f]=f .
$$

\subsection{Numerical propagation}

As we have seen in the previous chapter with respect to the Ram-Lak-filter, discretisation can be a non-trivial task - especially when transformations like the FT are involved. This section is dedicated to the numerical implementation of propagation including a close look on occurring artefacts. First, their courses are identified and illustrated. Subsequently, approaches are presented to overcome limitations and artefacts. Finally, examples of propagations in one and two dimensions are presented.

\subsubsection{Discretising for different geometries}

In the following, we assume that the wave field $\Psi$ at the plane $z=0$ is available as a rectangular matrix of equidistant, equisized pixels, as obtained by a numerical projection $^{5}$, for example. Then, as a result of the diffraction formula, the propagated wave field can be calculated in a straightforward manner by considering each pixel as the source of the modified spherical wave in Eq. (4.15). The sum of these waves can be evaluated at any point, which makes this method the most flexible approach for propagation. However, it is not free of artefacts and computationally very expensive if the field has to be evaluated at many points. Numerically more advantageous methods are provided by the convolution formulas in Eq. (4.20) and Eq. (4.25). Note that for a correct implementation of discrete circular convolution, $\Psi$ has to be zero-padded to twice its size and the result has to be cut out appropriately, as explained in chapter 2.2.2. Nevertheless, both formulations allow to obtain the field in a parallel plane at a distance $d$ with high efficiency through a Fast Fourier Transform (FFT). As a drawback, the geometry of the target plane is restricted to that of the input plane, i.e. pixel number, pixel size and the aspect ratio are pre-determined ${ }^{6}$. However, a lateral $x$-y-shift can be introduced, allowing to calculate the propagated field at any region on the target plane. An example is shown in Fig. 4.6. Next to the wave field $\Psi$ in (a), the propagation kernel $g$ in (b) has been sampled at pixels with the same size but twice the pixel number in both directions for correct circular convolution yielding the propagated wave field in (c) with the same pixel geometry as in (a). An arbitrary shift of $g$ as depicted in (d) results in a propagated field (e) shifted about the same distance. As an example object, a (sectorised) zone plate has been chosen because of

\footnotetext{
${ }^{5}$ Different geometries are of course possible but have not been used in this work.

${ }^{6}$ With inserted zero-padding / regridding / interpolation also the pixel-size and geometry can be changed, but this requires an additional operation which is not considered here.
} 


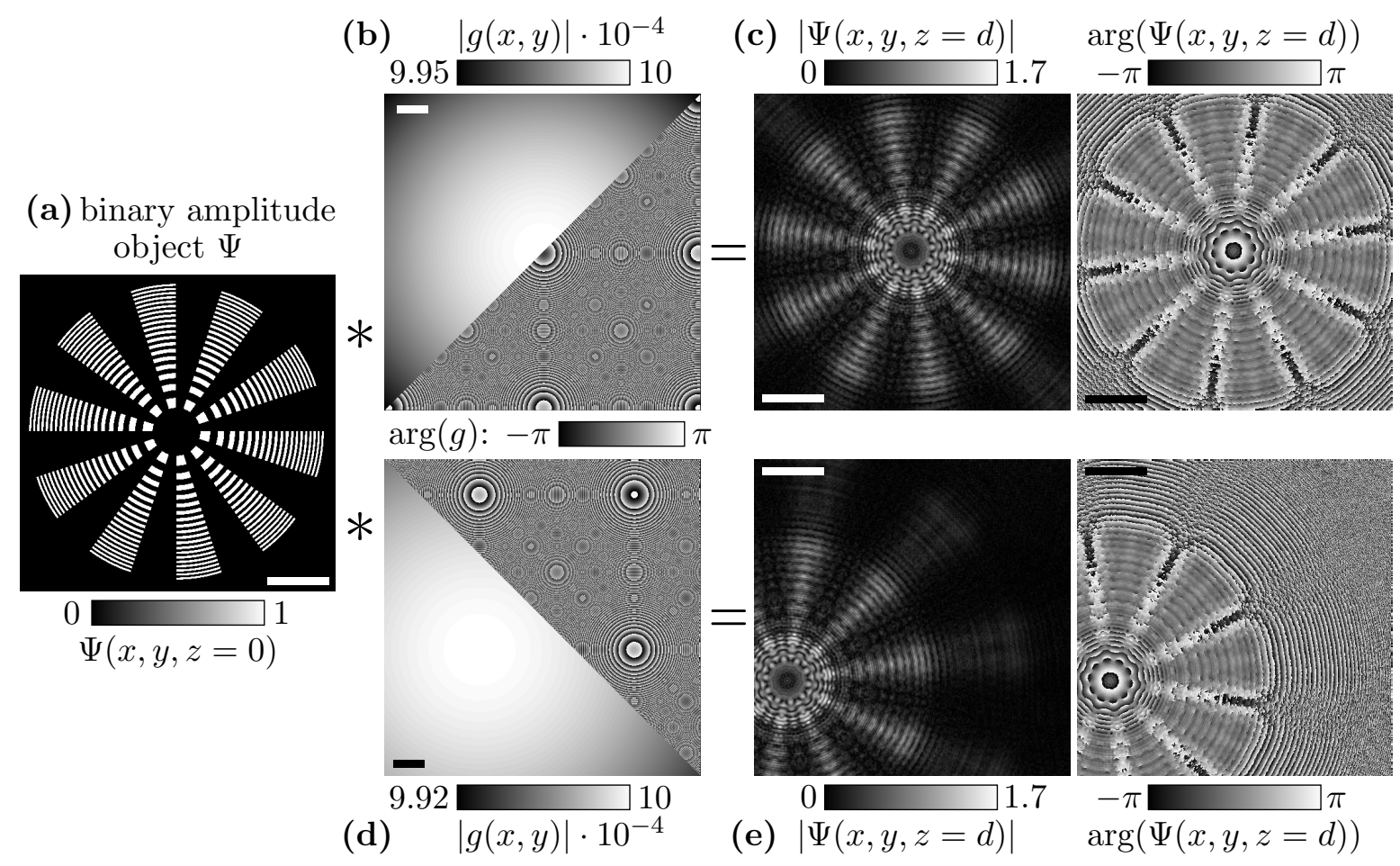

Figure 4.6: Two-dimensional propagation example of a sectorised zone plate. The binary object $\Psi$ with constant phase in (a) is convolved with a propagation kernel $g$ in (b) to obtain the propagated wave field depicted in (c). If the kernel is shifted arbitrarily (d) the propagated field is shifted about the same pixel distance (e) allowing to calculate $\Psi$ anywhere on the distant plane. In this example, $\Psi$ has a size of $512 \times 512 \mathrm{px}^{2}$, and represents a sectorised zone plate with a focal length of $f_{z}=1.5 \cdot 10^{5} \mathrm{px}$. The wavelength is $\lambda=0.1 \mathrm{px}$ and the propagation distance $d=10^{5} \mathrm{px}$. The discrete convolution kernel $g$ has been sampled at $1025 \times 1025 \mathrm{px}^{2}$ with the same pixel size for correct circular convolution. All scale bars represent a width of $100 \mathrm{px}$.

its appealing wave field rich of verifiable features such as different-order focal points in the near field. See appendix 9.3 for details on binary zone plates.

Another example of propagation by convolution is depicted in Fig. 4.7. Here, the " $1+1 \mathrm{~d} " x-z$ field obtained from a $1 \mathrm{~d}$ signal at different distances is calculated, in contrast to the previous example of a $2 \mathrm{~d}$ wave field at a parallel $x-y$ plane. Two methods for this calculation can be utilised as follows: One is the so-called multislice approach. It is carried out by many $1 \mathrm{~d}$ convolutions with their results inscribed in the corresponding column of a $2 \mathrm{~d}$ pixel matrix. If the field has to be evaluated in equidistant steps $\Delta d$, the propagation kernel or Fourier filter $g$ has to be calculated only once. Each new distance can be obtained by the convolution of the last result with $g$ in an incremental manner. For non-equidistant $\Delta d_{i}$, however, $g$ has to be calculated again in every step. An advantage of the multislice approach is the possibility to model material properties like absorption and phase shifts in each iteration. This allows to approximate interaction with matter during propagation through an object exceeding the limits of the projection approximation [47]. 
(a)

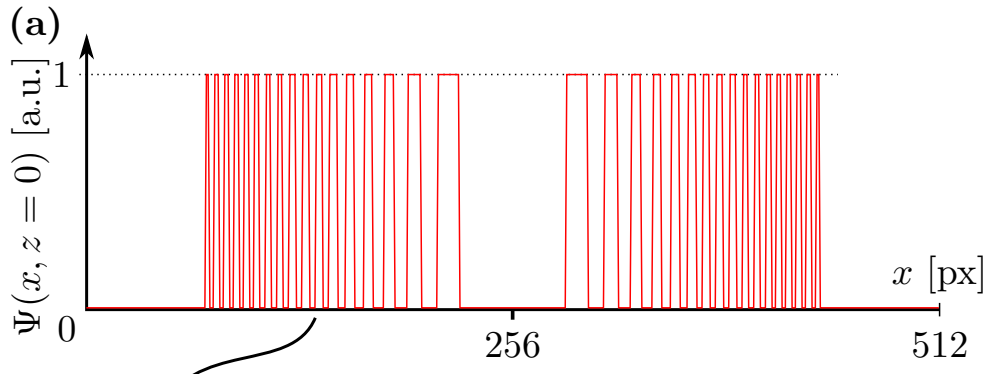

(b)

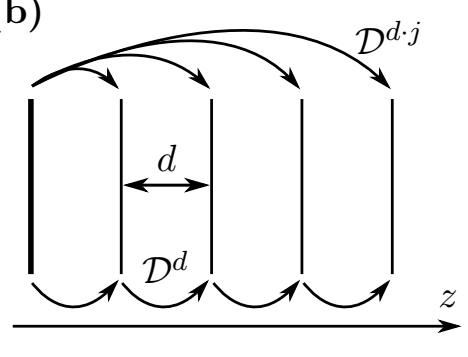

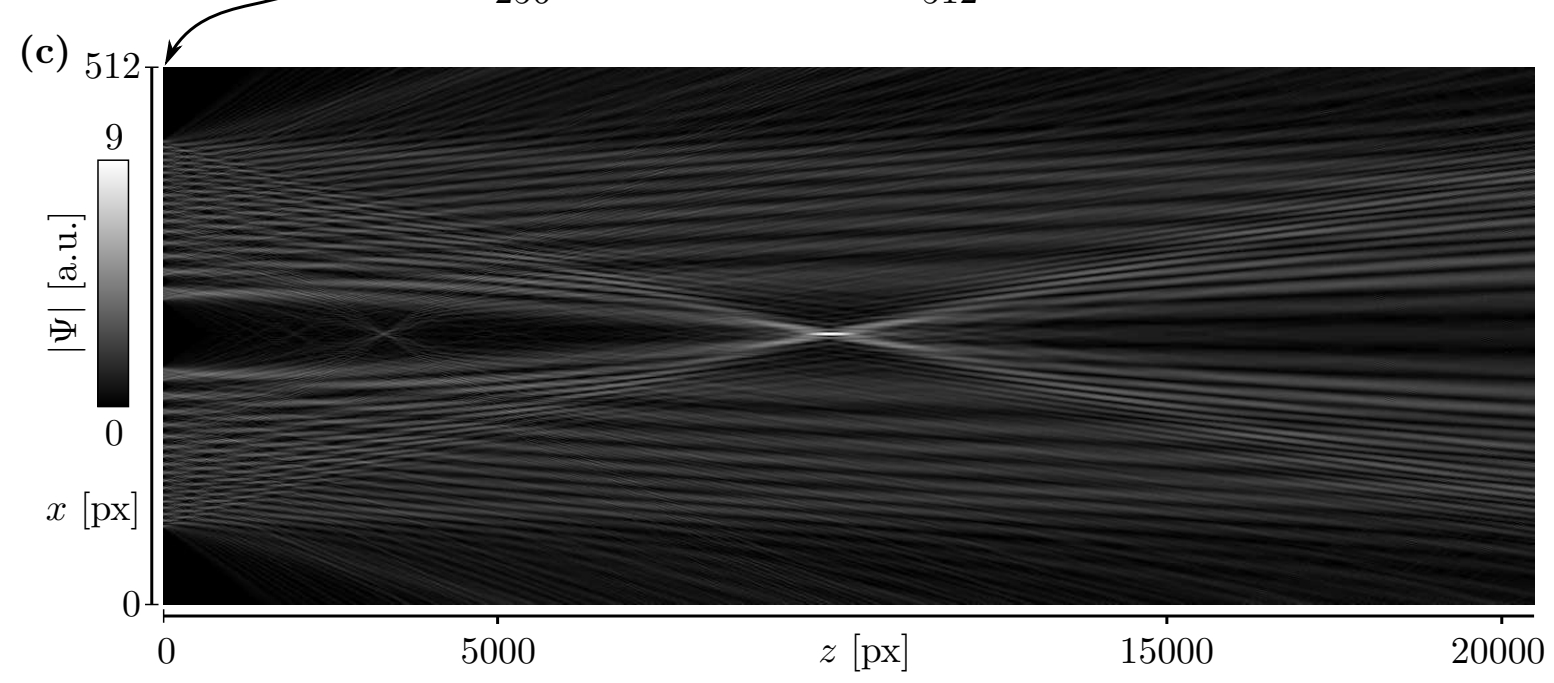

Figure 4.7: Example for multislice propagation. The $512 \mathrm{px}$ binary signal of a $1 \mathrm{~d}$ absorbing zone plate with a focal distance of $z_{z p}=10^{4} \mathrm{px}$ at a wavelength of $\lambda=0.1 \mathrm{px}$ (a) served as initial wave field. For obtaining the propagation along the $z$-axis the initial signal can either be propagated to different distances sequentially or in an incremental manner as sketched in (b). Here, the wave field has been calculated for 1024 planes with a distance of $d=20 \mathrm{px}$ each (c). Propagation has been carried out by the angular-spectrum approach incrementally.

A second method for " $1+1 \mathrm{~d}$ "-propagation is depicted in Fig. 4.8. The initial wave field is inserted into the first column of a $2 \mathrm{~d}$ pixel matrix that equals zero everywhere else. A $2 \mathrm{~d}$ convolution with $g$ sampled for all target distances and lateral shifts yields the same result as the first method. Note that $g$ has the size of the $(x$-)zero-padded input field for circular convolution. Although this technique is not capable of modelling propagation through objects with the same accuracy as the multislice method, it can be used to calculate at least a first Born approximation of wave-matter interaction in a single operation: The object features can simply be inserted arbitrarily in the initial wave field if the padding is extended in $z$ direction. Both methods can be analogously implemented for $3 \mathrm{~d}$ convolution allowing a " $2+1 \mathrm{~d}$ " propagation with the same benefits and restrictions. All approaches can also be formulated in Fourier space but operations like shifts or the equivalent formulation of the kernel in Fig. 4.8 are less intuitive. Further details about the numerical implementation are summarised in appendix 9.2. 


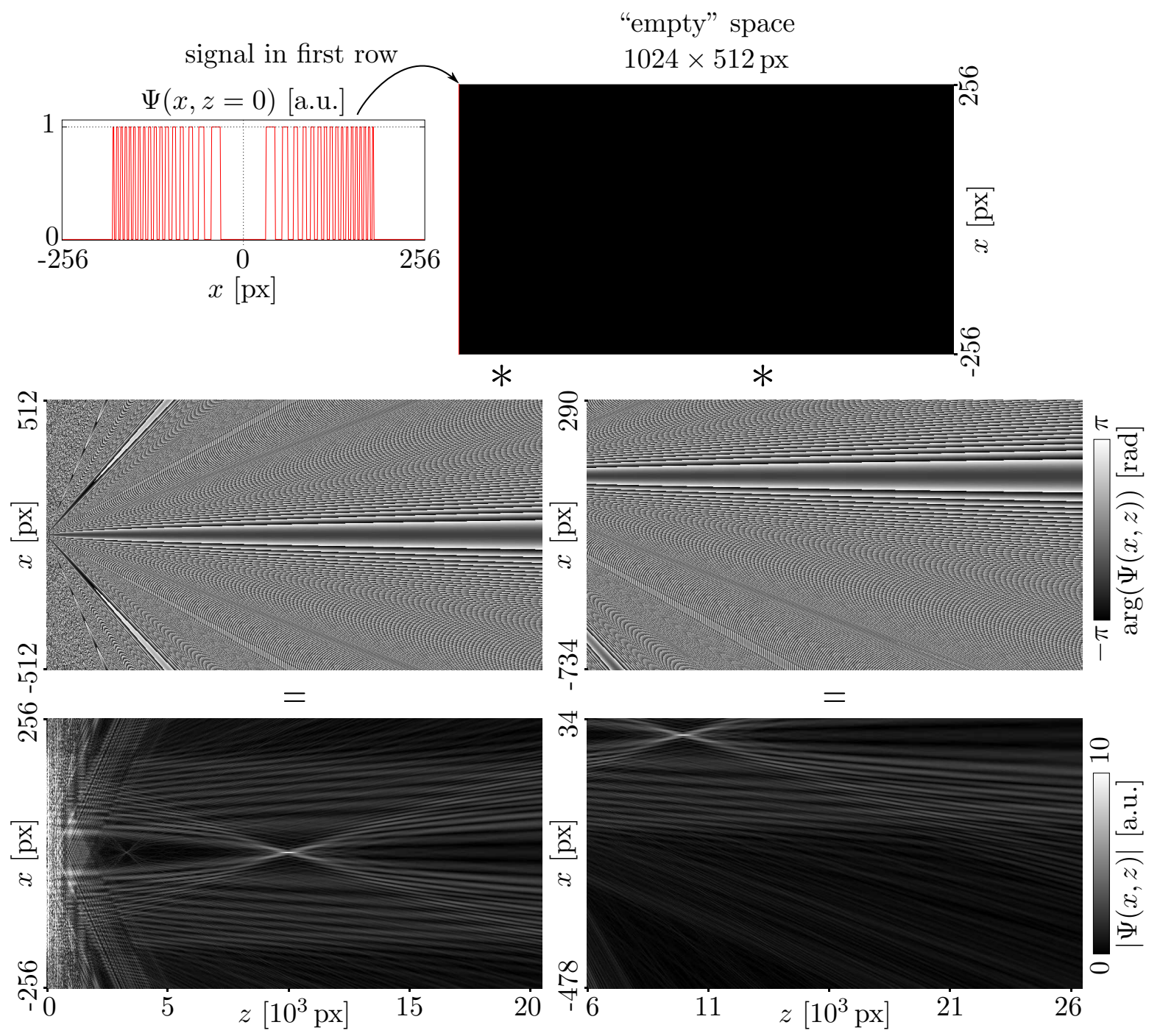

Figure 4.8: Example for $1+1 \mathrm{~d}$ propagation by $2 \mathrm{~d}$ convolution. The initial signal is inserted into the first column of an empty matrix defining the output geometry. A $2 \mathrm{~d}$ convolution with the propagation kernel sampled at all target distances yields the propagated wave field. On the right, an offset of $z_{0}=6000 \mathrm{px}$ and a lateral shift of $x_{0}=-222 \mathrm{px}$ has been applied to the kernel, resulting in a wave field shifted about the same distance. See section 4.3 .2 for a discussion of the artefacts appearing on the left side.

\subsubsection{Considering band limits}

According to Eq. (4.27), the chirp function and the first Sommerfeld diffraction kernel are analytically identical. However, the numerical results reveal eminent differences. As illustrated in Fig. 4.9, the angular-spectrum formulation creates artefacts at large propagation distances while in the convolution approach artefacts predominantly occur at small distances. Both methods allow to find intuitive explanations for their errors. The convolution approach is flawed by aliasing. According to the sampling-theorem it occurs when the signal varies faster than half the sampling frequency. Usually, the 

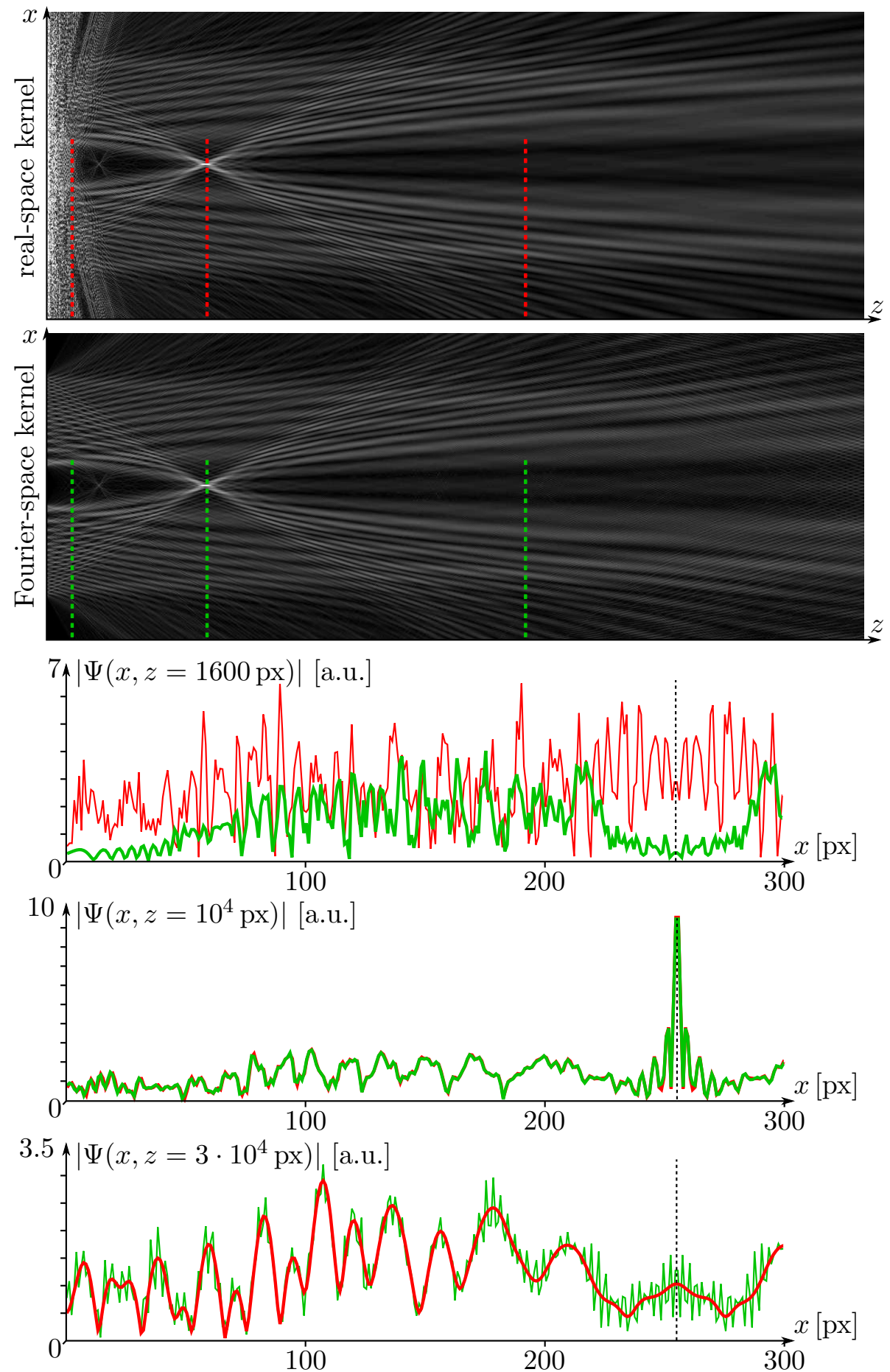

Figure 4.9: Comparison of propagation artefacts. Analytically identical, the sampling of the propagation kernel in real space (top) creates artefacts at short distances while the sampling in Fourier space leads to artefacts at large distances. For both approaches the same conditions have been used, i.e. the angular-spectrum based propagation has been zero-padded to the same size as necessary for circular convolution.

pixels are much larger than the wavelength. Near the origin plane, quickly oscillating waves from many directions interfere. Dramatic variations in the field amplitude within 


\section{Wave field propagation}

the area of a pixel occur which cannot be represented properly. At larger distances from the plane, the $k$-vectors of all contributing waves become more and more parallel. Relative variations on the length scale of a pixel decrease and so do the artefacts. This effect can be well observed at the left kernel in Fig. 4.8, where distinct artefacts occure at the regions of too fast oscillations.

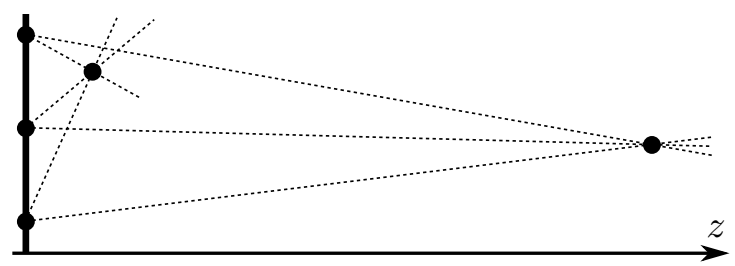

Figure 4.10: For short distances, interfering waves from different directions show fast variations within the sizes of a pixel resulting in aliasing. For larger distances, the $k$ vectors are nearly parallel, yielding only little relative variations.

Using the angular spectrum approach the shape of the artefacts is a result of the periodic repetition inherent to the discrete Fourier transform. The object itself can be considered as infinitely repeated on the input plane. With increasing propagation distance, the signals from the repeated objects spread and interfere with each other. This can be seen particularly well in a (tiled) $1+1 \mathrm{~d}$ propagation as provided in Fig. 4.11: A signal "leaving" the area in lateral direction again enters the region at the opposite border. Consequently, artefacts first occur at the borders of the area, spreading to the whole wave field for increasing propagation distances. Hence, a common approach of artefact

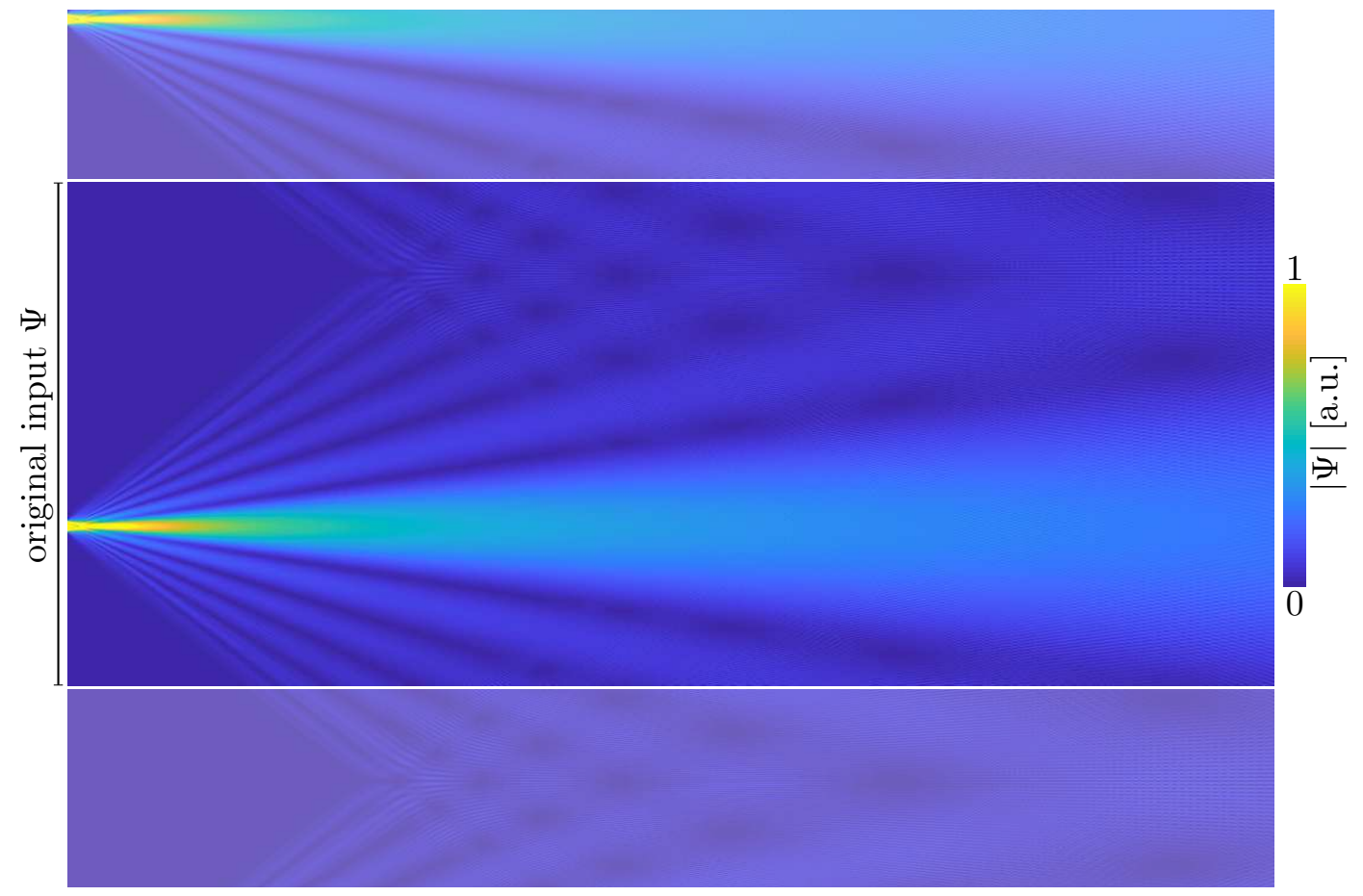

Figure 4.11: The shape of artefacts created by angular-spectrum propagation can be understood by considering periodic repetition immament to the discrete Fourier transform. Spreading signals from repeated objects interfere with the original wave field. 
reduction is to enlarge the distance of the repeated wave fields by zero-padding of the input wave field [45]. The larger the propagation distance, the more padding is required. As we have already seen for the dishing artefacts in tomography, padding only reduces artefacts but does not remove them entirely. A more sophisticated method requires a closer look at the source of the artefacts. Both techniques, the angular-spectrum propagation and the convolution approach, are implemented by a (same-sized) FFT, the only remaining difference is whether the propagation kernel is calculated in real or Fourier space. A common reason for the artefacts and the differences can be identified in aliasing. As shown in $[45,48]$ also angular-spectrum propagation artefacts can be interpreted as aliasing flaws of the chirp function $\chi^{d}=\exp \left(i k d \sqrt{k^{2}-\nu_{x}^{2}-\nu_{y}^{2}}\right)$. For increasing propagation distances, $\chi^{d}$ shows faster oscillations. Beginning at the borders of the sampled Fourier space, they can get faster than the NYQUIST sampling rate which is when the artefacts begin to occur. The solution proposed in [48] is to limit the chirp function to the properly sampled region by an appropriate window function. While this indeed removes the artefacts, certain drawbacks have to be taken into account: While an angular-spectrum-based forward and subsequent backpropagation exactly recovers the original wave field, the effective low-pass filter by windowing the chirp-function ultimately removes information during propagation.

Initially unaware of these findings, a similar formulation of propagation in real space has been developed and used throughout this work. Guided by the question why the angular-spectrum approach produces artefact-free solutions in the near field where the convolution method fails, differences in the angular distribution of the radiation attract attention. In the real-space formulation derived for point sources the signal of a pixel gets distributed to all directions in the positive half-space. Only backpropagation is prohibited by the obliquity-factor. In contrast, the angular spectrum approach limits the maximum angle of radiation. For a pixel size of $\Delta$, the highest frequency oscillation in the discrete Fourier decomposition has a wavelength of $\lambda_{\perp, \min }=2 \Delta$ and we find for the angle

$$
\vartheta_{\max }=\operatorname{asin}\left(\frac{k_{\perp, \max }}{k}\right)=\operatorname{asin}\left(\frac{\lambda}{2 \Delta}\right)
$$

which only depends on the ratio between wavelength $\lambda$ and the pixel size. Only for a sampling size equal to or smaller than the NYQUIST rate $2 \Delta=\lambda$, radiation is propagated to all angles ${ }^{7}$. This observation suggests limiting the convolution kernel to $\vartheta_{\max }$ to take the discrete nature of the pixels into account. For this purpose, many window functions are thinkable. Empirically, good results have been achieved by modifying the obliquity factor $\cos \vartheta$ to

$$
O F:= \begin{cases}\cos \left(\frac{\pi}{2} \frac{\vartheta}{\vartheta_{\max }}\right) & \text { for } \vartheta<\vartheta_{\text {max }} \\ 0 & \text { else }\end{cases}
$$

approaching the original factor for NYQUIST sampling $\vartheta_{\max }=\pi / 2$. This effectively limits the real-space convolution kernel to the region where its phase oscillaton is sam-

\footnotetext{
7 Sampling smaller than the NYQUIST distance requires angles of radiation larger than $90^{\circ}$. As mentioned before, this leads to evanescent waves in the angular spectrum.
} 
pled adequately. Comparisons as provided in Fig. 4.12 reveal excellent reproductions of the propagated wave field with a complete removal of the artefacts - independent from the propagation distance. A numerical implementation can be found in the appendix 9.2 with comments on technical details like the treatment of even and odd input pixel numbers.
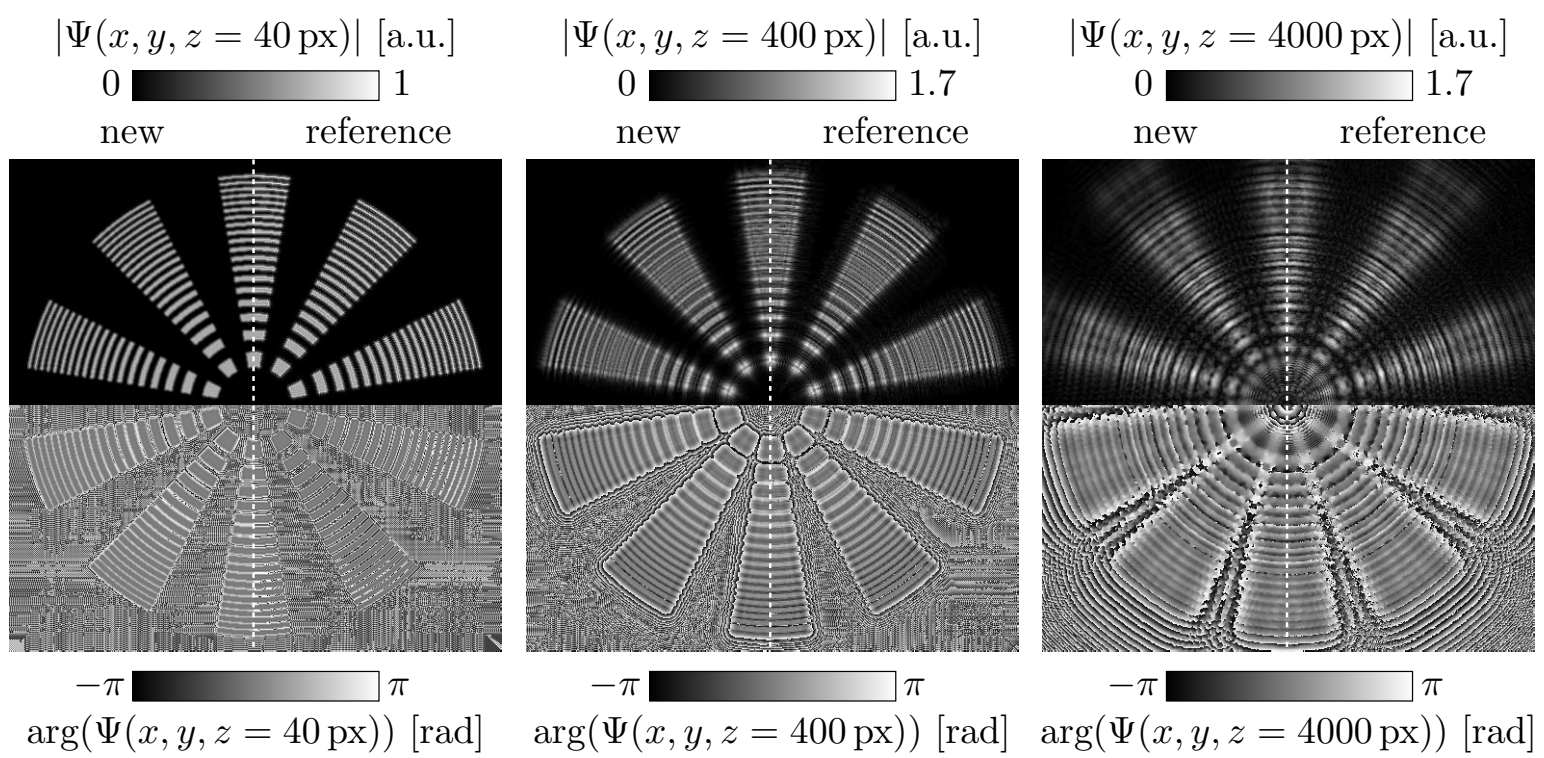

Figure 4.12: 2d propagation examples with limited obliquity factor. At short distances $z$ where the wave field is utterly flawed by conventional convolution propagation, limiting the angle of radiation clearly removes the artefacts introduced by aliasing. The comparison with reference simulations obtained by angular-spectrum propagation with large padding reveals excellent reproductions of the wave field.

We close this section with a remark about perfect sampling. The maximum angle of radiation still hitting a detector with diameter $L=N \cdot \Delta$ at a distance $d_{p}$ is given by

$$
\vartheta_{\max }^{\prime}=\operatorname{atan}\left(\frac{L}{2 d_{p}}\right) .
$$

If $\vartheta_{\max }$ exeeds this angle, parts of the wave field are not captured by the detector. On the other hand, if $\vartheta_{\max }<\vartheta_{\text {max }}^{\prime}$, the detector is not illuminated completely. Thus, with $\sin (\operatorname{atan}(x))=x / \sqrt{1+x^{2}}$, setting $\vartheta_{\text {max }}^{\prime}=\vartheta_{\max }$ from Eq. (4.31) yields

$$
\Delta=\frac{\lambda}{L} \sqrt{d_{p}^{2}+L^{2} / 4}
$$

The same expression for $\Delta$ has been derived in [45] strictly from the NYQUIST-SHANNON sampling criterion for the chirp function in Fourier space. It can be shown that for a pixel size $\Delta$, the sampling is ideal and matches the analytical propagation kernel. The chirp function does not suffer from aliasing and the wave field is not oversampled. Consequently, angular spectrum and convolution based sampling are equal. However, adjusting this situation is often not possible since it may require too large pixels for 

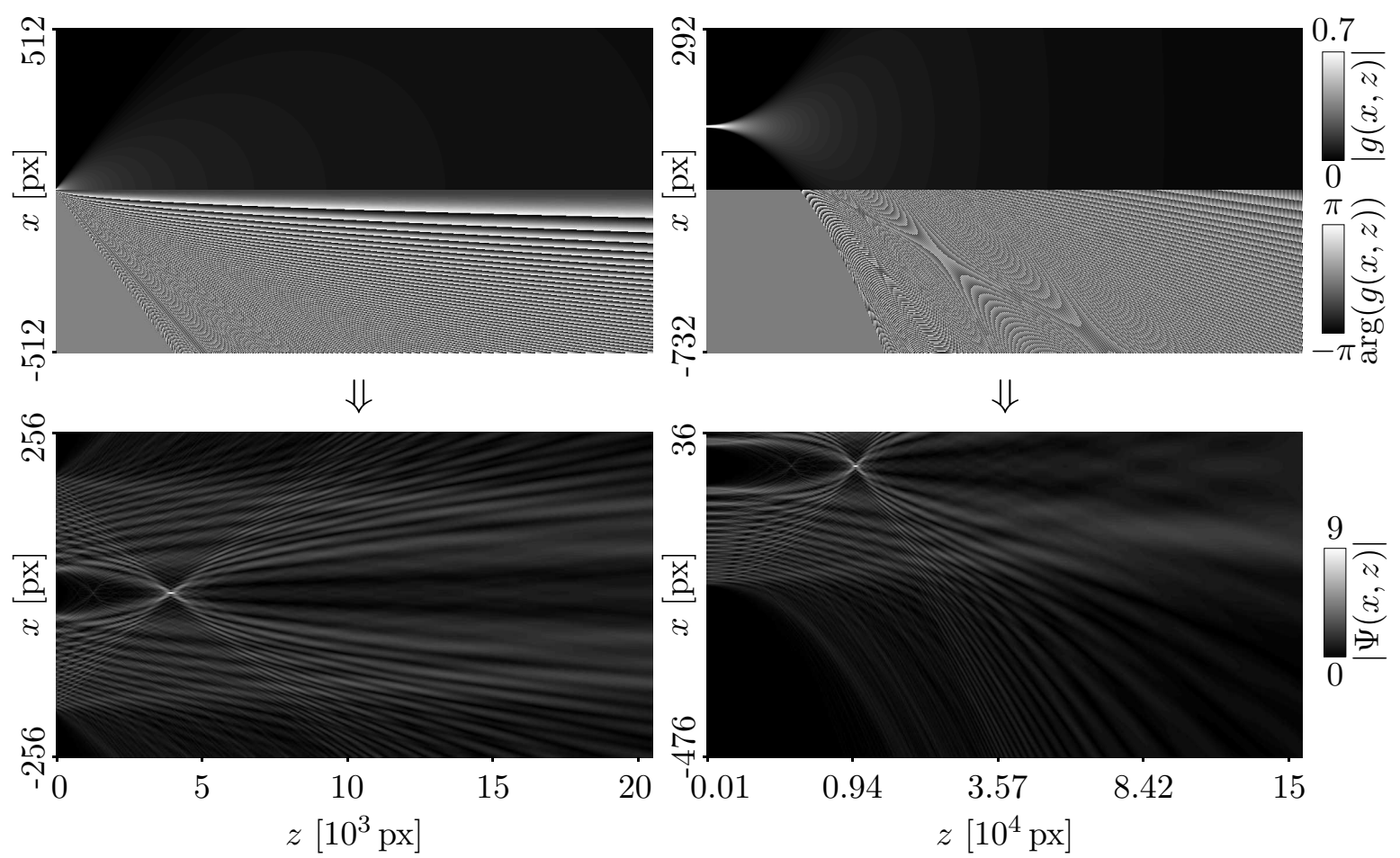

Figure 4.13: $1+1 \mathrm{~d}$ propagation with modified obliquity factor. The top row shows modulus and phase of the convolution kernel in combined pictures, the bottom row depicts the convolution result with the zone plate signal introduced before. The limited obliquity factor clearly removes the artefacts shown in Fig. 4.9 (left side). The right side depicts a more complex situation where the kernel shows both a vertical offset and an exponential increase of the propagation distance. Hence, both the near field and the slowly developing field at larger distances are visualised in good detail.

adequate sampling of $\Psi$ or too many pixels for a practical calculation. Alternatively, the expression resolved to

$$
d_{p}=L \sqrt{\frac{\Delta^{2}}{\lambda^{2}}-\frac{1}{4}}
$$

gives an estimation of which method applies in which region:

for $d \ll d_{p}$ : angular-spectrum approach accurate

for $d \gg d_{p}$ : convolution approach accurate.

Hence, simply switching the propagation methods can also be exploited to reduce artefacts considerably. The main advantage of limiting the obliquity factor is the conceptual simplicity. The intuitive and consistent model yields good results at all distances. It enables to implement non-trivial propagation like those shown in Fig. 4.13 in a straightforward manner and easily allows to estimate how and where the signal from a given source pixel contributes to the result. 


\subsection{D propagation}

In the case of a weakly interacting object, Eq. (3.15) already showed that the exit wave for unit amplitude plane wave illumination is given by

$$
\Psi(x, y, z=0)=\exp \left(-i k \mathcal{P}\left[\delta_{\omega}(\vec{r})-i \beta_{\omega}(\vec{r})\right]\right) .
$$

If the integrated absorption $k \mathcal{P} \beta_{\omega}$ and phase shifts $k \mathcal{P} \delta_{\omega}$ remain small too, the exponential function can be linearised to

$$
\Psi(x, y, z=0) \approx 1-i k \mathcal{P} \delta_{\omega}(\vec{r})-k \mathcal{P} \beta_{\omega}(\vec{r})
$$

With this, a subsequent propagation acts directly on the projections and the wave field at the distance $z=d$ can be expressed as

$$
\Psi(x, y, z=d) \approx \exp (i k d)-i k \mathcal{D}_{d} \mathcal{P} \delta_{\omega}(\vec{r})-k \mathcal{D}_{d} \mathcal{P} \beta_{\omega}(\vec{r})
$$

This offers the possibility to change the order of the projection and propagation operation. With $f(\vec{r})$ a placeholder for either $\delta_{\omega}(\vec{r})$ or $\beta_{\omega}(\vec{r})$, we can write

$$
\begin{aligned}
\mathcal{D}_{2 d} \mathcal{P} f= & \mathcal{F}_{2 d}^{-1}\left[\chi^{d} \cdot \mathcal{F}_{2 d} \mathcal{P} f\right] \\
= & \frac{1}{4 \pi^{2}} \iint_{-\infty}^{\infty} \underbrace{\exp \left(i d \sqrt{k^{2}-\nu_{x}^{2}-\nu_{y}^{2}}\right)}_{\chi^{d}} \\
& \cdot[\underbrace{\left[\int_{-\infty}^{\infty}\left(\int_{-\infty}^{\infty} f \mathrm{~d} z\right) \exp \left(-i\left(\nu_{x} x+\nu_{y} y\right)\right) \mathrm{d} x \mathrm{~d} y\right]}_{\mathcal{F}_{2 d} \mathcal{P} f} \exp \left(i\left(\nu_{x} x+\nu_{y} y\right)\right) \mathrm{d} \nu_{x} \mathrm{~d} \nu_{y} \\
= & \frac{1}{4 \pi^{2}} \int_{-\infty}^{\infty} \int_{-\infty}^{\infty} \delta_{D}\left(\nu_{z}\right) \cdot \exp \left(i d \sqrt{k^{2}-\nu_{x}^{2}-\nu_{y}^{2}-\nu_{z}^{2}}\right) \\
& \cdot\left[\iint_{-\infty}^{\infty} f \exp \left(-i\left(\nu_{x} x+\nu_{y} y+\nu_{z} z\right)\right) \mathrm{d} x \mathrm{~d} y \mathrm{~d} z\right] \mathrm{d} \nu_{z} \exp \left(i\left(\nu_{x} x+\nu_{y} y\right)\right) \mathrm{d} \nu_{x} \mathrm{~d} \nu_{y}
\end{aligned}
$$

In the second step, the Fourier slice theorem has been used. The projection is written as a central slice of the $3 \mathrm{~d}$ Fourier transform of $f$, selected by the DIRAC delta distribution $\delta_{D}$. Note that $\delta_{D}$ does not only act on $\hat{f}$ but also on the chirp function $\chi^{d}$, which has been extended to three dimensions in such a way that all of its central slices are identical. Figuratively speaking, all central slices of $\hat{f}$ get multiplied by the same propagation function and thus all possible projections become propagated about the 
same distance. In the next step, $\delta_{d}$ is expressed as the inverse Fourier transform of a one, yielding

$$
\begin{aligned}
\mathcal{D}_{2 d} \mathcal{P} f= & \frac{1}{8 \pi^{3}} \int_{-\infty}^{\infty} \iint_{-\infty}^{\infty} \exp \left(i d \sqrt{k^{2}-\nu_{x}^{2}-\nu_{y}^{2}-\nu_{z}^{2}}\right) \\
& \cdot \mathcal{F}_{3 d}[f] \exp \left(i\left(\nu_{x} x+\nu_{y} y+\nu_{z} z\right)\right) \mathrm{d} \nu_{x} \mathrm{~d} \nu_{y} \mathrm{~d} \nu_{z} \mathrm{~d} z \\
= & \mathcal{P F}_{3 d}^{-1}\left[\chi_{3 d}^{d} \cdot \mathcal{F}_{3 d} f\right] \\
= & \mathcal{P D}_{3 d} f .
\end{aligned}
$$

This result clearly holds for all orientations of $f$. Thus, as sketched in Fig 4.14, instead of propagating a $(2 \mathrm{~d})$ projection, one can also project from a $3 \mathrm{~d}$ propagated object $\mathcal{D}_{3 d} f(\vec{r})$, obtained by a multiplication with a generalised $3 \mathrm{~d}$ chirp function

$$
\chi_{3 d}^{d}:=\exp \left(i d \sqrt{k^{2}-\nu_{x}^{2}-\nu_{y}^{2}-\nu_{z}^{2}}\right)
$$

in Fourier space. Clearly, this $3 d$ propagation can be formulated equivalently as a convolution in real space and the numerical considerations from the previous section can be applied as well.

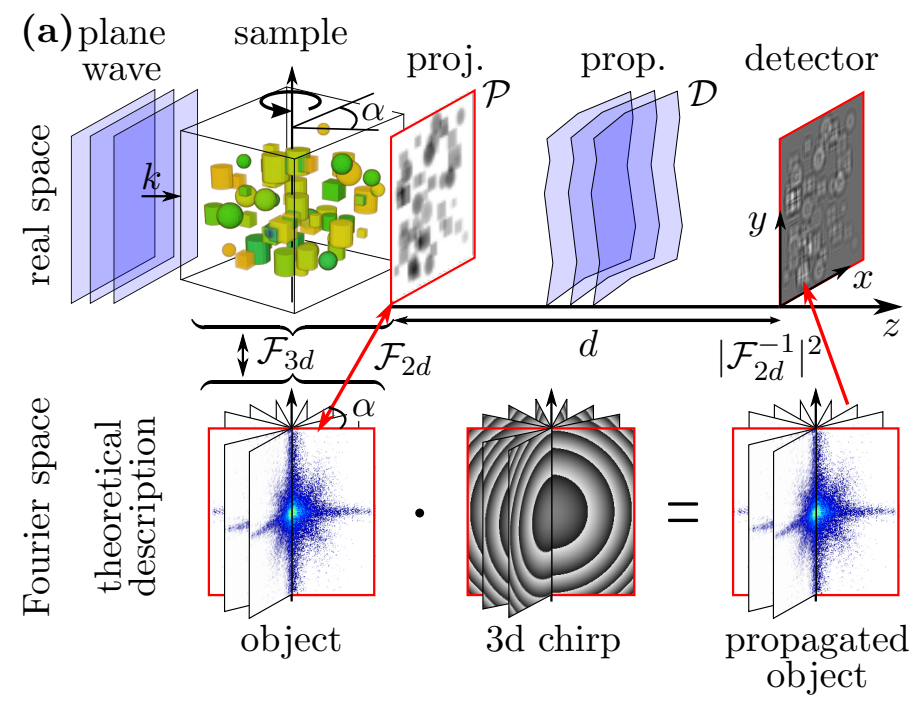

(b)

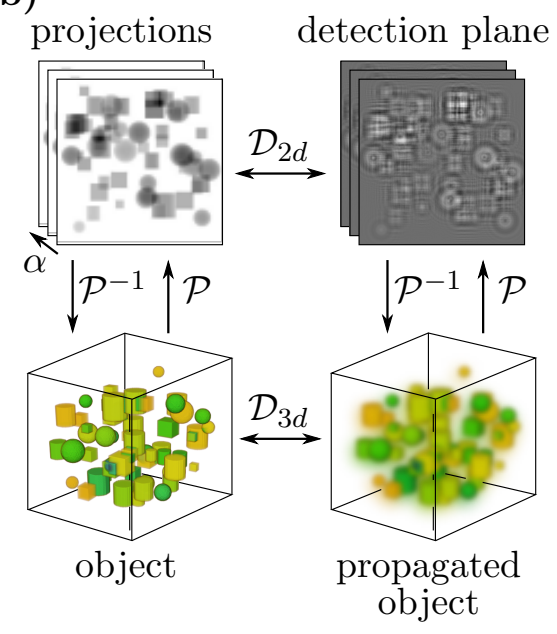

Figure 4.14: Idea of $3 \mathrm{~d}$ propagation: (a) A plane wave illuminating a weakly interacting object results in an exit wave in the $x-y$ plane as given by the projection approximation followed by free-space propagation to the distant detector. This process can be described in Fourier space by multiplying the appropriate central slices of the object's FT with the radially symmetric propagation chirp function. The propagation of all possible central slices is identical to the multiplication of the FT of the object with a generalised $3 \mathrm{~d}$ chirp function, yielding a $3 \mathrm{~d}$ propagated object. With a projection from this object, the propagated wave field can be obtained. Hence, projection $\mathcal{P}$ and propagation operation $\mathcal{D}$ can be permuted for weakly interacting objects (b). Image obtained from [49]. 
The propagated object itself has no real physical representation but many beneficial properties. Importantly, it proves that propagated projections are still consistent in terms of tomography, since they can be considered as projections of this mathematical object. Hence, alignment procedures, tomographic reconstructions as well as sinogram retrieval based on consistency can be applied directly to propagated projections of weakly interacting objects. In addition, the propagated object can be reprojected to arbitrary directions yielding e.g. edge enhanced projections ${ }^{8}$ in experimentally inaccessible directions. As presented in the next chapter, the concept of $3 \mathrm{~d}$ propagation is particularly useful in simulations and phase-retrieval algorithms. It not only summarises many $2 \mathrm{~d}$ propagations to a single and still very efficient $3 \mathrm{~d}$ operation, but also allows to implement previously impossible boundary conditions.
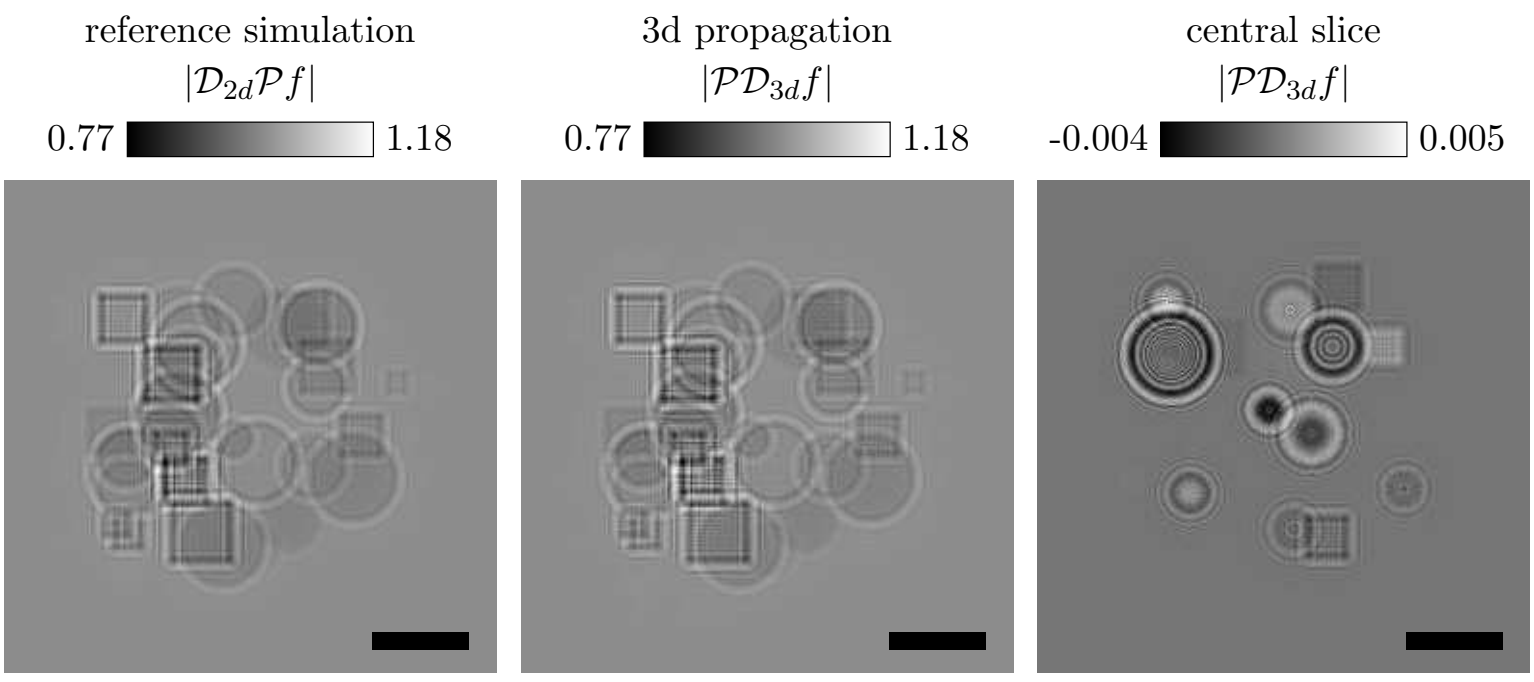

Figure 4.15: The comparison of a conventional wave field simulation (first project then propagate) (a) with the $3 \mathrm{~d}$ propagation (first propagate then project) (b) shows an excellent agreement. (c) depicts the central $x-z$ slice of the propagated object. With $\lambda=0.01 \mathrm{px}$ all scale bars denote a size of $50 \mathrm{px}$ corresponding to 5000 wavelengths. The object contains 20 spheres and 15 cubes with variable sizes and complex optical indices, randomly arranged in a $256^{3}$ voxel grid.

\subsection{The Fresnel-Scaling Theorem}

Up to this point, we have assumed a parallel beam illumination for projection and propagation. However, many real setups use a diverging beam, better described by a point source. In tomography, divergence can be incorporated by appropriate fan-beam or cone-beam backprojection algorithms based on similar principles as introduced in the previous chapter $[25,27]$. A small divergence can be safely neglected if the maximum beam inclination remains smaller than one pixel per diameter of the volume. In case of

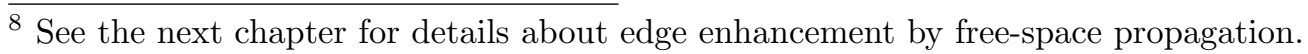


a cubic $N^{3}$ volume, the maximum angle then has to be limited by $\vartheta_{\max }<\operatorname{atan}(1 / N)$. For $N$ in the order of a few thousand, angles in the order of a millirad are acceptable. But even for such small angles, the divergence cannot be neglected during propagation about distances of several metres to ensure proper magnification and phase contrast. Fortunately, as shown in e.g. [3], the results of parallel beam propagation can still be used, but have to be expressed in terms of effective parameters.
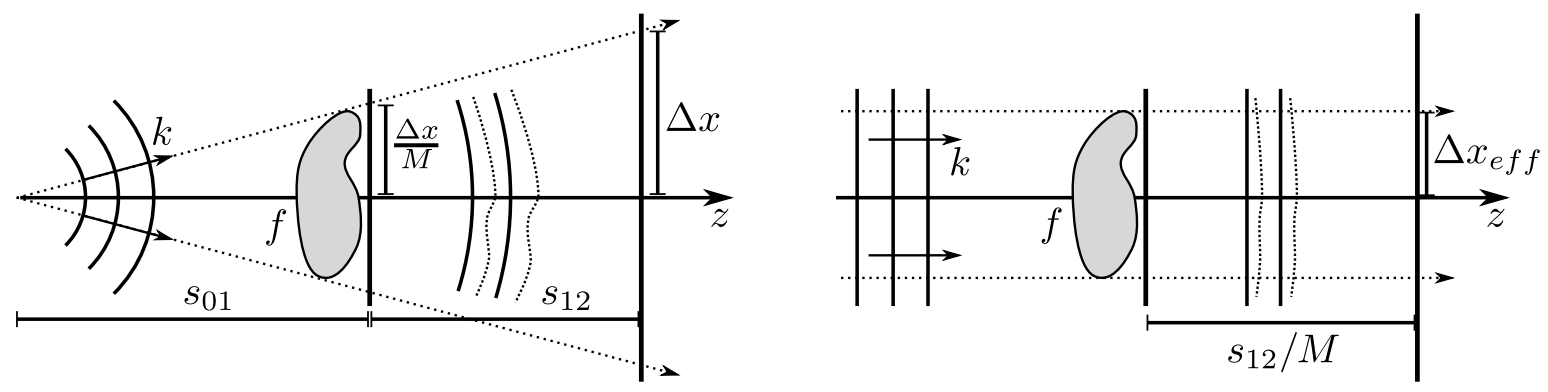

Figure 4.16: Fresnel-Scaling-Theorem: The illumination with a divergent spherical wave can be expressed by an effective parallel beam geometry.

From geometrical observations as sketched in Fig. 4.16, an object at distance $s_{01}$ behind a point source appears magnified by a factor

$$
M=\frac{s_{02}}{s_{01}}
$$

on a plane at $s_{02}=s_{01}+s_{12}$. The effective detector size at the object's position is then given by

$$
\Delta x_{e f f}=\frac{\Delta x}{M}
$$

compared to the real detector size $\Delta x$ at $s_{02}$. In a paraxial approximation of propagation and the illuminating wave front, the intensity $I \propto|\Psi|^{2}$ at distance $s_{02}$ can be identified as [3]

$$
I\left(x, y, s_{12}\right)=\frac{1}{M^{2}} I_{\|}\left(\frac{x}{M}, \frac{y}{M}, \frac{s_{12}}{M}\right),
$$

provided the projection approximation holds. Hence, the intensity at the real propagation distance $s_{12}$ in a divergent wave field can be expressed as a scaled version of the intensity $I_{\|}$obtained by parallel beam propagation at an effective propagation distance $s_{12} / M$. 



\section{Propagation-based phase-contrast imaging}

To date, most applications of X-ray imaging including clinical radiography and $\mathrm{CT}$, are based on absorption contrast alone. However, especially for hard X-rays the absorption of radiation is very small in soft matter like biological tissue. Obtaining projections with a good signal-to-noise ratio would either require a high intensity of the illumination possibly destroying the sample or long exposure times, which are not compatible with the goal of fast image acquisition for dynamic tomography. Thus, we need to enhance the contrast by utilising the much stronger phase shifting properties of the sample with $\delta_{\omega}$ up to three orders of magnitude larger than $\beta_{\omega}$ in the complex index of diffraction $n_{\omega}=1-\delta_{\omega}+i \beta_{\omega}$. In practice, phase contrast makes the measurement of small biological samples possible in the first place. Among the many different approaches to visualise phase shifts, only a few are suitable for time-resolved imaging. For example, many phase contrast techniques require lateral or longitudinal scanning of the object in the probing beam, notably in ptychography [50], Talbot interferometry [51], edgeillumination $[52,53]$ or speckle-based phase contrast techniques [54]. Therefore, we chose phase contrast by free propagation $[8,9,55]$ based on the self-interference of the wave front behind the object. The phase shift information of a projection is thereby converted into measurable brightness variations. A single recording without scanning is sufficient and no additional optical elements are inserted in the optical path behind the sample, such as in Zernike based phase contrast [56], assuring a high signal and dose efficiency.

The method was introduced by DenNis GaBOR in an exciting paper published in 1948 [57], for which he eventually was awarded with the Nobel Prize in 1971. It is called (in-line) holography from ancient Greek ŏגos (holos) meaning "whole" and $\gamma \rho \alpha \dot{\varphi} \varphi \iota \nu$ (graphein) for "to write" or "to record" as in tomography. The basic idea is to record the interference pattern of the object signal with a reference wave, encoding not only the absorption but also the relative phase shifts in brightness variations. Thus, coherence as provided by synchrotron sources in case of X-rays is a requirement for the method. For weakly interacting objects the illumination itself can be considered as the reference wave, being superposed by the weak object signal yielding distinct interference patterns on a distant detector. Notably, holography implies near-field imaging, in contrast to coherent diffraction imaging (CDI) where the detection takes place in the optical far field described by the Fourier transform of the exit wave. While the experimental setup is similar and in both cases requires no optical components between object and detector, holography has particular advantages: It is more dose efficient with respect to the image quality obtainable from a given dose of radiation and more robust in terms of partial coherence $[58,59]$, which is helpful for fast imaging. Moreover, tomography requires a careful alignment of the setup and of the object in the axis of rotation. In holographic imaging, these steps can be carried out in a straight-forward manner compared to the Fourier geometry of CDI, even when the details of the object cannot be seen on the detector but only the rough shape of the sample. 
The main drawback of both techniques is that retrieving the phase information encoded in the intensity recordings is a non-trivial task. Additional constraints or information about the sample are required since still only a fraction of the wave field's information is captured. Note that both amplitude and phase of the wave field are to be reconstructed from only a single intensity measurement. But even in this context holography allows to guess the dimensions and features of the object more easily directly from the projections, valuable for the determination of support or sparsity information as well as for predictions about the material composition.

The important task of phase retrieval is the main topic of the present chapter, i.e. phase contrast is not only used to enhance the detector signal but a quantitative reconstruction of the wave field is the ultimate goal. In the previous chapter wave propagation has been considered mainly in mathematical and technical terms. Here, we will focus on its physical properties. Many wave-optical phenomena, notably the interference patterns behind slits, can be well understood by the diffraction integral with its instructive basis of elementary waves. But also the angular spectrum approach vividly reveals many fundamental wave-optical effects. Here, we will focus on the latter approach, offering a more intuitive insight in phenomena like contrast formation, the main topic of the first section of this chapter: By following the evolution of the wave field's intensity in free space, first we will investigate which kind of information about the object can be obtained from different regions of the wave field. In the second part, mathematical methods are presented to retrieve the information encoded in the holograms and compensate (at least partially) for lost information by including a priori knowledge. To this end, one-step methods based on Fourier filters as well as iterative phase-retrieval procedures are introduced. Addressing the main challenge of phase retrieval, i.e. the provision and proper application of prior knowledge, the chapter closes with presenting approaches to combine phase retrieval and tomographic reconstruction using (tomographic) consistency as a constraint for phase retrieval. To this end, the first reconstructions of experimental data are shown to illustrate the value of consistency in iterative holographic phase retrieval.

\subsection{Information content of a wave field}

To capture all information transported by a wave field, its amplitude and phase have to be acquired over an infinite plane ${ }^{1}$ at a sampling resolution smaller than the wavelength of the radiation. Clearly, this is not possible with any available detector system. Instead, only the intensity of the wave field is measured at a limited field of view with a comparatively large pixel size. Therefore, as we will see in this section, the degree of information attainable depends on the geometry of the experiment, i.e. varying quantities can be measured at different positions of the wave field.

\footnotetext{
1 Alternatively, the wave field can be acquired for all angles of radiation, but then, as we have seen in the previous chapter, also the normal derivative has to be measured.
} 


\subsubsection{The diffraction limit}

For numerical propagation, limiting the angle of radiation by introducing a modified obliquity factor as demonstrated in the previous chapter may look arbitrary from a physical point of view. However, it is based on a physical principle, namely the long known resolution limit of optical systems. As a reminder, the NYQUIST-SHANNON sampling criterion states that equidistant sample values within a distance of $\lambda_{m i n} / 2$ are sufficient to exactly reconstruct the signal up to a minimum wavelength (highest frequency) of $\lambda_{\text {min }}$. Vice versa, a band-limited signal with a minimum frequency of $\lambda_{\min }$ can at least reproduce features with the size $\lambda_{\min } / 2$, although even smaller but low intensity features can appear in the signal, a phenomenon known as super-oscillations [60].

Let the smallest feature in the $x$-direction of a wave field have a size of $d_{\text {min }}$. Then, the necessary minimum wavelength for proper sampling is $\lambda_{\perp \text { min }} \approx 2 d_{\text {min }}$. According to Eq. (4.23), the corresponding plane wave with photon wavelength $\lambda$ leaves the plane at an angle of

$$
\vartheta_{\max }=\operatorname{asin}\left(\frac{\lambda}{\lambda_{\perp \min }}\right) \approx \operatorname{asin}\left(\frac{\lambda}{2 d_{\min }}\right) .
$$

Resolved for $d_{\text {min }}$, one obtains

$$
d_{\text {min }} \approx \frac{\lambda}{2 \sin \vartheta}
$$

This expression is known as the resolution limit, stating that no structures smaller than $d_{\min }$ can be safely resolved if the wave field has been captured up to a maximum angle of $\vartheta$. Often, the sine in Eq. (5.2) is complemented by the index of refraction $n$ of the propagation medium, summarised as the numerical aperture $N A:=n \sin \vartheta$. Depending on the definition of when exactly two diffraction limited points can be considered as distinguishable, Eq. (5.2) can be found with different pre-factors in the literature. Consequently, limiting the angle of radiation in numerical propagation restricts the result to the physically reasonable region. Moreover, the equation shows that the resolution and thus the information obtainable from an object is practically limited by the angular acceptance of the detector. In case of X-rays, the wavelength imposing a fundamental resolution limit is sufficiently small.

\subsubsection{Imaging regimes}

The angular spectrum, i.e. the connection between object feature size and angle of radiation, provides insight into the evolution of the wave field on its way downstream the sample. Except for evanescent waves practically vanishing after a propagation distance of a few wavelengths, the modulus of the wave fields' spectrum remains constant - independent of the propagation distance $z$. The unit-amplitude chirp function only multiplies the spectrum by a phase factor, shifting the oscillations in the $x-y$ planes relative to each other. The smaller the oscillation wavelength $\lambda_{\perp}$, the larger is the angle of radiation of the corresponding wave and the higher is the relative shift during propagation. 


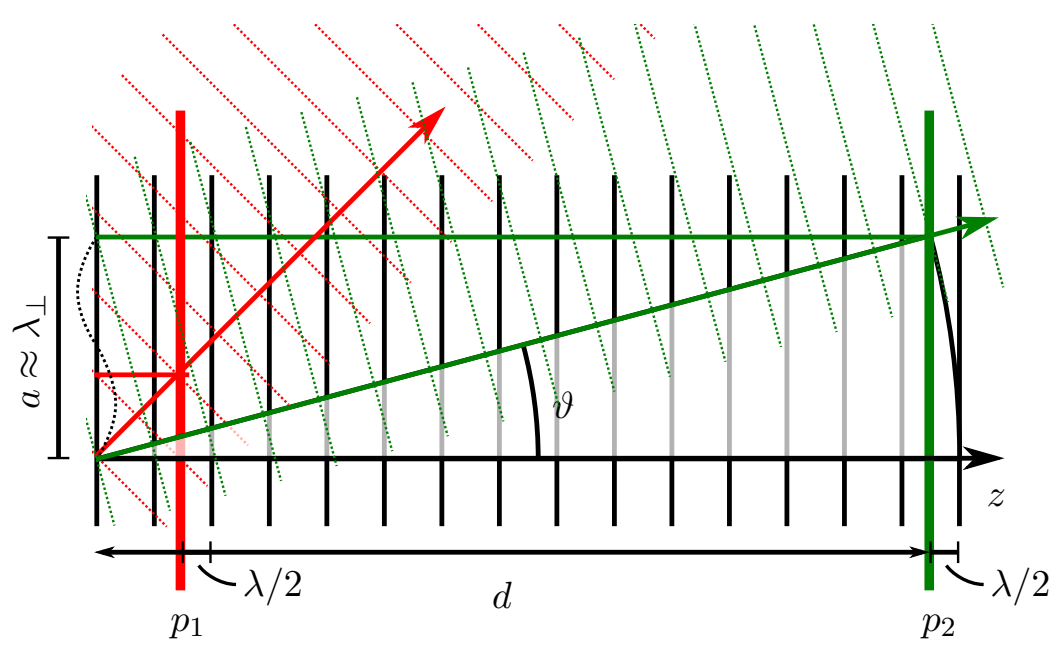

Figure 5.1: The smaller the wavelength $\lambda_{\perp}$ in the plane perpendicular to the incident radiation, the larger is the corresponding angle of radiation $\vartheta$ - and the shorter is the distance $z$ on the optical axis the wave shows a relative phase shift of $\pi$ with respect to the primary undeflected wave. For the red-coloured wave, this relative phase shift is reached at the red plane $p_{1}$, while the green-coloured wave with a smaller angle $\vartheta$ needs all the distance to the plane $p_{2}$ for the the same relative phase shift.

Both situations are sketched in Fig. 5.1. Along the $z$-direction, the red-coloured wave has a relative phase shift of $\pi$ with respect to the non-deflected "primary" wave at the red plane $p_{1}$. The green-coloured wave with a smaller angle $\vartheta$ needs all the distance to the plane $p_{2}$ for the same relative phase shift of $\pi$. Hence, the small oscillations are expected to be the first to show propagation effects. Indeed, this is known as edgeenhancement, emphasizing the sharp edges in the projections for short propagation distances. This can be seen in the examples depicted in Fig. 5.2 for small propagation distances. With higher distances, larger oscillations get shifted significantly, too, leading to spreading and blurring of the edges. Finally, at very large distances, no structure can be recognised at all. Only the amount of energy transported by the waves to the different directions is of importance, approaching the Fourier transform of the original function. A relative phaseshift of $\pi$ compared to the non-deflected wave is reached at the distance

$$
\begin{aligned}
d k & \stackrel{!}{=} d k \cos (\vartheta)+\pi \\
\Rightarrow d & =\frac{\lambda}{2(1-\cos \vartheta)}=\frac{\lambda}{2\left(1-\sqrt{1-\lambda^{2} / \lambda_{\perp}^{2}}\right)} \\
& \approx \frac{\lambda}{2\left(1+\frac{\lambda^{2}}{2 \lambda_{\perp}^{2}}-1\right)}=\frac{\lambda_{\perp}^{2}}{\lambda},
\end{aligned}
$$


since usually $\lambda \ll \lambda_{\perp}$. Alternatively, as indicated in Fig. 5.1 (b), the situation can be interpreted as a lateral shift of the wave about its own lateral wavelength $\lambda_{\perp}$. With $\tan (\operatorname{asin}(x))=x / \sqrt{1-x^{2}}$ the corresponding distance is found to be

$$
\vartheta=\operatorname{asin}\left(\frac{\lambda}{\lambda_{\perp}}\right)=\operatorname{atan}\left(\frac{\lambda_{\perp}}{d}\right) \Rightarrow d=\frac{\lambda_{\perp}^{2}}{\lambda} \sqrt{1-\lambda^{2} / \lambda_{\perp}^{2}}
$$

If $a=\lambda_{\perp}$ is chosen as the characteristic size of the wave field with $a \gg \lambda$, the square root vanishes, yielding

$$
1=\frac{a^{2}}{d \lambda}=: F_{a}
$$

$F_{a}$ is known as the Fresnel number, a measure for the imaging regime. The "characteristic oscillation" shifted laterally about its own wavelength $\left(F_{a} \approx 1\right)$ (or equivalently, having reached a relative phase shift about $\pi$ on the $z$-axis with respect to the undeflected wave) defines the so-called holographic regime with distinct interference between all modes in the order of $a$. As can be seen in Fig. 5.2, only low-frequency patterns like the background in the top-left phase quadrant remain invisible. For $F_{a} \gg 1$, the characteristic oscillation is shifted much less than one wavelength in the propagated wave field and no interference effects appear at all. Only the amplitude variations are visible in the intensity patterns. This so-called contact regime widely used in radiology can be described properly by geometrical optics. Decreasing Fresnel numbers allow to first see the edges of the phase-content (very weak at $F_{10}=100$, clear at $F_{10}=10$ in Fig. 5.2). In contrast, the characteristic oscillation shifted far more than a period $(F \ll 1)$ defines the far field or Fraunhofer regime. The original structure is not recognisable anymore. In this case, for large distances $d \gg \lambda$ and small objects, the obliquity factor and all quadratic terms $\propto r^{-2}$ can be neglected in the diffraction integral Eq. (4.15), resulting in

$$
\Psi\left(x_{0}, y_{0}, z=d\right)=\frac{1}{i \lambda} \int_{-\infty}^{\infty} \Psi(x, y, z=0) \frac{\exp \left(i k\left|\vec{r}-\vec{r}_{0}\right|\right)}{\left|\vec{r}-\vec{r}_{0}\right|} \mathrm{d} x \mathrm{~d} y
$$

The denominator $\left|\vec{r}-\vec{r}_{0}\right| \approx d$ can be approximated by the propagation distance, while in the argument of the oscillating exponential function, the second order of the series expansion

$$
\begin{aligned}
\left|\vec{r}-\vec{r}_{0}\right| & =\sqrt{\left(x-x_{0}\right)^{2}+\left(y-y_{0}\right)^{2}+d^{2}} \\
& \approx d+\frac{\left(x-x_{0}\right)^{2}+\left(y-y_{0}\right)^{2}}{2 d} \\
& =d+\frac{x^{2}+y^{2}}{2 d}+\frac{x_{0}^{2}+y_{0}^{2}}{2 d}-\frac{x x_{0}+y y_{0}}{d}
\end{aligned}
$$

is also taken into account, resulting in

$$
\Psi\left(x_{0}, y_{0}, z=d\right)=\frac{\exp (i k d)}{i \lambda d} \exp \left(\frac{i k}{2 d}\left(x_{0}^{2}+y_{0}^{2}\right)\right) \times
$$




\section{Propagation-based phase-contrast imaging}
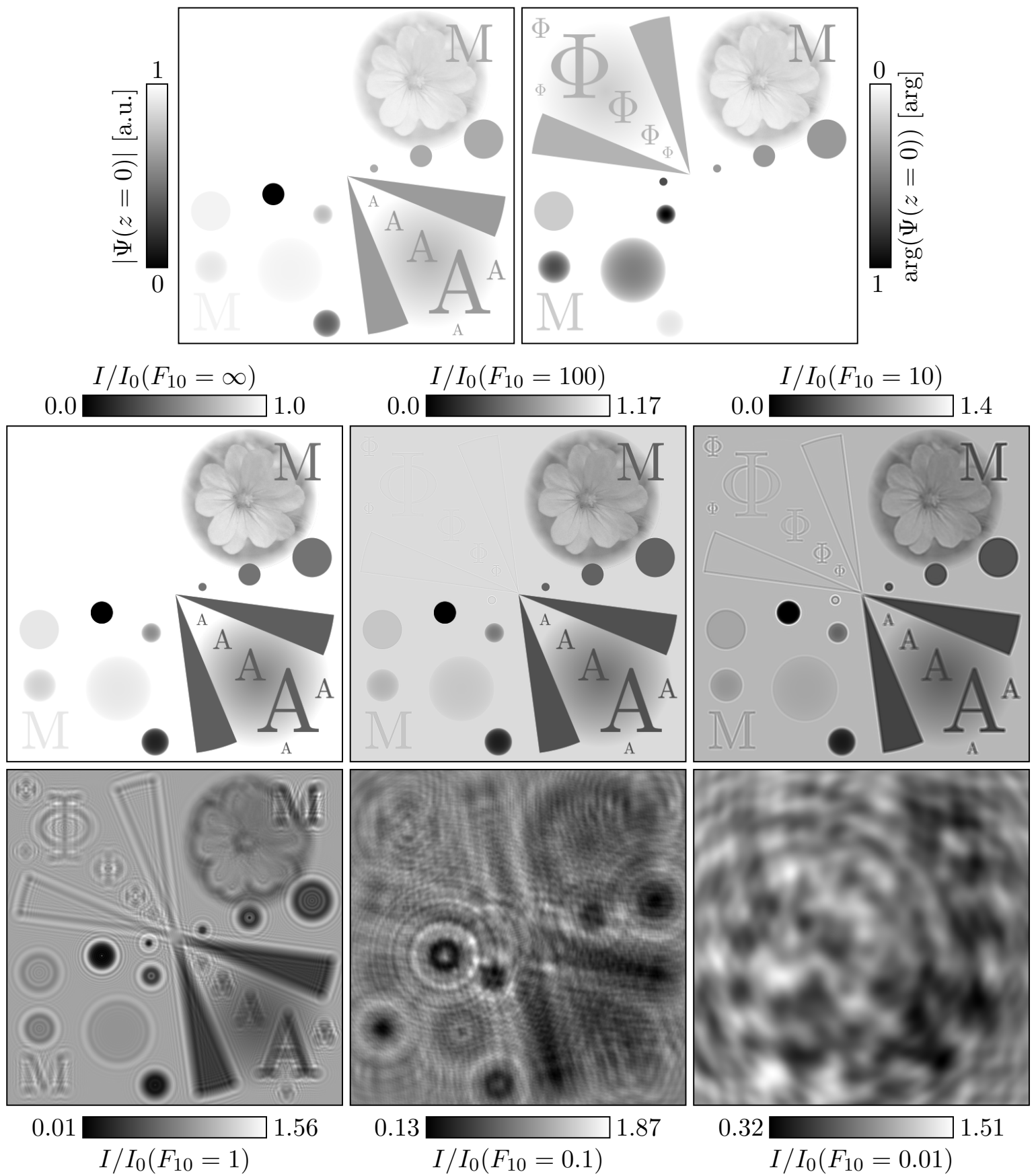

Figure 5.2: Simulated intensity patterns for different Fresnel numbers. The top row shows amplitude and phase of an exit wave containing different structures. The top left quadrant contains a pure phase variation, marked by a $\Phi$ of different sizes, whereas the bottom right quadrant only persists of purely absorbing features marked by an A. The two remaining quadrants are marked with an $\mathrm{M}$ for a mixed composition, e.g. circles, projected spheres and an image of a flower representing a natural signal form, all with varying $\beta / \delta$ ratios. All images have a side length of $512 \mathrm{px}$. The wavelength has been chosen to $\lambda=0.01 \mathrm{px}$ and the characteristic size to $a=10 \mathrm{px}$, slightly less than the diameter of the smallest circle. 


$$
\iint_{-\infty}^{\infty} \Psi(x, y, z=0) \underbrace{\exp \left(\frac{i k}{2 d}\left(x^{2}+y^{2}\right)\right)}_{\exp \left(i \pi \frac{x^{2}+y^{2}}{\lambda d}\right)} \exp \left(-\frac{i k}{d}\left(x x_{0}+y y_{0}\right)\right) \mathrm{d} x \mathrm{~d} y .
$$

The argument of the underbraced function is similar to the Fresnel number $a^{2} / \lambda d \ll 1$, which is small in the given regime. Thus, the term can be replaced by 1 for all $x, y$ where $\Psi$ contributes considerably. With $\tilde{k}_{x}=k x_{0} / d$ and $\tilde{k}_{y}=k y_{0} / d$, Eq. (5.8) can be written as the Fourier transform of the function $\Psi$ in the plane $z=0$ :

$$
\begin{aligned}
\Psi\left(x_{0}, y_{0}, z=d\right)= & \frac{\exp (i k d)}{i \lambda d} \exp \left(\frac{i k}{2 d}\left(x_{0}^{2}+y_{0}^{2}\right)\right) \times \\
& \int_{-\infty}^{\infty} \int_{\infty}^{\infty} \Psi(x, y, z=0) \exp \left(-i\left(\tilde{k}_{x} x+\tilde{k}_{y} y\right)\right) \mathrm{d} x \mathrm{~d} y .
\end{aligned}
$$

Notably, for the same wavelength $\lambda$ and the same distance $d$, differently sized features $a$ of the object may exhibit different properties. The propagated signal of very small objects can show far-field characteristics while the interference pattern of larger objects is limited to the near field.

\subsubsection{Contrast transfer}

For a more quantitative insight, we need to consider that common detectors measure the intensity, defined as the time-averaged power input per (pixel) area, up to a remarkable single-photon energy resolution[3, 61]. As mentioned before, this quantity

$$
I(x, y, z) \propto|\Psi(x, y, z)|^{2}
$$

is proportional to the modulus of the wave field squared. Hence, only the wave's amplitude $|\Psi|$ is measured and all information about its phase has vanished. However, this does not mean that all information about the object's phase shifts is lost. As we will see in more detail later, the initial phase shifts during free-space propagation crucially contribute to the measurable intensity patterns. At the detector distance $d$ behind an object, these are given $b$

$$
\begin{aligned}
I(x, y, z=d) & =\left|\mathcal{D}^{d} \Psi_{s}(x, y, z=0)\right|^{2} \\
& =\left(\mathcal{D}^{d} \Psi_{s}\right)\left(\mathcal{D}^{d} \Psi_{s}\right)^{*} \\
& =\left(\mathcal{D}^{d} \Psi_{s}\right)\left(\mathcal{D}^{-d} \Psi_{s}^{*}\right),
\end{aligned}
$$

which is simply the propagated wave field multiplied with the complex conjugated wave $\Psi_{s}^{*}$ propagated about the negative distance - which practically 'flips' the wave front. This leads to an alternative interpretation of the so-called phase problem, which can be 


\section{Propagation-based phase-contrast imaging}

seen more clearly in an approximation of weakly interacting objects. If the argument $\Phi$ of the exit wave $\Psi_{s}=\Psi_{0} \exp (\Phi)$ remains sufficiently small, the linearisiation

$$
\Psi_{s}(x, y, z=0) \approx \Psi_{0} \cdot(1+\Phi(x, y, z))
$$

allows to express the intensity by

$$
\begin{aligned}
\frac{I(x, y, z=d)}{\left|\Psi_{0}\right|^{2}} & \approx \mathcal{D}^{d}[1+\Phi] \cdot \mathcal{D}^{-d}\left[1+\Phi^{*}\right] \\
& =\left(e^{i k d}+\mathcal{D}^{d} \Phi\right)\left(e^{-i k d}+\mathcal{D}^{-d} \Phi^{*}\right) \\
& =1+e^{i k d} \underbrace{\mathcal{D}^{-d} \Phi^{*}}_{\text {twin }}+e^{-i k d} \underbrace{\mathcal{D}^{d} \Phi}_{\text {image }}+\underbrace{\left|\mathcal{D}^{d} \Phi\right|^{2}}_{\ll 1} .
\end{aligned}
$$

The quadratic term can be safely neglected. In this approximation, the intensity is expressed as the propagated image $\Phi$ superposed by its so-called twin image, which is the complex conjugated image propagated about the negative distance.

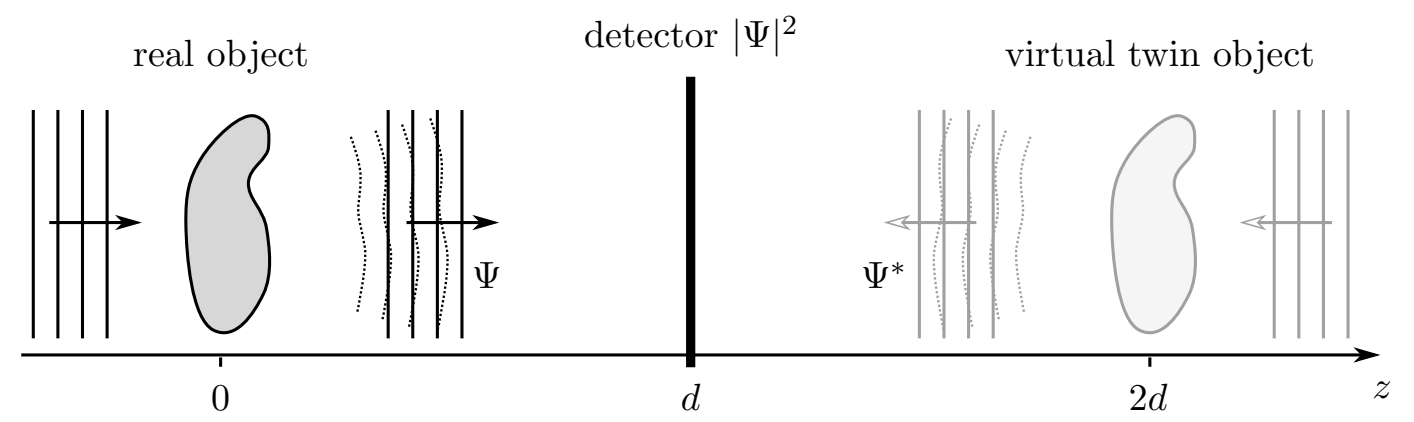

Figure 5.3: The intensity pattern at $z=d$ can be interpreted as the original wave superposed by a wave originating from a complex conjugated twin object at $z=2 d$ and travelling in the inverse direction.

In Fourier space, the propagation operations can be expressed more suitably by a multiplication with the chirp function $\chi^{d}$, yielding

$$
\mathcal{F}\left[\frac{I(x, y, z=d)}{\left|\Psi_{0}\right|^{2}}\right] \approx 2 \pi \delta_{D}+e^{i k d} \chi^{-d} \mathcal{F}\left[\Phi^{*}\right]+e^{-i k d} \chi^{d} \mathcal{F}[\Phi]
$$

with the DIRAC delta distribution $\delta_{D}$. We recall that the projection approximation of an object described by the index of refraction $n=1-\delta_{\omega}+i \beta_{\omega}$ and illuminated by a plane wave with amplitude $\Psi_{0}$ (see Eq. (3.15)) is given by

$$
\Psi_{s}(x, y, z=0)=\Psi_{0} \exp \underbrace{\left(-i k \mathcal{P}\left[\delta_{\omega}(x, y, z)-i \beta_{\omega}(x, y, z)\right]\right)}_{=: \Phi} .
$$

Inserting in Eq. (5.14), provided that absorption and phase shifts are sufficiently small, the separation of $\bar{\delta}:=\mathcal{F} \mathcal{P} \delta_{\omega}$ from $\bar{\beta}:=\mathcal{F} \mathcal{P} \beta_{\omega}$ yields

$$
\mathcal{F}\left[\frac{I(x, y, z=d)}{\left|\Psi_{0}\right|^{2}}\right] \approx 2 \pi \delta_{D}+e^{i k d} \chi^{-d}(i k \bar{\delta}-k \bar{\beta})-e^{-i k d} \chi^{d}(i k \bar{\delta}+k \bar{\beta})
$$




$$
=2 \pi \delta_{D}+i k \bar{\delta} \underbrace{\left(e^{i k d} \chi^{-d}-e^{-i k d} \chi^{d}\right)}_{\xi-\xi^{*}=2 i \Im(\xi)}-k \bar{\beta} \underbrace{\left(e^{i k d} \chi^{-d}+e^{-i k d} \chi^{d}\right)}_{\xi+\xi^{*}=2 \Re(\xi)} .
$$

The symmetrical phase function

$$
\begin{aligned}
\xi\left(\nu_{\perp}\right) & =e^{i k d} \chi^{-d}=\exp \left[i d\left(k-\sqrt{k^{2}-\nu_{\perp}^{2}}\right)\right] \\
& =\exp \left[i k d\left(1-\sqrt{1-\nu_{\perp}^{2} / k^{2}}\right)\right] \\
& \approx \exp (\underbrace{i d \frac{\nu_{\perp}^{2}}{2 k}}_{i \varphi})
\end{aligned}
$$

introduced in the last step has been approximated using $\sqrt{1-x^{2}} \approx 1-x^{2} / 2$ since $\nu_{\perp} / k \ll 1$. The real and imaginary part can be written explicitely as sine and cosine of $\varphi$, leading to

$$
\mathcal{F}\left[\frac{I(x, y, z=d)}{\left|\Psi_{0}\right|^{2}}\right] \approx 2 \pi \delta_{D}-2 k \bar{\delta} \sin (\varphi)-2 k \bar{\beta} \cos (\varphi) .
$$

In this form, the equation is known as the Contrast Transfer Function (CTF) describing the contributions of the object's amplitude $\bar{\beta}$ and phase $\bar{\delta}$ information to the intensity patterns [62-64]. The graph of phase-CTF $(\mathrm{pCTF}, \sin (\varphi))$ and absorption-CTF $(\mathrm{aCTF}, \cos (\varphi))$ is plotted in Fig. 5.4 for the reduced spatial frequency $\sqrt{\lambda z}\left|\nu_{\perp}\right|$. It reaffirms the qualitative consideration from the previous section but allows to describe the information contained within the intensity patterns in a quantitative way:

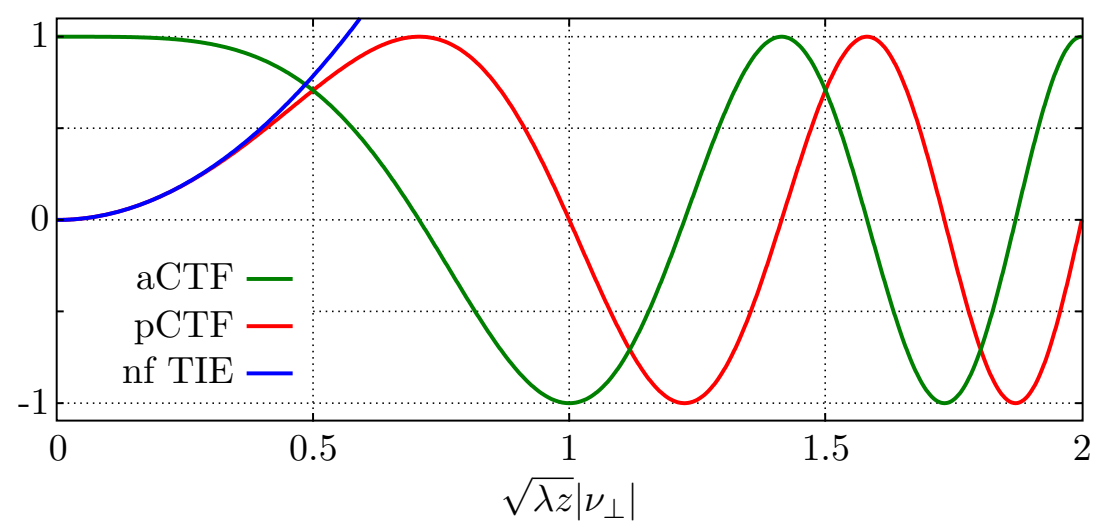

Figure 5.4: Shape of the contrast transfer function in reduced units. Note that the $\mathrm{pCTF}$ as well as its approximation by the near-field transport-of-intensity equation (nf TIE) are very small for low spatial frequencies.

In the contact regime $(\varphi \ll 1)$, the intensity pattern is predominantly defined by absorption since the pCTF is practically zero. For increasing distances, phase information becomes visible in intensity patterns as the pCTF rises. At first, its shape can be well approximated by a parabola $\propto \nu_{\perp}^{2}$ as indicated in Fig. 5.4. Since the multiplication with a parabola in Fourier space is the equivalent of a second derivative in real space, 
the curvature of the phase front defines the contribution of $\bar{\delta}$. In particular, the edges of the projected phase shifts are enhanced in this so-called direct contrast regime while the low-frequency components are still not present in the images. This situation can be described alternatively by a near-field approximation of the transport of intensity equation (TIE) [3] and is sometimes called the TIE regime. At even larger distances, in the holographic regime, phase contrast transfer affects all frequency ranges until up the 'deep' holographic regime where the original structure of the sample is barely recognisable. Low-frequency components of the initial phase shifts become present in the images, too, with the exception of the origin that always equals zero. Moreover, some higher frequencies are lost due to the zero-crossings of the CTF. They appear as rings in the Fourier transform of the intensity images, also known as power spectral density, as illustrated in Fig. 5.5.

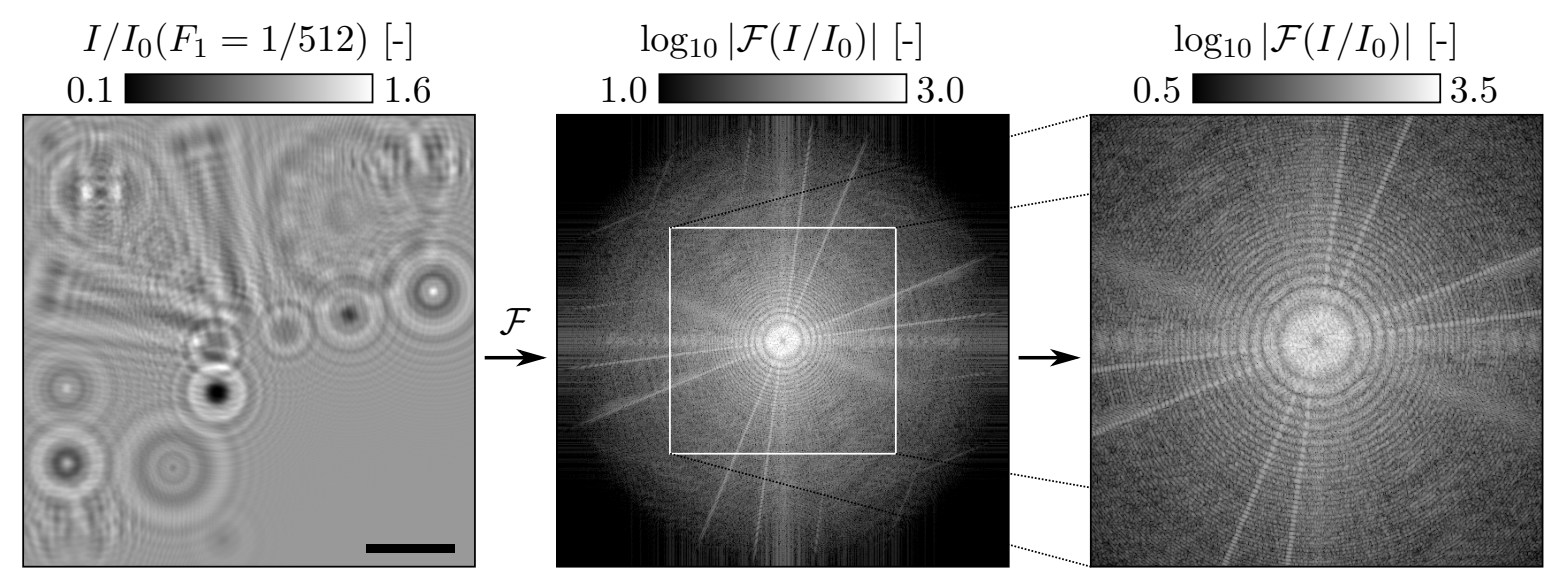

Figure 5.5: The lost spatial frequencies due to zeros in the pCTF can be well observed as rings in the Fourier transform of a hologram (power spectral density, PSD) obtained from a pure phase object (as depicted in Fig. 5.2 but with the constant amplitude of one.). The scale bar indicates a width of $100 \mathrm{px}$.

\subsubsection{Numerical imaging constraints}

We close this section with some considerations about the ideal imaging regime with respect to numerical propagation. In the paraxial approximation, propagation can be described completely in terms of the Fresnel number $F_{a}$, i.e. only the ratio of the characteristic size $a$ to the wavelength $\lambda$ and the distance $d$ is of importance. As a characteristic size, it is convenient to choose the pixel size $a=\Delta$ including only the geometry of the experiment independent from the concrete sample. Hence, for this choice, we have

$$
F_{1}=\frac{\Delta^{2}}{\lambda d}
$$


If $d=L \sqrt{\Delta^{2} / \lambda^{2}-1 / 4}$ is chosen as the ideal sampling distance of the chirp function for a detector with diameter $L$ as derived in Eq. (4.35), we obtain

$$
F_{1}=\frac{\Delta}{L \sqrt{1-\frac{\lambda^{2}}{4 \Delta^{2}}}} .
$$

In the basis of this equation, we again find the resolution limit, since the numerical aperture precisely corresponds to the pixel size as the resolution element. The (limited) cone of radiation emanating from each pixel increases to the same diameter as the detector in the corresponding distance, hence, even from the object pixels at the border, at least a quarter of the symmetrical signal (the complete radius) is captured. : With the wavelength $\lambda \ll \Delta$ usually being much smaller than the pixel size, the Fresnel number for ideal sampling can be well approximated by

$$
F_{1} \approx \frac{\Delta}{L}=\frac{1}{N_{\Delta}}
$$

the inverse of the number of diagonal detector pixels $N_{\Delta}$. Since this number is on the order of $10^{3}$, ideal sampling requires a Fresnel number $F_{1}$ corresponding to the holographic regime. Contrast transfer is high for all ranges of spatial frequencies which is ideal for phase retrieval. Again, it should be mentioned that even under ideal conditions (no vibration, perfect illumination, etc.) smaller Fresnel numbers limit the obtainable resolution to a value larger than a pixel.

\subsection{Direct phase retrieval: Dealing with lost information}

The previous sections have shown how the information content of a measurement depends on the detector position and how it is encoded in the intensity patterns. The following sections summarise several methods to retrieve this phase information from the holograms and complement them to reconstruct the desired object properties. To this end, first, the so-called single-step inversions are introduced, which yield a reconstruction obtained directly from the data through the application of a Fourier filter. If the objects meet a number of restrictive constraints, like negligible absorption or consistence of a single material, they can be retrieved to a certain degree from a single measurement alone. In contrast, iterative algorithms summarised in the following section alternatingly adapt a guess of the object under different constraints to find the best match for the data. With the drawback of larger computational effort, this allows to implement additional a priori constraints comparatively in a straight forward fashion and thus to obtain reconstructions from a larger class of objects. 


\subsubsection{Holographic phase retrieval}

In [57], GABOR describes a technique to measure a hologram and retrieve an approximation of the sample. First, he illuminated a weakly absorbing object, obtaining a wave field described as a small signal interfering with the virtually undisturbed illumination. At a distance $d$ behind the sample, he recorded the holographic intensity pattern with a photographic plate. The photography was developed to yield an absorption mask subsequently placed at the position of the photographic plate in the setup with the object removed. Now illuminated only by the reference wave, a reconstruction of the object at a distance $d$ behind the mask ( $2 \mathrm{~d}$ behind the original object position) can be recorded.

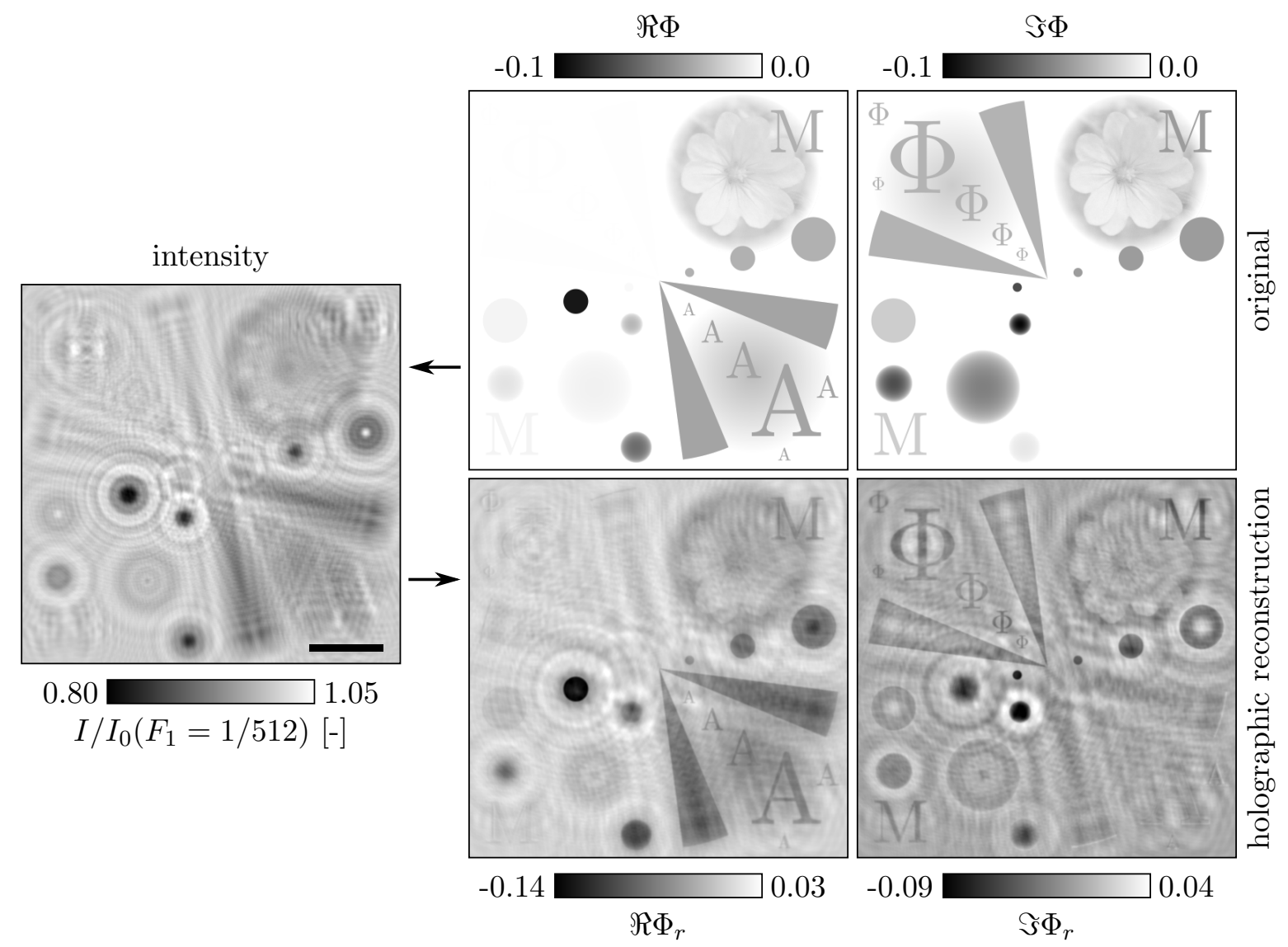

Figure 5.6: Example of a holographic reconstruction. To satisfy the requirement of a weak object, real and imaginary part of the linearised exit wave $\Psi \approx 1+\Phi$ vary less than $10 \%$. The backpropagation of the intensity in the object plane yields reconstructions where the appropriate details can be seen clearly, even the smallest letters can be recognised again. However, the propagated twin image (clearly visible in the originally empty regions) dramatically lowers the quality of the reconstruction. The scale bar indicates a width of 100 px, the Fresnel number has been chosen w.r.t. ideal numerical propagation.

In the following, the formation of this reconstruction will be described. The mask imprints intensity proportional to the original signal on the reference wave. If we 
consider the intensity as a superposition of image and twin image as described in Eq. $(5.13)$

$$
\frac{I}{I_{0}} \approx 1+e^{i k d} \mathcal{D}^{-d} \Phi^{*}+e^{-i k d} \mathcal{D}^{d} \Phi
$$

a second propagation about $d$ and multiplication with $\exp (-i k d)$ yields

$$
e^{-i k d} \mathcal{D}^{d}\left[\frac{I}{I_{0}}-1\right] \approx \Phi^{*}+e^{-2 i k d} \mathcal{D}^{2 d} \Phi
$$

Thus, this experimental reconstruction would focus the twin image, which is superposed by the object signal propagated about twice the original distance and spread out to form a defocused background signal in the ideal case. Equivalently, by a (numerical) propagation of the intensity measurement about $-d$, the original object can be focused, superposed by the defocused twin image. Such numerical reconstructions yield both amplitude and phase of the exit wave but they are usually disturbed by twin artefacts as illustrated in Fig. 5.6. Nevertheless, this holographic phase retrieval gives an idea of object size and composition, with the only restricting assumption of a weakly interacting object.

\subsubsection{CTF-based reconstruction}

For improved reconstructions with suppressed twin image artefacts, more information about the objects is required. Several phase-retrieval methods have been developed based on the free-space contrast transfer function (CTF) [8, 64-67]. If the object meets certain assumptions, it can be directly reconstructed based on the CTF formula derived in Eq. (5.18)

$$
\mathcal{F}\left[\frac{I}{I_{0}}-1\right] \approx-2 k(\bar{\delta} \sin (\varphi)+\bar{\beta} \cos (\varphi)) .
$$

One example of useful prior knowledge is a negligible absorption ("pure phase object"), yielding

$$
\bar{\beta}=0 \Rightarrow \bar{\delta} \approx \frac{-1}{2 k \sin (\varphi)} \mathcal{F}\left[\frac{I}{I_{0}}-1\right],
$$

or the assumption of a single material with $\kappa:=\beta / \delta$, resulting in

$$
\bar{\beta}=\kappa \bar{\delta} \quad \Rightarrow \quad \bar{\delta}=\frac{-1}{2 k(\sin (\varphi)+\kappa \cos (\varphi))} \mathcal{F}\left[\frac{I}{I_{0}}-1\right] .
$$

In both cases, the zeros of the sine and cosine functions prohibit the reconstruction - which is in fact a deconvolution - by simple division. Common workarounds are to spare small intervals around the zeros from the division [67] or to regularise the functions, e.g. by

$$
\frac{-1}{2 k \sin (\varphi)} \rightarrow \frac{-\sin (\varphi)}{2 k \sin ^{2}(\varphi)+k \varepsilon\left(\nu_{\perp}\right)}
$$


with the regularisation

$$
\varepsilon\left(\nu_{\perp}\right)=a_{1} f\left(\nu_{\perp}\right)+a_{2}\left(1-f\left(\nu_{\perp}\right)\right) \text { with } f\left(\nu_{\perp}\right)=\frac{1}{2}\left(1-\operatorname{erf}\left(\frac{\nu_{\perp}^{2}-\nu_{c u t}}{\sigma_{c u t}}\right)\right)
$$

as proposed in [68]. This function allows to regularise the low spatial frequencies suffering from poor contrast transfer differently than the high frequencies hampered by many zero crossings of the CTF. The free parameters $a_{1}, a_{2}, \nu_{\text {cut }}$ and $\sigma_{\text {cut }}$ have to be chosen empirically for each experiment. In practice, $\nu_{c u t}$ is set to the first maximum of the pCTF while $\sigma_{\text {cut }}=0.01$ periods per pixel yields good results. The reconstruction of a pure phase object shown in Fig. 5.7 clearly outperforms the holographic reconstruction but is still affected by artefacts from the missing spatial frequencies in the hologram. Without further knowledge about the sample, the missing frequencies have to be measured. A practical method is to modify the Fresnel number by changing the wavelength or the object-to-detector distance [69-71]. In practice, four different measurements for each projection are sufficient to reconstruct the object with the formula $[71-73]$

$$
\bar{\delta} \approx \frac{-\sum_{j} \mathcal{F}\left[I_{j} / I_{0}-1\right] \sin \left(\phi_{m}\right)}{2 k \sum_{m} \sin ^{2}\left(\phi_{m}\right)+k \varepsilon\left(\nu_{\perp}\right)} .
$$

However, additional measurements are not compatible with the goal of fast tomography unless acquired at the same time with several semi-transparent- or energydiscriminating detectors, further increasing the necessary peak radiation dose.

For Fresnel numbers in the direct contrast regime where the pCTF can be well approximated by a parabola, complementary reconstruction methods have been developed based on the transport of intensity equation (TIE). If the exit wave $\Psi_{0} \propto \sqrt{I_{0}} \exp \left(\Phi_{0}\right)$ can be expressed by an amplitude $\sqrt{I_{0}} \propto\left|\Psi_{0}\right|$ and phase shift $\Phi_{0}=\arg \left(\Psi_{0}\right)$, the detector image is given by [3]

$$
I(x, y, z=d) \propto I(x, y, z=0) \cdot\left(1-\frac{d}{k}\left[\partial_{x}^{2}+\partial_{y}^{2}\right] \Phi_{0}\right) .
$$

Reconstruction formulas are based on assumptions like a pure phase object (modified Bronnikov algorithm [74])

$$
\Phi_{0} \approx 2 \pi F_{1} \mathcal{F}^{-1}\left[\frac{\mathcal{F}\left(I / I_{0}-1\right)}{\nu_{\perp}^{2}+\varepsilon}\right],
$$

or a fixed $\kappa=\beta / \delta$ ratio of a single material [75] (set the regularisation parametre in Eq. (5.31) to $\left.\varepsilon=4 \pi F_{1} \kappa\right)$. Other methods reduce artefacts in the reconstructions if the former constraints are not strictly satisfied, notably the Bronnikov aided correction presented in [76]. In fact, the main challenge is the inversion of the second derivative of the TIE which can be carried out by a properly regularised multiplication with $\nu_{\perp}^{-2}$ in Fourier space acting as a low-pass filter. In this work, the function

$$
\tilde{u}\left(\nu_{\perp}\right)=\frac{1-\exp \left[-\varepsilon \cdot \nu_{\perp}^{2}\right]}{\nu_{\perp}^{2}} .
$$


has been used, lying in between the widely utilised filter function $1 / \nu_{\perp}^{2} \rightarrow 1 /\left(\nu_{\perp}^{2}+\varepsilon\right)$ from Eq. (5.31) and the Thikonov regularised CTF in Eq. (5.27).
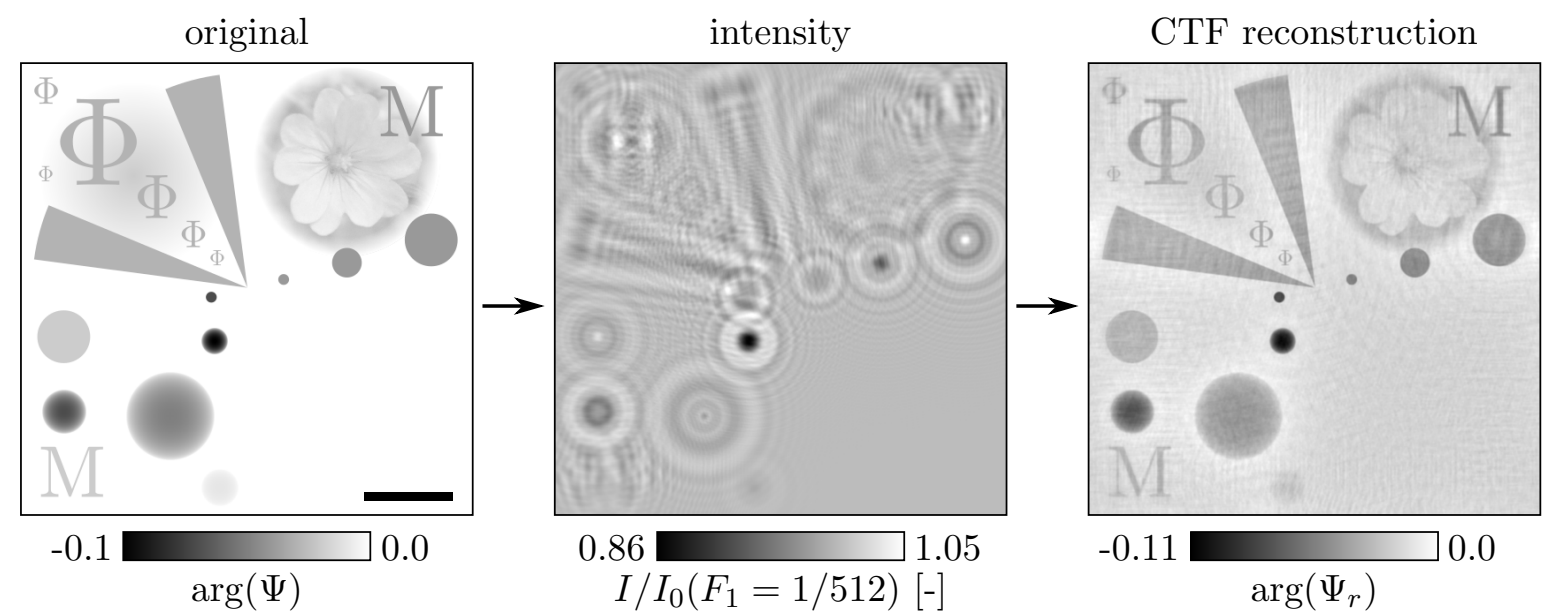

Figure 5.7: CTF reconstruction of a pure phase object with the regularisation parameters $a_{1}=0$ and $a_{2}=0.02$. All object features are clearly retrieved but still disturbed by a background originating from the missing spatial frequencies in the hologram. The scale bar indicates a width of $100 \mathrm{px}$.

\subsection{Iterative phase retrieval: Replacing lost information}

In chapter 3.4, the use of iterative procedures has been demonstrated in the context of tomographic reconstruction and sinogram retrieval. In general, these methods are well suited to find a solution to an inverse problem, i.e. searching a cause for the measured result. However, for arbitrary objects, the problem of phase retrieval is ill-posed, the retrieved object does not uniquely explain the data. In fact, an infinite number of objects can be thought to yield the same intensity measurement. Thus, further constraints are necessary. The main advantage of iterative procedures is that a large variety of such constraints can be applied to the reconstruction process, which in addition can be inspected and optimised during the execution. One example of additional knowledge not exploited so far is the negativity of the exit wave's argument. In other words, the phase in matter can only advance compared to vacuum and the amplitude can only decrease by energy absorption since we have excluded all interference effects within the object. The knowledge that $f(x, y) \leq 0$ requires the Fourier space representation $\mathcal{F}[f]$ to be negative semi-definite (BOCHNER's theorem) - a condition very difficult to enforce in Fourier space [77]. A second example is the knowledge of the compact support of the object $f$. We have seen in Chap. 3.4 that this corresponds to $\mathcal{F} f$ being invariant under certain convolutions. This condition is of great value: in [78] it has been shown that a compact support is sufficient for uniqueness in holographic phase retrieval. As we will see in this section, both constraints are easy to apply iteratively in real space with great benefit. 


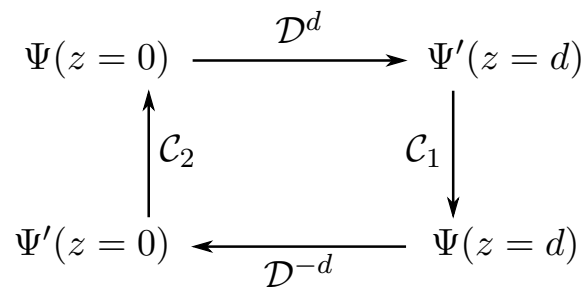

Figure 5.8: Basic scheme of an iterative algorithm for phase retrieval. A guess $\Psi$ of the wave field cycles between two positions on the optical axis in which the constraints $\mathcal{C}_{1}$ and $\mathcal{C}_{2}$, respectively, have to be satisfied. See the text for details.

The application of iterative techniques in X-ray optics is based on an approach by Gerchberg and Saxton, who described a solution to the phase problem in [79]. The original work is based on the premise that the intensity $|\Psi|^{2}$ is not only known in the detection plane but also in a second, sufficiently distant plane. This can be achieved either by a second measurement but also by a priori assumptions like those of a pure phase object. In the latter case, the intensity in the object plane is known to equal the illumination, which can be measured or approximated by a constant in case of a clean, parallel beam illumination. Thus, $|\Psi(z=0)|^{2}$ and $|\Psi(z=d)|^{2}$ are known. In [79], the detection plane is so far removed from the object plane that they are connected by the Fourier transform, i.e. $\Psi(z=d)=\mathcal{F} \Psi(z=0)$. This special case is called PAULI-problem after its first formulation in quantum mechanics. However, the technique can also be applied to near-field experiments with $\Psi(z=d)=\mathcal{D}^{d} \Psi(z=0)$ if the intensity patterns differ sufficiently. The iterative scheme is sketched in Fig. 5.8 and can be described in operator notation by

$$
\Psi_{m+1}(z=0)=\left(\mathcal{C}_{2} \mathcal{D}^{-d} \mathcal{C}_{1} \mathcal{D}^{d}\right) \Psi_{m}(z=0)
$$

An initial guess $\Psi_{0}$ is propagated to the detection plane by $\mathcal{D}^{d}$. There, a so-called modulus-constraint

$$
\mathcal{C}_{1}: \Psi_{m}(z=d)=\sqrt{I(z=d)} \Psi_{m}^{\prime}(z=d) /\left|\Psi_{m}^{\prime}(z=d)\right|
$$

is applied, replacing the amplitude by the measured values $\sqrt{I(z=d)}$ while keeping the phase information. After the backpropagation $\mathcal{D}^{-d}$, a second modulus constraint $\mathcal{C}_{2}$ restoring the measured or estimated intensity is applied in the object plane. These steps are repeated iteratively, causing a monotonous convergence of $\Psi_{m}$ to the true value with the number of iterations $m$. This scheme can be easily extended to include additional detector distances. In addition to the intensity in one or more planes, an arbitrary number of further constraints can be incorporated. For the negativity condition in the object plane mentioned above

$$
\begin{aligned}
& \beta \geq 0 \Rightarrow \quad\left|\Psi_{m}(z=0)\right| \leq\left|\Psi_{i}\right| \\
& \delta \geq 0 \Rightarrow \quad \arg \left(\Psi_{m}(z=0)\right) \leq \arg \left(\Psi_{i}\right),
\end{aligned}
$$


the amplitude cannot be larger than those of the illumination $\Psi_{i}$ and the phase shift has to be negative. For a unit amplitude plane wave illumination $\left|\Psi_{i}\right|=1$ and $\arg \left(\Psi_{i}\right)=0$ with the abbreviation $\Psi_{m}^{\prime}(z=0)=\mathcal{D}^{-d} \mathcal{C}_{1} \mathcal{D}^{d} \Psi_{m}(z=0)$ this can be ensured e.g. by

$$
\Psi_{m+1}(z=0)=\min \left(1,\left|\Psi_{m}^{\prime}(z=0)\right|\right) \cdot \exp \left(i \min \left[0, \arg \left(\Psi_{m}^{\prime}(z=0)\right)\right]\right) .
$$

Even though this constraint can be applied to virtually all samples discussed here, it is not sufficient for a proper reconstruction. One or several additional assumptions like negligible absorption, slowly varying phase shifts, known relationships between phase shift and absorption, or known compact support of the object are required to compensate the lost information in a hologram. In case of a finite support $\Omega$, the illumination in the object plane outside $\Omega$ is unperturbed, leading to the iteration step

$$
\Psi_{m+1}(z=0)=\left\{\begin{array}{cl}
\Psi_{m}^{\prime} & \text { if }(x, y) \in \Omega \\
1 & \text { else. }
\end{array}\right.
$$

Alternatively, the sample can be prepared in such a way that the properties of several regions are known. For example, by mounting it in a frame absorbing the X-rays everywhere outside $\Omega$, the wave field can be found by requiring

$$
\Psi_{m+1}(z=0)=\left\{\begin{array}{cl}
\Psi_{m}^{\prime} & \text { if }(x, y) \in \Omega \\
0 & \text { else }
\end{array}\right.
$$

This algorithm is known as Error-Reduction (ER) procedure introduced by FIENUP [80]. Since this algorithm is known to stagnate in a local minimum of the solution space, often a Hybrid-Input-Output (HIO) scheme is utilised, which may overcome this problem $[3,80]$ with

$$
\Psi_{m+1}(z=0)=\left\{\begin{array}{cl}
\Psi_{m}^{\prime}, & \text { if }(x, y) \in \Omega \\
\Psi_{m}-\beta^{\prime} \Psi_{m}^{\prime} & \text { else. }
\end{array}\right.
$$

Here, $\beta^{\prime}$ is a parameter between 0.5 and 1 . A first guess of the phase $\Psi_{0}$ is often provided by random numbers. It can be beneficial to repeat the entire process with different guesses and average the final results, as unlucky choices of initial phase can still lead to stagnation in local minima [3]. In [81] a similar algorithm with improved performance, relaxed averaged alternating reflections (RAAR), is presented and analysed mathematically to obtain a strategy for adjusting $\beta_{m}^{\prime}$ during the reconstruction. An example of iterative phase retrieval is shown in Fig. 5.9 after 1000 iterations. Left, the same data as in the CTF example (see Fig. 5.7) has been reconstructed with the assumptions of a pure phase object and a negative phase shift. On the right, the same data as shown in 5.2 has been reconstructed, clearly exceeding the assumption of a weak object. With a given rough support identical for phase and amplitude (not shown) and the negativity constraint, this class of objects can be reconstructed, illustrating the capabilities of the iterative scheme. 
phase object, 1000 it.

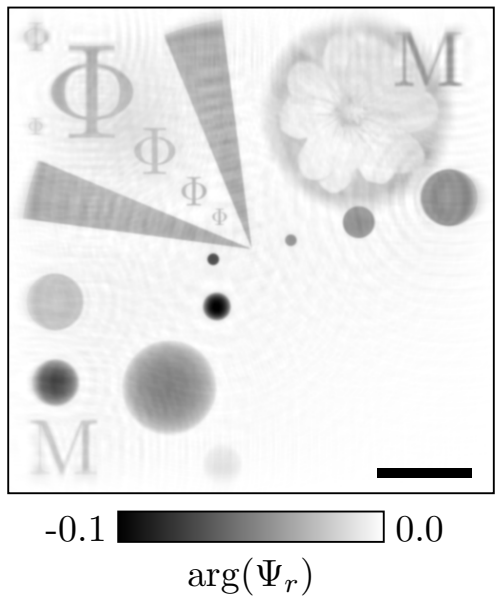

mixed object, 1000 iterations

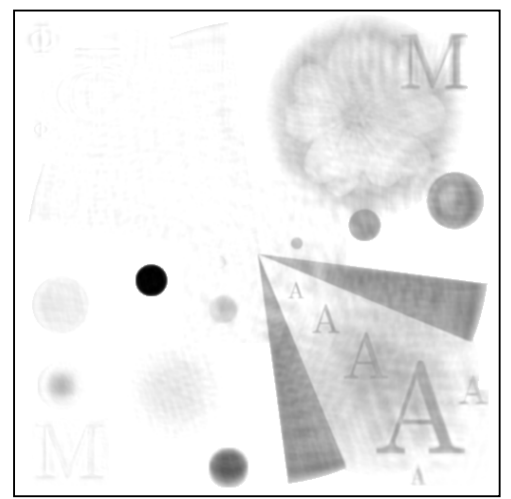

0.0

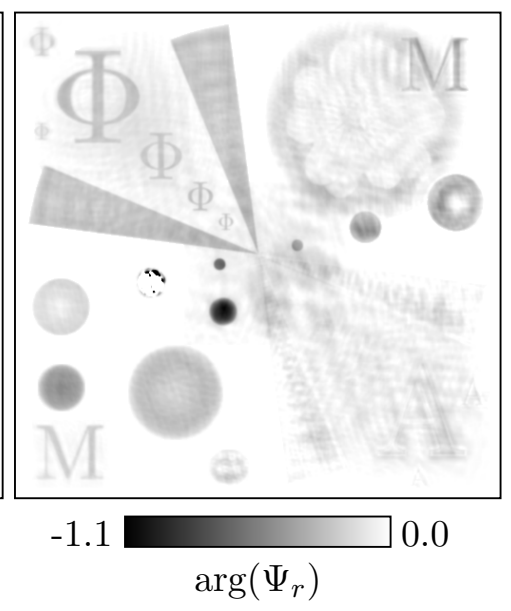

Figure 5.9: Result of an iterative reconstruction after 1000 iterations. The pure phase object (left) as well as a strong object (right) could be reconstructed to a degree that fine details are clearly visible. See text for details about the constraints. The scale bar indicates a width of $100 \mathrm{px}$, the Fresnel number $F_{1}=1 / 512$ has been chosen in accordance with ideal numerical sampling.

\subsection{Phase retrieval in $3 d$}

In this chapter, we bring together tomography and phase retrieval, i.e. the $3 \mathrm{~d}$ structure of a sample is to be reconstructed from a set of holograms recorded for different projection angles. The prototypical setup is sketched in Fig. 5.10. A plane wave $\Psi_{0}$ illuminates a weakly interacting object resulting in an exit wave in the $x-y$-plane as described by the projection approximation. The free-space propagation distance to the detector is large enough for the formation of holographic intensity patterns with $F_{a}<1$. The object is rotated around the $y$-axis and holograms are measured at equidistant angular steps over a range of at least $180^{\circ}$. In operator notation, the intensity $I_{\alpha_{m}}$ for the angle $\alpha_{m}$ can be described by

$$
I_{\alpha_{m}}=\left|\mathcal{D}^{d} \Psi_{0} \exp \left(i k \mathcal{P}_{\alpha}(1-n)\right)\right|^{2}
$$

The reconstruction usually inverts the order of the experimental steps. First, the complex exit waves are retrieved for all projections individually by an appropriate phaseretrieval method, followed by the tomographic reconstruction mostly implemented by a FBP $[74,82-86]$. However, the application of Fourier filters for direct (TIE/CTF) phase retrieval and filtered backprojection are occasionally combined to a single step for increased performance $[82,87]$. As demonstrated above, the main challenge of the reconstruction is the phase retrieval usually depending on restrictive constraints or on a priori knowledge not necessarily available. Thus we asked if a combination of both inverse problems, phase retrieval and tomographic reconstruction, can help to lower these requirements, meaning if - and to which extent - tomographic consistency as introduced in Chap. 3.4.1 can be used as a constraint for phase retrieval. 


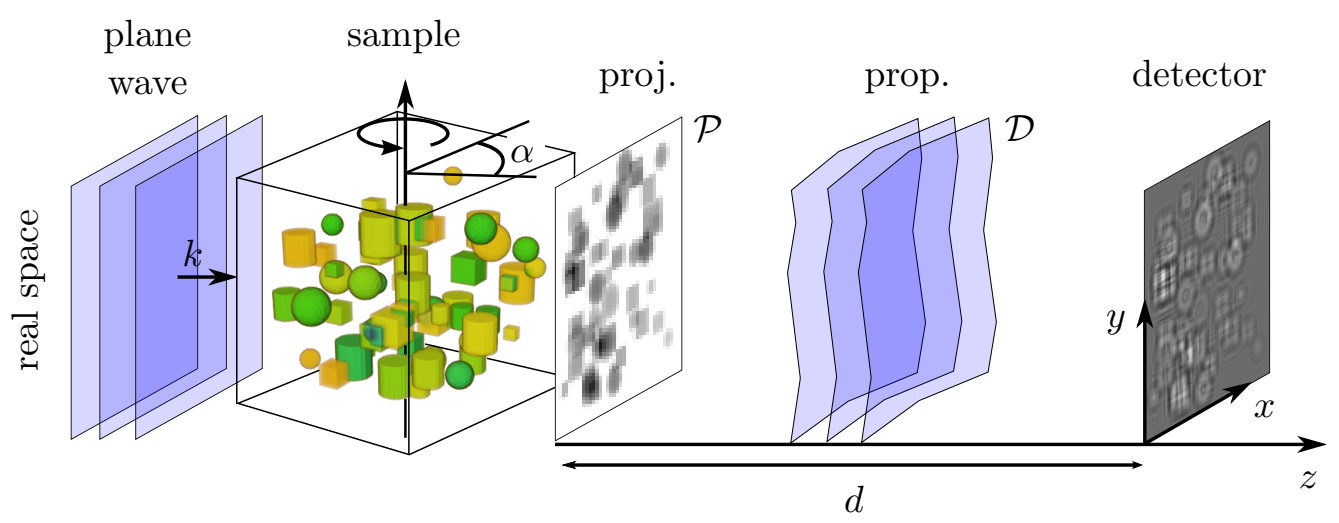

Figure 5.10: Typical setup for phase-contrast tomography. A plane wave illuminates the object, the exit wave given by the projection approximation propagates to the detector in free space yielding a hologram for each projection angle $\alpha_{m}$.

In the first subsection, a procedure named iterative reprojection phase-retrieval (IRP) is presented, combining iterative phase retrieval with the algebraic reconstruction technique for tomography. It is based on a strictly three-dimensional representation of the object, leading to significantly improved reconstructions with considerably less input of a priori knowledge on the sample, even in the presence of noise. To complement the publication of this scheme in [10], the method is introduced here from the perspective of ART, not iterative phase retrieval like in the original presentation. The gain in reconstruction quality is illustrated as a function of the number of projections as well as for experimental data. In the subsequent section, closely based on the related publication [49], a fast and practical iterative method for the special case of a weakly interacting object is presented, based on the three-dimensional propagation introduced in chapter 4.4. Finally, both methods are compared with the result of the common sequential approach on an experimental dataset of freeze-dried Deinococcus radiodurans bacteria.

\subsubsection{Consistency as a constraint: Iterative reprojection phase-retrieval}

The basic idea of IRP is sketched in Fig. 5.11 as a modified ART algorithm. As such, it cycles between the $3 \mathrm{~d}$ object representation and the projections, both connected via projection- $\mathcal{P}$ and backprojection operator $\mathcal{P}^{-1}$, as well as between object- and detection plane, expressed by propagation $\mathcal{D}$ and backpropagation $\mathcal{D}^{-1}$.

Starting in the object plane, a $3 \mathrm{~d}$ guess of the sample $n$ is projected numerically to the same $N_{\alpha}$ angles $\alpha_{j}$ as measured in the experiment. Then, all projections are propagated to the detection plane, where the modulus constraint $\mathcal{C}_{1}$ is applied. Matching the measured amplitudes, all projections are back-propagated to the object plane and a positivity condition $\mathcal{C}_{2}$ for $\beta$ and $\delta$ ensures conformity with the projection approximation. Since the ART in the given implementation only works for strictly positive (or strictly negative) values, only in the next step the results are compared with the direct projections to obtain corrected values. In the final step, these corrections are 


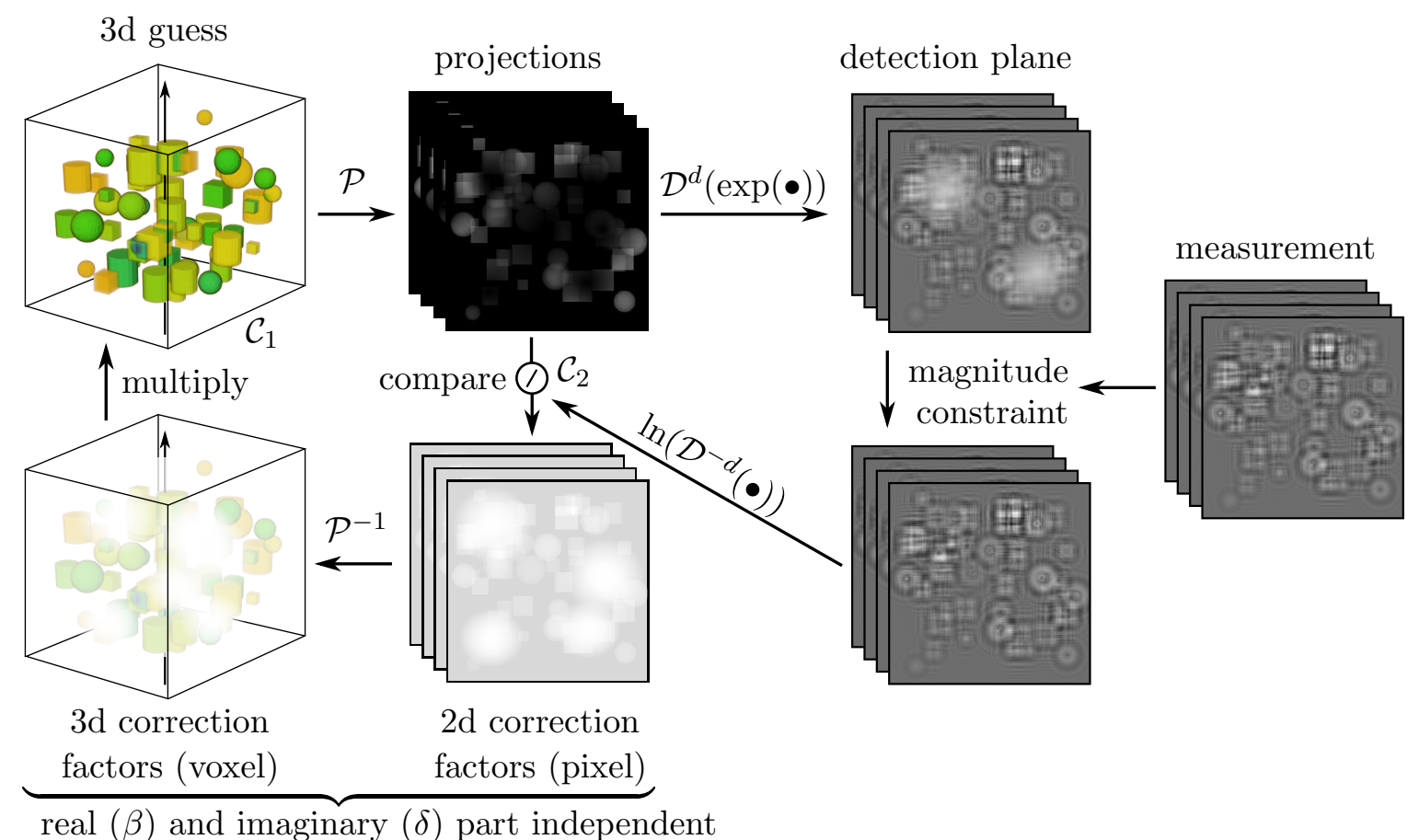

Figure 5.11: Principle of iterative reprojection phase-retrieval (IRP) expressed as a modified ART algorithm. See text for details.

back-projected forming a correction volume which is applied to the guess in $3 \mathrm{~d}$. In operator notation, a single iteration is thus given by

$$
\begin{aligned}
\delta(x, y, z)_{m+1} & \equiv \Re\left(1-n_{m+1}\right) \\
& =\Re\left(1-n_{m}\right) \cdot \frac{1}{N} \sum_{j=1}^{N} \mathcal{P}_{\alpha_{j}}^{-1} \frac{\Re\left\{\mathcal{C}_{2} \ln \left[\mathcal{D}^{-d} \mathcal{C}_{1} \mathcal{D}^{d} \exp \left(-i k \mathcal{P}_{\alpha_{j}}\left(1-n_{m}\right)\right)\right]\right\}}{\Re\left\{-i k \mathcal{P}_{\alpha_{j}}\left(1-n_{m}\right)\right\}},
\end{aligned}
$$

and analogously for the imaginary part $\beta(x, y, z)_{m+1} \equiv \Im n_{m+1}$. This describes a slowly converging implementation of ART like SIRT, with preference given to quality over reconstruction speed. Compared to the FBP it has the advantage of introducing fewer high frequency artefacts, which would otherwise spoil the overall reconstruction [88]. A particular benefit of averaging all projections before updating the guess is a higher robustness against single flawed projections which could otherwise alter the volume to a non-converging state. This can happen e.g. by multiplying voxels by zero such that their value can never be changed again in the multiplicative scheme. To prevent this, a positivity condition $\mathcal{C}_{2}$ is implemented as

$$
\mathcal{C}_{2}: \Psi_{m}(z=0)=\left|\Re \Psi_{m}^{\prime}(z=0)\right|+i\left|\Im \Psi_{m}^{\prime}(z=0)\right|
$$

with the abbreviation $\Psi_{m}^{\prime}(z=0)=\ln \left[\mathcal{D}^{-d} \mathcal{C}_{1} \mathcal{D}^{d} \exp \left(-i k \mathcal{P}_{\alpha}\left(1-n_{m}\right)\right)\right]$. Through this update step, it is guaranteed that $\Psi_{m}$ is closer to the real value (which is $\leq 0$ ) than 
before without setting values to zero. While convergence and properties of similar iterative algorithms have been well studied and have been continuously adapted and generalised with respect to the employed constraints, one decisive difference compared to earlier work on phase retrieval has to be stressed. The object is strictly represented as a 3d object, intrinsically guaranteeing tomographic consistency of the projection. This iterative $3 \mathrm{~d}$ reconstruction scheme which obeys the consistency constraint is denoted as iterative reprojection phase-retrieval (IRP).

Clearly, the scheme can be modified in many ways to adapt to the problem as well as possible. For example, the left multiplicative 'tomography circle' in Fig. 5.11 can be replaced by the additive SIRT scheme or improved methods for faster convergence. Furthermore additional constraints can be applied at any step. Importantly, a new class of $3 \mathrm{~d}$ constraints lends itself to be enforced on the $3 \mathrm{~d}$ representation of the object. Features such as known holes in the sample or shells composed of different materials in each layer cannot be modelled in projections but can easily be enforced in the $3 \mathrm{~d}$ volume. Moreover, the execution order of the algorithm parts may be optimised for faster convergence or better suitability to certain problems. For example, the tomography loop can be carried out several times to improve consistency before the next modulus update. The right 'phase-retrieval'-loop with the positivity constraint $\mathcal{C}_{2}$ can also be iterated several times between consecutive tomography steps to improve agreement with the measured data. As shown in [10], the number of such inner iterations can be optimised in terms of an error metric, e.g.

$$
\sigma^{2}:=\frac{1}{N_{\alpha}} \sum_{\alpha_{j}, x, y}\left(\left|\Psi_{n, \alpha_{j}}^{\prime}(z=d)\right|-\sqrt{I_{\alpha_{j}}}\right)^{2},
$$

comparing the modulus of the propagated projections $\Psi^{\prime}(z=d)$ with the recorded detector images $I_{\alpha_{j}}$. Monitoring the error is also useful to stop the algorithm either by falling below a threshold or at stagnating convergence.

As demonstrated in [10], IRP clearly yields superior reconstruction quality compared to the sequential approach and converges much faster with respect to the number of phase-retrieval loops. Low spatial frequencies in particular, which are poorly transferred and only loosely constrained otherwise, can be retrieved better. According to the Fourier slice theorem, they are most coupled in tomography. Averaging over all projections makes the scheme robust against noise and the possibility of including new 3d constraints can help to sufficiently reconstruct objects from datasets previously impossible to retrieve due to noise, sparsity, or missing projection angles. However, in the following example, none such optimisations have been used, only the scheme as sketched and explained above, to demonstrate the benefit gained by requiring consistency alone. For this, a sample consisting of $256^{3}$ voxels has been designed containing spheres and cubes with randomly varying diameter and $\beta / \delta$ ratios from 0 to $\beta / \delta=0.15$. The object has been projected to $N_{\alpha}$ angles equidistantly distributed over a range of $180^{\circ}$ and propagated to a detection distance with $F_{1}=0.01$. Fig. 5.12 shows the IRP result for $\alpha=0^{\circ}$ after 1000 iterations for different numbers of angles $N_{\alpha}$. For $N_{\alpha}=1$ tomography is an empty operation - for an angle of $0^{\circ}$ not even an interpolation takes place. This demonstrates the quality of conventional phase retrieval where all projections are treated separately. For $N_{\alpha}=2$ angles, the background increases without any 


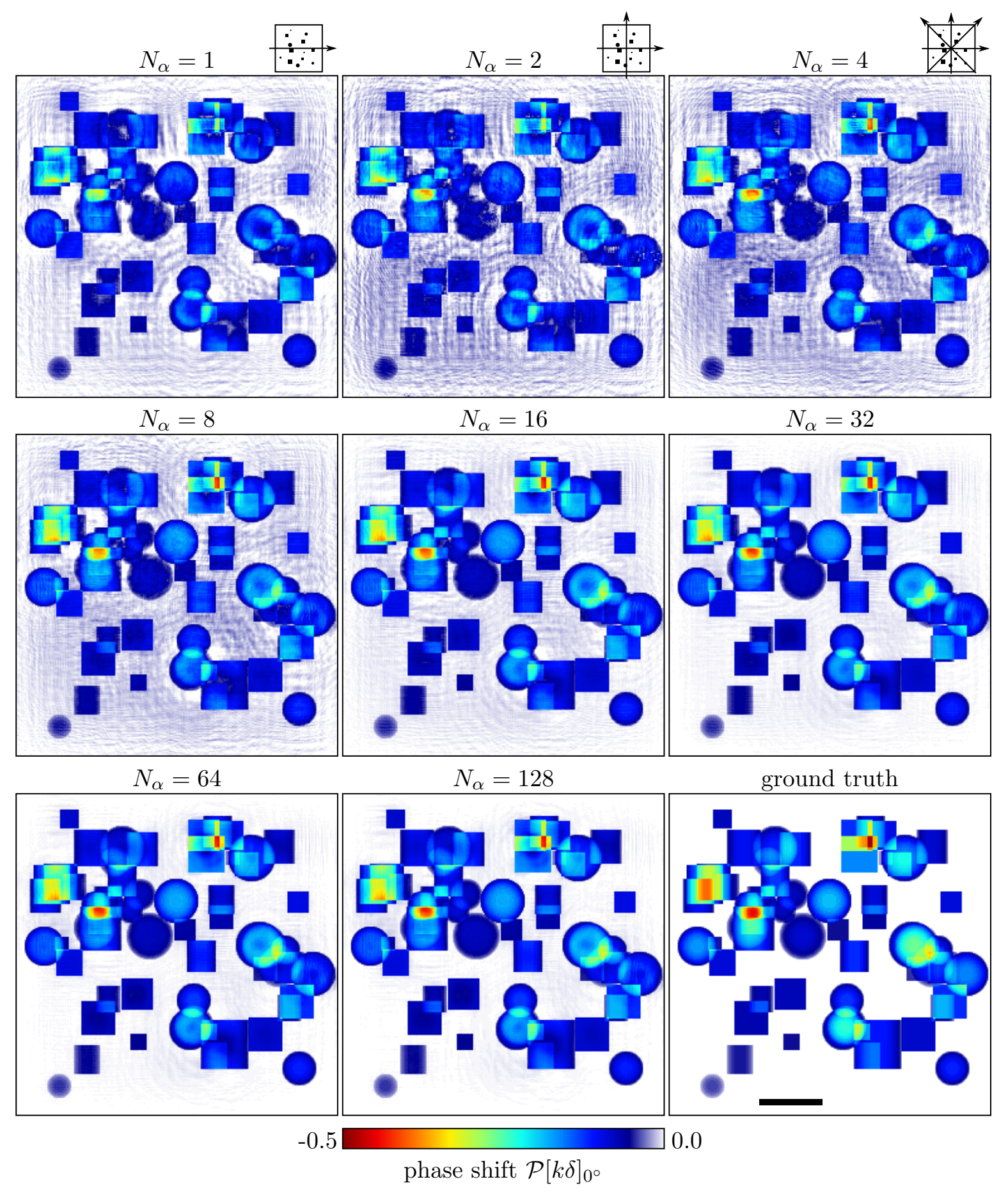

Figure 5.12: IRP results after 1000 iterations for different numbers of projections $N_{\alpha}$. Clearly, an increase in reproduction quality of the ground truth in the last image can be recognised. This can be attributed only to the consistency constraint. See the text for details.

improvement in the signal since the projections are orthogonal to each other and their coupling is minimal. However, the difference to the previous case shows a dependency 
even for this situation. The growing number of projections $N_{\alpha}$ with increasing coupling of the projections then clearly enables improved reconstructions with only minor differences to the ground truth, mainly in regions with large intensity. This improvement can only be attributed to the enforced consistency during phase retrieval, showing the value of this constraint. The final FBP of a conventional scheme decreases the noise but is not capable to achieve a comparable quality from projections as depicted for $N_{\alpha}=1$ [10]. Fig. 5.13 depicts reconstructions with the same parameters as before but with Gaussian noise added to the simulated intensities corresponding to a mean of 6000 photons per pixel. A similar improvement as before is observed. Even the high intensity regions are well reconstructed.

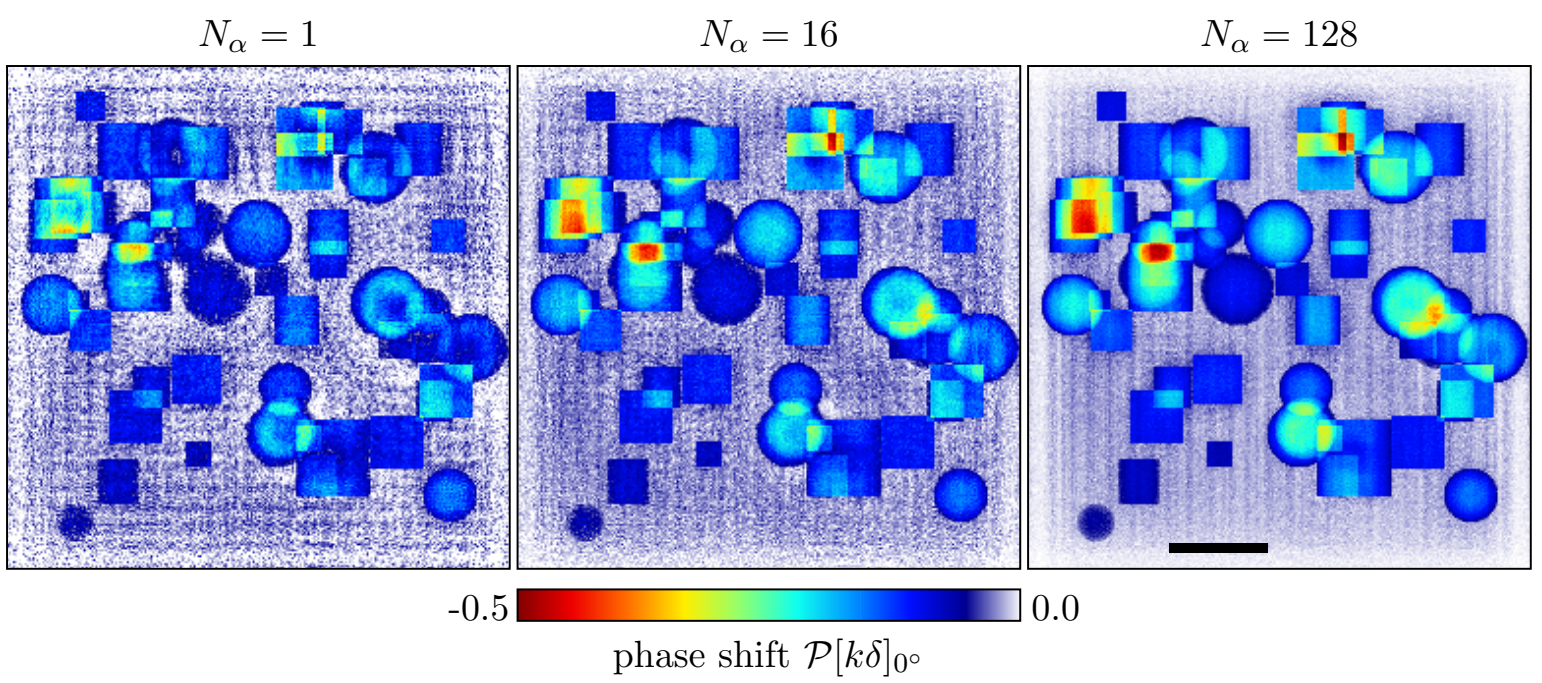

Figure 5.13: IRP results after 1000 iterations for different numbers of projections $N_{\alpha}$ with added noise. Again, an increase in reproduction quality can be recognised, which is attributed to the consistency condition. See the text for details.

\subsubsection{3d propagation-based phase retrieval}

The gain in quality achieved by IRP and similar schemes [11] goes along with the drawback of high numerical costs since extensive tomography and re-projection steps have to be carried out in each iteration. In this section, a method for weak objects is presented which considerably accelerates the combined reconstruction without sacrificing result quality. It is based on the $3 \mathrm{~d}$ propagation introduced in chapter 4.4. The object has to fulfil the same conditions as for the CTF which, in good approximation, are valid for a large variety of samples. Like IRP, the method is not restricted to small propagation distances but treats the problem for general Fresnel numbers including the holographic regime.

As introduced in Eq. 5.13, in the required weak object approximation the detector intensity images $I /\left|\Psi_{0}\right|^{2} \approx 1+\Phi_{d}+\Phi_{d}^{*}$ can be interpreted as the propagated image $\Phi_{d}$ superposed by the twin image $\Phi_{d}^{*}$. In chapter 4.4 it was demonstrated that the propagated image can be obtained by a projection from a $3 \mathrm{~d}$ propagated object in this 


\section{Propagation-based phase-contrast imaging}

limit. Thus, the intensity measurements can be considered as projections from a propagated $3 \mathrm{~d}$ object which is superposed by an inversely propagated, complex conjugated twin-object

$$
\frac{I_{\alpha}(z=d)}{\left|\Psi_{0}\right|^{2}}-1=\mathcal{P}_{\alpha}\left[e^{i k d} \mathcal{D}_{3 d}^{-d} \Phi_{3 d}^{*}+e^{-i k d} \mathcal{D}_{3 d}^{d} \Phi_{3 d}\right]=: \mathcal{P}_{\alpha}\left[I_{3 d}(z=d)\right]
$$

This formulation of the twin-image problem in $3 \mathrm{~d}$ shows that the intensity images $I_{\alpha}$ are consistent with respect to tomography and that the $3 \mathrm{~d}$ intensity volume $I_{3 d}$ can be obtained directly from the normalised measurements $I_{\alpha} / I_{0}-1$ by an inverse Radon transform. A 3d backpropagation then yields a sharpened object superposed by a defocused twin, analogous to the holographic reconstruction. In terms of the CTF in Fourier space, the $3 \mathrm{~d}$ intensity volume is given by

$$
\mathcal{F}\left[I_{3 d}(z=d)\right] \approx-2 k \mathcal{F}_{3 d}[\delta] \sin (\varphi)-2 k \mathcal{F}_{3 d}[\beta] \cos (\varphi)
$$

with $\varphi=d\left(\nu_{x}^{2}+\nu_{y}^{2}+\nu_{z}^{2}\right) /(2 k)$ generalised to three dimensions. In analogy to the $2 \mathrm{~d}$ case, the division by a properly regularised $3 \mathrm{~d}$ pCTF can be used to retrieve the object in the case of a pure phase object

$$
\delta(x, y, z) \approx \mathcal{F}^{-1}\left[-\frac{\sin (\varphi)}{2 k \sin ^{2}(\varphi)+k \varepsilon} \mathcal{F}\left[I_{3 d}(z=d)\right]\right]
$$

or in case of a fixed $\kappa=\beta / \delta$ ratio

$$
\delta(x, y, z) \approx \mathcal{F}^{-1}\left[-\frac{\sin (\varphi)+\kappa \cos (\varphi)}{2 k(\sin (\varphi)+\kappa \cos (\varphi))^{2}+k \varepsilon} \mathcal{F}\left[I_{3 d}(z=d)\right]\right] .
$$

The equations show that the phase problem does not introduce tomographic inconsistencies. Hence, the requirement for consistency cannot be used to improve phase retrieval directly. In particular, only limited improvements for phase retrieval can be expected by deterministic one-step algorithms like a direct CTF inversion if carried out in $3 \mathrm{~d}$ rather than $2 \mathrm{~d}$, even if the geometry of the $3 \mathrm{~d}$ problem is different from $2 \mathrm{~d}$ imaging $[55,89]$. The $3 \mathrm{~d}$ CTF with zero values on spherical shells does not contain all information about the object. Therefore, further constraints have to be applied, i.e. a priori information such as positivity, range restrictions of the object functions, or known support of the object - constraints that have been shown to be applied easily in iterative phase-retrieval procedures. In the previous section it has been shown that the performance of iterative phase retrieval with constraints such as positivity can be considerably improved if tomographic consistency is enforced, preventing projections from losing their initial consistency during individual phase retrieval. Thus, we expect similar improvements for iterative algorithms like ER, HIO or RAAR if they are carried out in $3 \mathrm{~d}$ with the concept of propagating volumes rather than individual phase retrieval for each projection, followed by a tomographic reconstruction. If the phase retrieval is carried out in $3 \mathrm{~d}$ according to the concept presented, consistency is guaranteed automatically.

The results of a simulation created to validate and to illustrate $3 \mathrm{~d}$ iterative phase retrieval, are shown in Fig. 5.14. A sample was designed consisting of 30 spheres with 
a diameter of $30 \mathrm{px}$ each, distributed randomly on a $256^{3}$ voxel grid with a fixed ratio $\beta / \delta=0.04$. The maximum phase shift of all projections was chosen to be $1.89 \mathrm{rad}$, which clearly exceeds the assumption of a weakly interacting object but demonstrates that the advantage of $3 \mathrm{~d}$ reconstruction persists even to larger phase shifts. To simulate the measured data in the detection plane, the intensities of projections propagated to the near field at a Fresnel number $F_{1}=0.05$ were computed for 402 equidistant angles in the range $0^{\circ} \leq \alpha \leq 180^{\circ}$ to satisfy the angular sampling criterion. This choice corresponds to a relevant experimental setting in X-ray phase contrast tomography with a photon energy of $12.4 \mathrm{keV}$, an effective pixel size of $10 \mathrm{~nm}$ and an effective sample-to-detector distance of $20 \mu \mathrm{m}$. The iterative phase retrieval cycled between object and detection position. In the detection plane, the common modulus constraint was applied while in the object plane, positivity was imposed by

$$
\Phi^{\prime}=1-\left|\operatorname{Re}\left(\Phi_{g}^{\prime}\right)-1\right|-i\left|\operatorname{Im}\left(\Phi_{g}^{\prime}\right)\right|
$$

This step was succeeded by a soft coupling $0 \leq \beta \leq 0.1 \delta$ of phase shift and absorption, implemented as follows: First, $\delta$ was set to the values of $\beta$ if $\beta>\delta$. Then, $\beta$ was limited to $\beta=0.1 \delta$ wherever larger values were observed. This again demonstrates the flexibility of iterative schemes and applies to a wide range of soft matter samples in the mentioned region of photon energy. 1000 iterations of this scheme were carried out for the conventional approach with each detector image reconstructed individually followed by a filtered backprojection (2d reconstruction) and for the $3 \mathrm{~d}$ volume starting with a filtered backprojection of the detector images. The results depicted in Fig. 5.14 clearly show the gain in quality of the $3 \mathrm{~d}$ approach due to enforced consistency. 


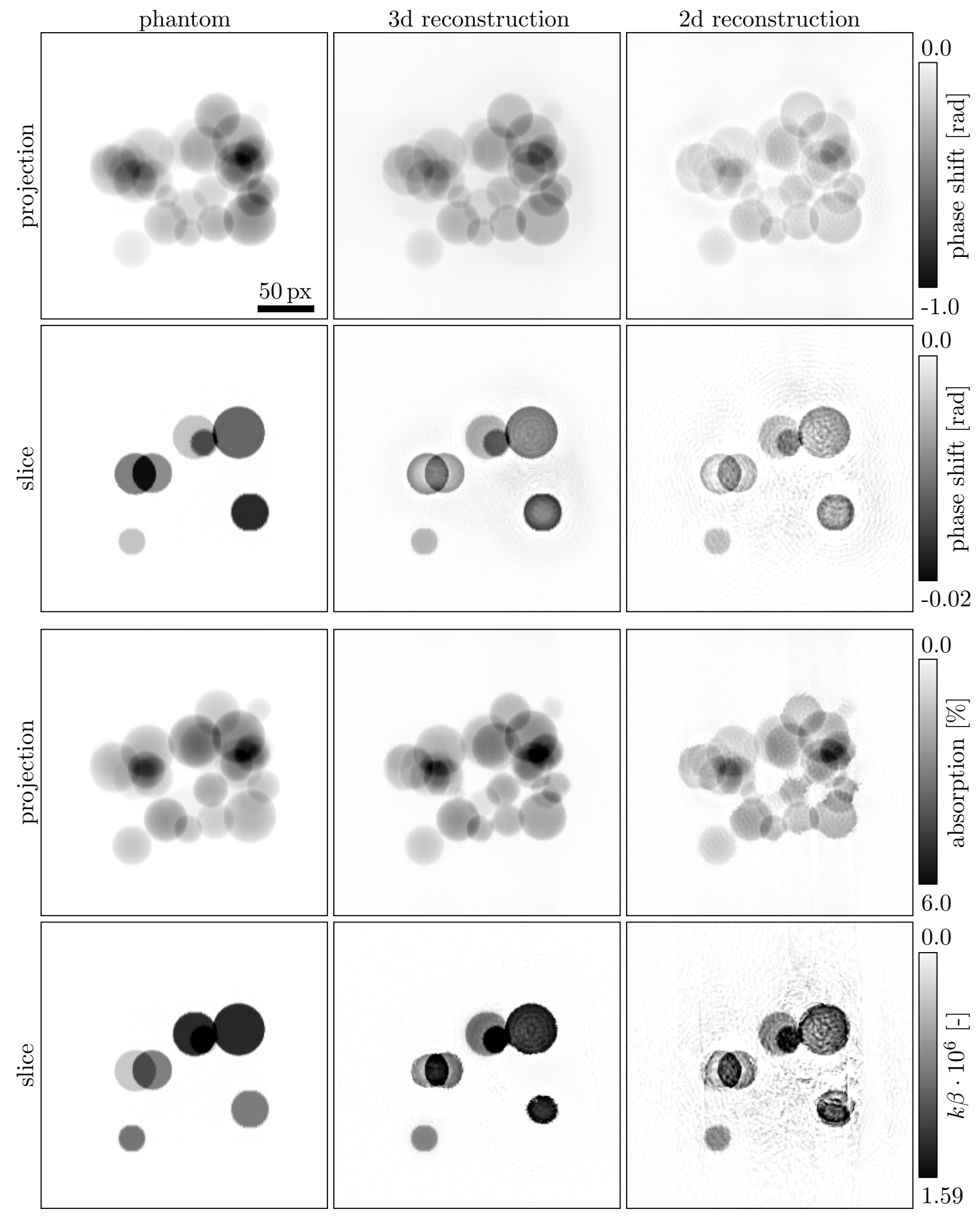

Figure 5.14: Comparison of phase shift and absorption results of the conventional (2d propagation) iterative reconstruction with those of the $3 \mathrm{~d}$ propagation approach. Depicted are typical projections of the phantom and the reconstructed volumes, as well as $x-z$ slices normal to the axis of rotation. The $3 \mathrm{~d}$ approach obtains a better reconstruction of the data with the same positivity constraint as the conventional scheme. The scale bar applies to all images. The figure is adapted from [49]. 


\subsubsection{Performance on experimental data}

The value of consistency as a constraint in phase retrieval has been exploited for the reconstruction of experimental data in $[73,90]$. IRP has been shown to outperform the results obtained by the conventional procedure, see [73] for details. Both reconstructions can be compared in Fig. 5.15.
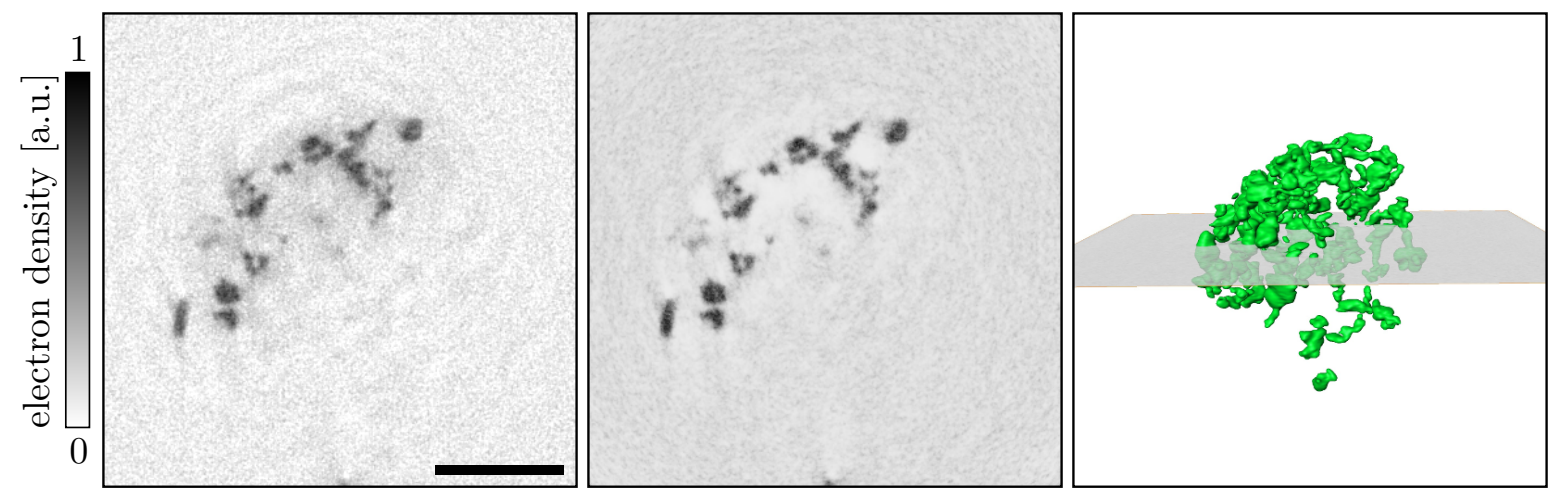

Figure 5.15: Slice through a $\mathrm{BaSO}_{4}$-labelled macrophage embedded in agarose reconstructed with conventional CTF-based phase retrieval followed by a filtered backprojection (left) and IRP (centre). A 3d rendering of the IRP result is depicted in the right image. The scale bar denotes $5 \mu \mathrm{m}$. Data adapted from [73].

To compare the quality of IRP with the $3 \mathrm{~d}$ propagation method on experimental data, both algorithms have been tested on a small holographic dataset of bacteria published previously in [91]. The data has been recorded by tomography in cone beam illumination with geometric magnification and phase contrast formation by free space propagation. The sample consisted of freeze-dried Deinococcus radiodurans bacteria, dispersed on an ultra-thin $\mathrm{Si}_{3} \mathrm{~N}_{4}$ membrane. It was illuminated by $13.8 \mathrm{keV}$ radiation exiting from an X-ray waveguide acting as a quasi-point source $8 \mathrm{~mm}$ in front of the sample, resulting in an effective pixel size of $83 \mathrm{~nm}$. The tomographic scan comprised of 83 projection angles distributed over $162^{\circ}$. See [91] for further experimental details. A typical holographic intensity image is shown in Fig. 5.16.

In the original publication [91] the data had been treated by a sequential scheme, first using phase retrieval based on a modified Hybrid-Input-Output algorithm (mHIO) $[91,92]$ with a support constraint, followed by FBP of all reconstructed projections. A major improvement over previous techniques was the automatic support determination for all projection angles. A slice through the reconstruction volume is shown in Fig. 5.16 for the original sequential reconstruction (mHIO). Note that despite the support constraint, the mHIO result shows artefacts outside the original support, indicating tomographic inconsistency of the projections. For IRP, 1000 iterations of the scheme described above have been carried out, with positivity and consistency as the only constraints. The result displays a much more homogeneous density distribution compared to mHIO, where the signal is concentrated at the top and bottom regions of the sample near the axis of rotation. Since there is no reason to assume that these regions in particular have a higher density than other parts of the sample, we attribute this enhancement of signal to inconsistency artefacts of the conventional mHIO method. The 


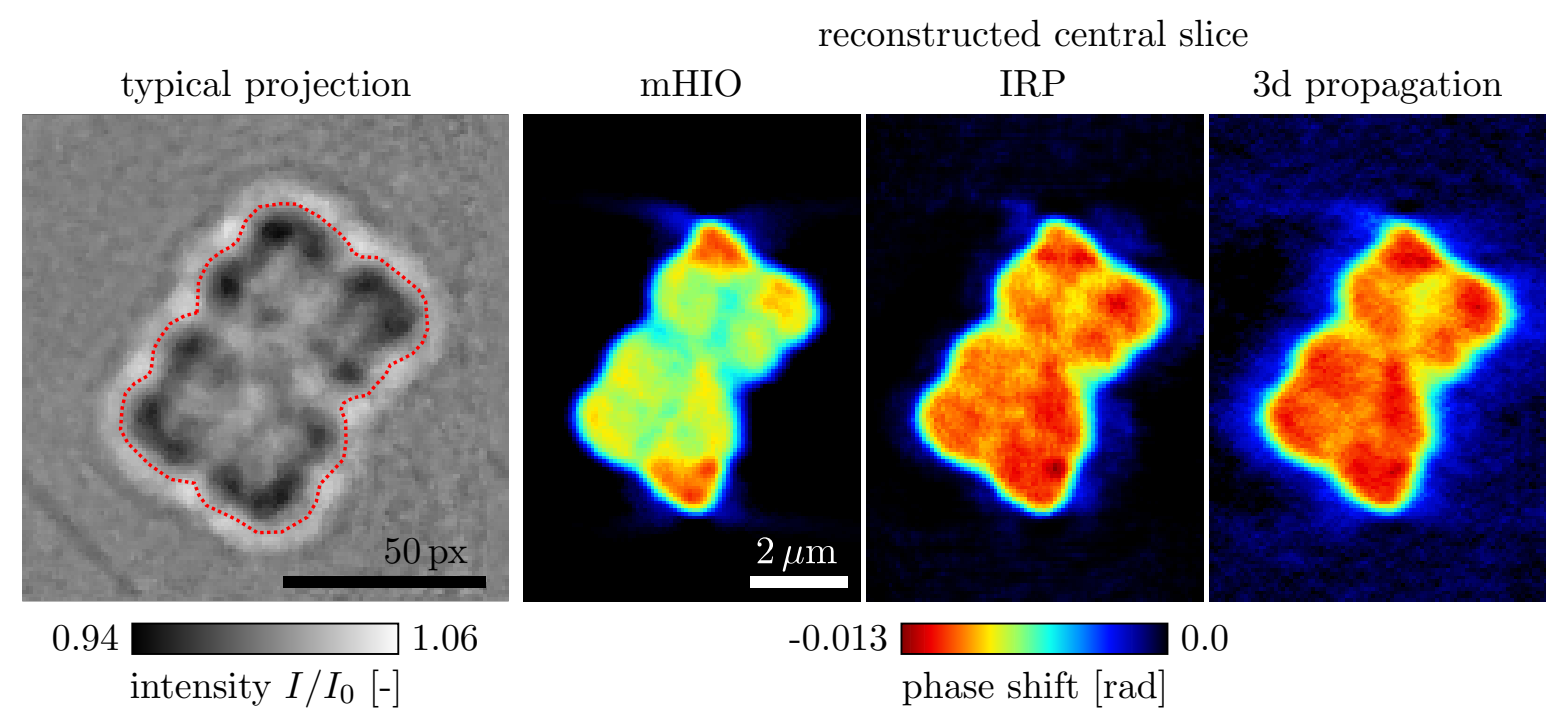

Figure 5.16: Comparison of different reconstructions from the same experimental data. Left, a holographic intensity image of the sample (freeze-dried Deinococcus radiodurans bacteria, dispersed on an ultra-thin $\mathrm{Si}_{3} \mathrm{~N}_{4}$ membrane) is shown. For the mHIO phase retrieval, a support has been derived from the data automatically during the iterations, which is indicated by the red dashed line on the hologram. Slices (1 px depth) through different reconstructions illustrate the advantage of the $3 \mathrm{~d}$ approaches. See the text for details.

3d-propagation-based reconstruction has been implemented as a modified ER scheme using only the positivity constraint for $\beta$ and $\delta$ also utilised in IRP (without soft coupling as introduced before). The result is of a similar reconstruction quality using only a fraction of the computation time.

\subsubsection{Summary and outlook}

In this chapter, two methods combining phase retrieval with tomography have been presented. The examples shown clearly demonstrate the value of tomographic consistency as a constraint in iterative phase retrieval and the advantage compared to common reconstruction schemes where phase retrieval is carried out independently for each hologram. The superior reconstruction quality can be further enhanced by incorporating 3d constraints which could not be applied previously. Masking of interior structures of the object or a sparse distribution of features in 3d are only two of many possible examples. In general, constraints can not only be formulated in physically correct and direct terms in $3 \mathrm{~d}$, but can possibly also be applied to a higher fraction of voxels.

The first of the two methods, IRP, comes with the drawback of high numerical costs compared to more traditional schemes. For the weak-object approximation, a second reconstruction scheme was developed, based on the concept of $3 \mathrm{~d}$ propagation. It inverts the usual sequence of first retrieving the phase information of all projections individually followed by an inverse Radon transform. This results in a tremendous enhance- 
ment in reconstruction speed compared to IRP, reaching a similar level as the classical schemes. In the case of many propagations, the $3 \mathrm{~d}$ propagation based approach can even outperform the classical schemes, since then a $3 \mathrm{~d}$ propagation $\left(\mathcal{O}\left(n_{y} n_{x}^{2} \log \left(n_{y} n_{x}^{2}\right)\right)\right)$ can be computed faster that $N_{\alpha} 2$ d propagations $\left(\mathcal{O}\left(N_{\alpha} n_{x} n_{y} \log \left(N_{\alpha} n_{x} n_{y}\right)\right)\right.$ with $n_{d}$ the pixel number in the corresponding dimension). At the same time, the $3 \mathrm{~d}$ propagation method preserves the essential advantages of tomographic consistency, which has been found to stabilise phase retrieval for otherwise under-determined data. Even though the $3 \mathrm{~d}$ propagation does not lead to major improvements in the quality of direct CTF phase retrieval, it can be useful to quickly investigate the influence of regularisation parameters directly in $3 \mathrm{~d}$, since the numerically expensive tomography has to be carried out only once for all parameters. Finally, like IRP, the concept can easily be generalised to several detection planes and - somewhat less straightforward - also to more complex illumination wave fields than plane waves. 



\section{Dynamic tomography of sedimenting micro-spheres}

Up to this point, we have treated every sample so far as a stable, rigid body during the measurement. However, not only are three dimensional structures of interest, but three dimensional dynamics as well. Sedimentation is a perfect example: As one of the oldest technologically employed processes, it is used for segregating particles from liquids and, within certain boundaries, also from each other [93]. It is a critical part of a number of fields, among them chemistry, biology, materials science and manufacturing, as well as pharmaceutical formulation [94]. On an industrial scale, comprehending the behaviour of paper and pulp, paints, water purification, combustion and erosion, to mention only a few, is of great interest. As such, sedimentation is one of the fundamental problems in non-equilibrium dynamics [95]. Usually, sedimentation is associated with gravity but can also be driven by electrical or other forces. Enhanced by centrifugation (then with a position-dependent force-field), it is an integral part of laboratory routine.

However, many aspects of this process are poorly understood, even for the simplest model system of identical solid spheres settling in a large container [93-99] and lead to surprising effects such as colloidal inflations and settling disasters [96] or velocity fluctuations reminiscent of turbulence, even though the Reynolds number is very low [99]. The investigation of this model system for colloidal dynamics dates back to at least 1908, when JEAN-BAPTISTE PERRIN studied the concentration profile that settling induces in suspensions of similarly sized particles. He showed sedimentation to be a powerful investigation tool, allowing, amongst others, for an accurate evaluation of Avogadro's number and the confirmation of theoretical predictions concerning diffusion, i.e. the undirected random motion of particles based on their thermal energy. The main result of PERRIN's work was the prove that in a sedimentation equilibrium, when the sedimentation of each material equals the rate of diffusive transport in the opposite direction, the distribution of monodisperse particles mirrors the barometric law for ideal gases. For this decisive confirmation of the particle nature of matter, Perrin was awarded the Nobel Prize in Physics in 1926.

Since then, macroscopic properties of sedimentation such as the mean settling velocity have been quite well understood. However, less is known about microscopic ("particle level") dynamics, like the previously mentioned velocity fluctuations [93, 95]. On average, a single particle in a fluid will settle according to the Stokes velocity

$$
v_{S}=\frac{2}{9} \frac{\Delta \varrho a^{2} g}{\eta}
$$

where frictional forces and external forces cancel [93]. Here, $\Delta \varrho=\varrho_{p}-\varrho_{f l}$ is the density difference of particle and fluid, $a$ is the particle radius, $\eta$ is the fluid viscosity and $g$ the gravitational acceleration. If the particle density is increased, settling gets affected by 
hydrodynamic interactions: In a uniformly concentrated suspension, Stokes' velocity $\left\langle v_{s}\right\rangle=f(\phi) v_{S}$ is decelerated by the hindered settling function $f(\Phi)$, depending on the concentration $\Phi$ [93]. However, as predicted by CAFLISCH and LUKE [100], small fluctuations in the particle number density already lead to a large variance in their speed: Settling spheres replace fluid which flows up, creating a highly complex system of currents interacting with the particles.

This phenomenon has been investigated in numerous experiments, theoretical as well as numerical studies (see [95] for a summary of the literature). Good agreement between theory and experiments was found in the prediction of temporal behaviour: The long-time sedimentation velocity variance was shown to be characteristic of a diffusion process [101]. Contrarily, inconsistencies arose with respect to the spatial correlations: Theoretical arguments as well as early simulations predicted a divergence of the fluctuation magnitude with the container size [95, 96], while experiments suggested independence from the system size. This could be solved in [97] using particle imaging velocimetry (PIV) to track a few marked tracer particles in a suspension of large, nonBrownian spheres in 2d: The fluctuations exhibit characteristic correlated 'swirls' with a size of about 20 mean interparticle distances. Only systems smaller than such a swirl show a dependency on the system's dimensions, while in larger systems a saturation of the fluctuations can be observed. Since such effects are not visible in the macroscopic concentration profile $[93,96]$ and are "extremely hard to tackle computationally" [95], the example shows the importance of direct experimental measurements of the particle dynamics.

In general, the dynamics of particles suspended in a fluid is given by a combination of sedimentation and diffusive Brownian motion. The specific dynamic regime of a colloidal system is characterised by the Péclet number $P e:=a v_{S} / D$ which is proportional to the ratio of ideal Stokes motion $v_{s}$ and the diffusion coefficient as defined by the Stokes-Einstein equation

$$
D:=\frac{k_{B} T}{6 \pi \eta a}
$$

for spherical particles in a liquid with low Reynolds number [94]. Here, $k_{B}$ is the Boltzmann constant and $T$ the temperature. So far, mainly for practical reasons, investigations of sedimentation have taken place predominantly in the regime of large "non-Brownian" particles [98]. The other extreme, i.e. (almost) purely diffusive Brownian motion, has been extensively studied as well, both by a wide range of techniques such as PIV, dynamic light and acoustic scattering, as well as numerous optical microscopy techniques (see [94] for a summary). However, the important regime of $P e \approx 1$ is hard to access with common setups, and when neither diffusion, nor sedimentation can be neglected, the process is intrinsically non-stationary [94]. Recently, a study based on X-ray photon correlation spectroscopy has been released, helping to overcome several limitations of optical systems (e.g. the need for optical index-matching and low particle densities) and allowing to measure regimes with $P e<1$ [98]. However, a complete characterisation of the dynamics in the capillary, including the evolution of complex fluid currents, wall effects and processes at the sediment growth interface carefully avoided in the experiments mentioned so far, is only possible by full access to the $3 \mathrm{~d}$ trajectories of a large number of particles. 
As an emerging field, time-resolved (X-ray) computed tomography (CT) holds unique potential for non-invasive analysis of such processes, combining high spatial with temporal resolution. Novel experimental capabilities have been opened up by highly brilliant synchrotron sources, new contrast mechanisms including phase contrast, as well as faster detectors and advancements in data processing. However, all numerical reconstruction methods mentioned so far depend on the consistency of a static object which is clearly violated by motion. The importance of this premise can be well observed by the severe artefacts caused by global drifts or vibrations during measurement, occurring even without any internal changes of the object itself. Fortunately, certain consistency demands can be used to correct for global motion artefacts, even if this can be a complicated task covering all degrees of freedom. Methods include registration of the projections to their similar neighbours or to a 3d guess of the object $[102,103]$. However, if the sample structure or density itself changes during the measurement, tomographic consistency is lost and all methods depending on this property must fail, from simple alignment procedures to tomographic reconstruction (FBP, ART) and methods of iterative sinogram retrieval allowing for sparse sampling. Thus, movements during recording have been identified as a major source of artefacts but cannot be completely avoided. Examples range from the body motion (heartbeat, breathing) of patients in clinical CT to radiation-induced structural changes in micro-CT like sample degradation or the formation of bubbles in embedding media.

The intentional measurement of such dynamics requires improved procedures. In this context, methods allowing to view the process from multiple perspectives are of significant interest. If multiple sources and detectors are available, measurements can be carried out at the same time, similar to 3d PIV, capturing the motion of a large number of markers in a current. An alternative is to acquire a rapid series of projections with large angular increment. This is for example exploited in high speed magnetic resonance tomography (MRT), where the projection angles can be freely chosen by selection of the magnetic force gradients [104].

Contrarily, micro-CT is typically based on a single X-ray source, which cannot be rotated freely (synchrotron). Hence, the object has to be rotated, allowing the recording of consecutive projections only in comparatively small angular steps as limited by the rotation speed of the sample. This was the starting point for the development of several approaches to $4 \mathrm{~d}$ tomography, including optimised weighting schemes, corrections for affine motion, local time-stationarity hypothesis, and periodicity hypothesis (see [105] for a review). Notably, for the special case of cyclic processes such as a beating heart or a breathing lung, acquisitions can be gated or triggered to cover different phases of the motion [105-107]. If all the projections are captured at the same state of the motion or even a whole cycle is recorded for every angle, static solutions can be generated for the different time points within one cycle. Hardware gating as well as software gating (a posteriori) have both seen convincing implementations, unravelling complex spatiotemporal processes such as the muscle movement during an insect flight $[108,109]$. For general motions, however, the common approach is to minimise the influence of sample dynamics by increasing the recording speed and treating the sample as quasistatic during the acquisition time of each tomographic set. The minimum time required for a complete scan is influenced by multiple factors: photon flux, camera efficiency and repetition rate, as well as the rotation speed. Due to radiation damage, even if 
theoretically possible, the flux on the sample cannot be increased without limit in order to cut down the acquisition time. Furthermore, the rotation speed cannot be increased arbitrarily, as a single image should not be blurred by rotation. Hence, the recording of the full $180^{\circ}$ range will often be too slow to justify the assumption of static conditions in tomography.

In this chapter, a proof-of-principle experiment is presented recording the fast dynamics of sedimenting $\mathrm{SiO}_{2}$ micro-spheres in a water-filled capillary at a Péclet number of 14. To this end, we first estimate the errors caused by motion in a reconstruction derived for the quasi-static assumption and show what can nevertheless be achieved if the problem is reduced from resolving a complex sample structure to determining the position of simple markers in space and time, based on a set of tomographic projections. Subsequently, the experiment is described. The conventional sequential approach of phase-retrieval followed by tomographic reconstruction is used to obtain the trajectories of several thousand particles in parallel. It shows the limits but also the possibilities of fast conventional tomography and offers an exciting insight into otherwise hidden processes. If the glass spheres are considered as markers, the results can be easily transferred to other suitable samples. A special feature of the setup is the use of $\mathrm{X}$-ray waveguide (WG) illumination. This is challenging, since the low photon flux emanating from the WG prohibits short acquisition times and consequently impedes a fast rotation and the investigation of rapid processes. On the other hand, this type of setup offers high resolution X-ray microscopy with geometrical magnification and high sensitivity due to phase contrast, which is perfectly suited for the investigation of small and weakly interacting samples.

\subsection{Motion artefacts and positioning error}

To illustrate the problem of local inconsistency arising in dynamic tomography, Fig. 6.1 shows the most basic kind of motion: In the acquisition time of the sinogram, separated markers without any inner structure move with constant speed along straight paths. We additionally assume that the exposure time of each projection is short enough that no motion blurring occurs in the single projections. Sinograms have been simulated for different angular ranges, as indicated in the figure. The reconstructions performed by a filtered backprojection do not show the marker averaged on its actual path but on arcs and half-circles tangentially touching the original trajectory, as indicated in the first example where the start and end positions have been highlighted. The paths have enhanced edges with negative values. This clearly indicates inconsistency since the projections are strictly positive. Negative values are introduced by the ramp-filter to compensate for the PSF of static tomography and cannot superpose at the right positions here. Additionally, streaks occur in the direction of the first and last projections of the sinograms, which display the largest temporal and structural difference, as indicated by the red dashed lines in Fig. 6.1. This direction has highest coverage by the set of all projections. Importantly, the appearance of the artefacts and the curvy paths of the markers do not depend on the position, i.e. the distance from the centre of rotation, but only on speed and direction relative to the direction of the projections. 


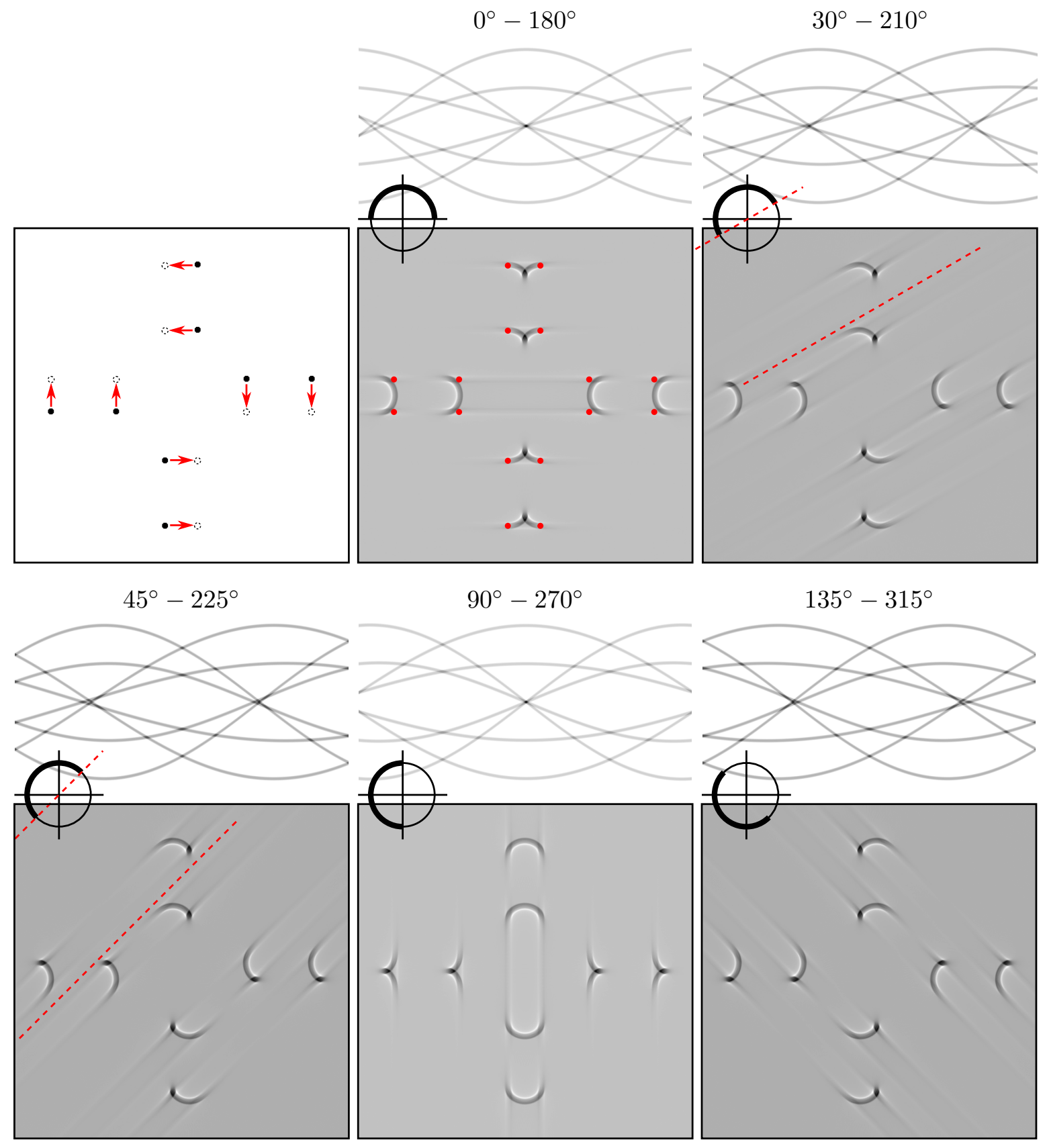

Figure 6.1: Artefacts arising from particles moving along a straight path with constant velocity during the tomographic measurement. Depending on the angular coverage of the projections, different sinograms are obtained from the process sketched in the top-left image. The FBP reconstructions show curved particle traces with emphasised edges and streaks along the first and last projection direction.

As can clearly be seen in Fig. 6.2 for the same angular coverage as before, a higher velocity only scales the path but does not result in a change of characteristic with respect to the artefacts. 


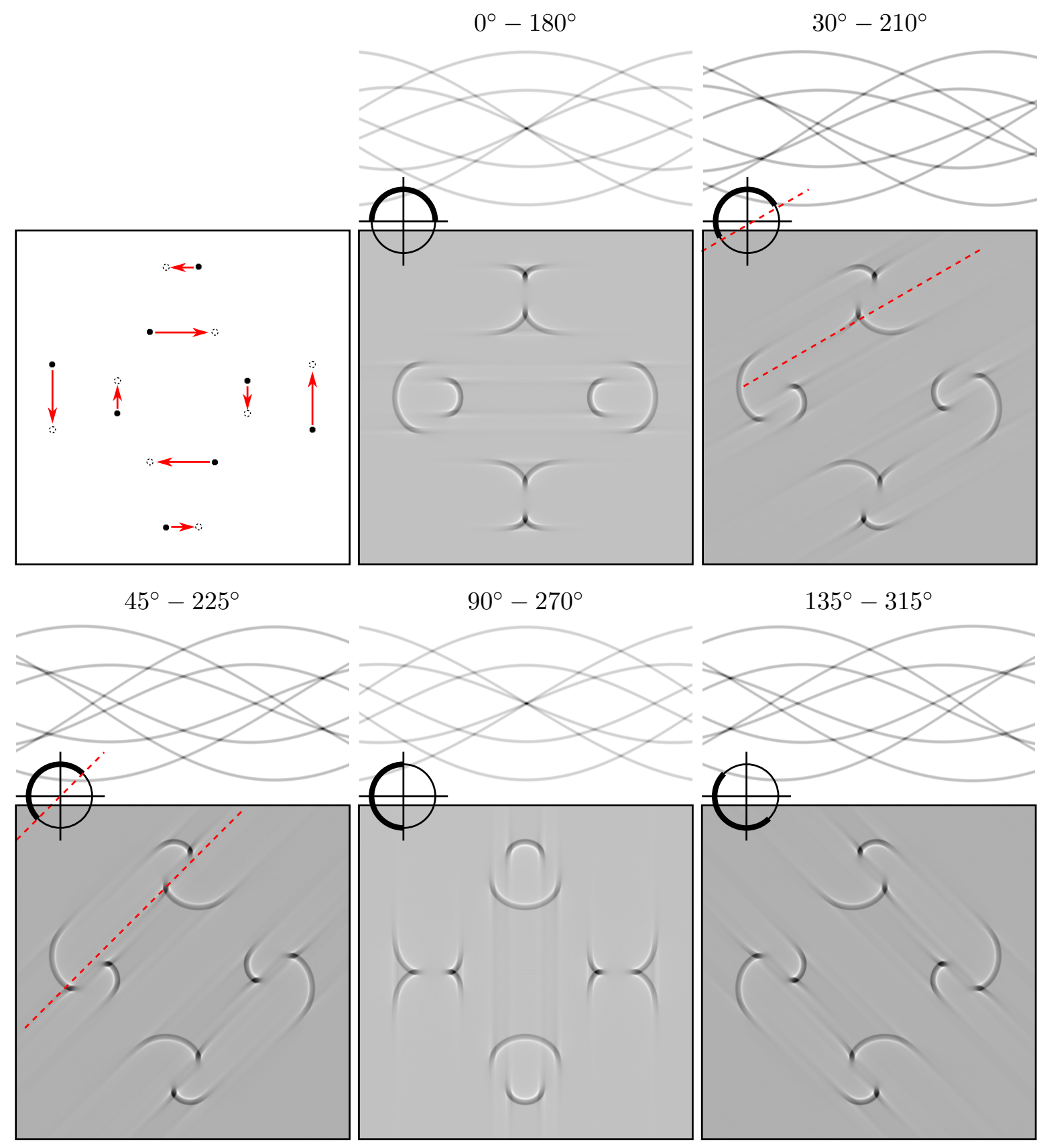

Figure 6.2: The shape of the motion artefacts does not depend on the position of the features but only on their velocity relative to the directions of the projections.

For a more quantitative treatment, below, only the path $\vec{r}(t)$ of a moving particle is considered, but not the reconstruction of the sample structure. A point moving in a fixed $x-z$ coordinate system perpendicular to the axis of rotation serves as the most simple model system. The situation is sketched in Fig. 6.3. Let us first consider the case of a resting particle positioned at $\vec{r}_{0}=(x, z)$. The first projection along the $z$-axis at time $t=0$ yields the value $s_{0}$. One time step later, at $t=\Delta_{t}$, the coordinate system has been rotated by $\alpha$, resulting in the value $s_{t}$ for the projection along the $z^{\prime}$-axis. In 


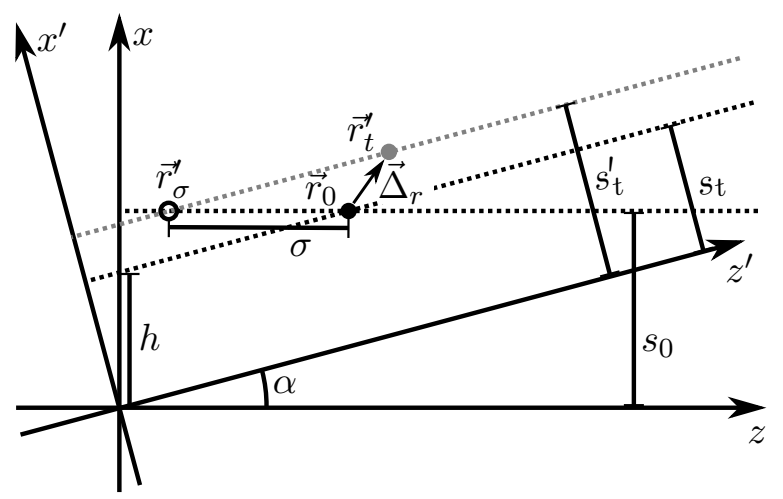

Figure 6.3: Sketch of two subsequent projections of a moving feature.

conventional tomography, the position $\vec{r}_{0}$ can be calculated as the point of intersection of the backprojected signals $s_{0}$ and $s_{t}$ (dashed lines in Fig. 6.3) as follows. $x=s_{0}$ was measured directly, with $h=s_{t} / \cos (\alpha)$ one calculates the second coordinate as

$$
\begin{aligned}
s_{0} & \stackrel{!}{=} \frac{s_{t}}{\cos \alpha}+z \tan \alpha \\
\Rightarrow z & =\frac{s_{0}}{\tan \alpha}-\frac{s_{t}}{\sin \alpha} .
\end{aligned}
$$

However, we are interested in the case of a dynamic particle. If the point moves from $\vec{r}_{0}$ to $\vec{r}_{t}^{\prime}=\vec{r}_{0}+\vec{\Delta}_{r}$ between the two recordings, the second measurement will yield the value $s_{t}^{\prime}$ instead of $s_{t}$. The point of intersection of the backprojected signals $s_{0}$ and $s_{t}^{\prime}$ then is $\vec{r}_{\sigma}$, as shown in Fig. 6.3. Thus, if one naively replaces $s_{t}$ by $s_{t}^{\prime}$ in Eq. (6.3), the calculated $z$-component belongs to the point $\vec{r}_{\sigma}$, resulting in a positioning error

$$
\begin{aligned}
\sigma & =\left|\vec{r}_{0}-\vec{r}_{\sigma}\right|=z-z_{\sigma} \\
& =z-z_{\sigma}=\left[\frac{s_{0}}{\tan \alpha}-\frac{s_{t}}{\sin \alpha}\right]-\left[\frac{s_{0}}{\tan \alpha}-\frac{s_{t}^{\prime}}{\sin \alpha}\right]=\frac{s_{t}^{\prime}-s_{t}}{\sin \alpha} .
\end{aligned}
$$

In order to relate this positioning error to the initial position $\vec{r}_{0}$ and the displacement $\vec{\Delta}_{r}=\left(\Delta_{x}, \Delta_{z}\right)$, consider that

$$
\begin{aligned}
s_{t} & =x \cos \alpha+z \sin \alpha \\
s_{t}^{\prime} & =\left(x+\Delta_{x}\right) \cos \alpha+\left(z+\Delta_{z}\right) \sin \alpha \\
\Rightarrow \Delta_{s_{t}} & \equiv s_{t}^{\prime}-s_{t}=\Delta_{x} \cos \alpha+\Delta_{z} \sin \alpha .
\end{aligned}
$$

Thus, we arrive at

$$
\sigma\left(\vec{\Delta}_{r}, \alpha\right)=\frac{\Delta_{s_{t}}}{\sin \alpha}=\Delta_{z}+\frac{\Delta_{x}}{\tan \alpha}
$$

Note that $\sigma$ only depends on the displacement vector $\vec{\Delta}_{r}$ and the angle increment $\alpha$, not on the initial position $\vec{r}_{0}$, confirming this previous observation. For typical small $\alpha$, the deviation depends mainly on $\Delta_{x}$, the motion perpendicular to the direction of the projection. For a step with length $\left|\Delta_{r}\right|$ in the direction of an arbitrary angle $\gamma$ 
with respect to the $z$-axis, it is $\sigma(\alpha, \gamma)=\left|\Delta_{r}\right|(\cos (\gamma)+\sin (\gamma) / \tan (\alpha))$. For evenly distributed $\gamma$, the mean error $\langle\sigma\rangle_{t}$ of the determination of position is given by

$$
\begin{aligned}
\langle\sigma(\alpha)\rangle & =\frac{\left|\Delta_{r}\right|}{2 \pi} \int_{0}^{2 \pi}\left|\cos \gamma+\frac{\sin \gamma}{\tan \alpha}\right| \mathrm{d} \gamma=\frac{2\left|\Delta_{r}\right|}{\pi} \sqrt{1+\tan ^{-2} \alpha} \\
& =\frac{2\left|\Delta_{r}\right|}{\pi|\sin \alpha|} .
\end{aligned}
$$

Better results can be expected when the motion of the point is taken into account. Let $\vec{r}_{0}=(x, z)$ be the known initial point of a particle at $t=0$. In the simplest case of a piecewise constant velocity $\Delta_{r}=\left(\Delta_{x}, \Delta_{z}\right)=$ const., the projections

$$
\begin{aligned}
s_{t}^{\prime} & =\left(x+\Delta_{x}\right) \cos \alpha+\left(z+\Delta_{z}\right) \sin \alpha=s_{t}+\Delta_{s_{t}} \\
s_{2 t}^{\prime} & =\left(x+2 \Delta_{x}\right) \cos 2 \alpha+\left(z+2 \Delta_{z}\right) \sin 2 \alpha=s_{2 t}+\Delta_{s_{2 t}}
\end{aligned}
$$

are measured at the times $t=\Delta_{t}$ and $2 \Delta_{t}$. Since $x, z$, and $\alpha$ are known, the system of equations can be solved for $\Delta_{x}$ and $\Delta_{z}$, yielding

$$
\begin{array}{r}
\Delta_{x}=2 \cos \alpha\left[s_{t}^{\prime}-s_{t}\right]-\frac{s_{2 t}^{\prime}-s_{2 t}}{2} \\
\Delta_{z}=\left[s_{t}^{\prime}-s_{t}\right] \frac{1-2 \cos ^{2} \alpha}{\sin \alpha}-\frac{s_{2 t}^{\prime}-s_{2 t}}{2 \tan \alpha} .
\end{array}
$$

With this, the desired $\vec{r}_{t}=\vec{r}_{0}+\vec{\Delta}_{r}$ can be found. If the true velocity in the time interval $\left[\Delta_{t}, 2 \Delta_{t}\right]$ is $\vec{\Delta}_{r}+\vec{\Delta}_{r}^{\prime}$, the mean positioning error is

$$
\left\langle\sigma_{v}(\alpha)\right\rangle=\frac{\Delta_{r}^{\prime}}{\pi \sin \alpha}
$$

analogous to Eq. (6.6). Since the assumption of a constant velocity $\left(\Delta_{r}^{\prime} \ll 1\right)$ in the time interval $2 \Delta_{t}$ is usually describing the dynamics much better than the assumption of a constant position $\left(\Delta_{r} \ll 1\right)$ in the interval $\Delta_{t}$ (de facto excluding dynamics), this drastically improves the reconstruction quality. However, the initial position has to be known. It can either be obtained directly from two additional projections (at $t=0$ and $t=3 \Delta_{t}$, requiring the assumption of a constant velocity to hold for three time steps) or it can be derived indirectly by the following procedure: Since the reconstructed path of a particle tends to converge to the actual path even with an incorrect initial position ${ }^{1}$, many reconstructions with varying guesses for $\vec{r}_{0}$ can be computed. Paths with the same end-point are then considered as converged, and the required information has been found (invert time for the reconstruction of the original path from a known point).

In summary, the accuracy of the reconstructed paths can be improved drastically if the possibility of motion is included in the reconstruction method. However, the example discussed here fulfils two requirements: First, the marker can be identified already in the projections, requiring a small number of markers or known, unique sample features often not present in tomography which is carried out in order to estimate the sample's

\footnotetext{
$\overline{1}$ Result from simulations, data not shown.
} 

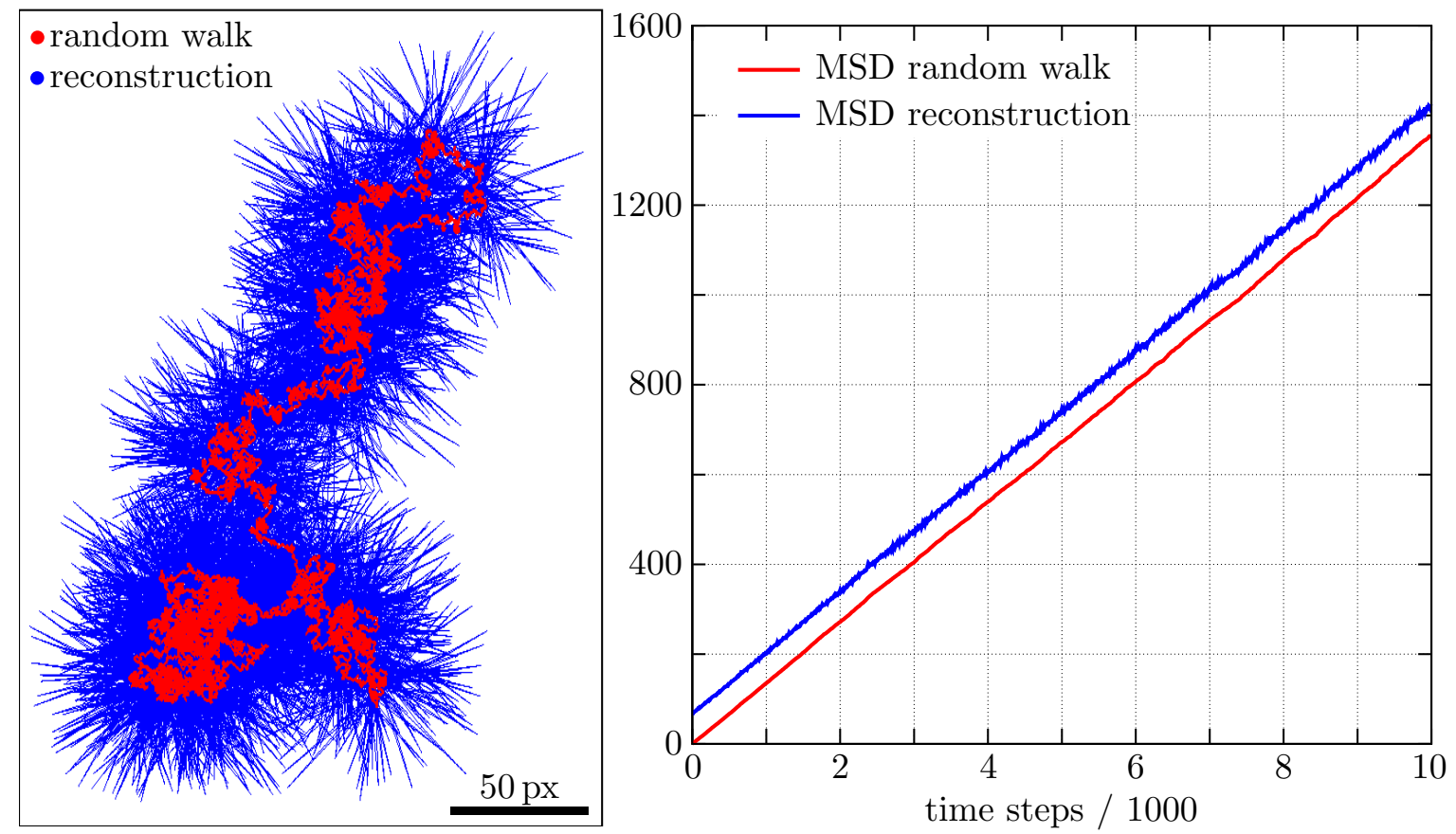

Figure 6.4: A discrete random walk with a length of 10000 steps (red) was captured in a sinogram with 50 full rotations. The direct reconstruction (see text for details) reveals the heavily disturbed path drawn in blue. Despite the large systematic errors, the MSD of the reconstruction shows a line with in the same slope $(\propto D)$ but an offset compared to the original trajectory.

structure at all. Second, the marker's positions (or their n-th derivatives) are assumed to be uniform over the time steps required for measurement. Clearly, incorporating higher orders of the motion further improves the results but the corrections do not apply to arbitrary rapid changes in particle speed which occur e.g. in diffusion. But also this stochastical process can be elucidated by tomography even without any correction. For random Brownian motion of particles in a fluid, the characteristic diffusion coefficient $D$ is related to the 3 d mean squared displacement (MSD) by

$$
\left\langle\left|\vec{r}(t)-\vec{r}_{0}\right|^{2}\right\rangle=6 D t
$$

The MSD can be derived from a sinogram as illustrated in Fig. 6.4 on the example of a simulated discrete $2 \mathrm{~d}$ random walk. For each of the $10^{4}$ time-steps, the next particle position on a pixel grid was selected randomly from the actual position and the 8 neighbouring pixels. Each step of the motion was projected along the $z$-axis rotating with a velocity of $1.8^{\circ} / \Delta_{t}$, covering the process in 50 full rotations. The direct reconstruction of the marker's position from the projections with Eq. (6.3) yields the dramatically disturbed path shown in Fig. 6.4. Nevertheless, the MSD as calculated from this reconstruction by

$$
\operatorname{MSD}(t) \equiv\left\langle\left|\vec{r}(t)-\vec{r}_{0}\right|^{2}\right\rangle=\frac{1}{N} \sum_{n=1}^{N}\left(\vec{r}\left(t_{n}+t\right)-\vec{r}\left(t_{n}\right)\right)^{2}
$$


with the number of sections $N$ of the path with same length $t$ shows the same slope $\propto D$ as the ideal path, only with an offset $\left|\Delta_{\vec{r}}\right|^{2}$ due to the large but systematically positioning errors. In this simulation, again the diffusing marker could be identified in the projections. In the following sections, an experimental situation is described where this is not possible anymore since the density of moving particles is too high. Moreover, the goal was not to obtain a statistical mean describing the overall motion but the actual path of the particles, considering the described challenges.

\subsection{The sedimenting spheres experiment}

An experiment demonstrating the possibilities of high (spatial) resolution dynamic Xray tomography for randomly moving particles was carried out in May 2016 at the multi-purpose "Göttingen instrument for nanoscale imaging with X-rays" (GINIX) setup at the P10 coherence beamline of the PETRA III storage ring at the Deutsches Elektonen Synchrotron (DESY) in Hamburg [110, 111]. $\mathrm{SiO}_{2}$ micro-spheres sedimenting in a small, water filled glass capillary were used as a sample. A unique feature of the setup is the usage of X-ray waveguides. With the drawback of a drastically reduced photon flux which is challenging for fast image acquisition and dynamic tomography, they enable highly sensitive holographic near-field measurements of weak objects like biological tissue with high resolution. In this chapter, it is shown that time-resolved tomography with a temporal resolution on the order of considerably less than a second and a spatial resolution on the order of $0.1 \mu \mathrm{m}$ is possible under such experimental conditions, opening the path to study dynamic processes in a natural $3 \mathrm{~d}$ environment. The most important experimental parameters are summarised in the table below and explained in detail in the following sections.

\begin{tabular}{ll}
\hline Sample & $2 \mu \mathrm{m} \mathrm{SiO}_{2}$ spheres sedimenting in \\
& $\mathrm{H}_{2} \mathrm{O}$ filled capillary \\
Photon energy & $8 \mathrm{keV}$ \\
Fresnel number & $F_{1} \approx 2.1 \cdot 10^{-3}$ \\
Eff. pixel size & $\approx 116 \mathrm{~nm}$ \\
Acquisition frame rate & $\leq 143 \mathrm{FPS}$ \\
Rotation speed & $\leq 1 \mathrm{~Hz}(\mathrm{up}$ to 2 sinograms $/ \mathrm{s})$ \\
Covered process time & $39.9 \mathrm{~s}$ \\
\hline
\end{tabular}

\subsubsection{P10 beamline setup}

The GINIX setup is sketched in Fig. 6.5. An undulator in the storage ring PETRA III serves as the photon source, followed by a cryogenic cooled double crystal $\mathrm{Si}(111)$ 

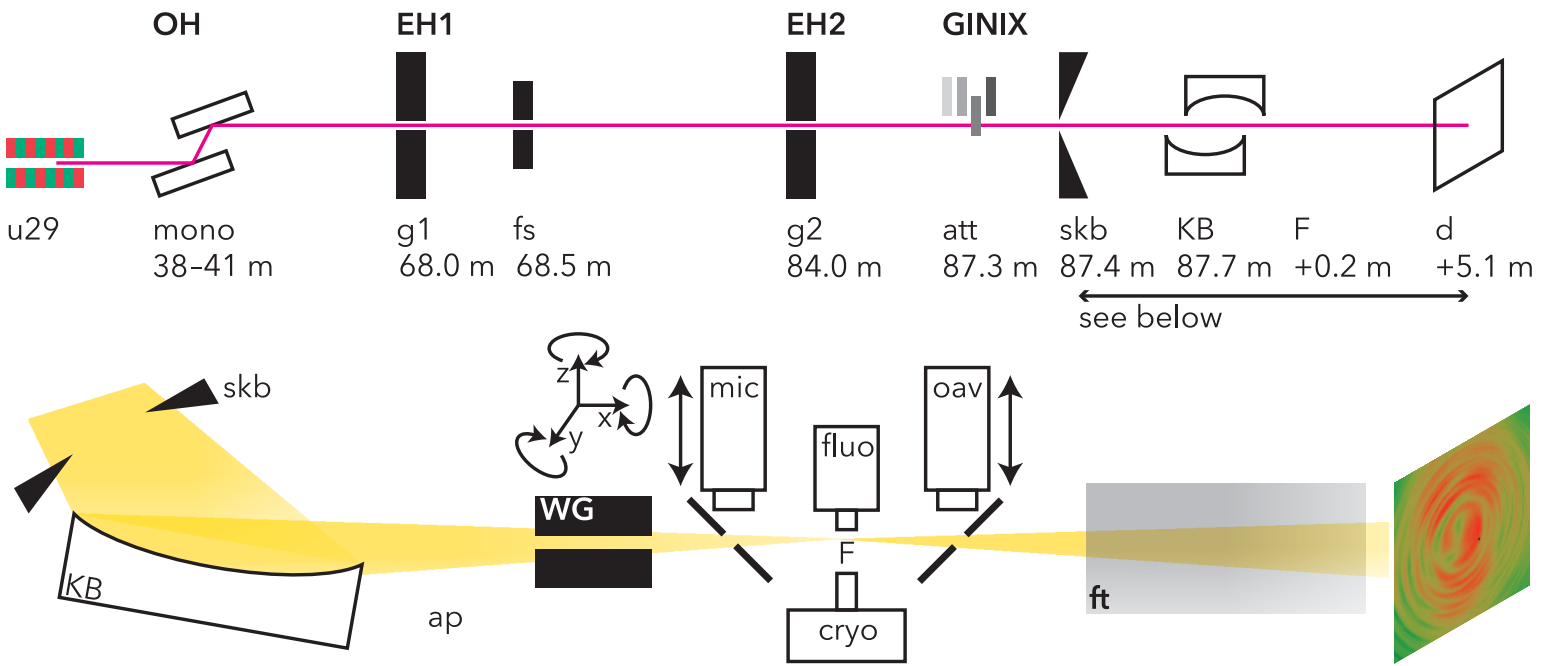

$\mathrm{ft}$

Figure 6.5: Top: Schematic of the P10 beamline and the GINIX endstation, with undulator (u29), monochromator (mono), slits (g1, g2, skb), fast shutter (fs), attenuators (att), KB mirrors, focal spot $(\mathrm{F})$ and detection plane (d) with the distances measured from the undulator. Bottom: Detailed GINIX endstation with aperture (ap), waveguide (WG), on-axis optical microscopes (mic, oav), optional fluorescence detector (fluo), optional cryojet sample environment (cryo), and the flight tube (ft). Image obtained from [111].

monochromator with a resolving power of $0.01 \%$. The energy can be tuned in the ranges from $3.8 \mathrm{keV}$ to $10.5 \mathrm{keV}$ and $11.4 \mathrm{keV}$ to $25.0 \mathrm{keV}$. At a distance of about $84 \mathrm{~m}$ behind the undulator, the beam size is defined by horizontal and vertical slits. The centrepiece of the P10 endstation is a pair of Kirkpatrick-Baez mirrors (KB) installed in a vacuum chamber located $87.4 \mathrm{~m}$ behind the undulator, focusing the beam to a spot outside the chamber with a size down to $200 \mathrm{~nm} \times 200 \mathrm{~nm}$ and a total coherent flux of up to $10^{11} \mathrm{ph} / \mathrm{s}$. In the focus, a waveguide can be placed, acting as virtual source of a clean and coherent wave field illuminating the sample placed on a motorised air-bearing rotation stage. The adjustment is facilitated by remote-controlled optical microscopes, most importantly the 'on axis view' (OAV) with a hole drilled through the optics, allowing the x-rays to pass largely unperturbed. While waveguide and sample are freely accessible under ambient pressure, the beam enters an evacuated flight tube shortly behind the rotation stage to prevent signal absorption and air scattering on its way to the detector which is placed directly behind the vacuum tube about $5.1 \mathrm{~m}$ downstream of the sample. Due to the GINIX optics, the optimum photon energy range is $6 \mathrm{keV}$ to $14 \mathrm{keV}$. See $[110,111]$ for detailed descriptions of the setup including attenuators, additional sample stages, apertures, beamstops and the optional cryogenic sample environment.

Apart from the illumination, which is detailed below, the rotation stage and the detector are crucial components for fast tomography. GINIX is equipped with an UPR-160 ultraprecision rotation stage (Physik Instrumente (PI) GmbH \& Co. KG) with air bearings for improved flatness and a velocity of up to $360^{\circ} / \mathrm{s}$. However, the motor controller only allowed for a limited travel range of about $-3600^{\circ}$ to $3600^{\circ}$, corresponding to an 
uninterrupted measurement time of $20 \mathrm{~s}$ with highest rotation frequency. The stage sits on a sample tower which allows to remotely adjust the axis of rotation precisely in all directions $(x, y, z)$ and to correct for the angle around the (optical-) $z$-axis (roll) and around the $x$-axis. In the conventional setting, a second $x-y$ - $z$-positioning system sits above the stage to move the sample into (and relative to) the rotation axis. However, for many rotations the cables running through a recess in the centre of the rotation stage would wind up and eventually break, while tensions would move the sample. Thus, all motors above the rotation stage were replaced by a manually adjustable $\mathrm{x}-\mathrm{y}$ dish with micrometre screws.

The most suitable detector available for our purpose was an sCMOS camera based on model C12849-102U (Hamamatsu Photonics) equipped with a $20 \mu \mathrm{m}$ thick LuAG:Ce ${ }^{2}$ scintillator (CRYTUR, spol. s r.o.) instead of the default $20 \mu \mathrm{m}$ Gadox ${ }^{3}$ crystal, improving the decay time. All other specifications correspond to those of the C12849$102 \mathrm{U}$ [112]. The effective number of pixels is $2048 \times 2048$ covering an active area of $13.312 \times 13.312 \mathrm{~mm}^{2}$ with a pixel size of $6.5 \times 6.5 \mathrm{\mu m}^{2}$. The readout speed with a depth of $16 \mathrm{bit} / \mathrm{px}$ is 30 frames per second (FPS) in full field and up to 25655 FPS with internal pixel binning $(2 \times 2$ and $4 \times 4$ binning available $)$ and selection of a suitable region of interest (ROI). The exposure time can be selected in steps of $1 \mathrm{~ms}$ from a minimum of $1 \mathrm{~ms}$ up to $30 \mathrm{~s}$. The images were buffered on a high-speed solid-state drive (SSD) enabling a recording time of up to several hours with highest frame rate.

\subsubsection{X-ray waveguide illumination}

In the previous chapters, a clean and constant illumination has been assumed, allowing to divide the detector images by the amplitude $I_{0} \propto\left|\Psi_{0}\right|^{2}$ (see e.g. the holographic or CTF reconstruction formula). However, in practice the illumination shows distinct artefacts mainly introduced by the $\mathrm{KB}$ mirrors due to smallest deviations from the perfect elliptic shape which become visible due to the gracing incidence of radiation necessary for total reflection. A typical detector image of a KB illumination is depicted in Fig. 6.6 (a). The adjacent panel (b) shows an exemplary detector image of a $10 \mu \mathrm{m}$ diameter polystyrene sphere sample, still dominated by the illumination shape. The socalled flat-field correction, i.e. dividing image (b) by the flat field (also termed 'empty beam') depicted in (a), reveals the hologram shown in (c). There, the pattern is much better recognisable as the typical hologram of a sphere, but remaining artefacts from the illumination are apparent, spoiling the phase retrieval.

The underlying problem is that for the flat-field correction, intensities in the detection plane instead of the complex-valued wave-fields in the exit-plane are divided. This phenomenon has been studied in [113], where a numerical phase-retrieval approach for the illumination (termed probe in this context) is presented based on the small differences of flat-fields recorded at different KB-to-detector distances. In contrast, this work relies on X-ray waveguides (WG) providing a hardware solution to the emptybeam problem. To this end, Fig. 6.7 shows the detector image at the same distance as before with a $2 \mathrm{~d}$ WG adjusted in the KB focus. The illumination artefacts are clearly

\footnotetext{
${ }^{2}$ Lutetium aluminium garnet doped with cerium

${ }^{3} \mathrm{Gd}_{2} \mathrm{O}_{2} \mathrm{~S}$
} 


\subsection{The sedimenting spheres experiment}

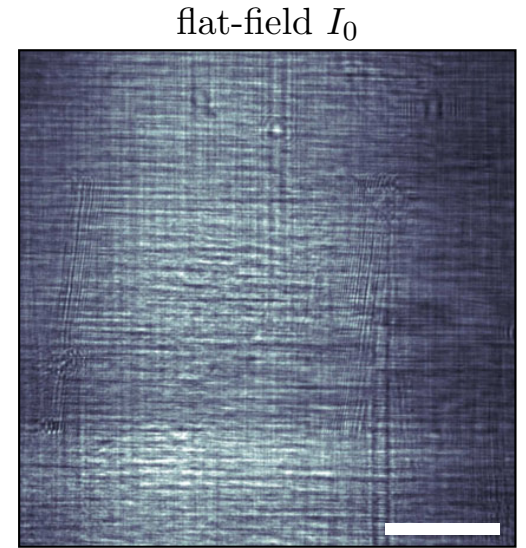

0.1

intensity [a.u.]
2.5

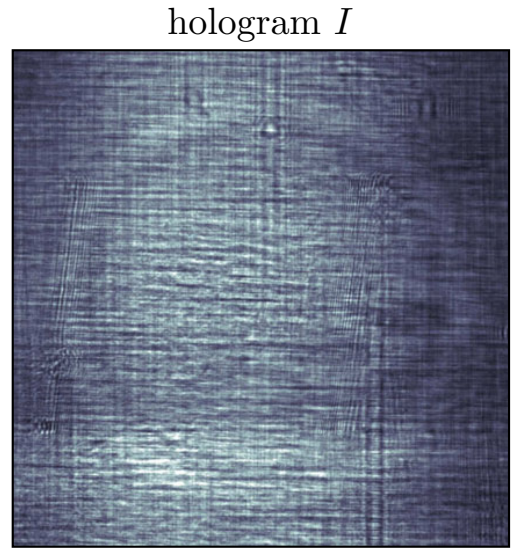

0.1

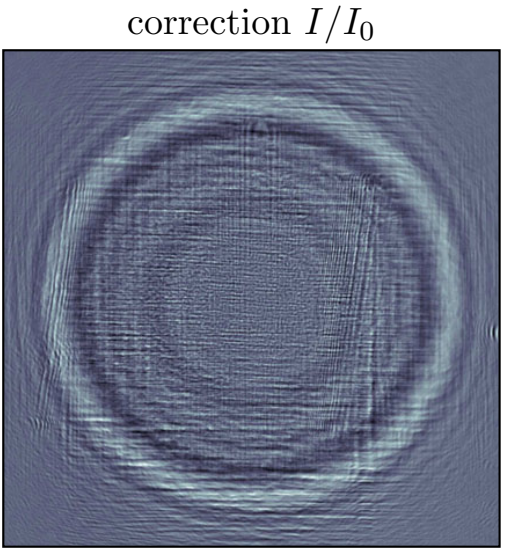

0.74

1.34

intensity [a.u.]

nomalised intensity [a.u.]

Figure 6.6: Artefacts of the so-called flat field correction. A hologram of a $10 \mu \mathrm{m}$ diameter polystyrene sphere serves as an example, recorded at beamline ID22NI (ESRF, Grenoble) with a photon energy of $20 \mathrm{keV}$. The division of the hologram with the impure KB illumination still contains artefacts which have the same shape as the illumination. The common scale bar is $4 \mu \mathrm{m}$. Data obtained from [113]

removed but note that the intensity has been decreased by a factor of 3700 in this comparison. However, since this clean illumination enables the exploitation of phase contrast, the loss of photons is compensated to a certain degree. The fabrication of WGs hinted in the figure is detailed in [114-116]. Here, we used a 1d WG mainly smoothing the illumination along the narrow, wave-guiding direction with the advantage of much higher photon flux compared to a 2d WG. A typical flat-field image is depicted in Fig. 6.9 . 
(a)

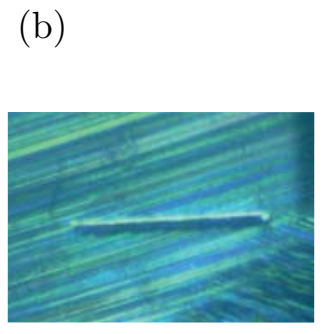

(c)

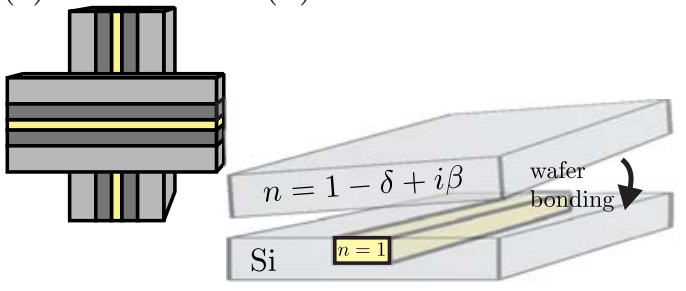

(e)

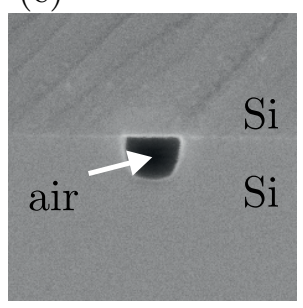

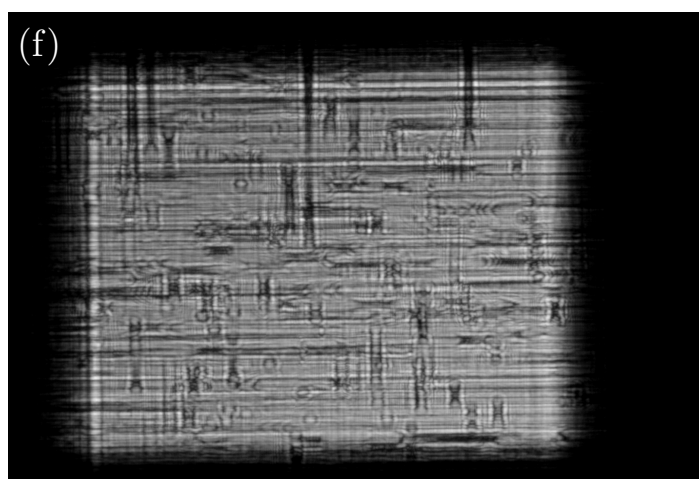

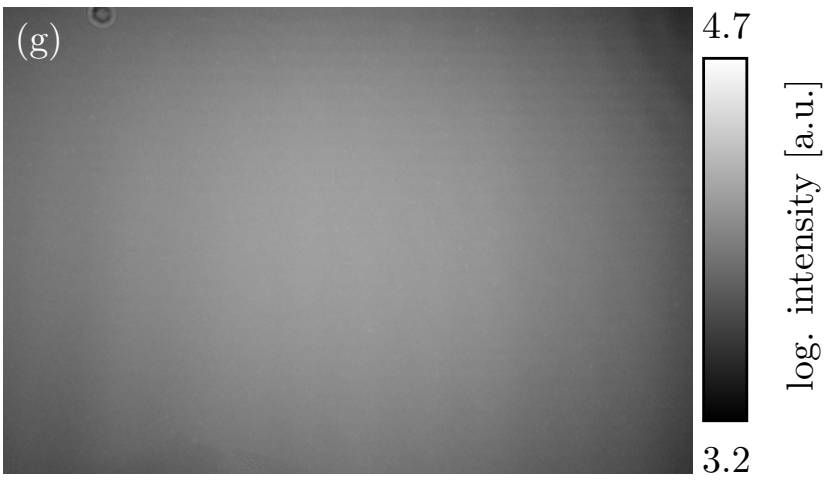

Figure 6.7: Waveguide types and cleaning effect. (a) shows an electron micrograph of a sputtered thin-film sequence $\mathrm{Ge} / \mathrm{Mo} / \mathrm{C} / \mathrm{Mo} / \mathrm{Ge}$ used for a planar waveguide system $(1 \mathrm{~d}$ WG). In (b), the relevant coupling region for the beam polished by a focused ion beam is depicted as seen in the on-axis optical microscope of the GINIX setup. By crossing two 1d WG slices, a two-dimensional waveguide system (2d WG) can be realised (c). Alternatively, 2d WGs can be manufactured by lithographic development of a waveguide channel (air) capped by wafer bonding (d). A corresponding electron micrograph is shown in (e). The effect of a $2 \mathrm{~d}$ WG can be seen in (g), smoothening the KB illumination otherwise resulting in a detector image as depicted in (f). Note, that the signal in (g) has been enhanced by a factor of about 3700 for this comparison. Images obtained from [111] (top row) and [90] (bottom row).

\subsubsection{Choice of sample}

The choice of sample depends on many constraints. The predefined parameters of the beamline were (i) a photon energy of $8 \mathrm{keV}$ due to other experiments in alternating shifts, (ii) the rotation stage limited to 36 full rotations with up to $1 \mathrm{~Hz}$, and (iii) the minimum exposure time, maximum frame rate, sensor area and position of the detector as summarised above. Contrast and magnification could be adjusted by the $z$-position of the sample in the cone-beam geometry. Placed at a region where the Fresnel number indicates high phase contrast, the lateral size of the sample is limited by the field-of-view (FOV) of the detector. Additionally, even for the fastest rotation a feature on the edge of the sample should not be motion-blurred too strongly during the exposure time of a single image, restricting sample diameter as well as feature size. The minimum exposure time itself depends on the illumination brightness and the holographic contrast enhancement, as well as on the hardware acquisition rate for a given ROI and binning (FOV and resolution, respectively). Moreover, the sample features are not allowed to move too fast in the direction perpendicular to the rotation 
axis whereas a higher velocity can be accepted along the rotation axis where the position can be determined more easily. Clearly, the motion should not be too slow, too, since we want to reach the limits of conventional tomography. As shown before, the positioning of sparse markers which are already distinguishable in the projections is a comparatively straight-forward task. In order to exploit the strengths of tomography, the marker density should be high.

All these constraints are met by glass spheres sedimenting in a capillary, in particular since concentration, size and velocity can be adjusted in a wide range. We used a quadratic cross section borosilicate glass capillary with an inner edge length of $50 \mu \mathrm{m}$ and a wall thickness of $25 \mu \mathrm{m}$ with tolerances of $\pm 10 \%$ (VitroCom Inc.). The main advantage of this shape is that the orientation of the capillary is encoded in each hologram. This is necessary since no encoder values for the rotation angle are available as common in static tomography (detector and rotation were used completely independent from each other). The maximum diameter of $\sqrt{2} \cdot 50 \mu \mathrm{m} \approx 71 \mu \mathrm{m}$ adjusted to fit the detector FOV yields a minimum effective pixel size of about $35 \mathrm{~nm}(70 \mathrm{~nm}$ with $2 \times 2$ binning for fast detector readout), corresponding to a magnification of 187 and an effective Fresnel number of $F_{1} \approx 2.8 \cdot 10^{-4}$, close to the numerically ideal $F_{1, \text { opt }}=1 / 2048$. In practise, of course, a lower magnification $(\leq 150)$ is adjusted with error margins for manual alignment as detailed later, matching the optimum Fresnel number even better.

The glass capillary absorbs between $23 \%$ and $42 \%$ of the radiation (values from [32] for $\mathrm{SiO}_{2}$ with a thickness of $50 \mu \mathrm{m}$ and $100 \mu \mathrm{m}$, respectively), depending on its orientation. This loss is acceptable for illumination with a $1 \mathrm{~d}$ WG. We evaluated the practical minimum exposure time to $5 \mathrm{~ms}$, allowing for a repetition rate of $7 \mathrm{~ms}$ corresponding to a maximum of $142 \mathrm{FPS}$. With the maximum rotation speed of $1 \mathrm{~Hz}$, a feature in the corner of the capillary at a radius of $35 \mu \mathrm{m}$ has been moved about $1.1 \mu \mathrm{m}$ during this exposure time. If the capillary is not adjusted precisely in the centre of rotation, the velocity is even higher. To guarantee that such a feature can still be recognised as such in a single projection, we used $\mathrm{SiO}_{2}$ spheres with a diameter of $(2 \pm 0.2) \mu \mathrm{m}$, approximately twice the maximum blur, dispersed in water with a solid content of initially $5 \%$ (Fluka Analytical). The maximum absorption of radiation of such a bead is less than $0.7 \%$ with respect to the surrounding water while the relative phase shift is up to $0.3 \mathrm{rad}$. Thus we can expect a distinct signal of each marker, exceeding the limits of a weakly interacting object for higher concentrations. But even in that case the net relative phase shift in a projection is to be expected less than $2 \pi$, in principle allowing for the usage of iterative phase retrieval without the problem of phase-wrapping.

The particle motion is mainly driven by the gravitational force $F_{g}=\Delta \varrho V_{s p h} . g$ with the density difference $\Delta \varrho=\varrho_{\mathrm{SiO}_{2}}-\varrho_{\mathrm{H}_{2} \mathrm{O}}$, the sphere volume $V_{s p h}$. and the gravitational acceleration $g$, exceeding the highest centrifugal force $F_{z}=\Delta \varrho V_{s p h} r \omega^{2}$ occurring in the capillary at a rotation speed of $\omega=2 \pi / \mathrm{s}$ by a factor of more than $4 \cdot 10^{4}$, allowing to neglect rotation-induced effects. In addition, the water column can be expected to follow the rotation without a noticeable delay. The sedimentation speed of a single particle

$$
v=\frac{2}{9} \frac{\Delta \varrho a^{2} g}{\eta}
$$


can be approximated by the already mentioned STOKES' law, equalising $F_{g}$ with the frictional force $F_{d}=6 \pi \eta a v$ acting on a sphere with radius $a$ in a fluid with viscosity $\eta$ at small REYNOLDS numbers $\varrho v a / \eta \ll 1$. For $2 \mu$ m diameter $\mathrm{SiO}_{2}$ beads $\left(\varrho_{\mathrm{SiO}_{2}} \approx\right.$ $\left.2.4 \mathrm{~g} / \mathrm{cm}^{3}\right)$ in water $(\eta=1 \mathrm{mPa} \cdot \mathrm{s})$ at $T=293 \mathrm{~K}$ room temperature, we expect

$$
v_{S} \approx 3 \mu \mathrm{m} / \mathrm{s},
$$

corresponding to a negligible motion of $15 \mathrm{~nm}$ during the minimum exposure time. Depending on the bead concentration, the water displaced by the sinking spheres streams up and further reduces the mean sedimentation velocity. This motion is superposed by random Brownian motion. For the present case, the diffusion constant

$$
D=\frac{k_{B} T}{6 \pi \eta a}
$$

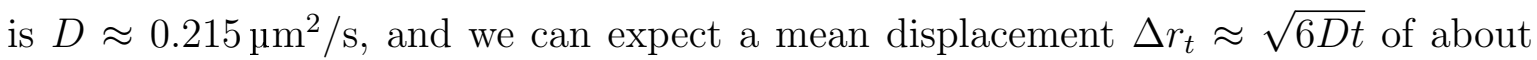
$\Delta r_{1 \mathrm{~s}} \approx 1.13 \mu \mathrm{m}$ per second and $\Delta r_{5 \mathrm{~ms}} \approx 80 \mathrm{~nm}$ during the minimum exposure time. This meets the requirements of a larger velocity along the rotation axis then perpendicular to it, as well as clear but not too fast motion within the acquisition time of a sinogram. With this, the Péclet number

$$
P e=a \frac{v_{S}}{D} \approx 14
$$

is in the challenging regime where neither advective nor diffusive motions can be neglected.

\subsubsection{Sample preparation and measurement}

The samples were prepared on-site, about an hour before each experiment. The capillaries were cut to pieces with a length between $15 \mathrm{~mm}$ and $25 \mathrm{~mm}$ by a scalpel, leaving a clean cut with only few cracks in the glass wall. One end was dipped in the suspension of $\mathrm{SiO}_{2}$ particles in water, properly dispersed by a vortex mixer, which filled the compartment completely due to capillary forces. Both ends were immediately closed by sticking them into Haematocrit sealing compound (BRAND GmbH \& Co. KG) creating a plug of $1 \mathrm{~mm}$ to $3 \mathrm{~mm}$ in the capillary. Importantly, the formation of air bubbles at both ends had to be avoided, otherwise a heavily moving water-column bouncing on the lower air buffer would have spoiled the measurement. Since the air stayed fixed at the ends, a small bubble on the upper side was acceptable. Subsequently, both ends were sealed air-tight with nail polish to prevent evaporation. The Haematocrit served as a buffer preventing immediate contact of the water content and the varnish, otherwise impeding the sealing. So-prepared capillaries were stored upright, with a possible air-bubble (the later top side) on the low end accumulating the particles there. The state of sedimentation could be controlled easily by the transparency of the capillary in a microscope (or even directly by eye). Transparent capillaries with the large majority of spheres in the lower (later upper) region of the sample were used for the experiment. 
However, sample could not be used after too long a period because the spheres would clump otherwise.

For general alignment of the setup rotation axis, we used a capillary with larger diameter and dirt on the outside. Perfectly aligned, such a feature moves only horizontally in the projections during a full rotation. A non-horizontal line (tilt between detector and rotation axis) can be corrected afterwards by numerically rotating the detector images. In contrast, features moving on an oval-shaped path indicate misalignment of the (yaw) angle between optical axis and rotation axis, which is difficult to correct afterwards. Thus, the rotation stage had been adjusted to not see such an oval path but a horizontal line before the dynamic measurements. To mount the samples in the setup, a sample holder was filled with Haematocrit and the capillary was stuck inside as straight as possible with the particle-poor side - up to the level of sealing compound inside the capillary. Then, the sample was arranged on the rotation stage. Since the colloids started to sediment to the downward side at this point, the next steps of tomographic adjustment were carried out as fast as possible.

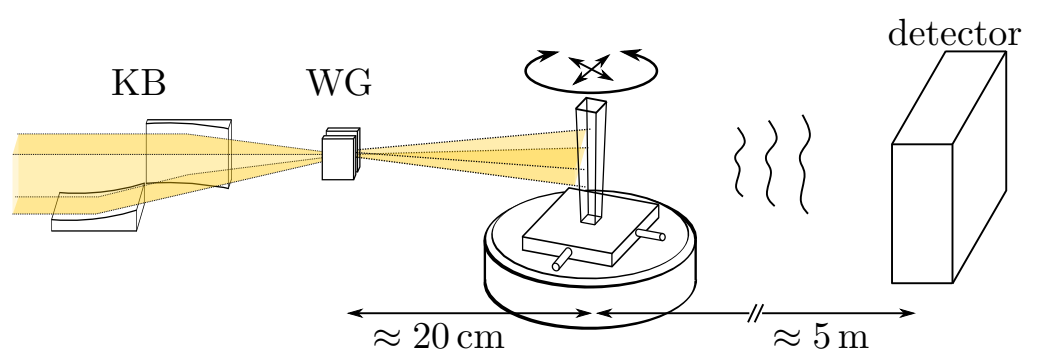

Figure 6.8: GINIX setup for fast tomography. At a distance of about $20 \mathrm{~cm}$ behind a $1 \mathrm{~d}$ WG the sample is mounted on a manually adjustable $x-y$-stage, sitting on a rotation motor which can be positioned remotely.

For manual alignment of the sample on the rotation axis and of both of them in the centre of the X-ray beam, the OAV microscope was used where the true beam position had been marked before. First, the capillary was moved into the field-of view of the microscope with the micrometre screws above the rotation. Then, the stage was rotated until the capillary reached the leftmost position in the projection. During this process (when the capillary left the microscope's FOV) the entire stage was moved by the $x$ motor to keep the sample in the centre of the microscope. After reaching the leftmost position, the stage was rotated about $180^{\circ}$ and the capillary, now at the rightmost edge, was again adjusted in the centre of the microscope by moving the $x$-motor. The rotation axis is in the centre of this $x$-distance. Thus, the $x$-motor was moved to the rotation axis and the capillary readjusted to the center of the microscope by the micrometre screws. For the orthogonal direction, the stage was rotated about $90^{\circ}$ and the capillary again adjusted by the micrometre screws. Since usually both screws have to be moved for this adjustment, this step had to be iterated several times for the orthogonal positions until the capillary showed no lateral movement during rotation. This alignment was carried out within a few minutes with remarkable accuracy. After the hutch was sealed and the X-rays shutter opened, the WG was re-aligned by a macro optimising the total intensity, and the camera ROI and binning were adjusted. An interesting region in the capillary, e.g. the upper edge of the already 
sedimented spheres, was selected by moving the $z$-motor beneath the rotation stage. When doing so, even a slightly tilted capillary in the Haematocrit-filled holder moves out of the centre of rotation. Thus we allowed some error margin in the detector FOV by relaxing the magnification to a value around 100. For fast tomography, the camera was started in series mode with pre-set exposure time and framerate and the rotation was started from $-3600^{\circ}$ to $3600^{\circ}$ ( 40 rotations), where acceleration and deceleration took about one full rotation each. After the rotation, the sample was moved out of the beam to capture several hundred flat-field-images before the acquisition was stopped manually. Series with different combinations of frame rate, exposure time and rotation speed (including zero rotation) at promising regions of the sample were recorded.

\subsection{Data analysis}

This chapter follows the data analysis of a sample measured with the shortest exposure time of $5 \mathrm{~ms}$ and a repetition interval of $7 \mathrm{~ms}$ (142.86 FPS). With $2 \times 2$ binning (resulting pixel size of $13 \mu \mathrm{m})$, a ROI of $1024 \mathrm{px} \times 512 \mathrm{px}(x \times y)$ had been chosen, illuminated by a $1 \mathrm{~d}$ WG narrow along the $x$-direction. 6975 images were recorded, including 5700 projections with constant maximum rotation speed covering a process time of $39.9 \mathrm{~s}$. The sample shows sedimenting particles in the upper two thirds adding to a slowly rising region of already sedimented spheres in the lower third. The Fresnel number $F_{1} \approx 0.002$ clearly indicates the holographic regime. With a magnification of about 112 , the effective pixel size is $116 \mathrm{~nm}$.

\subsubsection{Data conditioning and alignment}

A typical projection is shown in Fig. 6.9 next to the flat-field shaped by the 1d WG. The result of the flat-field-correction is depicted in (c). Alternatively, each hologram can be divided by the mean of all holograms, since the moving object structure is averaged out over all rotations (see (d)). With the drawback of losing quantitation, this step removes all static features in the images. Here, both corrections were carried out, i.e. the 'mean-field correction' was based on already flat-field corrected data. A typical result is shown in Fig. 6.9 (e).

Fig. 6.10 shows the sinogram obtained from a central cut through the stack of all $N$ images. The fluctuating intensities can be attributed to the WG vibrating in the $\mathrm{KB}$ focus which does not impair the structure of the illumination importantly but influences the brightness. The orientation of the capillary can be easily estimated. Its square shape leads to four "bumps" in the sinogram with every full turn: At each bump, it is oriented diagonal with respect to the illumination, whereas at each "kink", it is oriented perpendicular to the beam. Since the capillary was not exactly in the centre of rotation, the whole projections additionally show a lateral displacement according to a sine wave for each four bumps, which has been used to determine the rotation velocity. To this end, the $j=1 \ldots N$ rows of the sinogram are shifted according to the function $f(\alpha)=A \sin \left(\alpha_{0}+j \pi / N_{\alpha}\right)$ until amplitude $A$, phase-offset $\alpha_{0}$ and the number 
(a) flat-field
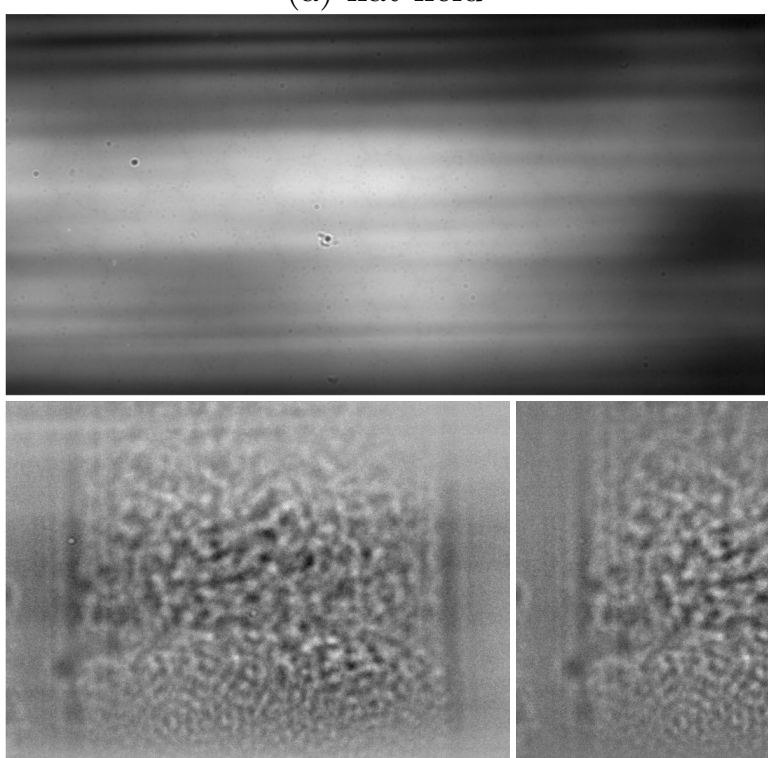

(c) flat-field correction

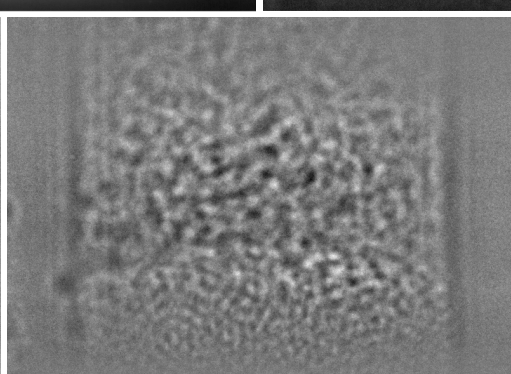

(d) mean-field correction (b) typical detector image
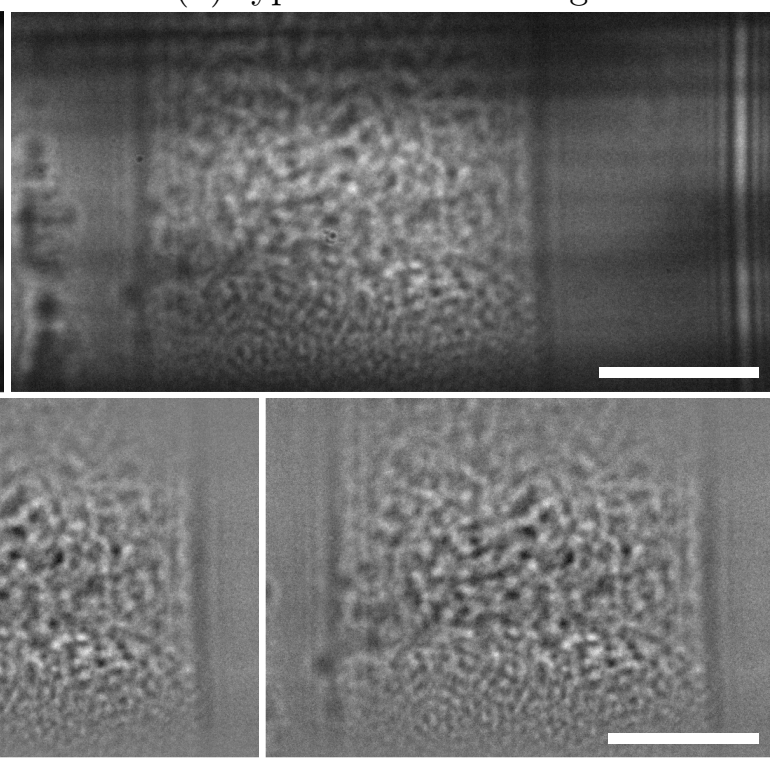

(e) both corrections

Figure 6.9: Flat and mean-field correction of the raw detector data. While the flat-field correction depicted in (c) ((b) divided point-wise by (a)) still contains some artefacts from the illumination, see e.g. the top left region, the mean-field correction in (d) ((b) divided point-wise by the mean of all detector images of the moving sample) removes those artefacts. The mean-field correction of previously flat-field corrected data as depicted in (e) has been used in the further analysis. The grey values (white is brightest) are in arbitrary units, the scale bars valid for the corresponding row indicate a width of $25 \mu \mathrm{m}$.

of angles per sinogram $N_{\alpha}$ have been optimised such that the sinogram is aligned symmetrically as depicted in Fig. 6.10. The correction shows that the rotation was very stable during the measurement. Neither lateral drift nor an increasing misalignment is visible, beginning and end of the sinogram are equally well aligned. Here, we found $N_{\alpha}=80.35$ angles per sinogram, corresponding to a rotation velocity of $320^{\circ} / \mathrm{s}$. The measured $N=5700$ projections thus cover 39.9 s of the sedimentation process in about 35.5 rotations. Note that the corrections shown in the figure do not have to be carried out in this step, saving an unnecessary interpolation. Only the value of $N_{\alpha}$ is of importance.

A second important value to determine is the position of the rotation axis in the images. For this purpose, a hologram was compared with a hologram recorded from the opposite side of the sample, flipped horizontally. In a perfectly aligned experiment, the static parts of the sample, i.e. the capillary, appear identically in both pictures and thus cancel out in the difference image. In practise, the relative shift and rotation of both images is optimised to remove the capillary as well as possible in the difference image. All images were corrected for the rotation only, noting the shift for later correction.

Fig. 6.11 depicts the so-called linogram obtained by integrating the stack of all holograms along the $x$-direction, smoothened along the time axis for better visibility. It can be used to investigate axial drifts and vibrations of the sample. For a static object, the integrated intensity profile is constant over all projections and only horizontal 


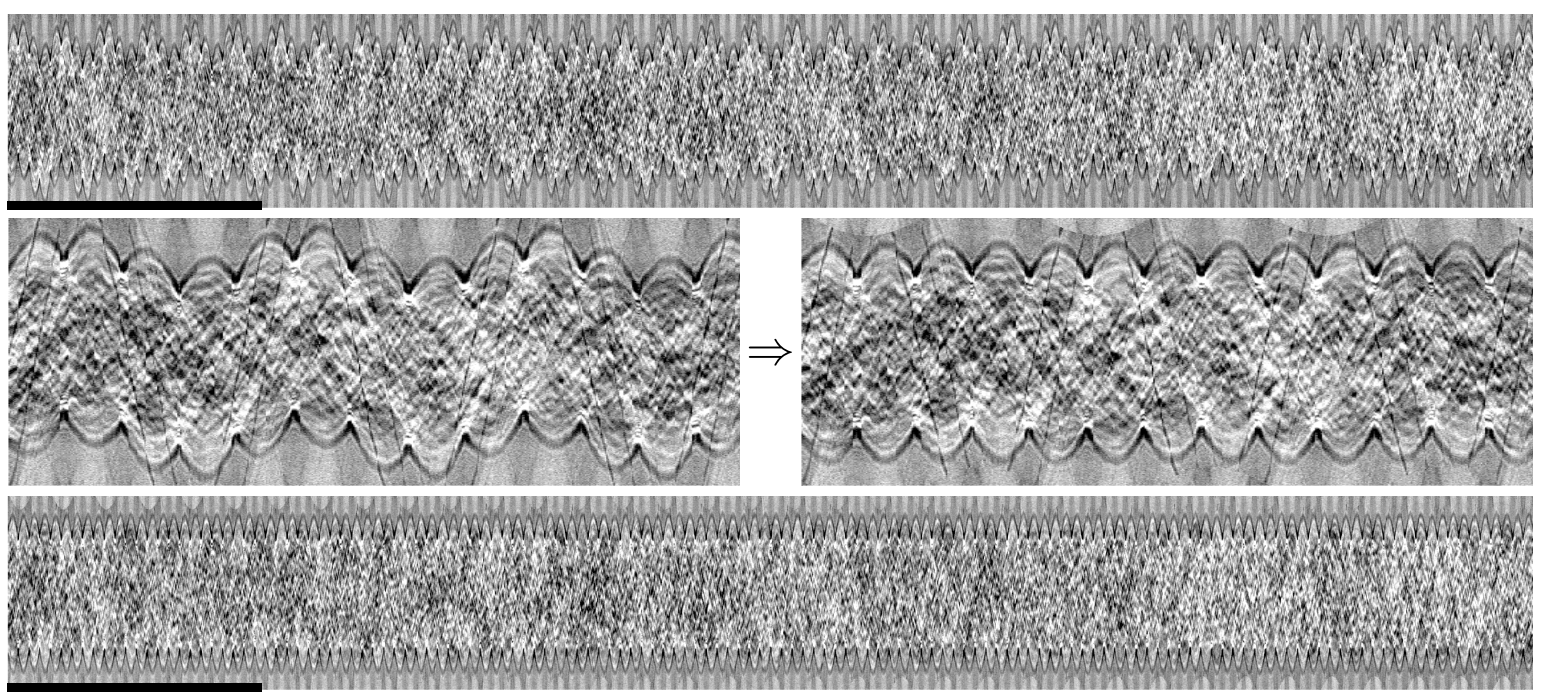

Figure 6.10: The central slice trough the stack of all holograms reveals the sinogram on top. The orientation of the quadratic cross section capillary can be clearly seen. The detail indicated by the black bar is enlarged in the center left. With the alignment explained in the text, the rotation axis is corrected, resulting in the sinogram at the bottom and detailed in the center right.

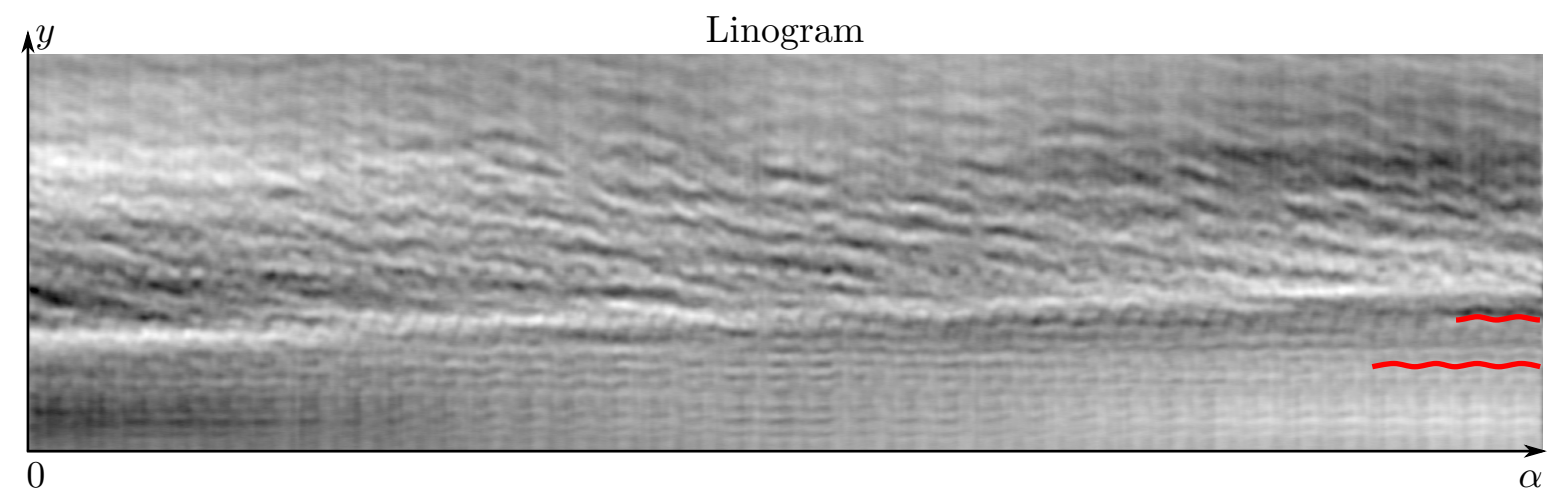

Figure 6.11: In the so-called linogram, obtained by integrating the stack of holograms along the $x$ direction, a wobble with the same period as the rotation can be seen (red line).

lines appear in the linogram. However, in case of dynamic tomography we get a first preview of the dynamics: one can clearly see the lower region of sedimented particles slowly rising while matter is sinking down as indicated by the diagonal patterns in the vertical centre region of the linogram. Nevertheless, the constant region at the bottom also reveals small oscillations with the same period as the rotation, possibly originating from an interim misalignment of the rotation axis around the $x$-axis (yaw angle), a wobble of the rotation stage, or most likely a vibration of the whole sample tower induced by the rapidly rotating and not perfectly balanced mass on top. This wobble was determined analogue to the above case of the sinogram. Additionally, all holograms were cross-correlated to their 3 precursors and 3 successors to reveal high 
frequency vibrations. All of the obtained shifts, i.e. both rotation axis offsets, $y$-axis wobble, and cross-correlation shift were then corrected with a single interpolation.

At this point, many methods of phase-retrieval and tomographic reconstruction are possible. The holograms show a very diverse composition, with a strong signal from the capillary walls dominating the weak and sometimes blurry patterns originating from its content. However, it can be removed easily either before or after the reconstruction: By averaging many projections from the same direction blurring the dynamics, a projection of the static parts of the object can be obtained and subtracted from the corresponding projections, leaving only the dynamic parts. Thus, we can treat the remaining object as being weak in good approximation. This allows for a conventional two step reconstruction with phase retrieval based on the CTF and tomographic reconstruction relying on the FBP. Several different schemes were tested, including a normalised cross-correlation based position estimation of the beads directly from the holograms and an iterative 3d-propagation based approach. However, since the dynamics of the markers, rather than the quantitative sample structure, are if interest a considerable benefit of those attempts could not be found. In the opposite, the conventional two-step method performs remarkably well with the sliding-window approach detailed in the following sections.
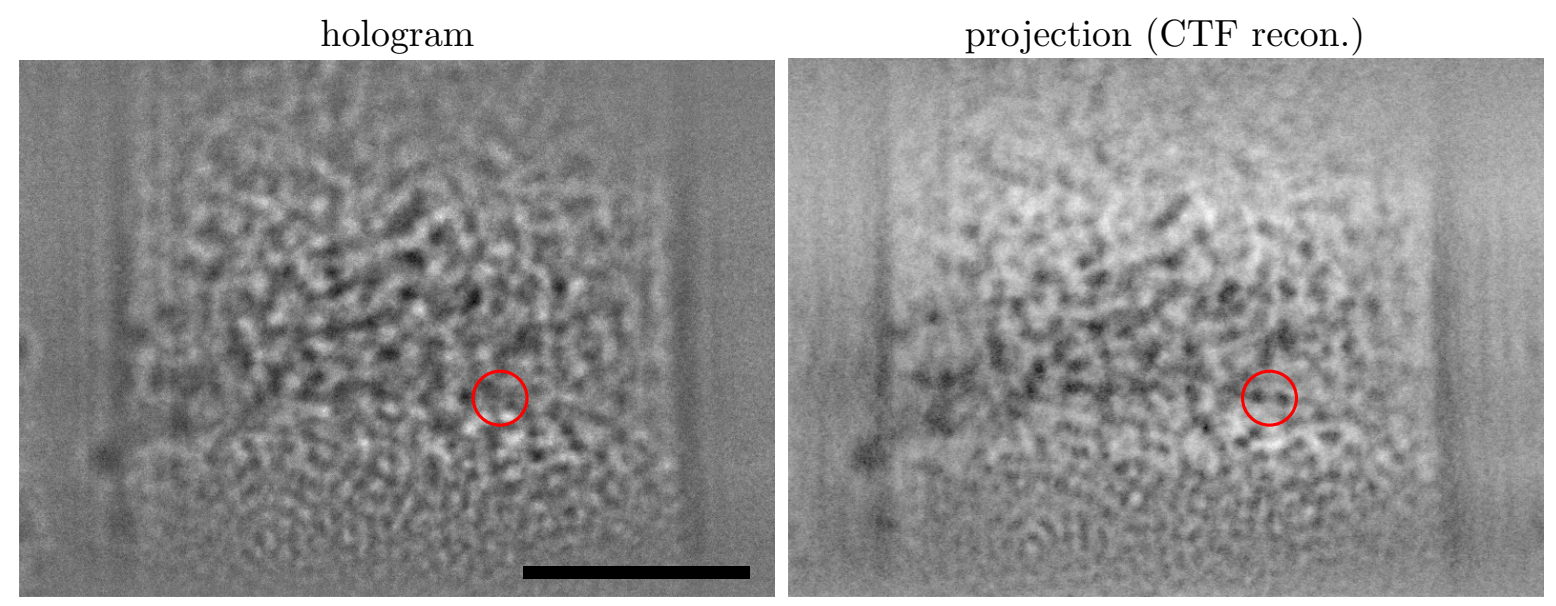

Figure 6.12: CTF-based phase retrieval of a typical hologram. Note that in the reconstruction, markers emerge which are not visible in the hologram, as indicated by the red circles. The scale bar is $25 \mu \mathrm{m}$. See text for details about the CTF parameters.

A typical CTF result is shown in Fig. 6.12 for an effective Fresnel number of $F_{1}=$ $2.1 \cdot 10^{-3}$ as well as the parameters $a_{2}=0.02, \sigma_{c u t}=0.001$ and $\beta / \delta=0.1$, yielding better results than the theoretical ratio of $\beta / \delta \approx 0.023$ of the relative absorption and phase shift with respect to the surrounding water (see Eq. (5.27) for the definition of the parameters). Since the quality of the projection does not seem to be improved at all on the first look, it is important to note that some markers emerge at positions, where no such signal is visible in the holograms, as indicated in the figure. Thus, signals that might look like markers in the hologram do not necessarily indicate the presence of a real glass bead. Omitting the step of phase retrieval would introduce many artefacts and inconsistencies during further processing. 

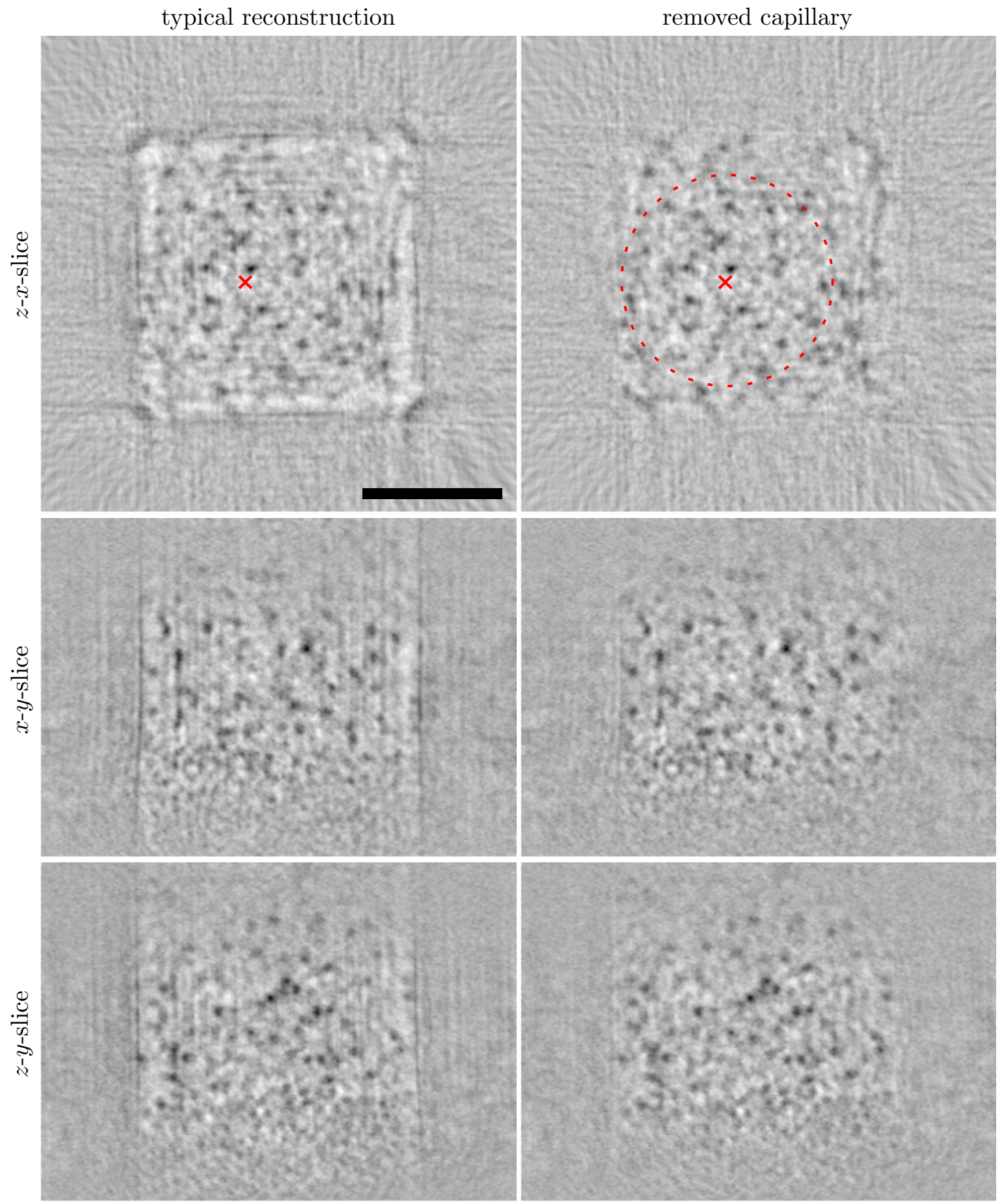

Figure 6.13: Orthogonal central slices through a typical FBP reconstruction of the capillary (left). The original axis of rotation is highlighted by a red cross. Clearly, the signal gets worse at larger distances from this point. On the right side, the mean of all time frames was subtracted from the volume, removing static features such as the capillary and associated artefacts but also the resting spheres in the lower region of the capillary. 
For tomographic reconstruction, the data was binned $2 \times 2$ and a sliding window approach was used, i.e. the $N_{\alpha}$-sized window of projections used for the reconstruction was shifted in steps of $N_{\alpha} / 4$ over the entire sinogram, yielding a total of 283 reconstructed volumes with overlapping process time intervals, denoted as time frames in the following. The central slices orthogonal to the $x, y, z$ axis of such a typical reconstruction are shown in Fig. 6.13. To remove the static portions, all time frames were averaged and subtracted from each single volume. This safely removes the capillary signal clearly improving the quality but also the already sedimented spheres are removed, as can be seen in the figure. In the $x-z$ 'top' view, orthogonal to the axis of rotation, the features at the capillary corners are clearly blurred compared to those in the centre. This effect can be observed in all time frames and can, for the most part, be attributed to the rotational blurring of markers distant from the centre of rotation. To this end, the original axis of rotation has been highlighted, located in the centre of the sharpest region. Since the blur affects each detector image, it cannot be compensated in the direct $3 \mathrm{~d}$ reconstruction, imposing a fundamental limit for the proportion of angular velocity $\omega$, marker size $a$, sample diameter $r$, and exposure time:

$$
\omega r<\frac{2 a}{\Delta_{t}}
$$

A particle at a distance $r$ to the axis of rotation can only be reconstructed as such, if it is displaced about less than its diameter $2 a$ in the exposure time interval $\Delta_{t}$.

\subsubsection{3d particle tracking}

To follow the path of the visible micro-spheres, a two-step particle tracking procedure was carried out consisting of first locating the particles in each volume individually followed by a nearest-neighbour connection in time for tracking. For proper localisation, all volumes were convolved with a model of an ideal sphere, smoothing the signal to remove outliers due to noise. Then, the voxel with the highest signal was selected as the most probable position of a marker and symmetrically replaced by a zero valued sphere with twice the bead radius, since in this volume no second centre of a bead can occur. This maximum-searching and replacement procedure was repeated until a signal threshold was reached, resulting in a mean of about 4230 located candidates in each volume. The threshold was chosen by visual inspection such that the majority of spheres were correctly identified with only a small number of falsely recognized features. A typical result of the procedure is shown in Fig. 6.14, with highlighted regions where motion artefacts lead to false matches. Such false positive positions are removed in the next step of tracking the particles. For this purpose, each position was connected to its nearest neighbour in the succeeding volume, provided the distance was smaller than a bead radius of $4 \mathrm{px}$. Gaps in the paths were closed by connecting ending and beginning frames with a distance of less than 6 px over a gap of one time frame. The minimum accepted path length was 5 time steps, i.e. a bead present over the range of more than a full sinogram. A number of 51705 paths were found by this procedure, as illustrated in Fig. 6.15, the longest amongst them reaching over 192 time frames, with 156 paths having a length of at least 100 time frames and about 1700 paths with 


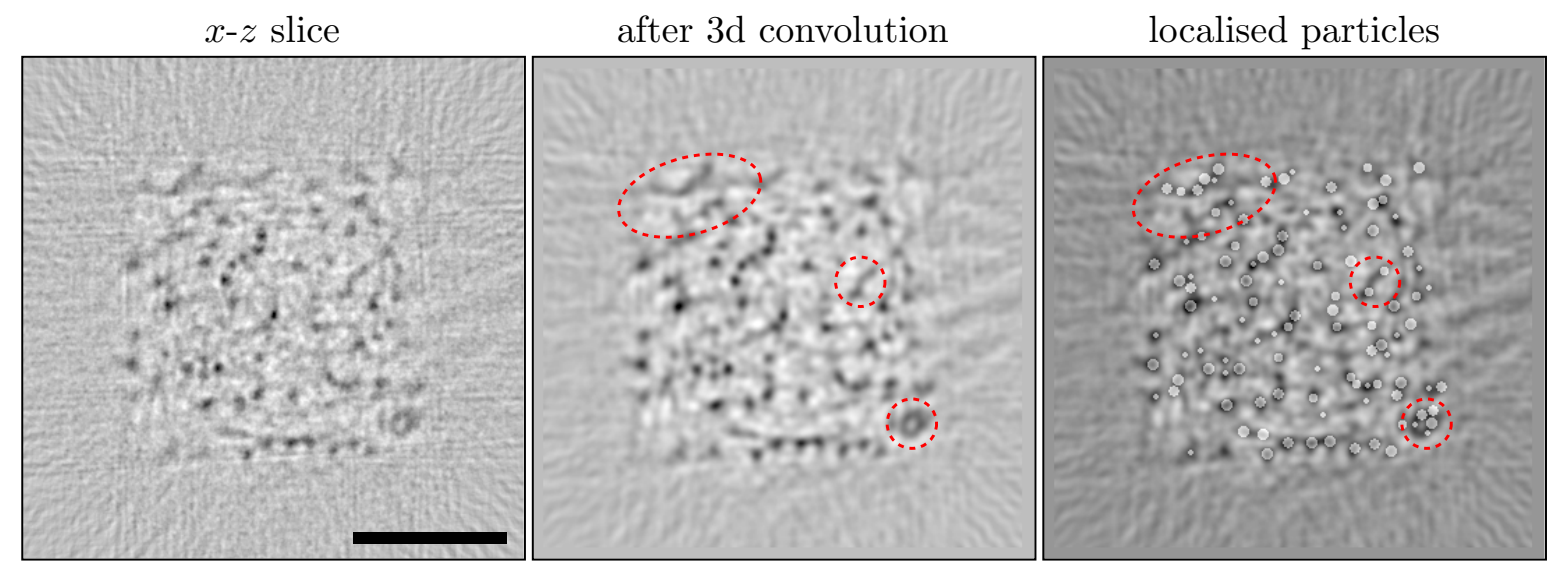

Figure 6.14: Localisation procedure of particles in a typical $x$ - $z$ slice of a time-frame (left). The volume is convolved in $3 \mathrm{~d}$ with a model of an ideal sphere, resulting in the slice depicted in the centre image. The maxima (black dots) are identified as the most probable positions of the spheres, as highlighted in the right image. Note that a nonhighlighted dot does not necessarily indicate a missing sphere, since the signal might be elongated along the $y$ direction and marked in other slices. Artefacts due to blurring of fast markers are highlighted by red lines. They lead to false positive positions which are removed in the next step of tracking. The scale bar is $25 \mu \mathrm{m}$.

a length of at least 50 time frames. Clearly, many of the paths are broken parts of the same marker's trace, but still many individual cases can be studied in great detail. The $N=1,10,10^{2}, 10^{3}, 10^{4}$ longest paths are visualised in Fig. 6.16.

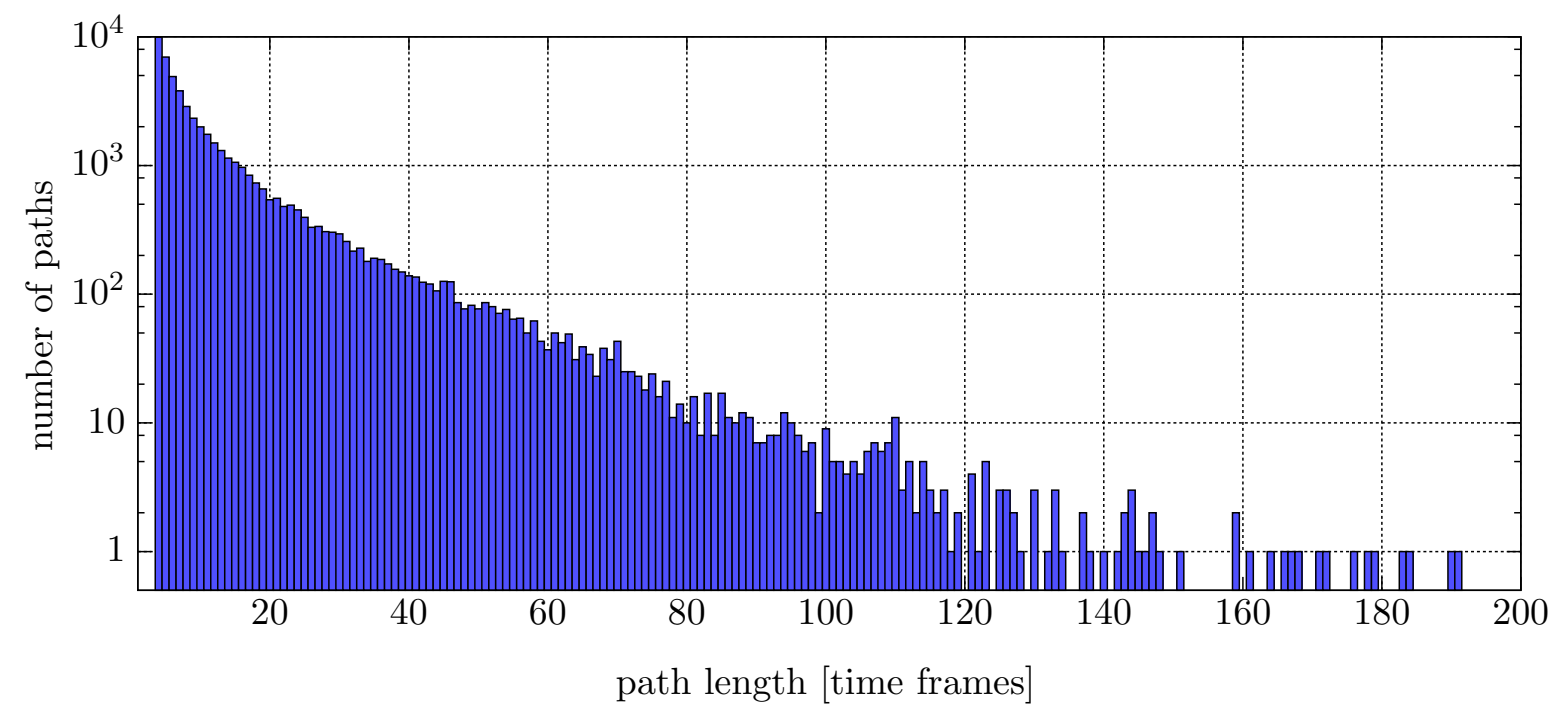

Figure 6.15: Histogram of reconstructed path length with a minimum observed length of 5 and a maximum of 192 time steps.

Many aspects of the particle behaviour can already be extracted from the longest trace $N=1$. Initially, the sphere sediments predominantly vertically (and with a high 
velocity, not shown) towards the bottom of the capillary. Then, the behaviour changes drastically: the particle exhibits a much more pronounced horizontal movement, while the vertical motion is stopped - the sphere even shows a slow ascension. This happens in the middle of the volume, well above the already settled particles. Such a strong sideways motion can also be observed for the majority of the other particles at $N=10$ and $N=100$. An undisturbed diffusion in the plane perpendicular to the direction of sedimentation would result in compact, "curly" paths in the $x-z$ view of Fig. 6.16. Especially at $N=100$, only very few spheres display this type of behaviour, while most paths are clearly elongated. However, no preferred direction of this elongation is immediately obvious.

For $N=1000$ particles, the top view reveals a significantly higher concentration of the longest (i.e. the best detected) paths in the bottom left area. When comparing with Fig. 6.13, it becomes obvious that this is the periphery of the true rotation axis. In this region, single spheres are affected less by rotational blurring within the exposure time of single images, resulting in a better recognition of the spheres and thus the paths. For $N=10000$, no single traces can be distinguished anymore. However, the side views show a horizontal area with smaller particle density at the upper edge of the field of view. Here, the illumination had a very low intensity. As a result, the contents of this area could not be reconstructed, despite flat- and mean-field correction - the contrast was simply too weak. 

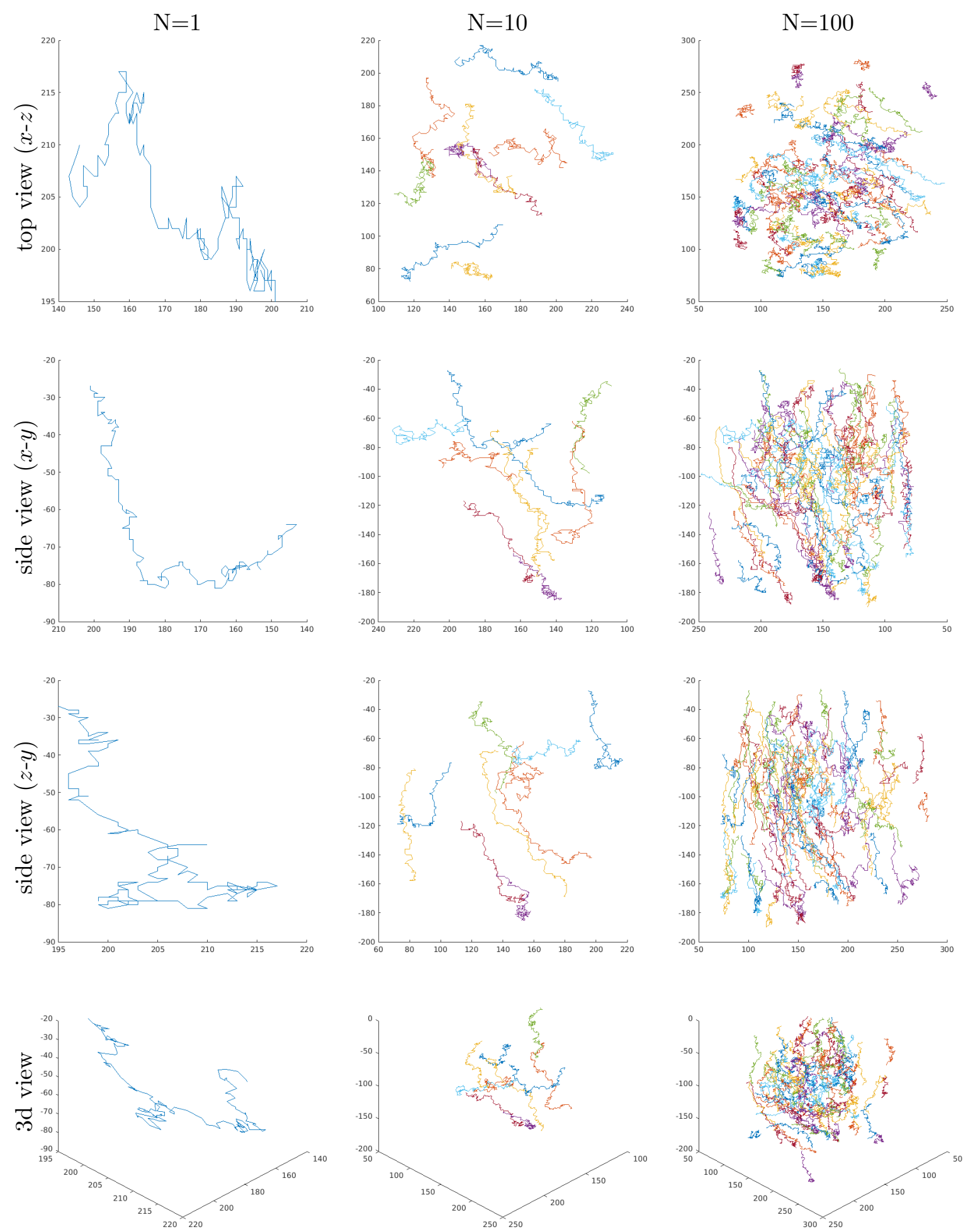

Figure 6.16: $N=1,10,100$ longest trajectories showing sedimentation but also occasional upflow and elongated paths in the $x-z$ directions. All values are in pixel, corresponding to about $232 \mathrm{~nm}$. 

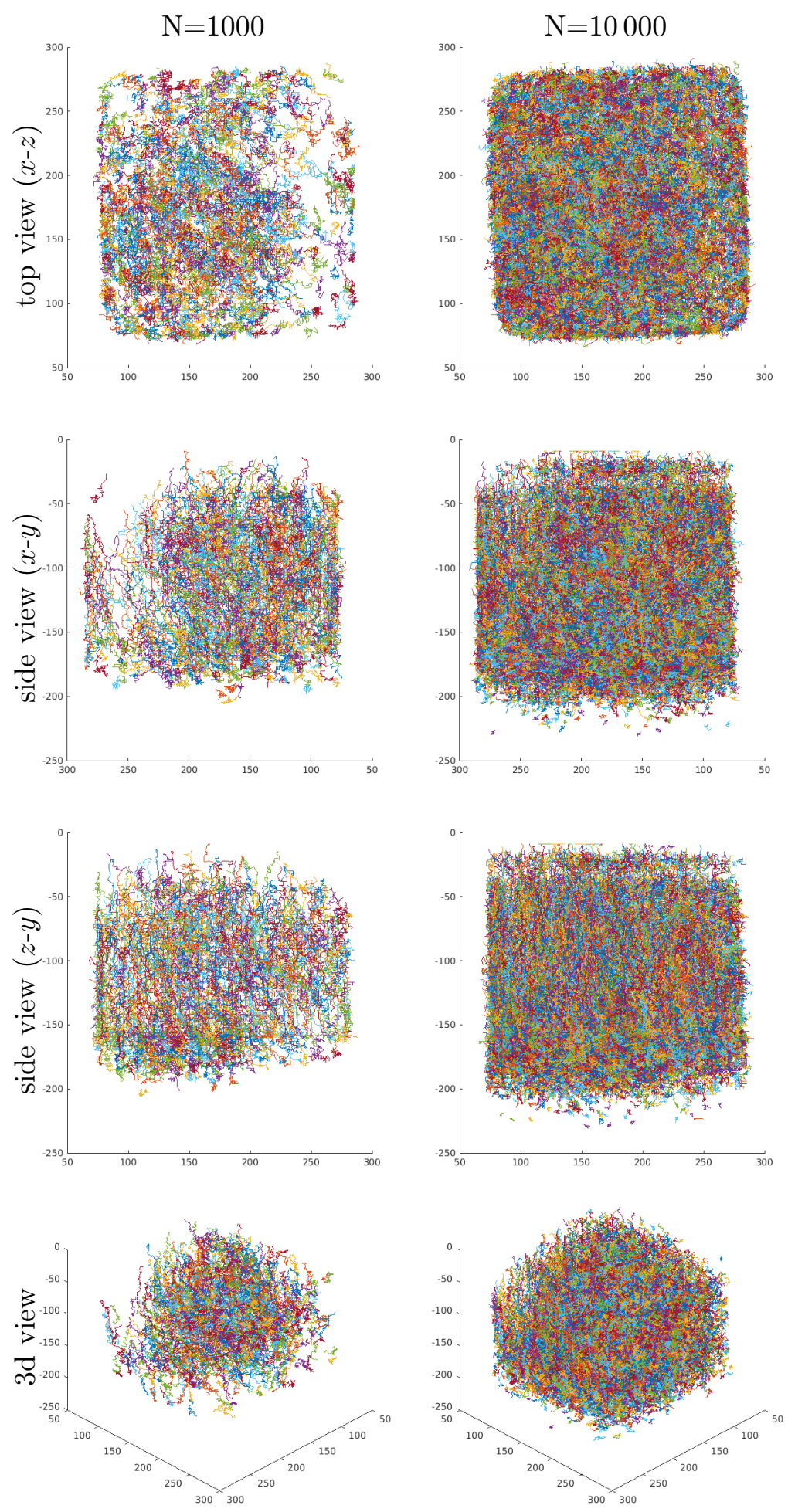

Figure 6.17: $N=10^{3}, 10^{4}$ longest trajectories. For $N=10^{3}$ clearly a region can be identified where the density of reconstructed trajectories is particularly high. For $N=10^{4}$ the entire volume is covered by trajectories revealing a horizontal low-density region at the upper side of the volume (in $x-y$ and $z-y$ views) where the illumination was particularly dark, resulting in insufficient contrast. All values are in pixel, corresponding to about $232 \mathrm{~nm}$. 


\subsubsection{Collective behaviour}

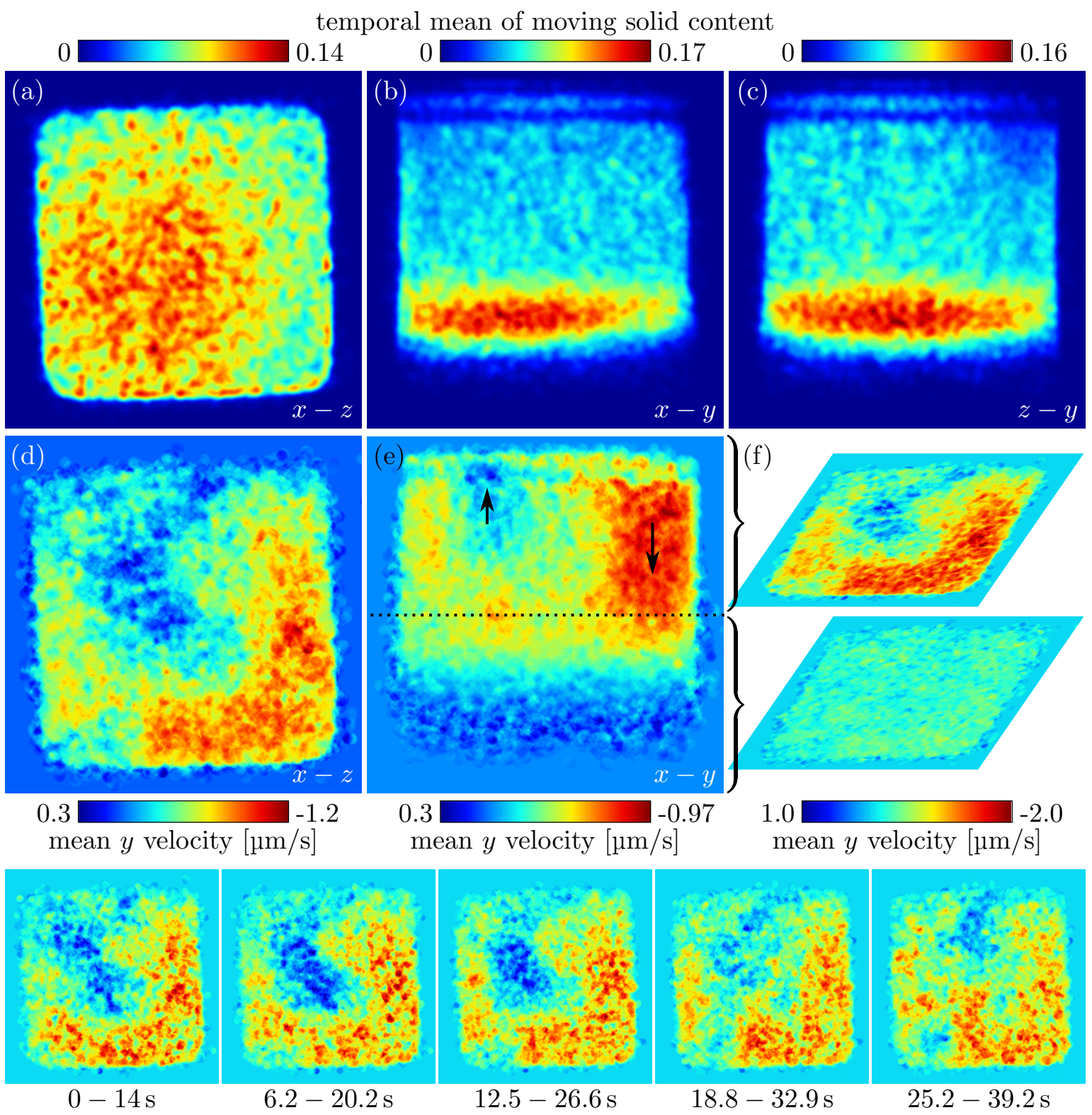

Figure 6.18: Temporal mean density of moving particles and collective dynamics in the capillary. The top row depicts a top (a) and two side views (b),(c) of the mean density, while the central row shows a top (d) and a side view (e) of the mean vertical particle velocity per voxel. See the text for a more detailed description. Regions of sinking spheres (red) can be clearly distinguished from regions of rising spheres (blue). Since these collective dynamics are more pronounced in the top half of the volume, the bottom row depicts the temporal evolution of the currents only for this region, time-averaged in the specified intervals. The colour bar of (f) applies for the bottom row, too.

To better investigate the complex particle behaviour during the sedimentation process with large lateral displacements and even occasionally rising beads, a total of 23000 paths with a minimum length of 10 time frames were analysed as depicted in Fig. 
6.18. First, the temporal mean particle density was calculated by averaging all detected positions of spheres, represented by ideal spheres, of the 23000 paths over all time frames. By averaging this volume along one of the coordinate axes, we obtain the temporal mean fraction of solid content in the corresponding direction as shown in the top row of Fig. 6.18. Panel (a) displays the already mentioned maximum in the area surrounding the true axis of rotation in the bottom left corner, while panels (b) and (c) clearly show a high particle density in the lower part of the capillary, where the sedimenting particles come to rest. Insight in the collective dynamics can be obtained by averaging the sedimentation speed as illustrated in Fig. 6.18 (d)-(f). For this figure, each detected position of the 23000 longest trajectories was represented by the ideal sphere weighted with the corresponding velocity in $z$-direction. The sum of all those spheres along one of the coordinate axes normalised by the corresponding sum of the temporal mean particle density reveals the mean velocity per voxel in the voxel columns. Clearly, a region of high sinking velocity (red) can be distinguished from a region of slowly rising particles (blue). The sinking spheres displace water which wells up and impedes the sedimentation of spheres in this area, or can even carry them upwards, albeit with smaller climbing rate than sedimentation velocity. In the lower half of the capillary, the velocities are small, the magnitude of the flow increases towards the top, as can be seen in the side view (e). This is illustrated further in panel (f), which displays $z$-averages of the top and bottom half of the 3 d velocity distribution. Finally, the bottom row of Fig. 6.18 displays the temporal evolution of these currents in the top half of the capillary (calculated as explained above, but only taking into account the spheres that were detected in the relevant time interval). The images show that a complex, slowly varying flow evolved during the measurement.
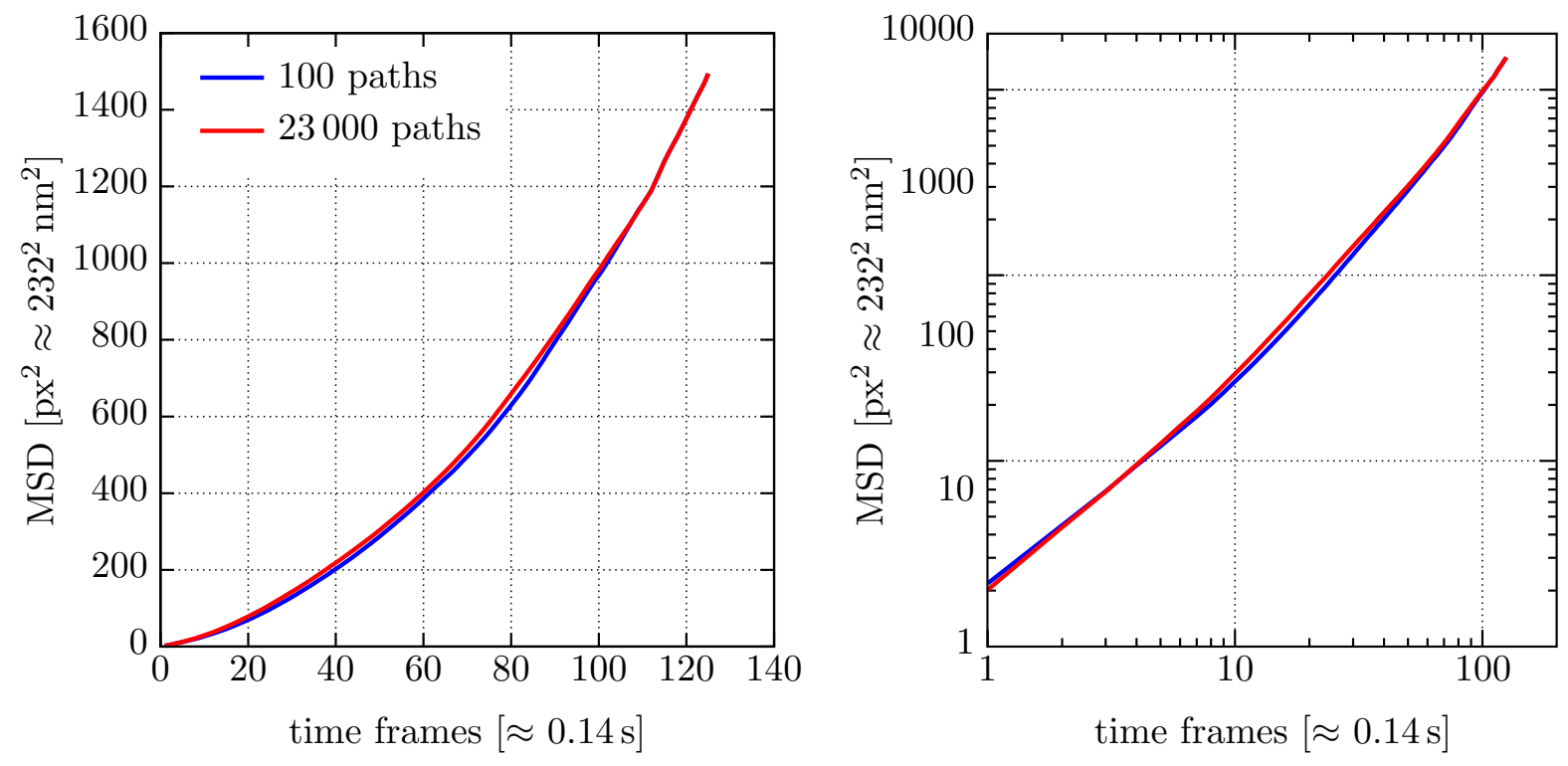

Figure 6.19: Mean squared displacement of sedimenting spheres, perpendicular to the direction of gravity, obtained by including only the 100 longest trajectories and all 23000 paths with a minimum length of 10 time steps. The increase clearly indicates superdiffusion. 
Similar currents can be observed along the other directions, albeit at much slower speeds. The mean particle velocity along the $x$-direction is $\bar{v}_{x} \approx-102 \mathrm{~nm} / \mathrm{s}$ with a standard deviation of $\sigma_{v_{x}} \approx 788 \mathrm{~nm} / \mathrm{s}$, while the mean velocity along the $z$-direction is $\bar{v}_{z} \approx 112 \mathrm{~nm} / \mathrm{s}$ with $\sigma_{v_{z}} \approx 787 \mathrm{~nm} / \mathrm{s}$. For comparison, the mean particle velocity along the sedimentation direction is $\overline{v_{y}} \approx 560 \mathrm{~nm} / \mathrm{s}$ with $\sigma_{v_{y}} \approx 860 \mathrm{~nm} / \mathrm{s}$. Minimum and maximum flow in all directions match the maximum accepted velocity of $\pm 4 \mathrm{vx} /$ time frame, about $\pm 6.63 \mu \mathrm{m} / \mathrm{s}$. Due to these flows, diffusional movements of particles perpendicular to the $y$ direction are superposed by extended drifts. The mean squared displacement in these directions $(x, z)$, as depicted in Fig. 6.19, clearly shows an increase and thus indicates a super-diffusive behaviour of the particles. Fig. 6.20 summarises the velocity fluctuations in all directions.

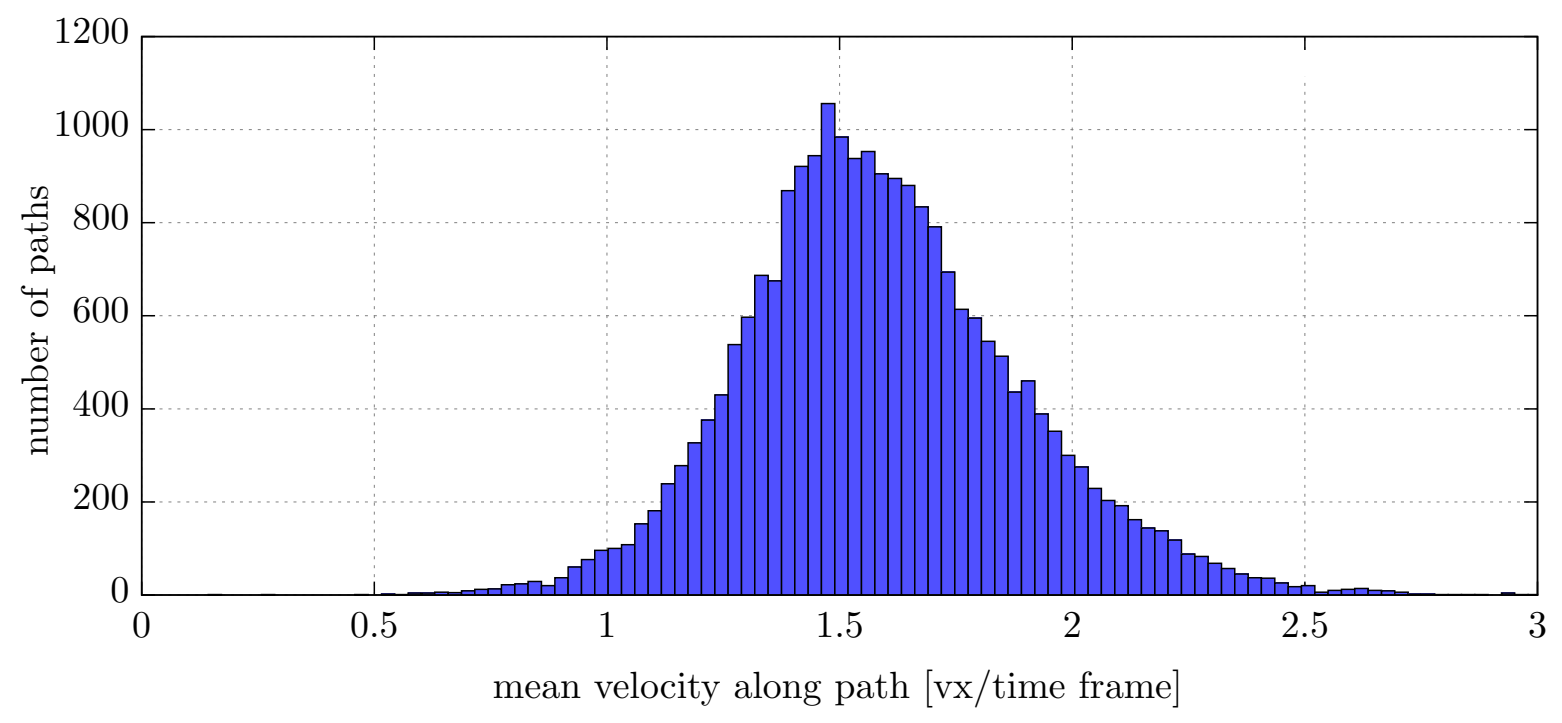

Figure 6.20: Histogram of the mean velocities of 23000 paths with a minimum length of 10 time frames. The unit of voxels per time frame corresponds to $1.66 \mu \mathrm{m} / \mathrm{s}$. 


\subsection{Summary and conclusion}

In this chapter, phase-contrast enabled CT was demonstrated revealing the dynamics of a complex colloidal system under realistic conditions in the challenging regime of a Péclet number of $P e=14$. Dynamics on the spatial order of less than the particle size as well as a temporal resolution less than the sinogram acquisition time $(<0.5 \mathrm{~s})$ have been reconstructed: The trajectories of thousands of single particles can be well observed and their collective behaviour reveals "invisible" self-induced complex water currents in the capillary

In this proof-of-principle demonstration, the system was very small compared to the size of the sedimenting spheres and the measurement took place at the interface to the sediment. Thus the process highly depends on wall effects usually avoided in order to reveal the pure process of settling. However, these boundary effects are of great relevance [94]. The dynamics at the lower interface seem to be particularly interesting. We had the impression that a large number of sinking particles lead to an amorphous deposition, while an almost crystalline arrangement is observed at low particle density. These phenomena have not been considered here and offer room for future evaluations. The experiment demonstrates the advantages and limits of time-resolved tomography for the determination of general motion. Notably, a major source of lost bead trajectories was motion blur during capillary rotation within the detector's exposure time, as can be seen in Fig. 6.13, 6.17 and 6.18. The best ways to compensate for this error would be a shorter exposure time, requiring a more intense illumination leading to higher radiation damage, or preferably a more efficient detection system. Since very fast particle motions suffer from the same blur and thus cannot be reconstructed properly or get excluded in the particle tracking, faster dynamics than observed here might occur in the sample. Indeed, the slower the motion, the higher is the probability of a complete detection over many time frames. Hence, the dynamics presented here have to be considered as a lower boundary of the motions occurring in the sample. However, since the lateral motion affected by positioning errors is still smaller than the rate of descent well observable even without rotation, faster dynamics can be considered as unlikely. The lateral motion with a mean in the sub-micrometre range during the acquisition time of a sinogram also allows neglecting positioning errors as described in the beginning of this chapter.

The results presented above clearly demonstrate that four-dimensional phase-contrast tomography can be used to investigate dynamics of weakly interacting specimen with high sensitivity - even though this high sensitivity enabled by X-ray waveguide illumination is highly challenging for fast tomography. 



\section{Dynamic tomography of a burning match}

The previous chapter adresses the $3 \mathrm{~d}$ motion of particles in a volume, tracked by a combination of phase retrieval and tomography. Localisation of the particles was possible with sufficient accuracy since their motion during the acquisition time of a sinogram was smaller than their diameter. However, inconsistencies can be seen to create many artefacts such as streaks that would make it difficult to elucidate the structure of an object, whereas they are still acceptable for the mere localisation of markers. As can be seen in the theoretical error estimations, the reconstruction improves significantly if motion gets incorporated into the reconstruction process, even in a first order approximation of constant velocity. However, in order to use this simple approach, the markers must already be recognisable individually in the projections. Since many samples lack well-trackable features of this kind, structure and motion have to be recovered in parallel.

In this chapter, an ansatz for determining motion and correcting for its artefacts in tomography is presented in the context of deforming matter. The concept is validated experimentally on data of a burning match, revealing the combustion process of the wooden structure in four dimensions. The present chapter is largely based on the publication [117], but notations and figures have been revised and expanded.

\subsection{Capturing deformation and dissolving processes}

In order to understand and model materials, knowledge about their structure has to be complemented by knowledge about dynamics and functional processes. In general, it is necessary to determine the $3 \mathrm{~d}$ structure as a function of time. Ideally, a fourdimensional 4d 'material movie' would allow to observe the dynamics of the assembly and disassembly of an object at the desired spatial and temporal resolution. However, a direct reconstruction of projections from changing samples results in distorted objects with a considerable amount of artefacts like those shown in Fig. 7.1 for simulated basic transformations. During the measurement of a sinogram, the phantoms undergo any of the three affine transformations, namely translation, scaling, and shear. Additionally, a dissolving process was simulated. The resulting artefacts are similar in character and appearance to the streaks already observed for particle motion in the previous chapter. Negative values in a reconstruction from a strictly positive projection input clearly indicate these kinds of inconsistencies. But for the more complex objects considered here, non-closed borders and long-range colour gradients can also occur. In combination with the experimental challenges of high-speed tomography, including stabilisation during fast rotation and efficient handling of large amounts of data, such artefacts make it difficult to achieve the desired result quality: $4 \mathrm{~d}$ movies of a material during a chemical reaction, even a simple combustion, are almost non-existent. 


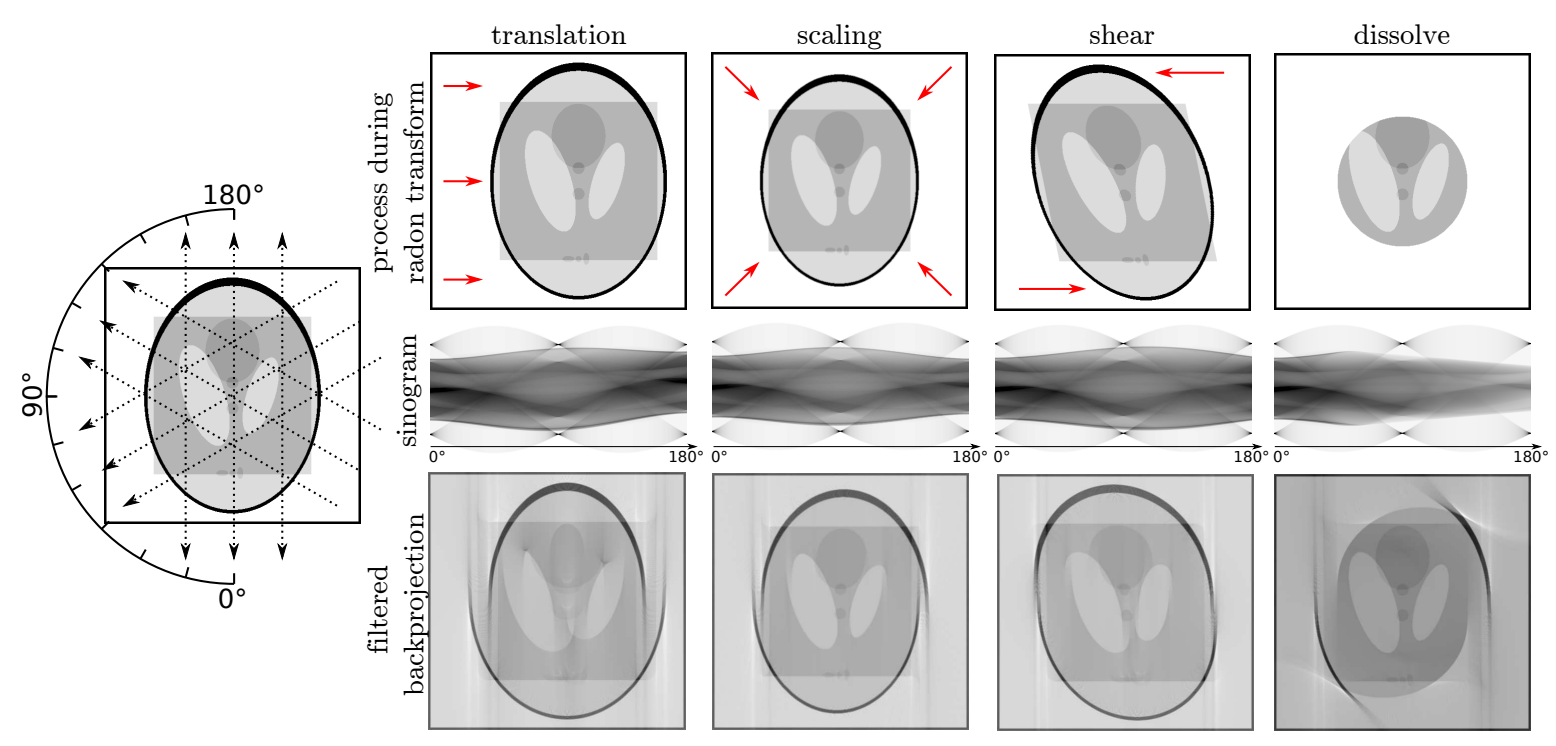

Figure 7.1: Illustration of artefacts induced by motion and dynamic processes during a tomographic measurement. The starting point $t=0$ for each simulated measurement was the image depicted on the left side with a size of $512^{2}$ pixels. Notice that the black frame is a part of the object but only the inner Shepp-Logan phantom and the grey rectangle were modified while the object was projected from $0^{\circ}$ to $180^{\circ}$ at a constant rate. The inner part was (a) linearly translated, (b) scaled with a linear decrease of the scaling factor, (c) sheared linearly and (d) 'dissolved' by multiplying a mask with linearly decreasing radius. The images in the upper row show the maximum change at the end of the tomographic scan. Each of the corresponding sinograms depicted in the middle row consists of 804 equidistant projections to satisfy angular sampling. Common filtered backprojections of the sinogram data are depicted in the bottom row. They show pronounced artefacts originating from the motion like false shapes, missing features and non-closed circles. Vertical streaks in the $0^{\circ}$ and $180^{\circ}$ direction are clearly visible in each reconstruction, since temporal and thus structural differences are largest for the corresponding projections.

Consequently, the novel experimental capabilities opened up in the recent years - notably phase contrast - can only be fully exploited if recording schemes and reconstruction algorithms are generalised from the conventional static case to meet the challenges of dynamic CT. Sophisticated regularised approaches utilise, among others, sparsity in the time evolution, but some kind of prior information and/or special sampling are required [118-120]. As detailed before, projection angle sequences with large angular increments are best suited to estimate the spatiotemporal evolution [104, 118]. A guess for the object can then be transformed in a way to best fit a sparse set of projections. To date, however, reconstruction methods are mostly restricted to global affine transformations since straight lines remain straight lines after transformation and the motion can be estimated and corrected on the projections [105, 121]. Unfortunately, these approaches are not applicable even to the simple examples in Fig. 7.1 (a) to (c), where the black border remains static during the transformation of its content. However, the approach hints at an interesting solution: If the motion is known, it can be possible to reconstruct consistent $3 \mathrm{~d}$ representations from the inconsistent projections. Tomographic reconstruction with known motion has been investigated in the literature 
[105, 121-123] and has shown success in the context of medical CT (heart beat, breathing) [124-128]. However, the experimental scenarios were restricted to pre-determined motion or rather sparse objects and over-sampled images. In such applications, the resolution is typically much smaller than the voxel size, and image artefacts have a more deteriorating influence than loss of resolution.

The method presented here addresses the requirements of dynamic CT for phasecontrast objects with micron-scale structures. It is based on deriving an approximate motion model from optical flow analysis of direct reconstructions and feeding this information into the backprojection geometry, using dynamically deformed reconstruction grids [127]. Structure and dynamics are reconstructed from the continuous stream of projection data acquired while rapidly rotating the sample, considerably decreasing the typical artefacts compromising conventional reconstruction. As a proof of concept demonstration for a $4 \mathrm{~d}$ movie of a chemical reaction, we apply this approach to monitor a match in $4 \mathrm{~d}$, burning down after laser ignition, with sufficient spatial and temporal resolution to cover the process. While individual components of the method have been used in previous work, the combination of reconstruction steps with phase contrast by free propagation has enabled unprecedented contrast and sensitivity high enough to uncover the dynamic change in the interior wood structure during the combustion. Note that grating-based phase contrast as already demonstrated for dynamic tomography [129] does not achieve the high spatial resolution required for the present application.

\subsection{Backprojection along dynamically curved paths}

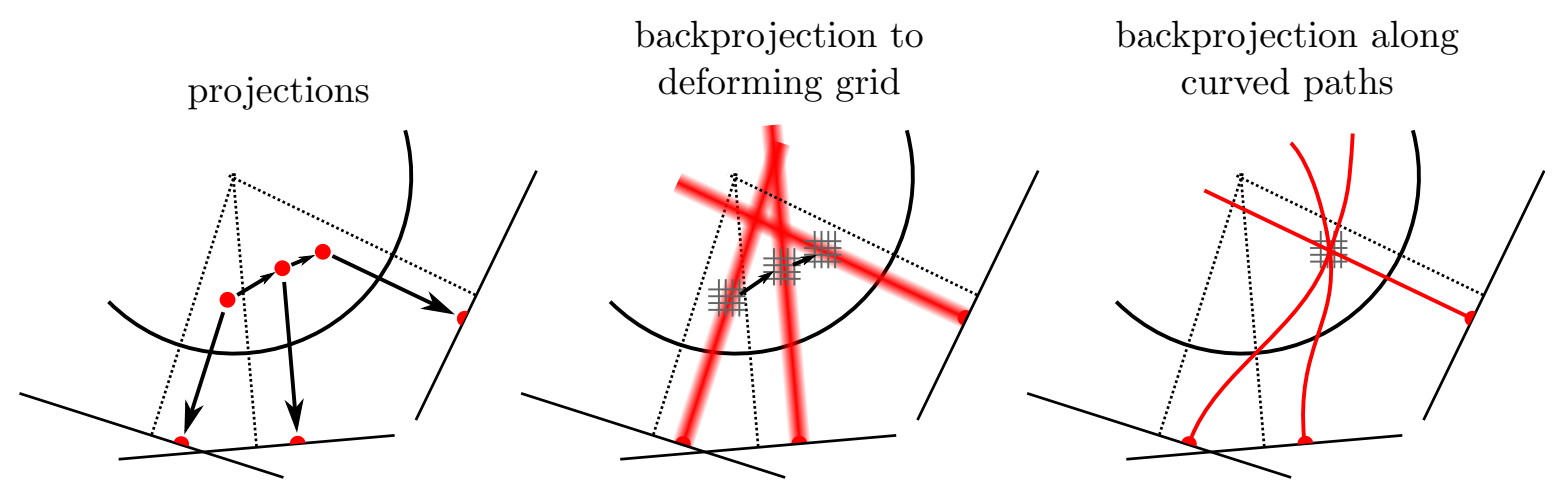

Figure 7.2: Achieving local consistency in deforming objects: A moving feature is projected for different angles (left). The direct backprojections of this signal do not intersect in a common point, preventing the reconstruction of the feature (red lines in the centre image). If the voxel grid during reconstruction locally moves along the same path as the feature during the meaasurement, it collects the signals consistently (centre). Alternatively, the backprojection paths can be dynamically curved to compensate for the motion (right). 
The basic idea of the dynamic reconstruction procedure named backprojection along $d y$ namically curved paths is sketched in Fig. 7.2. A feature moving in the volume during projection cannot be reconstructed, since the straight backprojections of the corresponding signal do not have a common point of intersection. But if the reconstruction grid locally moves along the same path as the feature in the original measurement, it consistently collects the signal from all projections. To consider all kinds of motion in the volume, the grid may have to be deformed drastically up to a point where a steady deformation is impossible. But note that the deformation does not have to follow the whole measurement over many tomographic rotations but only the reconstruction of each set of projections covering $180^{\circ}$, which should be sufficient for a wide range of samples. Alternatively, as implemented here, instead of deforming the voxel grid, the backprojection path can be deformed appropriately, crossing the different regions of the grid in the correct position and angle for local consistency. This approach makes it possible to derive high quality reconstructions from projection data that are inconsistent in the sense of conventional static tomography based on a motion model derived from the projections. This allows to compensate for general (almost arbitrary) deformations.

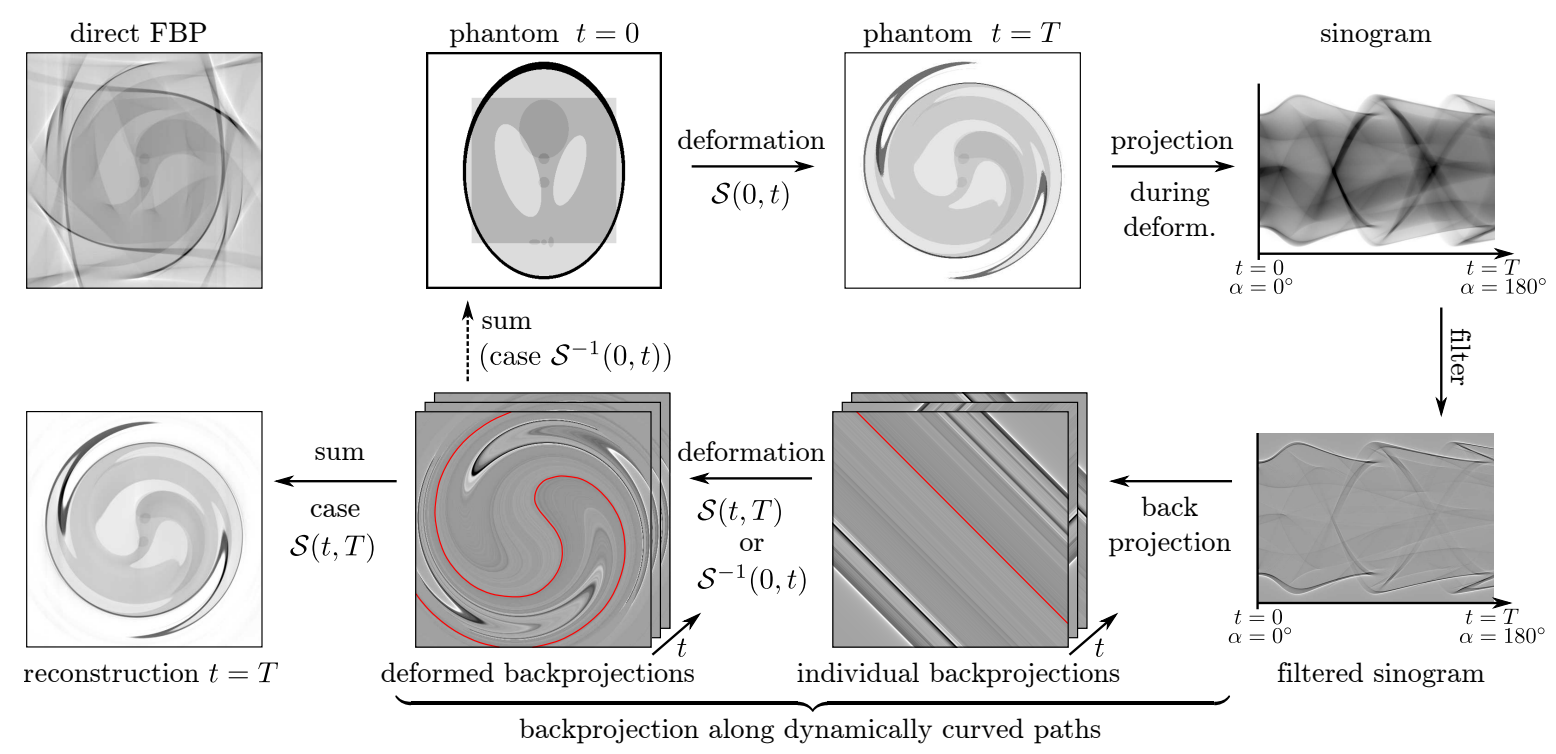

Figure 7.3: Scheme of the filtered backprojection on dynamically curved paths. The phantom is deformed as a function of time during the measurement, resulting in an inconsistent sinogram (upper row). While the conventional filtered backprojection yields a heavily distorted reconstruction (top left), the bottom row illustrates the new method, revealing a substantially improved reconstruction quality by incorporating the (known) temporal deformation. From the filtered sinogram, each individual backprojection is deformed to a different degree (see text for details). The conventional straight backprojection path is replaced by a dynamically curved path, as indicated by the highlighted red line. The superposition of all deformed backprojections results in a consistent representation of the phantom. As we show below, the motion model required for the dynamically curved paths can be estimated directly from the data. 
Figure 7.3 shows a simulation of the approach. For simplicity, we assume a $2 \mathrm{~d}$ phantom. Each projection measured at the time $0 \leq t \leq T$ originates from a deformed object $\Omega(t)=\mathcal{S}(0, t)\left[\Omega_{0}\right]$, where the operator $\mathcal{S}(0, t)$ describes the transformation from time 0 to time $t$. Here, Fig. 7.3 shows an example of a non-affine 'swirl' deformation. For reconstruction, each of the filtered projections is backprojected into the higher dimensional (2d) reconstruction space and individually transformed by $\mathcal{S}(t, T)$. The superposition of all deformed backprojections yields a consistent reconstruction of the object $\Omega(t=T)$ at the end of the recording. If the transformation $\mathcal{S}(0, t)$ can be inverted and the resulting $\mathcal{S}^{-1}(t, 0)$ is applied instead of $\mathcal{S}(t, T)$, the reconstruction results in $\Omega(t=0)$. A combination of $\mathcal{S}$ and $\mathcal{S}^{-1}$ allows for the reconstruction of $\Omega(t)$ at any given time. The backprojection and deformation steps can be merged to a single operation, i.e. a backprojection on paths which change dynamically as a function of time. In this way, the number of interpolations is reduced to that of conventional backprojection along straight paths. As illustrated, the proposed scheme enables a dramatic increase in reconstruction quality with respect to a 'naive' backprojection ignoring the dynamics. To validate this approach, numerical simulations were performed, ranging from simple motion phantoms of linear displacements to complex non-affine types of motions and achieving excellent results (data not shown). Note that the conventional ramp-filter is no longer exactly valid for the proposed scheme of deformed paths. Future design of optimised filters could possibly be based directly on the resulting density of sampling points in Fourier space or could be applied after the backprojection steps, as known from a filtered layergram reconstruction. We stress, however, that the gain in reconstruction quality is already considerable for the conventional filter, even for severe deformations as shown in the example. Notwithstanding future optimisations, this allows us to already tackle substantial experimental challenges in 4d tomography, as shown below.

Before we turn to the experiment, we have to address the experimentally relevant situation that no dynamical model for $\mathcal{S}(0, t)$ is known as prior information, but that the motion model has to be estimated from the data itself. In some special cases, the dynamical model could be derived from additional measurements, possibly enabled by traceable markers (fiducials) in the object. However, for the general case of nonperiodic and unknown motion, both structure and motion have to be derived from the projection data alone. Currently, high speed synchrotron setups are capable of recording several tomograms per second $[130,131]$, monitoring dynamics on the same time scale. The object is then mostly reconstructed using a sliding window approach, falsely assuming the structure to be static over half a revolution period. It is important to note that while the reconstruction results suffer from significant motion artefacts which impede high quality structure analysis, approximate motion models can often be derived from such directly reconstructed data. The basic idea of the current work is to use the motion model derived from the sliding window reconstruction to implement backprojection on dynamically curved paths, as introduced for known $\mathcal{S}(0, t)$ above. This approach provides a significantly higher reconstruction quality, which in turn can be used to further refine the motion model. In an iterative manner, this leads to a fundamentally improved reconstruction of the entire $4 \mathrm{~d}$ process. To demonstrate and exploit this scheme, we have performed synchrotron-based phase-contrast micro-CT of 
a burning match, with high speed data acquisition in combination with optical-flowbased motion estimation.

\subsection{The burning match experiment}

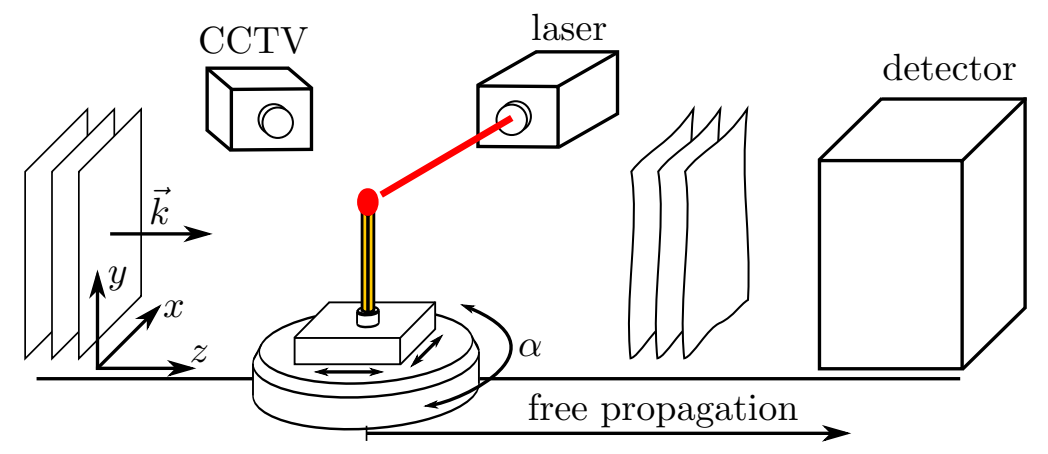

Figure 7.4: Illustration of the experiment. Setup of the TOMCAT beamline with parallel beam illumination of the match positioned at the centre of a fast rotating stage. The match was ignited by a laser system and observed by a CCTV camera. The detection system was positioned $228 \mathrm{~mm}$ behind the object.

The experiment was carried out at the TOMCAT beamline of the Swiss Light Source [130]. The setup is sketched in Fig. 7.4. A parallel beam with a photon energy of $E_{p h}=20 \mathrm{keV}$ and a flux of about $10^{12} \mathrm{ph} / \mathrm{s} / \mathrm{mm}^{2}$ was used to illuminate the object positioned in the centre of a fast rotating stage. The detection system was positioned $228 \mathrm{~mm}$ behind the object. X-rays were converted to optical signals by a $150 \mu \mathrm{m}$ thick LAG:Ce scintillator, which was magnified by a factor of 3.73 to a high speed CCD Camera (PCO Dimax), resulting in an effective pixel size of $2.95 \mu \mathrm{m}$. Matches made of larch wood and impregnated with paraffin wax were obtained from a local supermarket. The matches were cut to a length of approx. $8 \mathrm{~mm}$ and the wooden part directly under the phosphorous head was chosen as the region of interest. For the recordings, the matches were first aligned to fit horizontally into the field of view of the camera under all angles using position motors above the rotation stage. Then, the rotation speed was increased until the measuring speed was reached. The matches were ignited by an IR laser system [132] and observed with an optical CCTV camera. The acquisition was started manually after the ignition flame appeared on the live monitoring screen. We did not observe an influence of the rotation on the flame and burning speed. Several burning processes were recorded with rotation speeds from $1.25 \mathrm{~Hz}$ up to $3.5 \mathrm{~Hz}$, resulting in 2.5 up to 7 full tomograms per second. However, the duration of the measurement was limited by the internal memory of the camera system of $36 \mathrm{~Gb}$. An acquisition time of $1 \mathrm{~ms}$ for each projection allowed the recording of the entire burning process in 18800 images (and thus 18.8s). At the slowest rotation speed of $1.25 \mathrm{~Hz}, 401$ angles per sinogram were sufficient to reconstruct details and follow the process. Fewer images per sinogram led to a dramatic decrease of resolution. Thus faster rotations require shorter acquisition times, resulting in a lower signal to noise ratio not outweighed by the decrease in motion artefacts. Higher rotation speeds had 
the further drawback that the entire burning process could not be covered, since the maximum possible number of images was reached more quickly. Most importantly, the movies recorded at a high rotation speed in the testing phase proved that all processes observed on the resolution scale of the experiment were slow enough to be well captured by the slower recordings. Thus, we chose the measurements with a rotation speed of $1.25 \mathrm{~Hz}$ for our subsequent data evaluation.

\subsection{Data analysis}

This section follows the reconstruction of the object and its dynamics from the data. First, the raw data was corrected for the artefacts induced by the illumination. In a second step, the phase retrieval was performed, followed by the independent tomographic reconstruction with all improvements.

\subsubsection{Projection retrieval and alignment}

A raw detector image with a size of $1200 \times 1116$ px is shown in Fig. 7.5 (a). It is affected by artefacts originating from a spatially and temporally inhomogeneous illumination. These artefacts were accounted for by the usual raw data corrections: First, all images were dark-field corrected and divided by the mean of 200 flat field images of the empty beam. Due to the short acquisition time, however, flickering of the illumination was not averaged out. Notably, we observed pronounced vertical shifts and structural changes in the illumination, most probably originating from vibrations of the beamline's monochromator. They result in horizontal stripe artefacts after the flat-field correction, as depicted in Fig. 7.5 (b). This type of artefact is usually not visible in conventional measurements, where the acquisition time is large enough to average out all vibrations. Since the undesired structures appeared to be ordered predominantly along the horizontal axis and in a narrow band of spatial frequencies, we were able to remove them by a Fourier filter derived from the flat-field images as follows: Each flat-field image was divided by the mean of all 200 flat-field images. Without any vibration, this would result in homogeneous images, but for the present data, pronounced horizontal intensity gradients resulting from the vibrations appeared. The images were Fourier transformed and their modulus was averaged. By applying a threshold, the resulting average was transformed into a binary mask discriminating between spatial frequencies from the background artefacts and frequencies that had to be attributed to the sample. All projections of the match were filtered in Fourier space using this mask, removing only structures already appearing in the flat-field images due to vibrations. As a result, the artefacts disappeared almost completely without visually impairing the match's projection.

Furthermore, we have observed a degradation of the scintillator screen, possibly due to the heat during the ignition process. After the ignition, ring-like structures (see Fig. 7.5 (b)) appeared in all projections which were not visible in flat-field images recorded before. These rings slowly grew during the measurement. They could be removed 


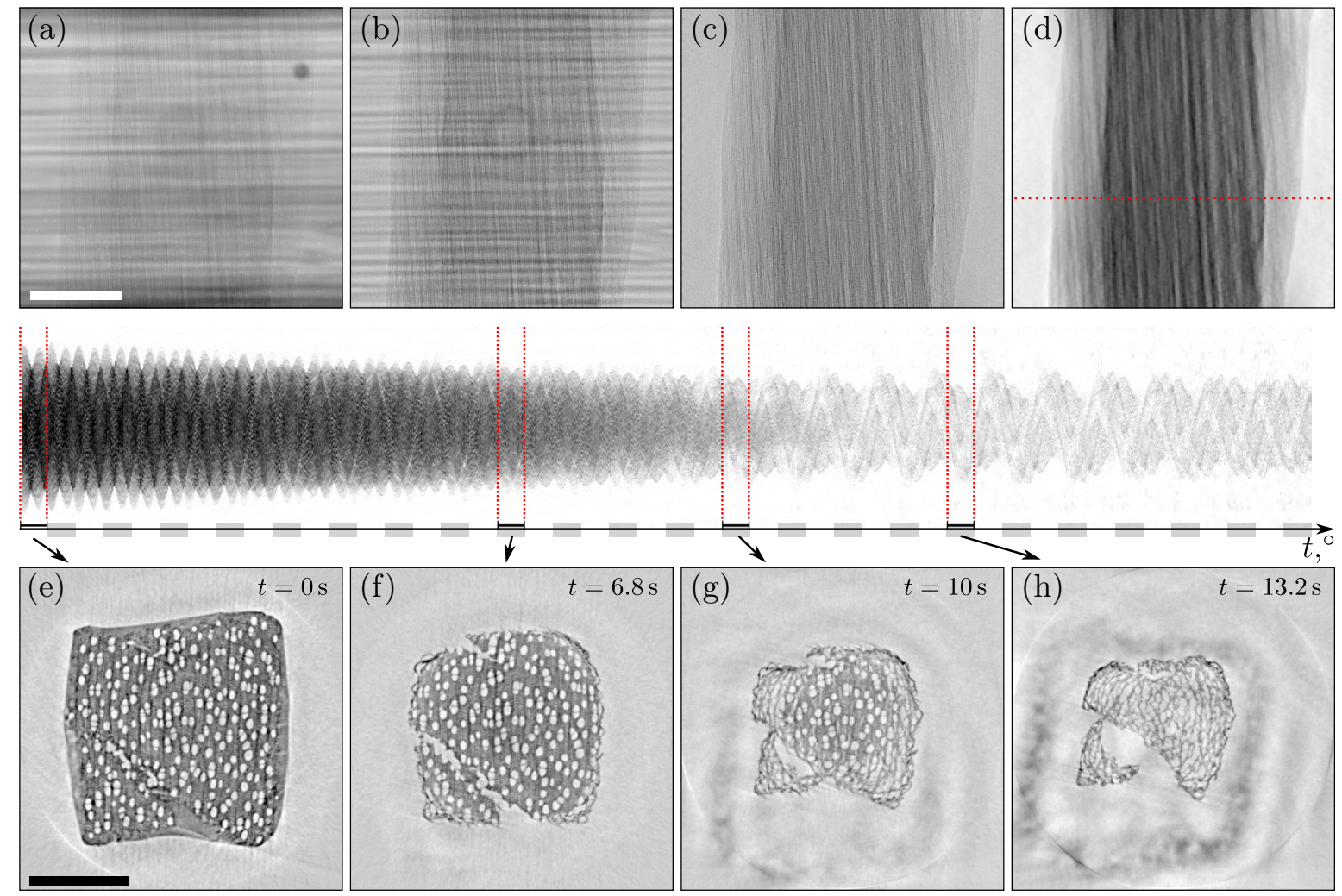

Figure 7.5: Data treatment from the raw detector images to the 3 d structure. A typical detector image with an exposure time of $1 \mathrm{~ms}$ is depicted in (a). After the flatfield correction, horizontal stripes and a ring-structure still impair the data, as shown in (b). Both artefacts could be removed by Fourier filters to yield the cleaned image in (c). Contrast was enhanced significantly by TIE-based phase retrieval (d). The sinogram in the centre of the figure was extracted from all 18800 reconstructed projections at the position of the red dashed line. A shrinking of the structure and a loss of material can be observed by a decrease in photon absorption. The bottom row depicts tomographic reconstructions of the highlighted sinogram segments, each consisting of 401 equidistant projections. The shape, features of the wooden structure and the stages of the burning process can be clearly identified. Nevertheless, the reconstructions show motion artefacts such as streaks and non-closed shapes in detail. The scale bars represent a width of $1 \mathrm{~mm}$.

sufficiently by averaging the projections from $-180^{\circ}$ to $180^{\circ}$ around each projection. The ring-structure could then be isolated by an adapted high-pass-filter, and was subtracted from the corresponding image. The results, as depicted exemplarily in Fig. 7.5 (c), show very little absorption contrast of the wood cell wall. For the chosen photon energy range and biomolecular matter such as cellulose, $\beta$ is on the order of $10^{-10}$, while $\delta$ is up to three orders of magnitude larger. Since the parallel beam illumination provided a sufficiently high degree of coherence, edge enhancement is clearly visible 
in the projection and near-field phase retrieval could be used to improve the image contrast. To this end, the simple parabolic Fourier filter

$$
\tilde{u}\left(\nu_{\perp}\right)=\frac{1-\exp \left[-\varepsilon \cdot\left(\nu_{\perp}^{2}\right)\right]}{\nu_{\perp}^{2}} .
$$

defined in Eq. (5.32) has been applied with the regularisation parameter $\varepsilon=0.001$, preserving a certain degree of edge-enhancement beneficial for motion detection. This contrast enhancement ultimately enables the fast measurements as reported here.

The outcome of performing all corrections together with the phase retrieval is shown in Fig. 7.5 (d), next to a sinogram extracted from the stack of all corrected images of a recording. It clearly shows the shrinking of the structure as well as a decrease of signal intensity, originating from the loss of mass during burning. Intervals of 401 images were selected from the sinogram to compute subsequent tomographic reconstructions. Filtered backprojections of four intervals are shown in the bottom row of Fig. 7.5. To ensure a well-aligned vertical rotation axis in the centre of the projections, which can in general not be sufficiently realised in an experiment, all images were rotated by the misalignment angle (angle between the determined axis of rotation and the detector columns). Additionally, the distance of the determined axis of rotation to the centre of all images was corrected for. Both parameters (angle and offset) were determined by comparing two projections from opposite sides with an angular distance of $180^{\circ}$. For static tomography, the automatic determination of both parameters in general warrants high quality reconstructions without any sign of misalignment. Contrarily, for dynamic tomography, a further refinement may be required. This is due to the fact that in a series of subsequent reconstructions, even the smallest deviations from the original values result in a minute sub-pixel shift of subsequent reconstructions. This misalignment is sensitively registered by the human eye as a vibration in the time series, while it would not be detectable in a single reconstruction. Therefore, both parameters have been refined manually.

\subsubsection{Optical flow estimation}

As shown in Fig. 7.5, the described combination of corrections applied to each projection, phase retrieval and corrections of the tomographic axis resulted in a temporal series which covered the burning process remarkably well already. Upon closer inspection, however, many details especially at the moving borders are blurred by the intrinsic dynamics of the burning process, despite the relatively fast data acquisition. In the following, this issue is addressed by utilising backprojection on dynamically curved paths as introduced above. To obtain an estimation of the motion, we performed optical flow (OF) analysis [133]. Since structure and dynamics of the match vary only slowly parallel to the axis of rotation, it was sufficient to estimate the motion in the perpendicular dimensions only, considerably reducing the computational effort. In the time series, each subsequent pair of slices perpendicular to the rotation axis was compared as illustrated in Fig. 7.6 (a). Generally speaking, the first slice was broken up into small patches, followed by searching for the best registration within the subse- 

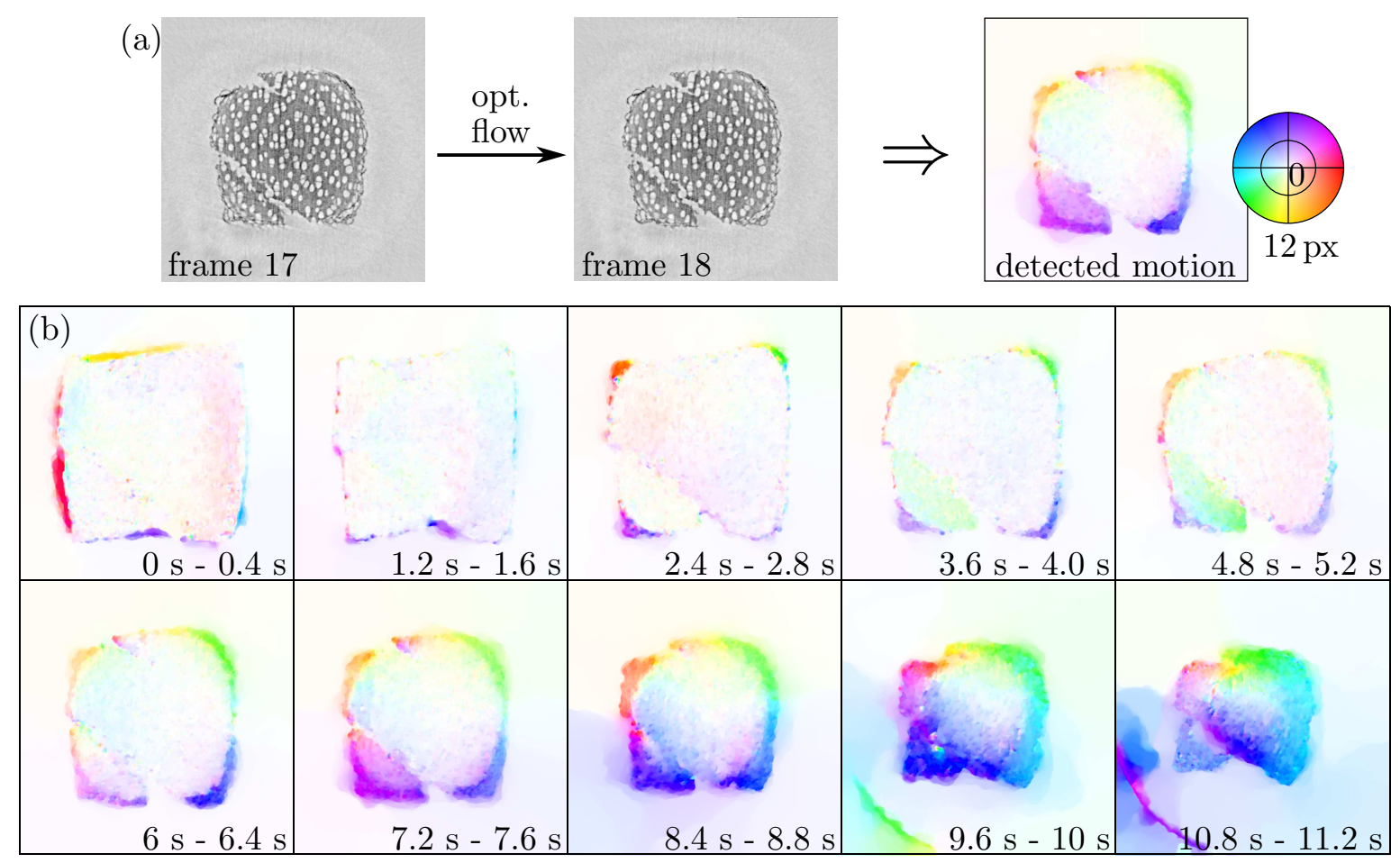

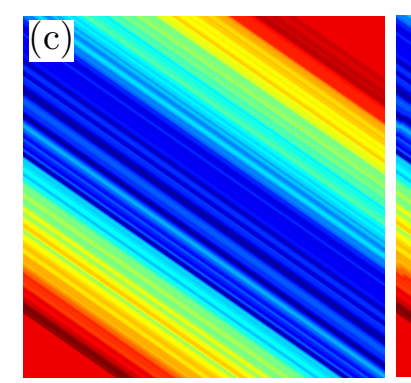

conventional backprojection

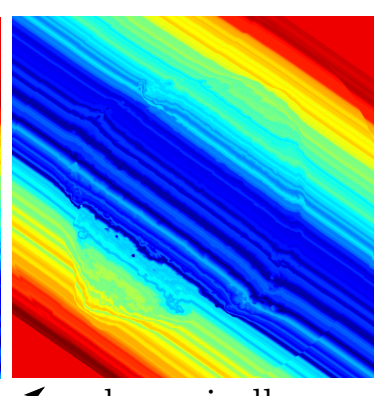

dynamically curved paths

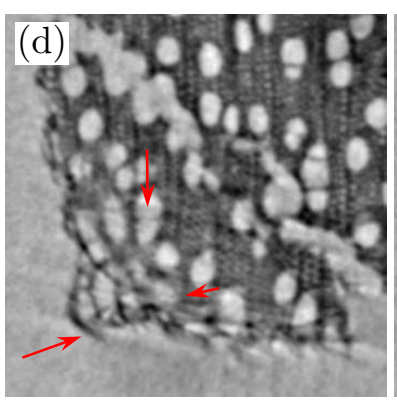

conventional reco.

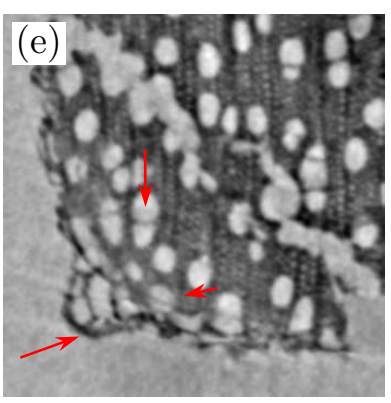

improved reco.

Figure 7.6: Illustration of the motion estimation for the subsequent backprojection along curved paths. Two successive reconstructions (a) are analysed by an optical flow algorithm. The depicted result shows the shrinking at the borders of the match. The colour indicates the direction of motion as shown by the colour wheel, the saturation gives the motion amplitude with a maximum of $12 \mathrm{px}$ for all images shown. The image series in (b) gives an overview of the dynamics of the entire burning process, showing the optical flow result for every third frame. Using the motion information, the reconstruction is then carried out by backprojection on dynamically curved paths, as illustrated in (c) with a magnification of the motion amplitude by a factor of 10 for better visibility. A significantly improved reconstruction quality is observed, as seen for the fine object details resolved in (e), compared to the result of (d) for a conventional direct FBP reconstruction.

quent image to obtain a map of local displacement vectors. Here, we used the powerful OF implementation from [133]. To cross-check the correctness of the detected motion, the second image was geometrically backtransformed to the shape of the preceding one (warping), using the previously obtained displacement. Computationally, this step was performed by inverse mapping as implemented in MATLAB's interp2 function 
[133]. Visual comparison of the difference between original and backtransformed image showed a good agreement throughout. The motion of the entire burning process in one slice is summarised in Fig. 7.6 (b). In the beginning, only the thin outer layer shows shrinking. As the burning horizon moves to the centre of the wooden structure, the shrinking layer of burned wood becomes thicker until the whole structure is burned. Because of the flame's progress from top to bottom, the already burnt structure does not stay constant but in the end shows large and rapid displacements as tensions in the match change. This collapse-like situation is the only one whre the motion estimation failed.

\subsection{3 $3 \mathrm{~d}$ reconstruction}

Assuming a linear transformation within each tomographic interval (401 images over $180^{\circ}$ ) as a first order motion model, a reconstruction was computed as follows: For a given angle (point in time), a tomographic reconstruction from the previous and from the following $180^{\circ}$ range of projections was computed. This is particularly beneficial for streak-affected data since the orientation of the streak motion artefacts depends on the selection of the starting angle and is the same in both reconstructions. OF analysis was then performed to derive a motion model. If, for example, the object undergoes a theoretical linear global shift from $x(t=0)=-L$ to $x(t=T)=L$ during the $360^{\circ}$ scan, both direct reconstructions would be perturbed in similar ways. The main difference between both reconstructions would be a shift about $L$ against each other, which is the same shift magnitude which the object underwent during the recording of $180^{\circ}$. Hence, the detected magnitude of motion can be used directly without rescaling for the subsequent application of filtered backprojection on dynamically curved paths, which is used to 'sharpen' the reconstruction of the second set of projections at the given point in time. Fig. 7.6 (c) shows an exemplary backprojection with the deformation enlarged by a factor of 10 for better visibility. With this approach, the motion artefacts were suppressed, significantly improving the reconstruction quality (compare, for example, the slices shown in Fig. 7.6 (d) without correction and (e) with correction). Both images depict an enlarged region of the object, reconstructed from the same projections. The direct reconstruction in (d) shows artefacts especially at the outer border of the match including non-closed disrupted structures and clearly visible streaks. In the motion based reconstruction, these artefacts are reduced considerably. New details in the wooden structure become visible as indicated. At this point, the entire process of motion estimation and correction can be iterated with the higher quality reconstructions. However, for the match presented here, a single iteration appeared to be sufficient. Based on the proposed dynamic backprojection approach, the dynamics of the burning process can be investigated with high image quality and at high spatial and temporal resolution. The $4 \mathrm{~d}$ nature and amount of data to analyse is illustrated in Fig. 7.7, showing the rendered 3d structure at five different times during the process. A high quality movie of the dynamics is available as online material in [117], including single slices as well as a render of the entire structure. 

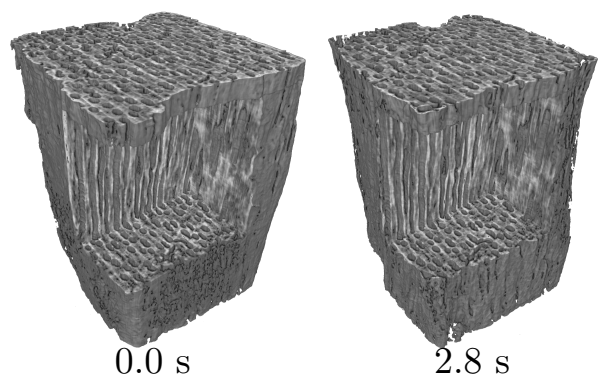

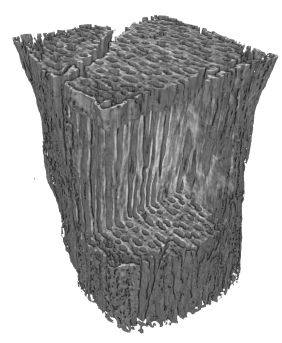

$5.6 \mathrm{~s}$

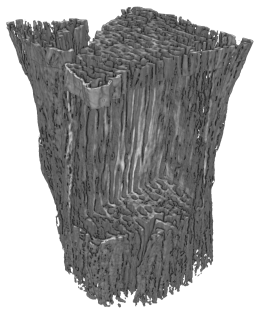

$8.4 \mathrm{~s}$

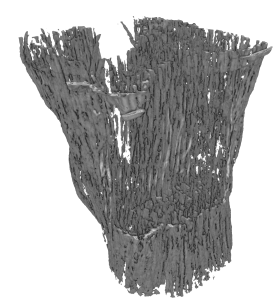

$11.2 \mathrm{~s}$

Figure 7.7: Rendered 3d structure of the burning match at different times.

\subsection{Summary and outlook}

In summary, this chapter presents a novel combination of techniques for $4 \mathrm{~d}$ tomography, backprojection along dynamically curved paths in combination with optical flow analysis. This optimised reconstruction from the projection data stream provides material movies with considerably reduced motion artefacts and at spatial and temporal resolution sufficient to monitor micron scale structural changes during combustion. As a proof-of-concept, we have studied the burning process of a match in $4 \mathrm{~d}$, which after a hundred years of using matches in everyday life has certainly never before been studied in $4 \mathrm{~d}$. Choosing wood as a sample, and a simple burning as a reaction, the example also covers one of the first materials and material processes used by mankind.

Several experimental and instrumental aspects were of particular importance for this work, notably the fast cameras. Regarding detection and data transfer, the GigaFRoST technology at the Swiss Light Source will provide even higher acquisition speeds at a rate of up to $8 \mathrm{~GB} / \mathrm{s}$ [131]. To record the changes of the cellular wood structure (tracheids with cell walls and lumen) during the combustion, phase contrast was essential. It is much more sensitive to softer materials and smaller structures than absorption contrast alone. The increase in contrast allows for shorter exposure times and thus faster measurements which facilitate the optical flow analysis. Furthermore, the raw data treatment and filtering of illumination artefacts was also a necessary step to reach the level of data quality where the phase retrieval and optical flow analysis could be put to the test successfully. The role of empty beam division and aberrations in the illumination wave front has previously been addressed in [113].

Several improvements to the present schemes can be directly envisioned: (i) Curved path backprojection and motion estimation can be iterated, as mentioned above. (ii) The motion estimation can include higher orders of interpolation. For example, the second order acceleration of the motion can be investigated by starting the calculation of the optical flow at a slightly different angle and comparing the sets of optical flow images. (iii) The concept of dynamically curved paths can be easily extended from $2 \mathrm{~d}$ slices to $3 \mathrm{~d}$ volumes. (iv) Finally, the concept of backprojection along dynamically curved paths can be combined with the regularised models for dynamic CT, based on prior information.

Using this combination of phase contrast recordings, motion estimates based on optical flow and subsequent backprojection along appropriate dynamically curved paths, it 
should become possible to achieve the spatial and temporal resolution required for the observation of material processes on the nanoscale. To this end, the parallel beam illumination used here has to be replaced by a cone beam geometry with nanoscale secondary focal spot size [58] and intrinsic geometric magnification. 



\section{Summary and outlook}

This work addresses a variety of aspects of the field of time-resolved X-ray phasecontrast tomography. First, a combination of static tomography with phase retrieval is discussed, and a powerful new reconstruction method is presented: In the special but relevant case of a weak object, phase retrieval can be carried out entirely in $3 \mathrm{~d}$, considerably improving the reconstruction quality. To this end, an artefact-free numerical implementation of wave field propagation is introduced which scales well towards higher dimensions. Challenges in the treatment of dynamic samples are discussed next and the deep insight which time resolved tomography offers is demonstrated on the example of sedimenting $\mathrm{SiO}_{2}$ micro-spheres in a water-filled capillary. An approach to overcome limitations in spatial and temporal reconstruction quality by incorporating a motion model into the reconstruction procedure is demonstrated on the example of a burning wooden matchstick.

Both experiments are based on fast and sensitive high-resolution X-ray tomography. Without the need for scanning, this is accomplished by propagation based near-field holography. Therefore, a whole chapter is dedicated to the foundations and implementation of wave-field propagation, presenting both an angular-spectrum based and a real-space convolution based approach. This includes some very fundamental notes on numerical sampling und aliasing problems, with the proposition of an intuitive method to remove the corresponding artefacts: Usually, the propagation can be implemented by a real-space convolution with a kernel that corresponds to a slightly modified spherical wave, thus transporting information from one pixel to the whole positive half-space. While this is justified for a point source, the angle of radiation originating from a finite pixel has to be limited. By properly modifying the convolution kernel, sampling artefacts are completely removed, thus eliminating the need for conventional costly zero-padding.

An important application of such numerical propagation is the problem of phaseretrieval. A new approach to address this challenge was presented based on the novel concept of propagating entire $3 \mathrm{~d}$ objects, combining phase retrieval with tomography. The common scheme of first retrieving the phase information of all projections individually followed by an inverse Radon transform is reversed. Instead, a 3d "intensity volume" is computed from the measurements, which then serves as a constraint in an iterative algorithm working directly on $3 \mathrm{~d}$ volumes to reconstruct the object structure from all projections in parallel. A big advantage of this new reconstruction method is the possibility to enforce a new class of constraints in $3 \mathrm{~d}$, both in the object and the detection plane. Since the inverse Radon transform is performed only once, a tremendous enhancement in reconstruction speed is obtained with respect to previous schemes of combined iterative phase retrieval and tomographic reconstructions. At the same time, the $3 \mathrm{~d}$ propagation method preserves the essential benefits of tomographic consistency, which is intrinsically enforced by the $3 \mathrm{~d}$ scheme and was found to stabilise phase re- 
trieval with otherwise under-determined data. In combination with non-negativity, a requirement for holographic imaging, the constraints can be sufficient to reconstruct $3 \mathrm{~d}$ objects without any further a priori knowledge.

In addition to revealing static $3 \mathrm{~d}$ structures with high sensitivity, propagation-based phase contrast enables high acquisition rates with still sufficient signal to noise ratio to investigate the $3 \mathrm{~d}$ dynamics of micro-scale samples. This is demonstrated on sedimentation, one of the oldest technical processes for the separation of particles from each other and from liquids, which is still far from being completely understood. Following the trajectories of monodisperse $\mathrm{SiO}_{2}$ microspheres with a diameter of $2 \mu \mathrm{m}$ in a waterfilled rectangular capillary with an edge length of $50 \mu \mathrm{m}$, both the sedimentation and Brownian motion of thousands of spheres can be observed in great detail. Complex paths with large lateral displacements, particles rising against gravity in intertwined and dynamically changing water currents as well as effects at the interface to the already settled particles can be observed directly. Notably, the measurement was carried out in a synchrotron-based micro-CT setup with X-ray waveguide illumination, whose high sensitivity and resolution are compatible with many interesting biological samples, demonstrating the possibility to reveal fast dynamics in this challenging environment. As a result of movement in the sample during recording, characteristic motion-artefacts occur in conventional reconstructions. While this is shown to be acceptable when determining the positions of identical objects such as spheres, the artefacts significantly impair the reconstruction of unknown sample structures. The second experiment shows a way to overcome this long-standing limitation: A novel combination of techniques for $4 \mathrm{~d}$ tomography, namely backprojection along dynamically curved paths based on a motion model obtained by optical flow analysis, allows monitoring micron-scale structural changes with a temporal resolution considerably smaller than the acquisition time of a sinogram. We have successfully applied this scheme to resolve the combustion of a wooden matchstick with a level of fidelity unattainable by conventional methods.

The results of this work suggest some further directions of investigation. Since we have seen the advantages of combining phase retrieval and tomographic reconstruction, an obvious but non-trivial next step would be the inclusion of motion retrieval in the reconstruction process. This would allow utilising new kinds of constraints, e.g. the detection of high accelerations in one area might restrict the local material composition. At the moment, the handling of such large data sets with tens of thousands of high resolution projections is still challenging, however, it seems likely that further advances in data processing will soon enable more complex iterative procedures. One could envision algorithms minimising a global cost function that punishes deviations of a $4 \mathrm{~d}$ guess from the measured data with degrees of freedom that allow a dynamic within certain physically motivated boundaries. On the other hand, sequential schemes as demonstrated on the $\mathrm{SiO}_{2}$ particles can also be the solution of choice, offering a highly adaptable approach to the processes. Key components of such a unified algorithm have already been introduced in this work: from numerical implementations of artefact-free propagation, even in 3d, to advanced concepts of motion estimation and correction. Besides this algorithmic development, continuous improvements of the instrumentation at synchrotrons, the development of free electron lasers, and improved data processing are promising a vibrant and exciting future for time-resolved phase contrast tomography. 


\section{Appendix}

\subsection{Numerical backprojection of $2 \mathrm{~d}$ images}

The main part of the implementation of a backprojection is explained in this section. The program is optimised for the CPU-based workstation at the Institute for X-ray Physics, with 20 physical cores and a memory of about $512 \mathrm{MB}$ (ECC RAM). The routine is based on the parallel (back)projection of single voxels whose edge length is identical to that of the projection pixels, as explained in Chap. 3.3. To this end, the function backproject requires pointers to the reconstruction grid grid_ptr, the stack of projections prop_ptr and the projection angles thetas (in degrees), as well as the height $\mathrm{nz}$ and the width (and depth) $\mathrm{nx}$ of the projections and the volume, the number of projections angles in the stack and the shift of the rotation axis with respect to the centre of the volume. The rotation axis is parallel to the $\mathrm{nz}$ direction.

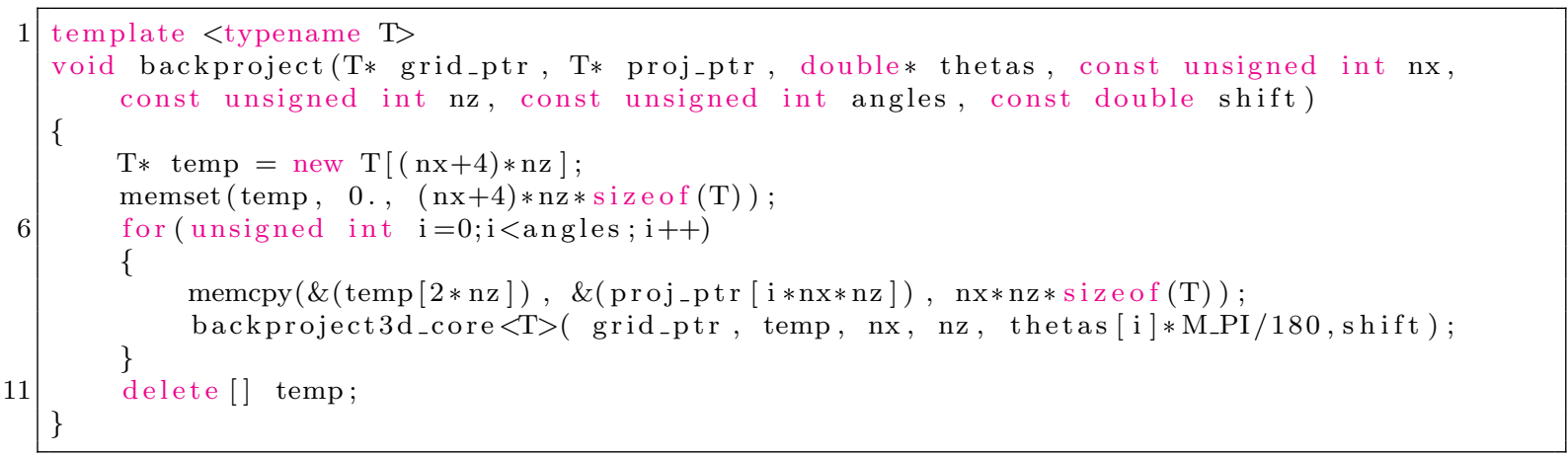

Here, each projection is copied into the larger image temp with the additional two pixel rows each on the left and right side equal zero. This allows the parallelised main routine backproject3d_core to save expensive if-queries. The voxels are distributed equally to the CPU threads. Each voxel column parallel to the rotation axis is projected onto a maximum of three projection image columns. The weighting factors are calculated according to Eq. (3.22) and each voxel adds the values of the corresponding pixels of the projection images, weighted with these factors to its own value:

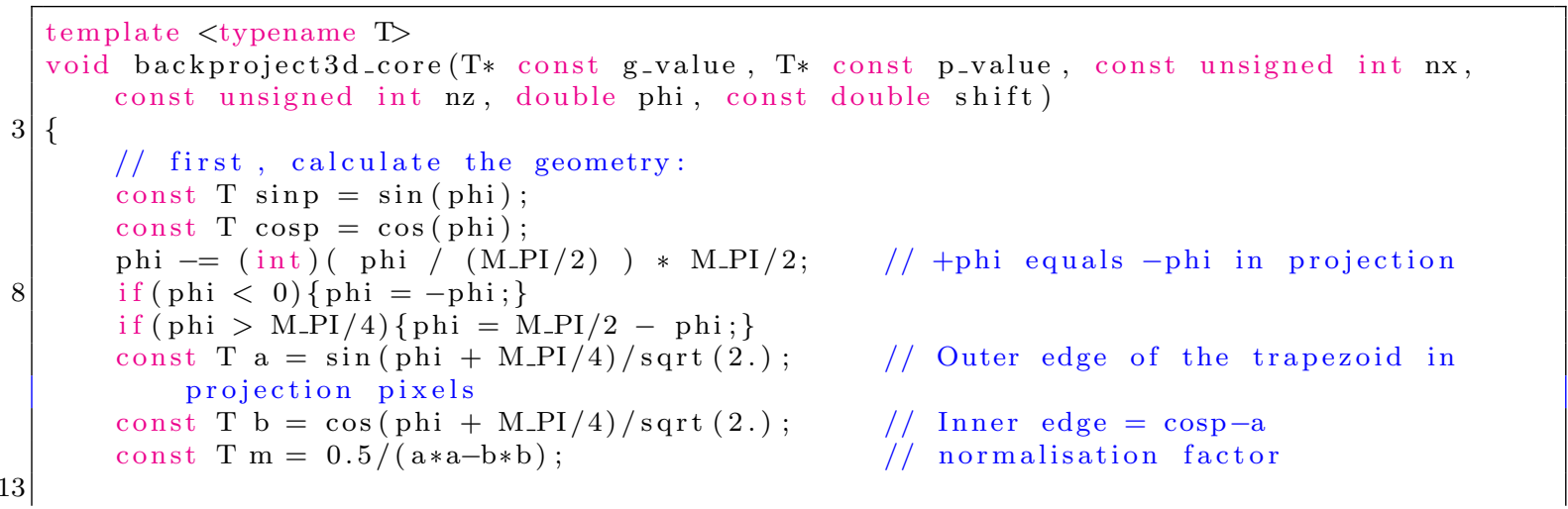




\section{Appendix}

// parallel part of the program \#pragma omp parallel

\{

68 int threads = omp_get_num_threads (); // get number of threads

int id = omp_get_thread_num (); $\quad / /$ get individual number of this thread

$\operatorname{var64}$ start $=\mathrm{id} *((\mathrm{nx} * \mathrm{nx}) /$ threads $) ; \quad / /$ distribute the voxel equally to the threads

$\operatorname{var} 64$ end $=(\mathrm{id}=$ threads -1$) ?(\mathrm{nx} * \mathrm{nx}):((\mathrm{id}+1) *((\mathrm{nx} * \mathrm{nx}) /$ threads $))$;

unsigned int eol $=($ start $/ \mathrm{nx}+1) * \mathrm{nx}-1$;

// index of the next 'horizontal',

$\mathrm{T} \operatorname{pos} 0=0.5 *((\mathrm{nx}-1) *(\operatorname{sinp}-\operatorname{cosp})+\mathrm{nx})-\operatorname{shift}-(\operatorname{start} / \mathrm{nx}) * \operatorname{sinp} ; / /$

projection of the centre of the first voxel in the starting column

$\mathrm{T} \operatorname{pos}=\operatorname{pos} 0+(\operatorname{start} \% \mathrm{nx}) * \operatorname{cosp} ; \quad / /$ exact position of the centre of the first voxel in the projection

$\mathrm{T}$ w1, w2, w3;

// weighting factors for the three columns

int $\mathrm{lb}=($ int $)(\operatorname{pos}-\mathrm{a}+4096)-4096+2 ; \quad / /$ diskrete position in projection $(+2$ die to padding $)$

$\mathrm{T}$ dist $=1 \mathrm{~b}-$ pos -1 ;

var64 index;

for $(\operatorname{var} 64 \quad \mathrm{i}=$ start $; \mathrm{i}<$ end ; i + +)

\{

if $(\mathrm{lb}>=0$ \&\& $\mathrm{lb}<\mathrm{nx}+2)$

values from projection images

// project voxels and collect

\{

if $($ dist $>-b)\{$

$\mathrm{w} 1=(\mathrm{dist}>\mathrm{b}) ? 1-\mathrm{m} *(\mathrm{a}-\mathrm{d}$ ist $) *(\mathrm{a}-\mathrm{d}$ ist $): \operatorname{dist} /(\mathrm{a}+\mathrm{b})+0.5$;

$\mathrm{w} 2=1-\mathrm{w} 1$;

index $=1 \mathrm{~b} * \mathrm{nz}$;

for $(\operatorname{var} 64 \quad \mathrm{j}=\mathrm{i} * \mathrm{nz} ; \mathrm{j}<\mathrm{i} * \mathrm{nz}+\mathrm{nz} ; \mathrm{j}++)\{$ $\mathrm{g}_{-}$value $[\mathrm{j}]+=\mathrm{p}_{-}$value $[\mathrm{index}] * \mathrm{w} 1+\mathrm{p}_{-}$value [index+nz] $* \mathrm{w} 2$; index ++ ;

\} els e \{

$\mathrm{w} 1=\mathrm{m} *(\mathrm{a}+\mathrm{d}$ ist $) *(\mathrm{a}+\mathrm{d}$ ist $)$;

if $(\mathrm{d}$ ist $+1>\mathrm{a})$

\{

$\mathrm{w} 2=1-\mathrm{w} 1$

index $=1 \mathrm{~b} * \mathrm{nz}$;

for $(\operatorname{var} 64 \quad \mathrm{j}=\mathrm{i} * \mathrm{nz} ; \mathrm{j}<\mathrm{i} * \mathrm{nz}+\mathrm{nz} ; \mathrm{j}++)\{$

$\mathrm{g}_{-}$value $[\mathrm{j}]+=\mathrm{p}_{-}$value $[$index $] * \mathrm{w} 1+\mathrm{p}_{-}$value [index+nz] ${ }^{\mathrm{w}} 2$;

\} index ++ ;

else

\{

$\mathrm{w} 2=1-\mathrm{m} *(\mathrm{a}-\mathrm{d}$ ist -1$) *(\mathrm{a}-\mathrm{d}$ ist -1$)-\mathrm{w} 1$;

$\mathrm{w} 3=1-\mathrm{w} 1-\mathrm{w} 2$;

index $=1 \mathrm{~b} * \mathrm{nz}$;

for $(\operatorname{var} 64 \quad \mathrm{j}=\mathrm{i} * \mathrm{nz} ; \mathrm{j}<\mathrm{i} * \mathrm{nz}+\mathrm{nz} ; \mathrm{j}++)\{$

$\mathrm{g}_{-}$value $[\mathrm{j}]+=\mathrm{p}_{-}$value $[$index $] * \mathrm{w} 1+\mathrm{p}_{-}$value $[$index+nz $] * \mathrm{w} 2+$ p_value [index+nz+nz] $*$ w 3 ; index ++

\}

if $(\mathrm{i}<\mathrm{eol})\{$

dist $-=\operatorname{cosp}$

if $(\mathrm{d}$ ist $+\mathrm{a}>1)\{\mathrm{lb}--;$ dist $--;\}$

else if $(\mathrm{dist}+\mathrm{a}<=0)\{\mathrm{lb}++; \mathrm{dist}++;\}$ 


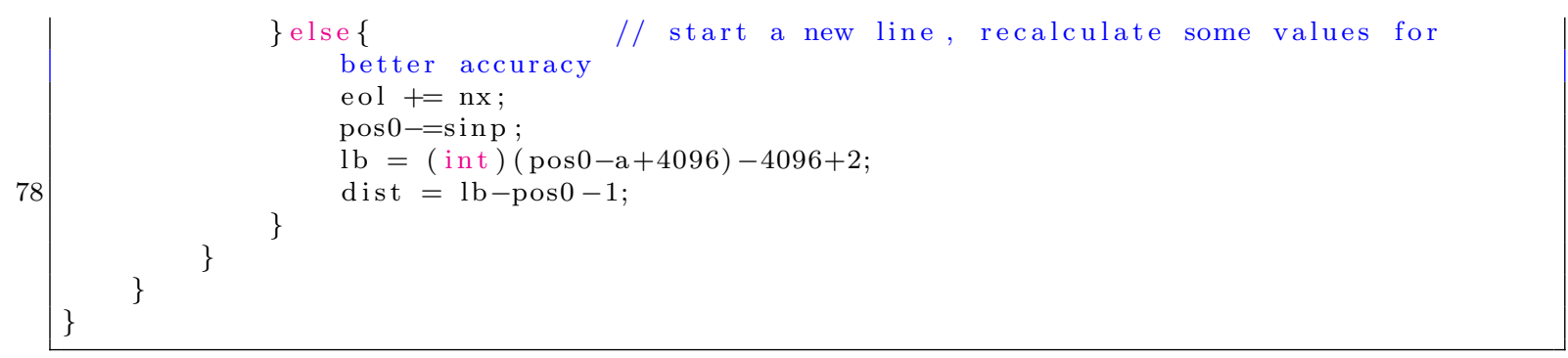

\subsection{Convolution-based numerical propagation}

A basic Matlab implementation of the convolution-based 2d propagation with the kernel limited to the well-sampled region according to Chap. 4.3 is presented in the following. As parameters, the function requires an image img to be propagated, the pixel size px_size, wavelength lambda and propagation distance dist in the same arbitrary units. Optional arguments are a lateral shift of the propagation kernel out_shift in units of pixel, and the amplitude of the illumination wave virtual_padd. By default, the image is assumed to be surrounded by perfectly absorbing material. This can be changed by the virtual_padd value to the signal of an infinitely extended plane wave with the given (complex) amplitude at the starting distance. The function returns the propagated image res as well as the propagation kernel pmap in real space:

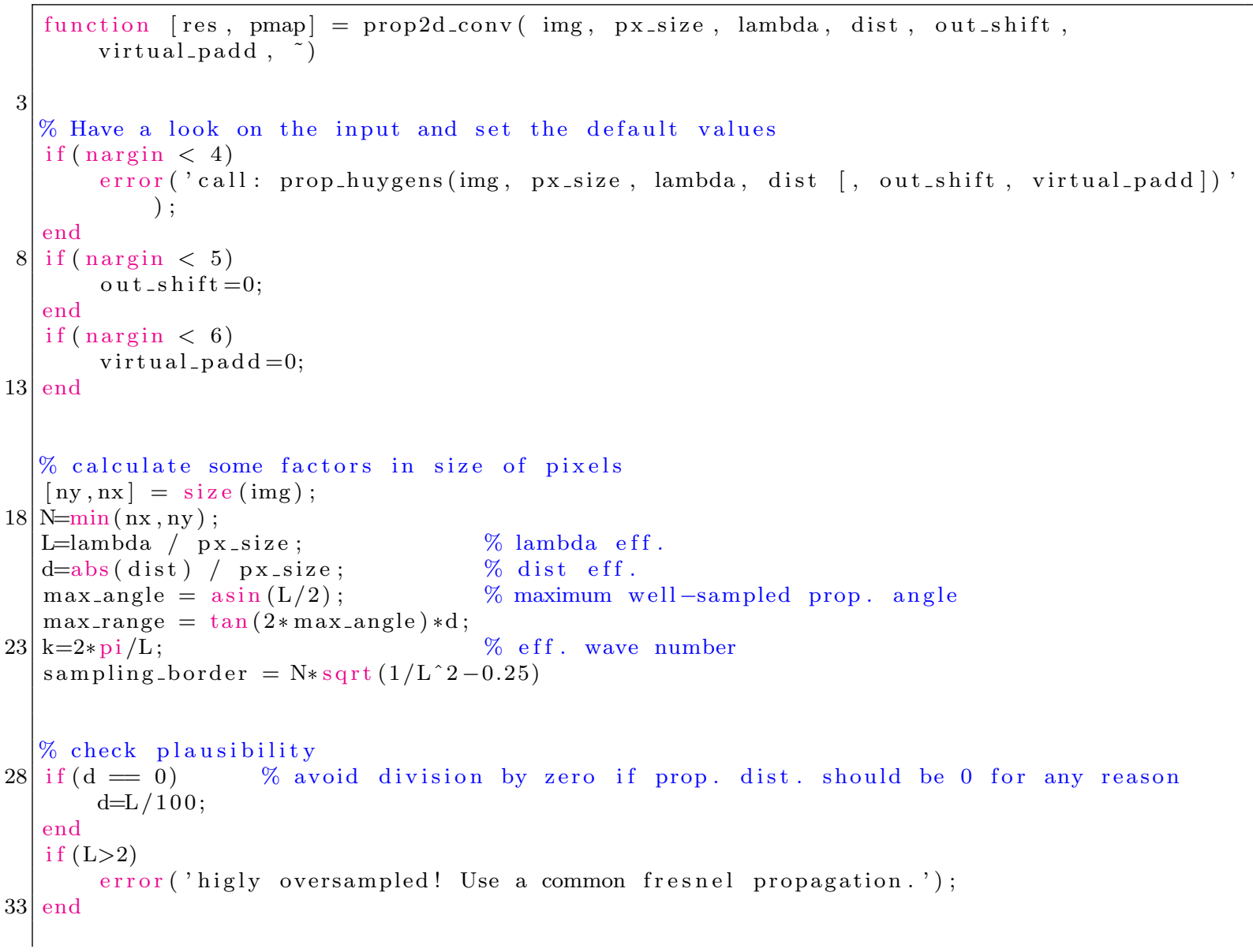




\section{Appendix}

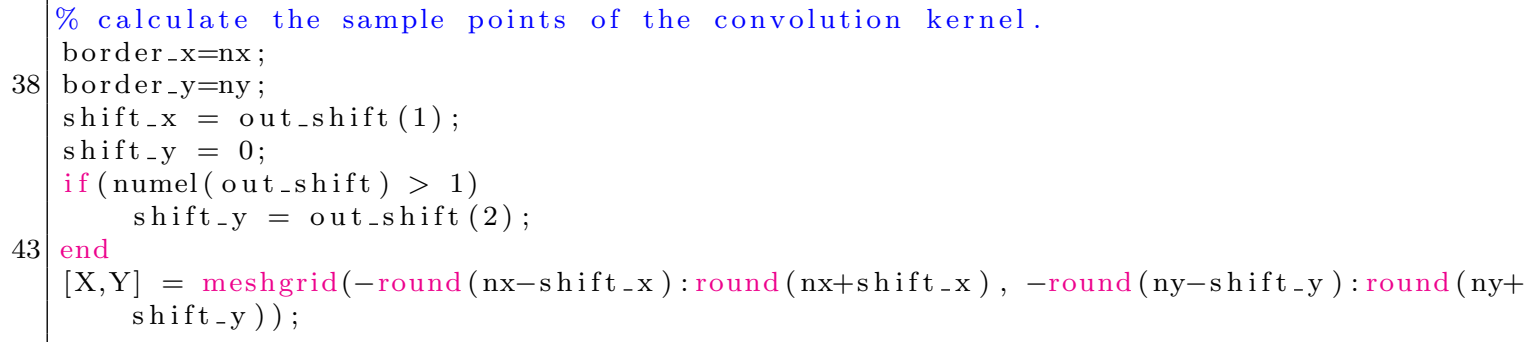

By default (no lateral shift), the convolution kernel is symmetrical around zero. It is crucial that the zero itself matches the centre pixel: For very short propagation distances, the kernel corresponds to a peak with an extension of one (or a few) pixels. For a correct result, this peak must not be interpolated to several pixels, as otherwise the propagated wave field is blurred. Therefore, the convolution kernel always has an odd number of pixels in each dimension. The outgoing spherical wave is calculated according to the Sommerfeld I solution (Eq. (4.20)) and limited by the "numerical obliquity factor" as follows:

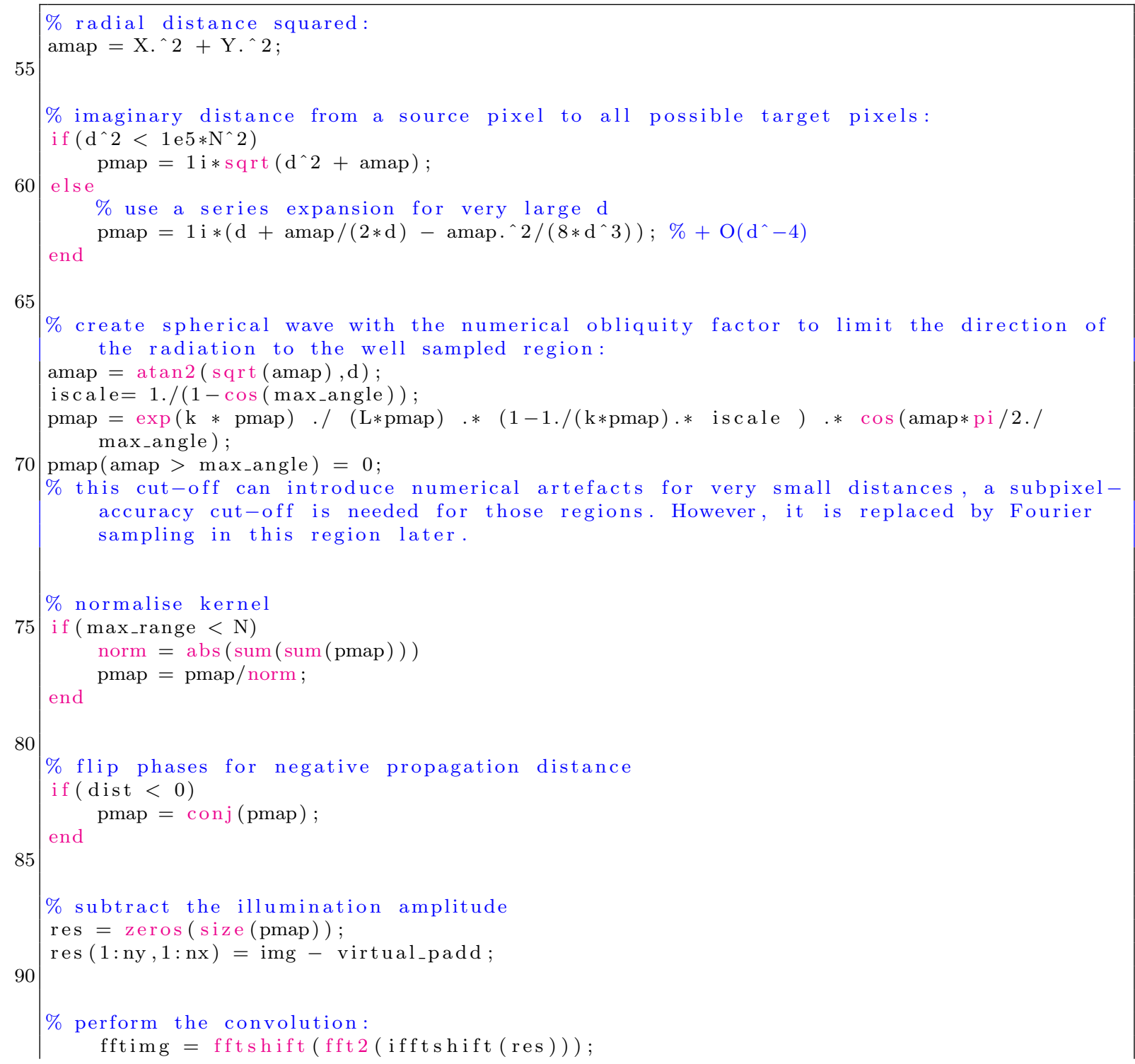




\subsection{Convolution-based numerical propagation}

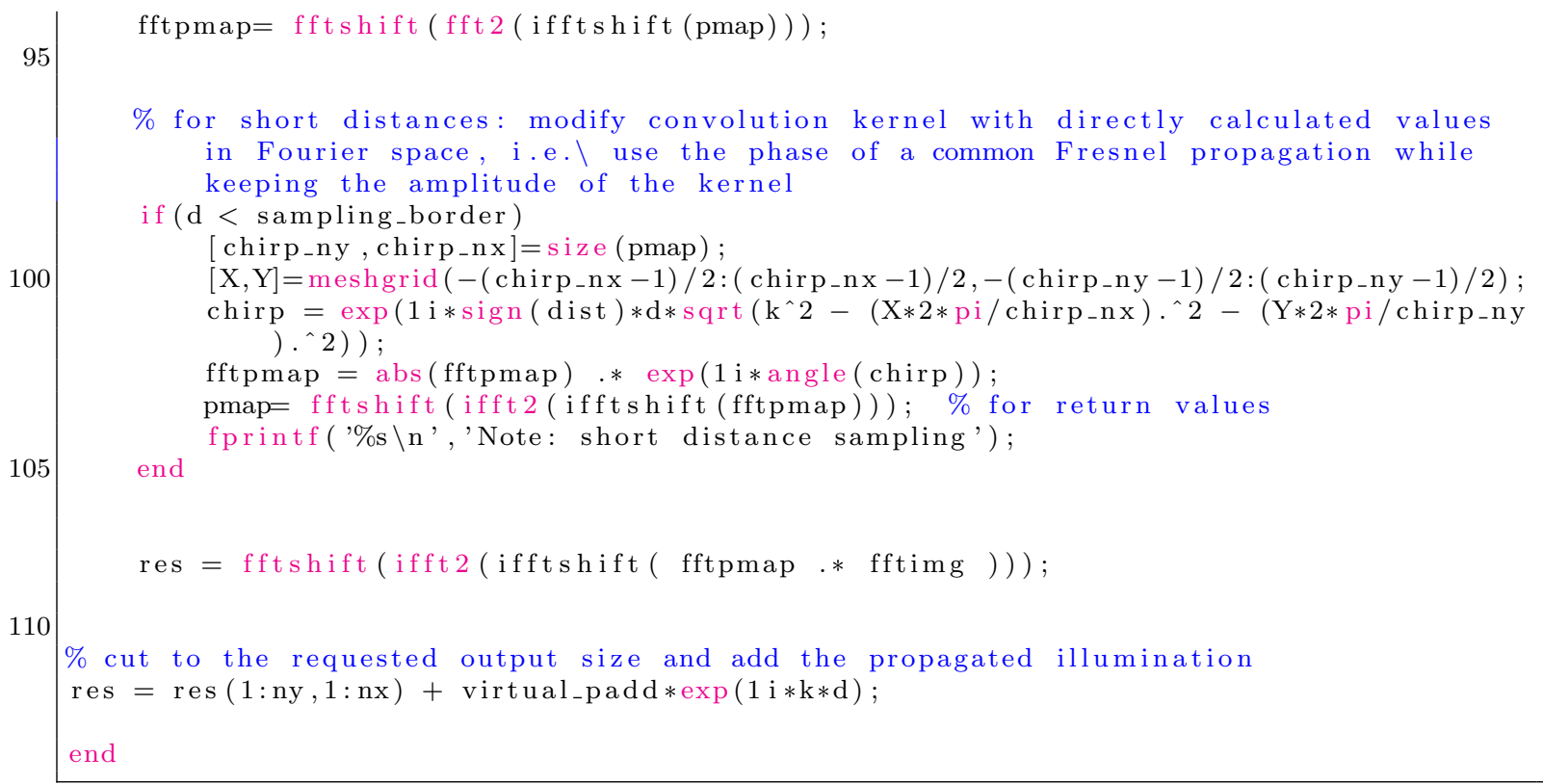

Code snippets for a qualitative comparison of the propagation methods in " $1+1 \mathrm{~d}$ " are listed in the following. First, a zone plate (see 9.3 for details) is created as an object and the output geometry is defined:

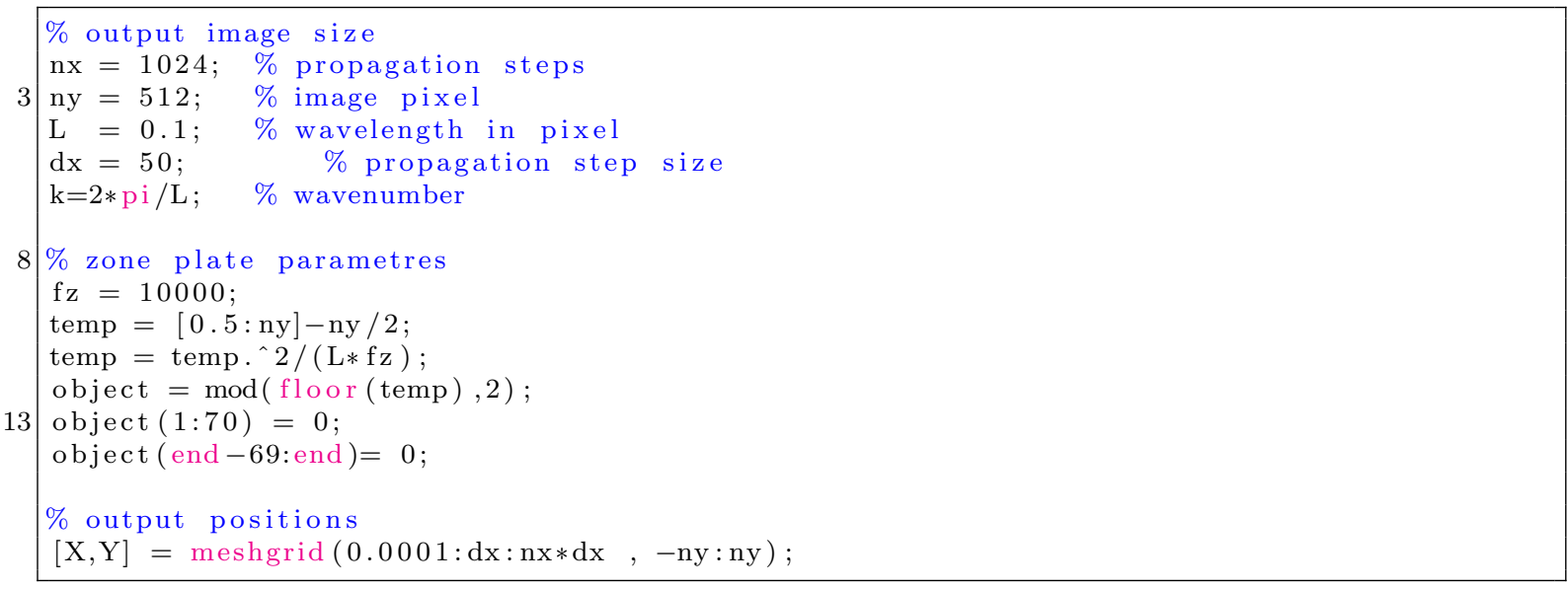

The classical convolution-based approach is then obtained by:

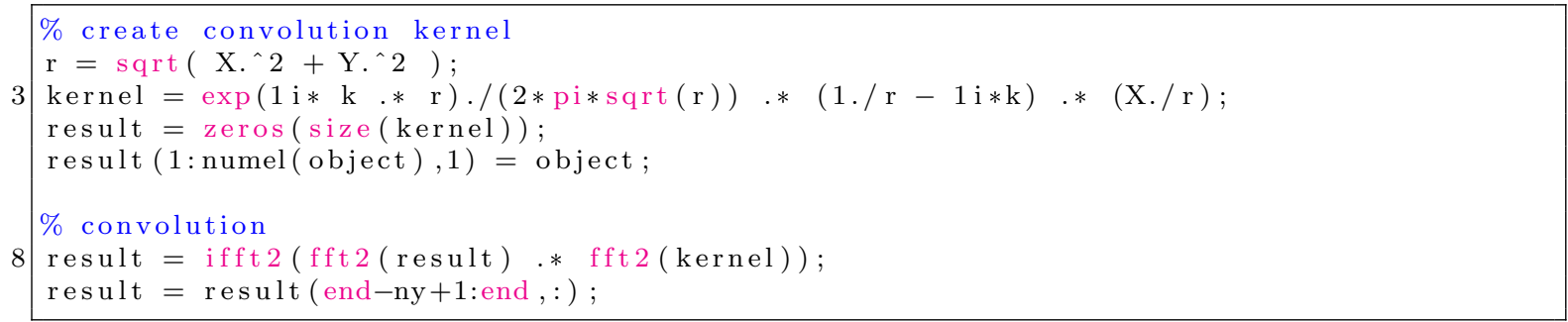

The Fresnel propagation can be achieved by:

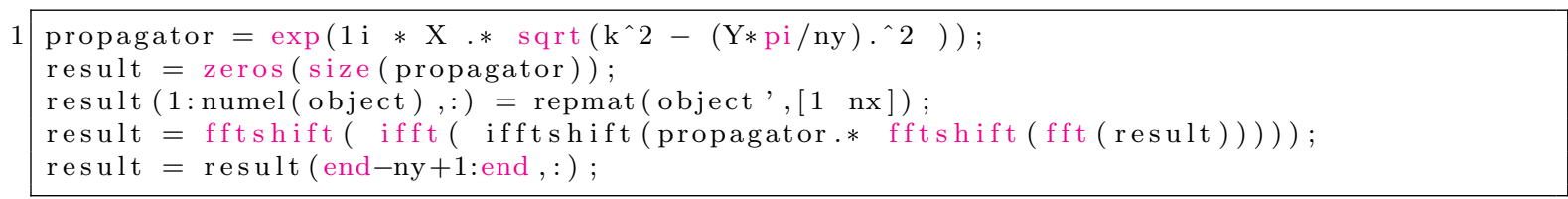

And the numerical obliquity factor propagation can be approximated as 


\section{Appendix}

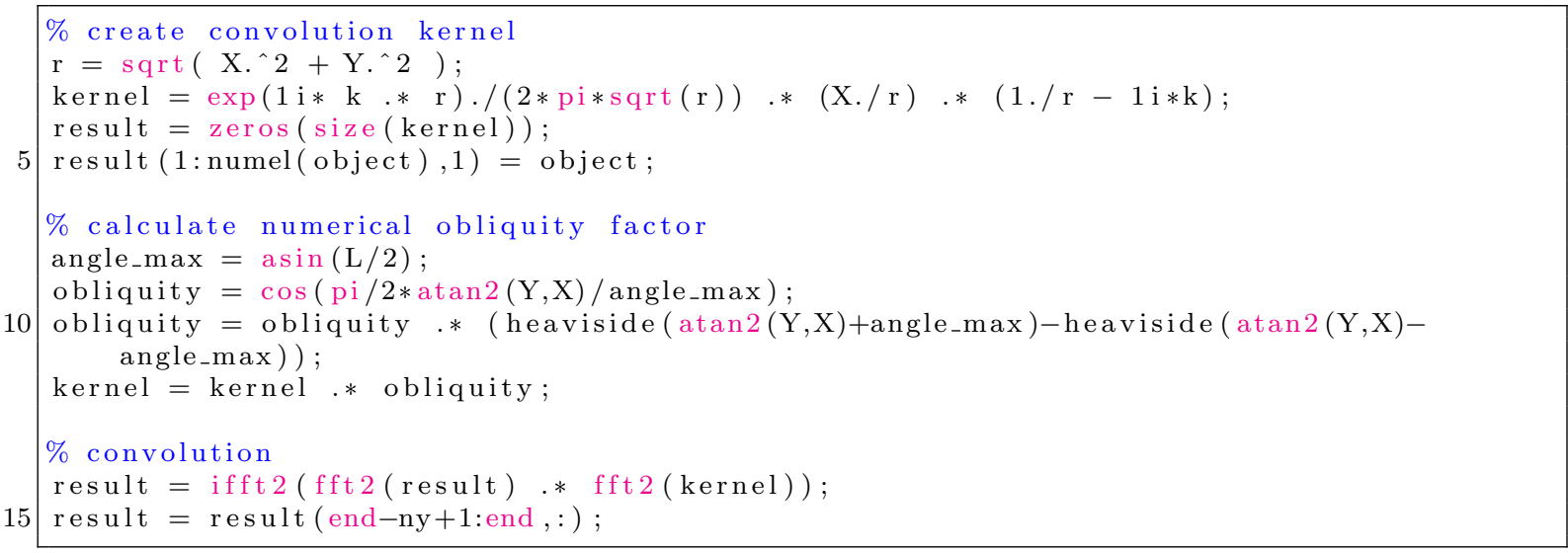

\subsection{Properties of binary zone plates}

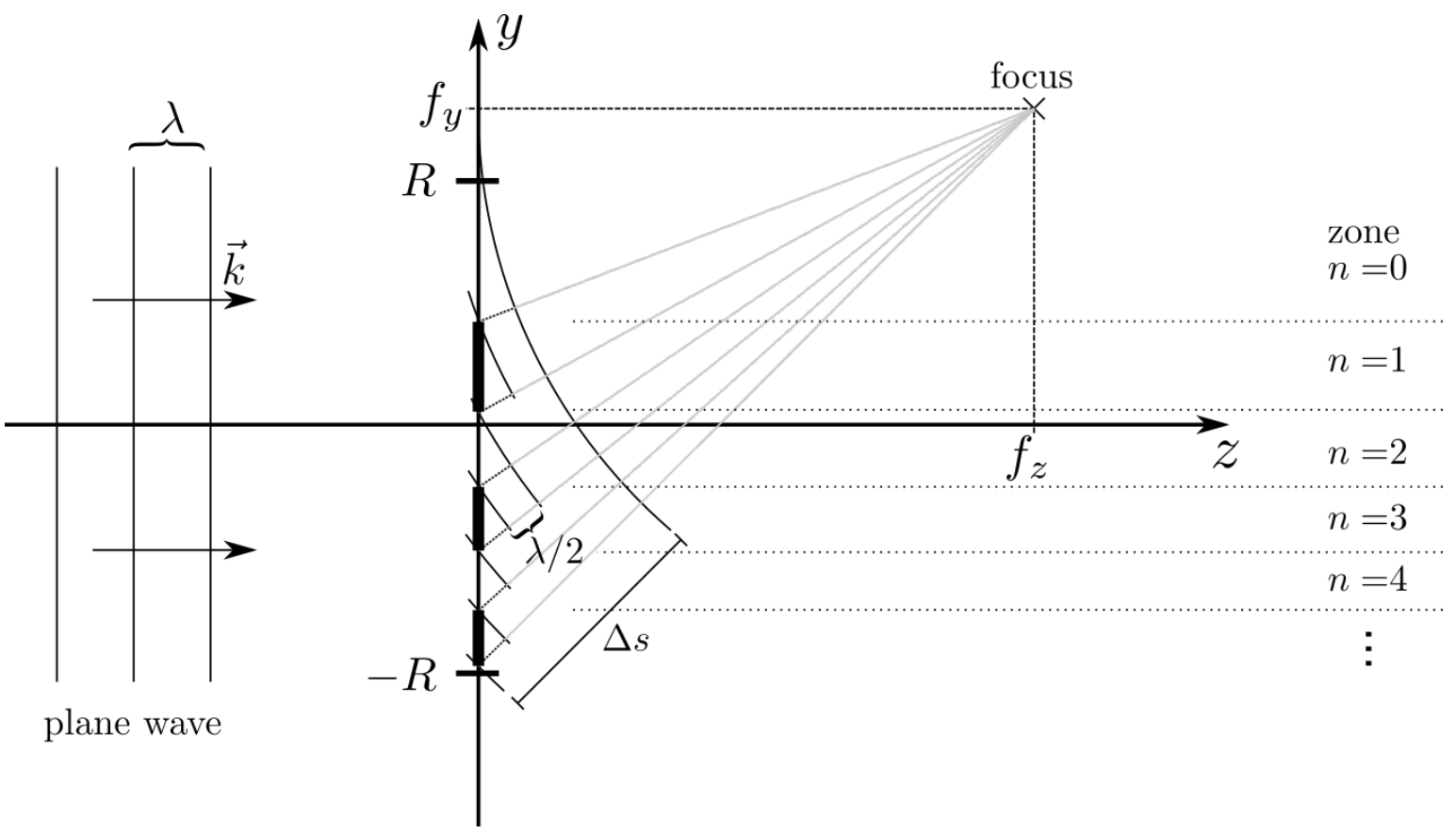

Figure 9.1: Schematic construction of a zone plate: The zones are defined by their distance to the focus. See text for details.

The inventor of the zone plate is said to be LORD RAYLEIGH, who describes the principle in a diary entry dated 11th April 1871: "The experiment of covering the odd Huygenian zones to amplify light in the middle was very successful" (see [134], footnote 18 p. 795). In Fig. 9.1 this principle is sketched for an incident plane wave arriving from the left, which is to be focused at the point $\vec{f}$. First of all, any reference point on the plate can be selected that allows the light beam to pass through to the focus without obstruction (here at $\left.\left(z=0, y=f_{y}\right)\right)$. Around this point, zones are defined 
which differ in their distance to the focus. The $n$-th zone consists of all points $(0, y)$ to which the realtion

$$
n \cdot \frac{\lambda}{2} \leq \Delta s<(n+1) \cdot \frac{\lambda}{2}
$$

applies. Whenever $y$ becomes so large that the difference in distance between $\Delta s$ and the reference path exceeds a new half-wavelength $\lambda / 2$, a new zone begins. Due to this definition, for each beam passing a zone there is a beam from the directly adjacent zones which is destructively interfering in the focus. In contrast, from each zone after the next zone a constructively interfering beam reaches the focus. Following Rayleigh's idea, every second zone can now be designed to absorb, so that only constructively interfering portions meet in the focus point and a maximum of intensity is obtained. Since the efficiency decreases due to absorbing areas, it is advisable not to cover the zones, but to produce them from a phase shifting material: If the phase in every second zone changes by $\pi$ (relative to the reference beam), the proportions of all zones interfere constructively in the focus. The difference in path length can be calculated using the

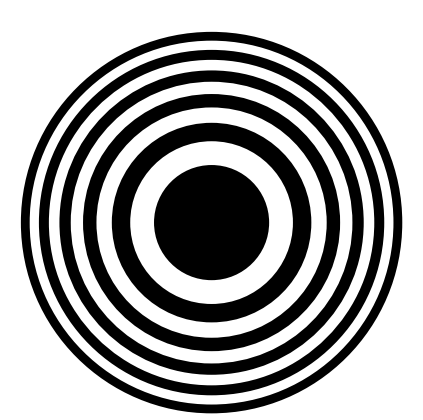

Figure 9.2: Zones of a symmetrical zone plate according to the ZP equation (??). Each transparent zone has the same area and contributes equally (in case of an infinitely thin zone plate) to the intensity of the focus.

definitions from Fig. 9.1 to

$$
\Delta s=\sqrt{f_{z}^{2}+\left(y-f_{y}\right)^{2}}-f_{z} \stackrel{!}{=} n \cdot \frac{\lambda}{2} .
$$

For given $f_{z}$ and $f_{y}$ one obtains

$$
n(y)=\frac{2}{\lambda}\left(\sqrt{f_{z}^{2}+\left(y-f_{y}\right)^{2}}-f_{z}\right)
$$

and

$$
y(n)=f_{y} \pm \sqrt{\left(\frac{n \lambda}{2}\right)^{2}+n \lambda f_{z}} .
$$

In Eq. (9.4) the quadratic term is usually neglected in very good approximation, since $\lambda$ for X-rays is very small in comparison to $|\vec{f}|$. For $f_{y}=0$ we obtain the Zone Plate equation

$$
y(n)^{2}=n \lambda f_{z}
$$


Figure 9.2 shows a two dimensional symmetrical zone plate calculated in this way. In addition to the desired focus at $f_{z}$, there are other foci in places where the distance difference (Eq. (9.2)) is an odd multiple of half the wavelength, i.e. $\Delta s=j \cdot n \cdot \frac{\lambda}{2}$ with $j=3,5,7, \ldots$ According to Eq. $(9.5)(n \rightarrow j \cdot n)$, the $n$-th order focus is located at

$$
f_{z, j}=\frac{f_{z, 1}}{j} .
$$

As with all diffraction optics there are also divergent parts of radiation, which seem to originate from a negative focus $j=-1,-3,-5 \ldots$. For $f_{y} \neq 0$, the focus is not on the optical axis and one speaks of an off-axis zone plate. In Fig. 9.1 it becomes obvious that it corresponds to a section of a normal zone plate. The characteristic zone-plateshaped wave field with highly divergent components and easily verifiable focal points is ideal for testing numerical propagation methods. 


\section{List of Figures}

2.1 Convolution and correlation . . . . . . . . . . . . . . . . . 16

2.2 Direct and circular convolution . . . . . . . . . . . . . . . . . . . 19

2.3 Principle of bilinear interpolation . . . . . . . . . . . . . . 20

2.4 Interpolation artefacts in Fourier space . . . . . . . . . . . . . . . 21

2.5 Fourier interpolation of a single mode . . . . . . . . . . . . . . . . 22

3.1 Phase-shift $\delta_{\omega}$ and absorption decrement $\beta_{\omega}$ for a model protein . . . . 27

3.2 Sketch of the projection approximation . . . . . . . . . . . . . 28

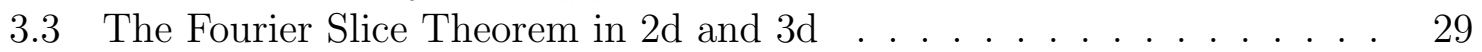

3.4 Phantom and corresponding Radon space in polar and Cartesian coordinates $($ sinogram $) \ldots \ldots . \ldots . . \ldots 31$

3.5 Projection of ellipse and square . . . . . . . . . . . . . . . . . . . 31

3.6 The Shepp and Logan "head phantom" . . . . . . . . . . . . . . . . . . 32

3.7 Discrete projection . . . . . . . . . . . . . . . . . . . . 33

3.8 Principle of backprojection . . . . . . . . . . . . . . . . . . 34

3.9 Backprojection for different numbers of projections . . . . . . . . 35

3.10 "Point spread function" of projection and direct backprojection . . . . 36

3.11 The principle of filtered backprojection . . . . . . . . . . . . . . 37

3.12 The Ram-Lak filter . . . . . . . . . . . . . . . . . . . . 38

3.13 First modes of a sinogram . . . . . . . . . . . . . . . . . . . . . . . . . . 40

3.14 IRR sinogram retrieval . . . . . . . . . . . . . . . . . . . . . 41

3.15 Comparison FBP vs. ART . . . . . . . . . . . . . . . . . . . 43

3.16 Principle of ART . . . . . . . . . . . . . . . . . . . 44

4.1 Integration Surface for Kirchhoffs Theorem . . . . . . . . . . . . . . . 49

4.2 Closing the integration surface at infinity . . . . . . . . . . . . . 49

4.3 Method of images . . . . . . . . . . . . . . . . 50

4.4 Angular spectrum of a wave field . . . . . . . . . . . . . . . 53

4.5 The chirp-function $\chi^{d} \ldots \ldots \ldots \ldots \ldots$. . . . . . . . . . . . . . . . . . . 54

$4.62 \mathrm{~d}$ propagation of a sectorised zone plate . . . . . . . . 56

4.7 Example of multislice propagation . . . . . . . . . . . . . . 57

4.8 Example of $1+1 \mathrm{~d}$ propagation by $2 \mathrm{~d}$ convolution . . . . . . . . . . 58

4.9 Comparison of propagation artefacts . . . . . . . . . . . 59

4.10 Near-Field-Aliasing . . . . . . . . . . . . . . . . . . . . 60

4.11 Artefacts from periodic repetition . . . . . . . . . . . . . 60

4.12 2d propagation with modified obliquity factor . . . . . . . . . . . 62

$4.131+1 \mathrm{~d}$ propagation with modified obliquity factor . . . . . . . . . . 63

4.14 Schematic of 3d propagation . . . . . . . . . . . . . . . 65

4.16 The Fresnel-Scaling-Theorem . . . . . . . . . . . . . . 67 
5.1 Imaging regimes in angular spectrum . . . . . . . . . . . . . . . 72

5.2 Intensity for different Fresnel numbers . . . . . . . . . . . . . . . . . . . . . . 74

5.3 The twin image . . . . . . . . . . . . . . . . . . 76

5.4 Shape of the contrast transfer function . . . . . . . . . . . . . 77

5.5 Lost spatial frequencies in PSD . . . . . . . . . . . . . . 78

5.6 Example of a holographic reconstruction . . . . . . . . . . . . . . 80

5.7 CTF reconstruction of a pure phase object . . . . . . . . . . . . . 83

5.8 Scheme of iterative phase retrieval . . . . . . . . . . . . . . 84

5.9 Example of iterative phase retrieval . . . . . . . . . . . . . 86

5.10 Typical setup for phase-contrast tomography . . . . . . . . . . . . . . . 87

5.11 IRP principle . . . . . . . . . . . . . . . . . . . . . . . 88

5.12 Consistency as a constraint . . . . . . . . . . . . . . . . 90

5.13 Consistency as a constraint with noise . . . . . . . . . . . . . . . 91

5.14 Comparison of $2 \mathrm{~d}$ and $3 \mathrm{~d}$ propagation based phase retrieval . . . . . . 94

5.15 IRP improvement on experimental data . . . . . . . . . . . . . . 95

5.16 Comparison of phase retrieval methods on experimental data . . . . . . 96

6.1 Motion artefacts of particles with constant velocity I . . . . . . . . . . 103

6.2 Motion artefacts of particles with constant velocity II . . . . . . . . . . 104

6.3 Two subsequent projections of a moving feature . . . . . . . . . . . 105

6.4 Direct reconstruction from a random walk . . . . . . . . . . . . 107

6.5 GINIX setup . . . . . . . . . . . . . . . . . . . 109

6.6 Artefacts of the flat-field correction . . . . . . . . . . . . . . 111

6.7 Waveguide types and cleaning effect . . . . . . . . . . . . . . . . . 112

6.8 Setup for fast tomography . . . . . . . . . . . . . . . . . . 115

6.9 Flat- and mean-field correction of detector images . . . . . . . . . . . 117

6.10 Alignment of a Sinogram . . . . . . . . . . . . . . . . 118

6.11 Alignment of a Linogram . . . . . . . . . . . . . . . . . . . . . . . . 118

6.12 CTF-based phase retrieval of a typical hologram . . . . . . . . . . . 119

6.13 3d reconstruction of the capillary . . . . . . . . . . . . . . 120

6.14 Localisation of particles in the volume . . . . . . . . . . . . . . . . . . . 122

6.15 Histogram of detected path length . . . . . . . . . . . . . . . . . . . . . . . . . . . . . . . 122

$6.16 N=1,10,100$ longest paths . . . . . . . . . . . . . . . 124

$6.17 N=10^{3}, 10^{4}$ longest paths . . . . . . . . . . . . . . . 125

6.18 Collective dynamics in the capillary . . . . . . . . . . . . . 126

6.19 Mean squared displacement of sedimenting spheres . . . . . . . . . . 127

6.20 Histogram of mean velocities of particles . . . . . . . . . . . . . 128

7.1 Artefacts from affine deformation and dissolving . . . . . . . . . . . 132

7.2 Local consistency in deforming objects . . . . . . . . . . . . . . . 133

7.3 Backprojection along dynamically curved paths . . . . . . . . . . . 134

7.4 Setup of the burning match experiment . . . . . . . . . . . . 136

7.5 Data treatment for $3 \mathrm{~d}$ reconstruction . . . . . . . . . . . . . . . 138

7.6 Estimated motion and improved reconstruction . . . . . . . . . . . . . 140

7.7 Rendered 3d structure of the burning match at different times. . . . . . 142

9.1 Schematic construction of a zone plate . . . . . . . . . . . . . . 152 
9.2 Zones of a symmetrical zone plate . . . . . . . . . . . . . . 153 



\section{Bibliography}

[1] Wilhelm Conrad Röntgen. Über eine neue art von strahlen. Sonderabbdruck aus den Sitzungsberichten der Würzburger Physik.-medic. Gesellschaft, pages 137147, Dec 1895.

[2] K. A. Nugent, T. E. Gureyev, D. F. Cookson, D. Paganin, and Z. Barnea. Quantitative Phase Imaging Using Hard X Rays. Physical Review Letters, 77(14):29612964, September 1996.

[3] David Paganin. Coherent X-ray optics. Oxford University Press, 2006.

[4] G. J. Williams, H. M. Quiney, B. B. Dhal, C. Q. Tran, K. A. Nugent, A. G. Peele, D. Paterson, and M. D. de Jonge. Fresnel Coherent Diffractive Imaging. Phys. Rev. Lett., 97(2):025506, 2006.

[5] Martin Dierolf, Andreas Menzel, Pierre Thibault, Philipp Schneider, Cameron M. Kewish, Roger Wepf, Oliver Bunk, and Franz Pfeiffer. Ptychographic X-ray computed tomography at the nanoscale. Nature, 467(7314):436-439, September 2010.

[6] F. Pfeiffer, O. Bunk, C. David, Martin Bech, G. Le Duc, A. Bravin, and P. Cloetens. High-resolution brain tumor visualization using three-dimensional x-ray phase contrast tomography. Physics in medicine and biology, 52(23):6923, 2007.

[7] S. W. Wilkins, T. E. Gureyev, D. Gao, A. Pogany, and A. W. Stevenson. Phasecontrast imaging using polychromatic hard X-rays. Nature, 384(6607):335-338, November 1996.

[8] P. Cloetens, W. Ludwig, J. Baruchel, D. Van Dyck, J. Van Landuyt, J. P. Guigay, and M. Schlenker. Holotomography: Quantitative phase tomography with micrometer resolution using hard synchrotron radiation x rays. Applied Physics Letters, 75(19):2912-2914, 1999.

[9] D. Paganin and K. A. Nugent. Noninterferometric Phase Imaging with Partially Coherent Light. Physical Review Letters, 80:2586-2589, Mar 1998.

[10] A. Ruhlandt, M. Krenkel, M. Bartels, and T. Salditt. Three-dimensional phase retrieval in propagation-based phase-contrast imaging. Physical Review A, 89:033847, 2014.

[11] Alexander Kostenko, K. Joost Batenburg, Andrew King, Erik Offerman, and Lucas J. van Vliet. Total variation minimization approach in in-line x-ray phasecontrast tomography. Optics Express, 21(10), may 2013. 
[12] Ilja N. Bronstein, Konstantin A. Semendjajew, Gerhard Musiol, and Heiner Mühlig. Taschenbuch der Mathematik. Wissenschaftlicher Verlag Harri Deutsch GmbH, 2008.

[13] John P. Lewis. Fast normalized cross-correlation. In Vision interface, volume 10, pages 120-123, 1995.

[14] William H. Press, Saul A. Teukolsky, William T. Vetterling, and Brian P. Flannery. Numerical Recipes: The Art of Schientific Computing. Cambridge University Press, 3 edition, 2007.

[15] Claude E. Shannon. Communication in the presence of noise. Proc. Institute of Radio Engineers, 37(1):10-21, jan. 1949.

[16] D. P. Petersen and D. Middleton. Sampling and reconstruction of wave-numberlimited functions in n-dimensional euclidean spaces. Information and Control, 5:279-323, 1962.

[17] Hyeokho Choi and David C. Munson. Direct-fourier reconstruction in tomography and synthetic aperture radar. International journal of imaging systems and technology, 9(1):1-13, 1998.

[18] Otto Glasser. Wilhelm Conrad Röntgen und die Geschichte der Röntgenstrahlen. Springer, 1995.

[19] Johann Radon. Über die bestimmung von funktionen durch ihre integralwerte längs gewisser mannigfaltigkeiten. Berichte über die Verhandlungen der Königlich Sächsischen Gesellschaft der Wissenschaften zu Leipzig. Mathematisch-Physische Klasse, 69:262-277, 1917.

[20] A. M. Cormack. Representation of a function by its line integrals, with some radiological applications. Journal of Applied Physics, 34(9):2722-2727, 1963.

[21] A. M. Cormack. Representation of a function by its line integrals, with some radiological applications. ii. Journal of Applied Physics, 35(10):2908-2013, Oct 1964.

[22] S. Webb. Historical experiments predating commercially available computed tomography. The British Journal of Radiology, 65(777):835-837, Sep. 1992.

[23] Vladimir Petrik, Vinothini Apok, Juliet A. Britton, B. Anthony Bell, and Marios C. Papadopoulos. Godfrey hounsfield and the dawn of computed tomography. Neurosurgery, 58(4):780-787, 2006.

[24] The Nobel Prize in Physiology or Medicine 1979. Nobel Media AB, 2014. Online resource; visited 2017-06-12.

[25] A. C. Kak and Malcolm Slaney. Principles of Computerized Tomographic Imaging. Society of Industrial and Applied Mathematics, 2001. 
[26] Frank Natterer. The Mathematics of Computerized Tomography. Wiley, New York, 1986.

[27] Thorsten M. Buzug. Computed Tomography: From Photon Statistics to Modern Cone-Beam CT. Springer, 2008. ISBN: 978-3-642-07257-4.

[28] Max Born and Emil Wolf. Principles of optics: electromagnetic theory of propagation, interference and diffraction of light. Elsevier, 1980.

[29] Joseph W Goodman. Introduction to Fourier optics. Roberts and Company Publishers, 2005.

[30] Jens Als-Nielsen and Des McMorrow. Elements of Moder X-ray Physics. John Wiley \& Sons, 2 edition, 2011.

[31] P. Thibault, V. Elser, C. Jacobsen, D. Shapiro, and D. Sayre. Reconstruction of a yeast cell from x-ray diffraction data. Acta Crystallographica A, 62(4), 2006.

[32] B.L. Henke, E.M. Gullikson, and J.C. Davis. X-ray interactions: photoabsorption, scattering, transmission, and reflection at $\mathrm{e}=50-30000 \mathrm{ev}, \mathrm{z}=1-92$. Atomic Data and Nuclear Data Tables, 54(2):181-342, July 1993.

[33] Jianwei Miao, Friedrich Förster, and Ofer Levi. Equally sloped tomography with oversampling reconstruction. Physical Review B, 72:52103, 2005.

[34] Daniël M. Pelt and Kees Joost Batenburg. Improving filtered backprojection reconstruction by data-dependent filtering. IEEE Transactions on Image Processing, 23(11):4750 - 4762, Jul 2014.

[35] Sigurdur Helgason. The radon transform on euclidean spaces, compact two-point homogeneous spaces, and grassmann manifolds. Acta Mathematica, 113(1):153, 1965 .

[36] Donald Ludwig. The radon transform on euclidean space. Communications on Pure and Applied Mathematics, 19(1):49-81, 1966.

[37] Hiroyuki Kudo and Tsuneo Saito. Sinogram recovery with the method of convex projections for limited-data reconstruction in computed tomography. J. Opt. Soc. Am. A, 8(7):1148-1160, July 1991.

[38] Menahem Nassi, William R. Brody, Barry P. Medoff, and Albert Macovski. Iterative reconstruction-reprojection: An algorithm for limited data cardiac-computed tomography. IEEE Transactions on Biomedical Engineering, BME-29(5):333341, Mai 1982.

[39] Godfrey Newbold Hounsfield. Method of an apparatus for examining a body by radiation such as $\mathrm{x}$ or gamma radiation, 1975.

[40] Stefan Kaczmarz. Angenäherte auflösung von systemen linearer gleichungen. Bulletin International de l'Academie Polonaise des Sciences et des Lettres, 35:355357, 1937. 
[41] G. T. Herman. Fundamentals of computerized tomography: Image reconstruction from projection. Springer, 2009.

[42] Steven H. Schot. Eighty years of sommerfeld's radiation condition. Historia Mathematica, 19:385-401, 1992.

[43] H. A. Bethe. Theory of diffraction by small holes. Physical Review, 66(7-8):163182, Oct 1944.

[44] Arnold Sommerfeld. Mathematische theorie der diffraction. Mathematische Annalen, 47:317-374, 1896.

[45] David G. Voelz and Michael C. Roggemann. Digital simulation of scalar optical diffraction: revisiting chirp function sampling criteria and consequences. Applied Optics, 48(32):6132-6142, Nov. 2009.

[46] Fabin Shen and Anbo Wang. Fast-fourier-transform based numerical integration method for the rayleigh-sommerfeld diffraction formula. Applied Optics, 45(6):1102-1110, Feb. 2006.

[47] Kenan Li, Michael Wojcik, and Chris Jacobsen. Multislice does it all: calculating the performance of nanofocusing x-ray optics. Optics Express, 25(3):1831-1846, Feb. 2017.

[48] Kyoji Matsushima and Tomoyoshi Shimobaba. Band-limited angular spectrum method for numerical simulation of free-space propagation in far and near fields. Optics Express, 17(22):19662-19673, Oct. 2009.

[49] Aike Ruhlandt and Tim Salditt. Three-dimensional propagation in near-field tomographic x-ray phase retrieval. Acta Crystallographica A, 72:215-221, 2016.

[50] Pierre Thibault, Martin Dierolf, Andreas Menzel, Oliver Bunk, Christian David, and Franz Pfeiffer. High-Resolution Scanning X-ray Diffraction Microscopy. Science, 321(5887):379-382, 2008.

[51] Timm Weitkamp, Ana Diaz, Christian David, Franz Pfeiffer, Marco Stampanoni, Peter Cloetens, and Eric Ziegler. X-ray phase imaging with a grating interferometer. Optics Express, 13(16):6296-6304, Aug 2005.

[52] Peter RT Munro, Konstantin Ignatyev, Robert D Speller, and Alessandro Olivo. Phase and absorption retrieval using incoherent x-ray sources. Proceedings of the National Academy of Sciences, 109(35):13922-13927, 2012.

[53] CK Hagen, PRT Munro, M Endrizzi, PC Diemoz, and A Olivo. Low-dose phase contrast tomography with conventional x-ray sources. Medical physics, 41(7):070701, 2014.

[54] I Zanette, Tunhe Zhou, Anna Burvall, Ulf Lundström, Daniel H Larsson, M Zdora, P Thibault, F Pfeiffer, and Hans M Hertz. Speckle-based x-ray phasecontrast and dark-field imaging with a laboratory source. Physical Review Letters, 112(25):253903, 2014. 
[55] S. Lagomarsino, A. Cedola, P. Cloetens, S. Di Fonzo, W. Jark, G. Soullie, and C. Riekel. Phase contrast hard x-ray microscopy with submicron resolution. Applied Physics Letters, 71(18):2557-2559, 1997.

[56] Marco Stampanoni, Rajmund Mokso, Federica Marone, Joan Vila-Comamala, Sergey Gorelick, Pavel Trtik, Konstantin Jefimovs, and Christian David. Phasecontrast tomography at the nanoscale using hard $\mathrm{x}$ rays. Physical Review B, 81(14):140105, April 2010.

[57] Dennis Gabor. A new microscopic principle. Nature (London), 161:777-778, 1948.

[58] M. Bartels, M. Krenkel, J. Haber, R. N. Wilke, and T. Salditt. X-ray holographic imaging of hydrated biological cells in solution. Physical Review Letters, 114:048103, Jan 2015.

[59] Johannes Hagemann and Tim Salditt. The fluence-resolution relationship in holographic and coherent diffractive imaging. Journal of Applied Crystallography, 50:531-538, 2017.

[60] Nikolay I. Zheludev. What diffraction limit? Nature materials, 7(6):420-422, 2008.

[61] H. Toyokawa, Ch. Broennimann, E.F. Eikenberry, B. Henrich, M. Kawase, M. Kobas, P. Kraft, M. Sato, B. Schmitt, M. Suzuki, H. Tanida, and T. Uruga. Single photon counting pixel detectors for synchrotron radiation experiments. Nucl. Instrum. Methods Phys. Res., Sect. A, 623(1):204-206, November 2010.

[62] J. P. Guigay. Fourier transform analysis of fresnel diffraction patterns and in-line holograms. Optik, 49(1):121-125, 1977.

[63] A. Pogany, D. Gao, and S. W. Wilkins. Contrast and resolution in imaging with a microfocus x-ray source. Review of Scientific Instruments, 68(7):2774-2782, 1997.

[64] L. Turner, B. Dhal, J. Hayes, A. Mancuso, K. Nugent, D. Paterson, R. Scholten, C. Tran, and A. Peele. X-ray phase imaging: Demonstration of extended conditions for homogeneous objects. Optics Express, 12(13):2960-2965, June 2004.

[65] Timur E. Gureyev, Timothy J. Davis, Andrew Pogany, Sheridan C. Mayo, and Stephen W. Wilkins. Optical phase retrieval by use of first born- and rytov-type approximations. Applied Optics, 43(12):2418-2430, April 2004.

[66] Max Langer, Peter Cloetens, Alexandra Pacureanu, and Françoise Peyrin. X-ray in-line phase tomography of multimaterial objects. Optics Letters, 37(11):21512153, June 2012.

[67] Julian Moosmann, Ralf Hofmann, and Tilo Baumbach. Single-distance phase retrieval at large phase shifts. Opt. Express, 19(13):12066-12073, June 2011. 
[68] P. Cloetens. Contribution to Phase Contrast Imaging, Reconstruction and Tomography with Hard Synchrotron Radiation. PhD thesis, Vrije Universiteit Brussel, 1999.

[69] L.J. Allen and M.P. Oxley. Phase retrieval from series of images obtained by defocus variation. Optics Communications, 199:65-75, 2001.

[70] Hong You-Li, Zhang Kai, Wang Zhi-Li, Zhu Zhong-Zhu, Zhao Xue-Jiao, Huang Wan-Xia, Yuan Qing-Xi, Zhu Pei-Ping, and WAllen Zi-Yu. Reconstructing a complex field from a series of its near-field diffraction patterns. Chinese Physics B, 21(10):104202, 2012.

[71] M. Krenkel, M. Bartels, and T. Salditt. Transport of intensity phase reconstruction to solve the twin image problem in holographic x-ray imaging. Optics Express, 21(2):2220-2235, January 2013.

[72] S Zabler, P Cloetens, J-P Guigay, J Baruchel, and M Schlenker. Optimization of phase contrast imaging using hard x rays. Review of Scientific Instruments, 76(7):073705, 2005.

[73] Martin Krenkel, Mareike Toepperwien, Frauke Alves, and Tim Salditt. Threedimensional single-cell imaging with X-ray waveguides in the holographic regime. Acta Crystallogr. A, 73(4):282-292, July 2017.

[74] A. Groso, R. Abela, and M. Stampanoni. Implementation of a fast method for high resolution phase contrast tomography. Opt. Express, 14(18):8103-8110, September 2006.

[75] D. Paganin, S. C. Mayo, T. E. Gureyev, P. R. Miller, and S. W. Wilkins. Simultaneous phase and amplitude extraction from a single defocused image of a homogeneous object. Journal of Microscopy, 206(Pt 1):33-40, 2002.

[76] Yoni De Witte, Matthieu Boone, Jelle Vlassenbroeck, Manuel Dierick, and Luc Van Hoorebeke. Bronnikov-aided correction for x-ray computed tomography. Journal of the Optical Society of America A, Optics and Image Science, 26(4):890-894, April 2009.

[77] Jonathan Nemirovsky and Efrat Shimron. Utilizing bochners theorem for constrained evaluation of missing fourier data. arXiv preprint arXiv:1506.03300 [physics.med-ph], 2015.

[78] Simon Maretzke. A uniqueness result for propagation-based phase contrast imaging from a single measurement. Inverse Problems, 31:065003, May 2015.

[79] R. W. Gerchberg and W. O. Saxton. A Practical Algorithm for the Determination of Phase from Image and Diffraction Plane Pictures. Optik, 35(2):237-246, November 1972.

[80] J. R. Fienup. Phase retrieval algorithms: a comparison. Applied Optics, 21(15):2758-2769, Aug. 1982. 
[81] D: Russell Luke. Relaxed averaged alternating reflections for diffraction imaging. Inverse Problems, 21(1):37-50, 2005.

[82] Andrei V. Bronnikov. Theory of quantitative phase-contrast computed tomography. J. Opt. Soc. Am. A, 19(3):472-480, March 2002.

[83] T. E. Gureyev, D. M. Paganin, G. R. Myers, Y. I. Nesterets, and S. W. Wilkins. Phase-and-amplitude computer tomography. Applied Physics Letters, 89(3):034102, July 2006.

[84] R. Mokso, P. Cloetens, E. Maire, W. Ludwig, and J.Y. Buffiere. Nanoscale zoom tomography with hard x rays using Kirkpatrick-Baez optics. Appl. Phys. Lett., 90:144104, 2007.

[85] M. Krenkel, A. Markus, M. Bartels, C. Dullin, F. Alves, and T. Salditt. Phasecontrast zoom tomography reveals precise locations of macrophages in mouse lungs. Scientific Reports, 5:09973, 2015.

[86] M. Töpperwien, M. Krenkel, D. Vincenz, F. Stöber, A. M. Oelschlegel, J. Goldschmidt, and T. Salditt. Three-dimensional mouse brain cytoarchitecture revealed by laboratory-based x-ray phase-contrast tomography. Scientific Reports, $7: 42847,2017$.

[87] A. Groso, M. Stampanoni, R. Abela, P. Schneider, S. Linga, and R. Müller. Phase contrast tomography: An alternative approach. Applied Physics Letters, 88:214104, 2006.

[88] Charles L Byrne. Applied iterative methods. AK Peters Wellesley, 2008.

[89] J. Frank and P. Penczek. On the correction of the contrast transfer function in biological electron microscopy. Optik, 98(3):125, 1995.

[90] Martin Krenkel. Cone-beam x-ray phase-contrast tomography for the observation of single cells in whole organs. PhD thesis, Universitäet Göettingen, 2015.

[91] Matthias Bartels, Marius Priebe, Robin N. Wilke, Sven Krüger, Klaus Giewekemeyer, Sebastian Kalbfleisch, Christian Olendrowitz, C, Michael Sprung, and Tim Salditt. Low-dose three-dimensional hard x-ray imaging of bacterial cells. Opt. Nanoscopy, 1(1):10, 2012.

[92] K. Giewekemeyer, S. P. Krüger, S. Kalbfleisch, M. Bartels, C. Beta, and T. Salditt. X-ray propagation microscopy of biological cells using waveguides as a quasipoint source. Phys. Rev. A, 83(2):023804, February 2011.

[93] H Nicolai, B Herzhaft, EJ Hinch, L Oger, and E Guazzelli. Particle velocity fluctuations and hydrodynamic self-diffusion of sedimenting non-brownian spheres. Physics of Fluids, 7(1):12-23, Jan 1995.

[94] Jose R Guzman-Sepulveda, Samiul Amin, E Neil Lewis, and Aristide Dogariu. Full characterization of colloidal dynamics at low péclet numbers. Langmuir, 31(38):10351-10357, 2015. 
[95] Adnan Hamid, Molina John J, and Ryoichi Yamamoto. Sedimentation of nonbrownian spheres at high volume fractions. Soft Matter, 9(42):10056-10068, 2013.

[96] Roberto Piazza, Stefano Buzzaccaro, and Eleonora Secchi. The unbearable heaviness of colloids: facts, surprises, and puzzles in sedimentation. Journal of Physics: Condensed Matter, 24(28):284109, 2012.

[97] P. N. Segrè, E. Herbolzheimer, and P. M. Chaikin. Long-range correlations in sedimentation. Physical Review Letters, 79(13):2574-2577, 1997.

[98] Johannes Möller and Theyencheri Narayanan. Velocity fluctuations in sedimenting brownian particlesmöller, johannes and narayanan, theyencheri. Physical Review Letters, 118(19):198001, May 2017.

[99] Philip N Segrè, Fang Liu, P Umbanhowar, and David A Weitz. An effective gravitational temperature for sedimentation. Nature, 409(6820):594-597, 2001.

[100] Russel E. Caflisch and Jonathan H. C. Luke. Variance in the sedimentation speed of a suspension. The Physics of Fluids, 28(3):759-760, 1985.

[101] J. M. Ham and G. M. Homsy. Hindered settling and hydrodynamic dispersion in quiescent sedimenting suspensions. International Journal of Multiphase Flow, 14(5):533-546, 1988.

[102] Manuel Guizar-Sicairos, Jaap J Boon, Kevin Mader, Ana Diaz, Andreas Menzel, and Oliver Bunk. Quantitative interior x-ray nanotomography by a hybrid imaging technique. Optica, 2(3):259-266, 2015.

[103] Tristan van Leeuwen, Simon Maretzke, and K. Joost Batenburg. Automatic alignment for three-dimensional tomographic reconstruction. arXiv preprint arXiv:1705.08678 [math.NA], 2017.

[104] Martin Uecker, Shuo Zhang, and Jens Frahm. Nonlinear inverse reconstruction for real-time mri of the human heart using undersampled radial flash. Magnetic resonance in medicine, 63(6):1456-1462, 2010.

[105] S. Bonnet, A. Koenig, S. Roux, P. Hugonnard, R. Guillemaud, and P. Grangeat. Dynamic x-ray computed tomography. Proceedings of the IEEE, 91(10):15741587, Oct 2003.

[106] Marc Kachelriess and Willi A. Kalender. Electrocardiogram-correlated image reconstruction from subsecond spiral computed tomography scans of the heart. Medical Physics, 25(12):2417-2431, 1998.

[107] Thomas Flohr and Bernd Ohnesorge. Heart rate adaptive optimization of spatial and temporal resolution for electrocardiogram-gated multislice spiral ct of the heart. Journal of Computer Assisted Tomography, 25(6):907-923, 2001.

[108] Daniel A. Schwyn, Rajmund Mokso, Simon M. Walker, Michael Doube, Martina Wicklein, Graham K. Taylor, Marco Stampanoni, and Holger G. Krapp. Highspeed x-ray imaging on the fly. Synchrotron Radiation News, 26(2):4-10, 2013. 
[109] Simon M Walker, Daniel A Schwyn, Rajmund Mokso, Martina Wicklein, Tonya Müller, Michael Doube, Marco Stampanoni, Holger G Krapp, and Graham K Taylor. In vivo time-resolved microtomography reveals the mechanics of the blowfly flight motor. PLoS Biology, 12(3):e1001823, 2014.

[110] Sebastian Kalbfleisch. A dedicated endstation for waveguide-based x-ray imaging. $\mathrm{PhD}$ thesis, Universität Göttingen, 2012.

[111] Tim Salditt, Markus Osterhoff, Martin Krenkel, Robin N. Wilke, Marius Priebe, Matthias Bartels, Sebastian Kalbfleisch, and Michael Sprung. Compound focusing mirror and x-ray waveguide optics for coherent imaging and nano-diffraction. Journal of Synchrotron Radiation, 2015.

[112] X-ray sCMOS camera C12849 series data sheet. Hamamatsu Photonics K.K., 2015.

[113] J. Hagemann, A.-L. Robisch, D. R. Luke, C. Homann, T. Hohage, P. Cloetens, H. Suhonen, and T. Salditt. Reconstruction of wave front and object for inline holography from a set of detection planes. Optics Express, 22(10):11552-11569, may 2014.

[114] H. Neubauer, S. Hoffmann, M. Kanbach, J. Haber, S. Kalbfleisch, S. P. Krüger, and T. Salditt. High aspect ratio x-ray waveguide channels fabricated by e-beam lithography and wafer bonding. Journal of Applied Physics, 115(21):214305, 2014.

[115] H.-Y. Chen, S. Hoffmann, and T. Salditt. X-ray beam compression by tapered waveguides. Applied Physics Letters, 106(19):194105, 2015.

[116] S. Hoffmann-Urlaub, P. Höhne, M. Kanbach, and T. Salditt. Advances in fabrication of x-ray waveguides. Microelectronic Engineering, 164:135-138, 2016.

[117] A. Ruhlandt, M. Töpperwien, M. Krenkel, R. Mokso, and T. Salditt. Four dimensional material movies: High speed phase-contrast tomography by backprojection along dynamically curved paths. Scientific Reports, 7, July 2017.

[118] K. Aditya Mohan, S. V. Venkatakrishnan, John W. Gibbs, Emine Begum Gulsoy, Xianghui Xiao, Marc De Graef, Peter W. Voorhees, and Charles A. Bouman. Timbir: A method for time-space reconstruction from interlaced views. IEEE Transactions on Computational Imaging, 1(2):96-111, 2015.

[119] JW Gibbs, K Aditya Mohan, EB Gulsoy, AJ Shahani, X Xiao, CA Bouman, M De Graef, and PW Voorhees. The three-dimensional morphology of growing dendrites. Scientific reports, 5, 2015.

[120] Daniila Kazantsev, Enyua Guo, Anders Kaestner, William R. B. Lionheart, Julian Bent, Philip J. Withers, and Peter D. Lee. Temporal sparsity exploiting nonlocal regularization for $4 \mathrm{~d}$ computed tomography reconstruction. Journal of X-Ray Science and Technology, 24(2):207-2019, 2016. 
[121] Sébastien Roux, Laurent Desbat, Anne Koenig, and Pierre Grangeat. Exact reconstruction in 2d dynamic ct: compensation of time-dependent affine deformations. Physics in medicine and biology, 49(11):2169, 2004.

[122] A. Katsevich. An accurete approximative algorithm for motion compensation in two-dimensional tomography. Inverse Problems, 26(3):065007, 2010.

[123] BN Hahn. Efficient algorithms for linear dynamic inverse problems with known motion. Inverse Problems, 30(3):035008, 2014.

[124] Christophe Blondel, Régis Vaillant, Grégoire Malandain, and Nicholas Ayache. 3d tomographic reconstruction of coronary arteries using a precomputed 4d motion field. Physics in Medicine and Biology, 49(11):2197, 2004.

[125] Christophe Blondel, Grégoire Malandain, Régis Vaillant, and Nicholas Ayache. Reconstruction of coronary arteries from a single rotational x-ray projection sequence. IEEE Transactions on Medical Imaging, 25(5):653-663, 2006.

[126] Dirk Schafer, Jörn Borgert, Volker Rasche, and Michael Grass. Motioncompensated and gated cone beam filtered back-projection for 3-d rotational x-ray angiography. IEEE transactions on medical imaging, 25(7):898-906, 2006.

[127] Simon Rit, David Sarrut, and Laurent Desbat. Comparison of analytic and algebraic methods for motion-compensated cone-beam ct reconstruction of the thorax. IEEE transactions on medical imaging, 28(10):1513-1525, 2009.

[128] Tianfang Li, Albert Koong, and Lei Xing. Enhanced 4d cone-beam ct with inter-phase motion model. Medical Physics, 34(9):3688-3695, 2007.

[129] Atsushi Momose, Wataru Yashiro, Sébastien Harasse, and Hiroaki Kuwabara. Four-dimensional x-ray phase tomography with talbot interferometry and white synchrotron radiation: dynamic observation of a living worm. Optics Express, 19(9):8423-8432, 2011.

[130] M. Stampanoni, F. Marone, P. Modregger, B. Pinzer, T. Thüring, J. VilaComamala, C. David, and R. Mokso. Tomographic hard x-ray phase contrast micro- and nano-imaging at tomcat. AIP Conference Proceedings, 1266(1):13$17,2010$.

[131] R Mokso, F Marone, S Irvine, M Nyvlt, D Schwyn, K Mader, G K Taylor, H G Krapp, M Skeren, and M Stampanoni. Advantages of phase retrieval for fast x-ray tomographic microscopy. Journal of Physics D: Applied Physics, 46(49):494004, 2013.

[132] Julie L. Fife, Michel Rappaz, Mattia Pistone, Tine Celcer, Gordan Mikuljan, and Marco Stampanoni. Development of a laser-based heating system for in situ synchrotron-based X-ray tomographic microscopy. Journal of Synchrotron Radiation, 19(3):352-358, May 2012. 
[133] C. Liu. Beyond Pixels: Exploring New Representations and Applications for Motion Analysis. PhD thesis, Massachusetts Institute of Technology, 2009.

[134] Eugene Hecht. Optik. Oldenbourg Wissenschaftsverlag, 8 edition, 2009. 


\subsection{Own publications}

1. A. Ruhlandt, M. Töpperwien, M. Krenkel, R. Mokso, and T. Salditt

Four dimensional material movies: High speed phase-contrast tomography by backprojection along dynamically curved paths

Scientific Reports 7: p. 6487 (2017)

2. A. Ruhlandt and T. Salditt

Three-dimensional propagation in near-field tomographic X-ray phase retrieval Acta Crystallographica A 72: p. 1-7 (2016)

3. M. Vassholz, B. Koberstein-Schwarz, A. Ruhlandt, M. Krenkel, T. Salditt New X-Ray Tomography Method Based on the 3D Radon Transform Compatible with Anisotropic Sources

Physical Review Letters 116: p. 088101 (2016)

4. A. Ruhlandt, M. Krenkel, M. Bartels, and T. Salditt

Three-dimensional phase retrieval in propagation-based phase-contrast imaging

Physical Review A 89: p. 033847 (2014)

5. O. Schulz, C. Pieper, M. Clever, J. Pfaff, A. Ruhlandt, R. H. Kehlenbach, F. S. Wouters, J. Grosshans, G. Bunt, J. Enderlein

Resolution doubling in fluorescence microscopy with confocal spinning-disk image scanning microscopy

Proceedings of the National Academy of Sciences of the United States of America 110 (52): p. 21000-21005 (2013)

6. F. Döring, A.L. Robisch, C. Eberl, M. Osterhoff, A. Ruhlandt, T. Liese, F. Schlenkrich, S. Hoffmann, M. Bartels, T. Salditt, H.U. Krebs

Sub-5 nm hard $x$-ray point focusing by a combined Kirkpatrick-Baez mirror and multilayer zone plate

Optics Express 21 (16): p. 19311-19323 (2013)

7. M. Osterhoff, M. Bartels, F. Döring, C. Eberl, T. Hoinkes, S. Hoffmann, T. Liese, V. Radisch, A. Rauschenbeutel, A.L. Robisch, A. Ruhlandt, F. Schlenkrich, T. Salditt, H.U. Krebs

Two-dimensional sub-5 $\mathrm{nm}$ hard x-ray focusing with MZP

Proceedings of SPIE $\mathbf{8 8 4 8}$ (2013)

8. A. Ruhlandt, T. Liese, V. Radisch, S. P. Kruger, M. Osterhoff, K. Giewekemeyer, H. U. Krebs, and T. Salditt

A combined Kirkpatrick-Baez mirror and multilayer lens for sub-10 nm x-ray focusing

AIP Advances 2: p. 012175 (2012) 


\section{Danksagung}

Diese Arbeit wäre nicht möglich gewesen ohne die kontinuierliche Unterstürzung vieler Menschen. An dieser Stelle möchte ich mich zuallererst bei Prof. Dr. Tim Salditt bedanken - nicht nur für die Ermöglichung dieser Arbeit am Institut für Röntgenphysik, sondern vor allem für fortlaufenden Zuspruch, Rat und viel entgegengebrachtes Vertrauen in den letzten sieben Jahren. Die Atmosphäre war stets durch große Freiheit geprägt, mit der Gelegeneit, vieles ausprobieren zu können und dabei ebenso viele, lehrreiche Fehler machen zu dürfen - an deren Ende durch guten Rat doch mancher Erfolg steht. Oft habe ich erst lange im Nachhinein bemerkt, wie wertvoll dabei manche Hinweise und Diskussionen waren.

Mein Dank gilt zudem Prof. Dr. Jörg Enderlein, der ohne zu zögern die Mitbetreuung dieser Arbeit übernommen und sie über vier Jahre begleitet hat. In diesem Zusammenhang möchte ich selbstverständlich auch Prof. Dr. Claus Ropers, Dr. Alexander Egner, Prof. Dr. Sarah Köster und Prof. Dr. Rainer Kree danken, die sich bereit erklärt haben, meinem Prüfungsausschuss anzugehören.

Unzählige Male habe ich mit Dr. Markus Osterhoff diskutiert, der immer ein offenes Ohr und fast genau so häufig einen guten Rat hatte - sei es bei Fragen zur Programmierung, technischen Details, physikalischen Grundlagen oder der Umgehung des Mensaessens. Keine Idee war zu verrückt, kein gewünschtes Fotomotiv war unmöglich. Mindestens genau so häufig habe ich mit Johannes Hagemann gerätselt, über kleine und große Probleme in schlecht konvergierenden Algorithmen, Untiefen der GPU Programmierung und der Welt im Allgemeinen. Bei beiden möchte ich mich hier für die vielen Einsichten, die konstruktive Kritik und ihre große Geduld bedanken.

Natürlich gilt auch meinen Bürokollegen ein besonderer Dank, insbesondere Martin Krenkel und Mareike Töpperwien. Wir haben nicht nur Stunden um Stunden in angenehmer, lustiger und produktiver Atmosphäre im Büro verbracht, sondern auch Konferenzen und Workshops besucht, uns die Nacht auf Messzeiten um die Ohren geschlagen, Carambar gegessen und über sehr viele Dinge diskutiert. Herzlichen Dank für diese schöne Zeit, die wertvolle Unterstützung bei Vorträgen, Veröffentlichungen und auch bei dieser Arbeit. Nicht ausgenommen werden darf an dieser Stelle natürich Jan-David Nicolas, der schon fast zum ,,Total Tollen Tomo-Team” gehört.

Überhaupt muss ich mich bei allen Mitarbeitern des Instituts für Röntgenphysik bedanken, für die außergewöhnlich gute Zusammenarbeit und die angenehme Arbeitsatmosphäre. Ich erinnere mich an zahllose leckere Kuchen, lustige Kaffeerunden (an denen ich gerne als Nicht-Kaffeetrinker teilgenommen habe), viele Ausflüge und manche Feier.

Mein ganz spezieller Dank gilt Jan Thiart, einem guten Freund, der mich nun schon seit dem zweiten Semester begleitet und der mit viel Einsatz versucht hat, die größten Rechtschreibfehler und sprachlichen Schnitzer aus dieser Arbeit zu vertreiben. Für alle, die übrig sind, trage ich allein die Verantwortung. 


\section{Danksagung}

Vor allen anderen muss ich an dieser Stelle aber meiner Familie danken: Meinen Eltern, deren kontinuierliche Unterstützung mein Studium überhaupt erst ermöglicht hat, allen, die zeitweise als Babysitter eingesprungen sind, und natürlich meiner lieben Frau Daja. Ohne ihre beständige Unterstützung und ihre grenzenlose Geduld wäre die Promotionszeit nicht halb so schön, bereichernd und vergnüglich gewesen. Insbesondere für ihren zuletzt unglaublichen Einsatz beim ,,Domptieren “ unserer beiden Kinder gebührt Daja mein allergrößter, herzlichster Dank. 


\section{Lebenslauf}

Name: $\quad$ Aike Ruhlandt

Geburtsdatum: 23. März 1988

Geburtsort: Eschwege

Nationalität: Deutsch

\section{Akademischer Werdegang:}

seit 02/2014 Wissenschaftlicher Mitarbeiter am Institut für

Röntgenphysik, Georg-August-Universität Göttingen

12/2013 Master of Science

Titel der Arbeit: „,Dreidimensionale Phasenrekonstruktion

in propagationsbasierter Phasenkontrast-Radiographie“

10/2011 - 12/2013 Studium der Physik, Georg-August-Universität Göttingen

09/2011 Bachelor of Science

Titel der Arbeit: „,Design und Berechnung eines kohärenten sub-20 nm Fokus für das Strahlrohr P10 an PETRA III“

10/2008 - 09/2011 Studium der Physik, Georg-August-Universität Göttingen

10/2007 - 06/2008 Zivildienst, Werraland-Werkstätten Eschwege

$06 / 2007$

Abitur, Oberstufengymnasium Eschwege 